Aus der Klinik für Neurologie

(Prof. Dr. med. M. Bähr)

der Medizinischen Fakultät der Universität Göttingen

\title{
Role of autophagic protein ULK1 in axonal degeneration and regeneration in rat cortical neurons in vitro
}

\author{
INAUGURAL-DISSERTATION \\ zur Erlangung des Doktorgrades \\ der Medizinischen Fakultät der \\ Georg-August-Universität zu Göttingen
}

vorgelegt von

Björn Friedhelm Vahsen

aus

Hildesheim

Göttingen 2021 
Dekan:

Referent/in:

Ko-Referent/in:

Drittreferent/in:
Prof. Dr. med. W. Brück

Prof. Dr. med. P. Lingor

Prof. Dr. rer. nat. M. Thumm

Prof. Dr. med. R. Dressel

Datum der mündlichen Prüfung: 02.11.2021 
Hiermit erkläre ich, die Dissertation mit dem Titel "Role of autophagic protein ULK1 in axonal degeneration and regeneration in cortical neurons in vitro" eigenständig angefertigt und keine anderen als die von mir angegebenen Quellen und Hilfsmittel verwendet zu haben.

Göttingen, den 10.04.2021

(Unterschrift) 
Die Daten, auf denen die vorliegende Arbeit basiert, wurden teilweise publiziert:

\section{Artikel in wissenschaftlichen Fachzeitschriften:}

Ribas VT*, Vahsen BF*, Tatenhorst L, Estrada V, Dambeck V, Almeida RA, Bähr M, Michel U, Koch JC, Müller HW, et al. (2021): AAV-mediated inhibition of ULK1 promotes axonal regeneration in the central nervous system in vitro and in vivo. Cell Death Dis 12,213

Vahsen BF, Lingor P (2021): ULK1 as a novel therapeutic target in neurodegeneration. Neural Regen Res $\underline{16}, 1212-1213$

Vahsen BF*, Ribas VT*, Sundermeyer J, Boecker A, Dambeck V, Lenz C, Shomroni O, Caldi Gomes L, Tatenhorst L, Barski E, et al. (2020): Inhibition of the autophagic protein ULK1 attenuates axonal degeneration in vitro and in vivo, enhances translation, and modulates splicing. Cell Death Differ $\underline{27}, 2810-2827$

Balke D*, Tatenhorst L*, Dambeck V, Ribas VT, Vahsen BF, Michel U, Bähr M, Lingor P (2020): AAV-mediated expression of dominant-negative ULK1 increases neuronal survival and enhances motor performance in the MPTP mouse model of Parkinson's disease. Mol Neurobiol $\underline{57}$, 685-697

*geteilte Erstautorenschaft

\section{Konferenzbeiträge:}

Vahsen BF, Ribas VT, Lenz C, Roser AE, Michel U, Urlaub H, Bähr M, Lingor P: Role of autophagic protein ULK1 in axonal degeneration and regeneration in cortical neurons in vitro. Poster im Rahmen des 11th FENS Forum of Neuroscience, Berlin, 07.07.-11.07.2018

Ribas VT, Vahsen BF, Lenz C, Michel U, Urlaub H, Bähr M, Lingor P: Role of autophagic protein ULK1 in axonal degeneration and regeneration after traumatic lesion to the central nervous system. Vortrag im Rahmen des 47th Annual Meeting of the Society for Neuroscience, Washington, DC, 11.11.-15.11.2017

Vahsen BF, Ribas VT, Lenz C, Michel U, Urlaub H, Bähr M, Lingor P: Role of ULK1 in axonal degeneration and regeneration in cortical neurons in vitro. Poster im Rahmen des 12th Göttingen Meeting of the German Neuroscience Society, Göttingen, 22.03.-25.03.2017

Ribas VT, Vahsen BF, Costa M, Michel U, Bähr M, Lingor P: Downregulation of autophagy attenuates axonal degeneration after traumatic lesion to the central nervous system. Poster im Rahmen des 10th Annual Canadian Neuroscience Meeting, Toronto, 29.05.-01.06.2016 


\section{Table of Contents}

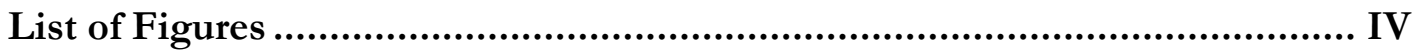

List of Tables ........................................................................................ VI

Abbreviations …..................................................................................... VII

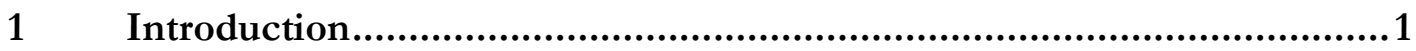

$1.1 \quad$ Neurodegeneration in the central nervous system ........................................................

1.2 Pathophysiology of axonal degeneration and regeneration after axonal injury ...............2

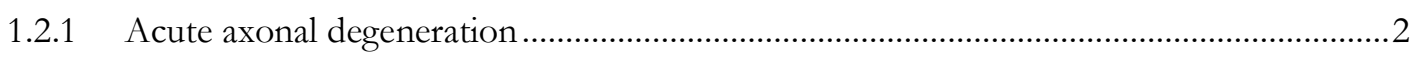

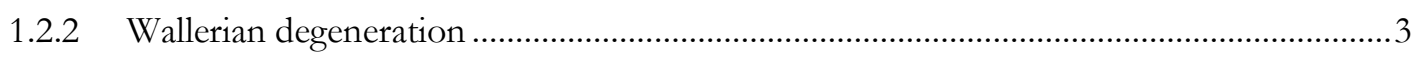

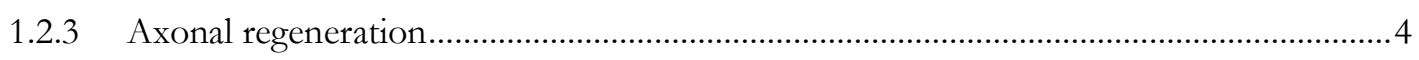

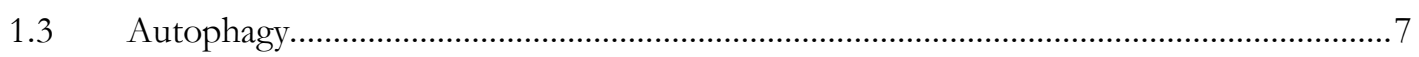

$1.4 \quad$ Uncoordinated 51-like kinase 1 (ULK1),...................................................................11

1.5 Autophagy and ULK1 in axonal degeneration and regeneration ...................................12

1.6 Methods to model axonal degeneration and regeneration after axonal injury in vitro ...14

1.6.1 Mechanical induction of axonal degeneration via scratch lesion assays ..........................14

1.6.2 Modeling axonal degeneration through pharmacological modulation of calcium

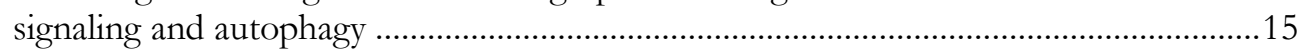

1.6.3 Selective axonal lesions in microfluidic culture platforms ............................................15

1.6.4 Mimicking the inhibitory, anti-regenerative environment after lesion.............................16

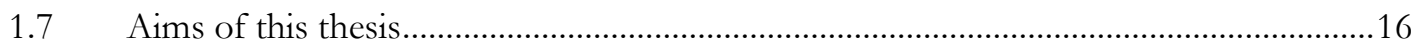

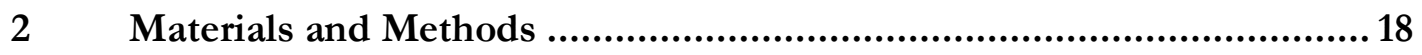

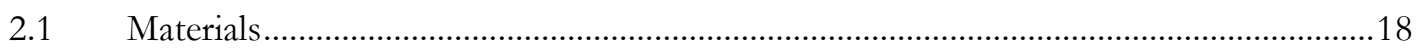

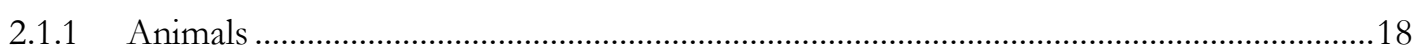

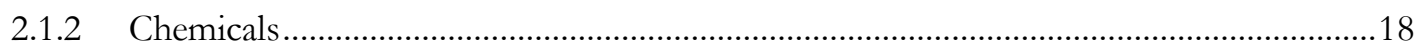

2.1.3 Buffers, solutions, and cell culture medium ...............................................................2

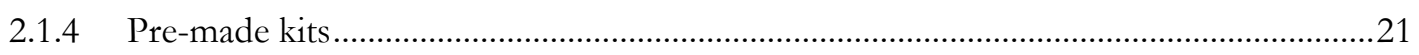

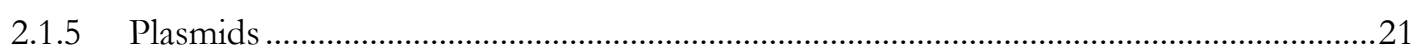

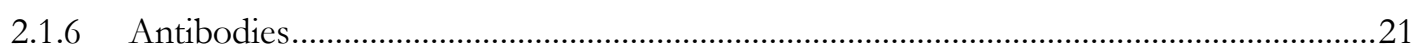

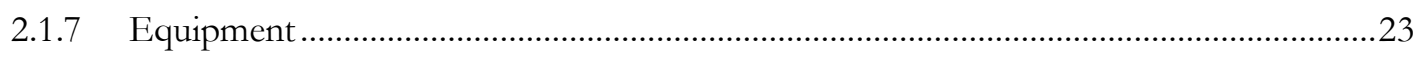

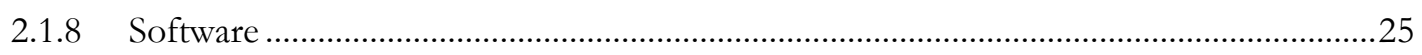

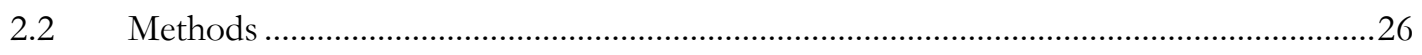

2.2.1 Cloning of plasmids and production of adeno-associated viral vectors ..........................26

2.2.2 Primary rat cortical neuron culture in culture plates ....................................................2.

2.2.3 Primary rat cortical neuron culture in microfluidic chambers .......................................... 31

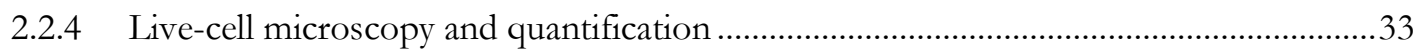

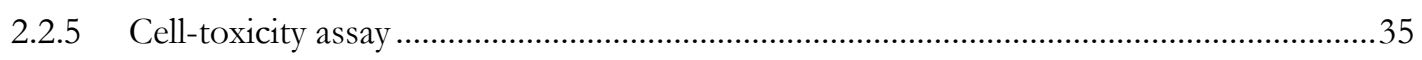

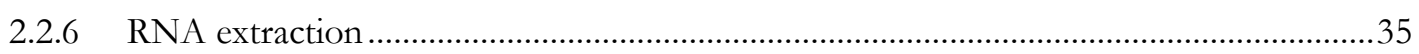

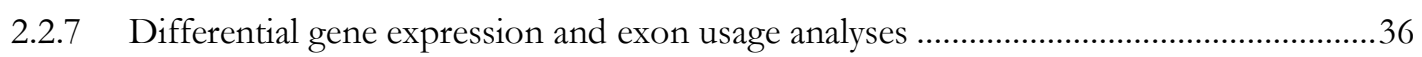

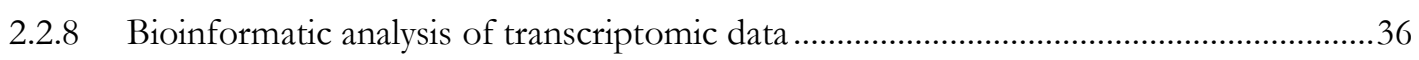

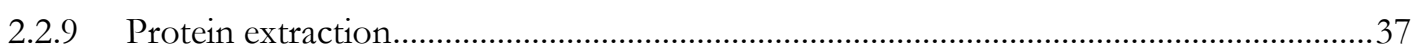




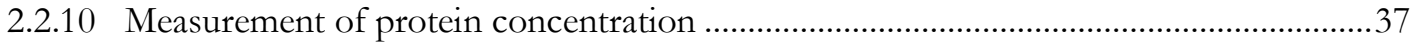

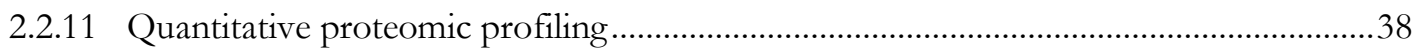

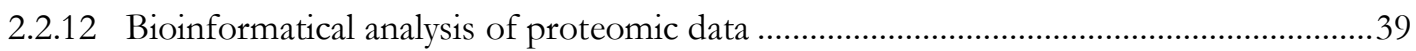

2.2.13 Sodium dodecyl sulfate-polyacrylamide gel electrophoresis ..........................................39

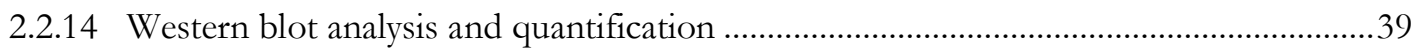

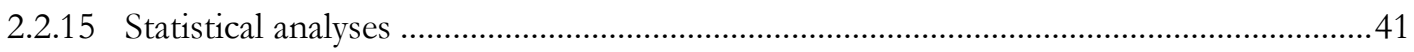

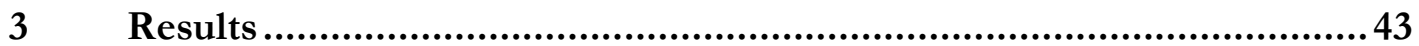

3.1 Adeno-associated viral vector-mediated overexpression of a dominant-negative form

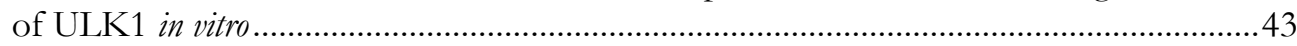

3.2 Evaluation of AAV vector toxicity................................................................................

3.3 Establishment of an in vitro model of autophagy induction and evaluation of the effect of AAV.ULK1.DN in this paradigm ......................................................................... 44

3.3.1 Assessment of scratch lesion-mediated autophagy induction ..........................................45

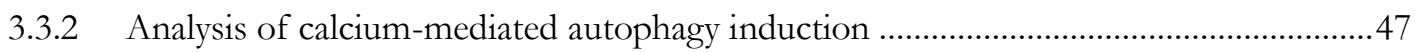

3.3.3 Evaluation of rapamycin-mediated autophagy induction ...............................................50

3.4 Transduction with AAV.ULK1.DN does not affect cell survival...................................55

3.5 AAV.ULK1.DN protects against acute axonal degeneration after axotomy in vitro .....56

3.6 Axonal regeneration after axotomy in vitro is enhanced by AAV.ULK1.DN ................58

3.7 AAV.ULK1.DN promotes neurite outgrowth on permissive and growth-inhibitory substrate in vitro

3.8 Molecular mechanisms of axon protection and regeneration - proteomic analysis of cortical neurons transduced with AAV.ULK1.DN ………………………………….......62

3.8.1 Proteomic changes induced by AAV.ULK1.DN ........................................................62

3.8.2 A prominent regulation of proteins associated with splicing and translation is mediated by AAV.ULK1.DN

3.9 Investigation of splicing - transcriptomic analysis of cortical neurons transduced with

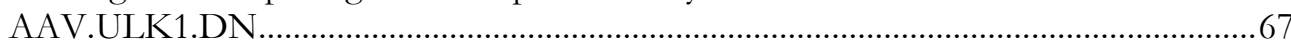

3.9.1 AAV.ULK1.DN results in differential splicing............................................................67

3.9.2 AAV.ULK1.DN modulates the splicing of axonal degeneration and regenerationassociated genes

3.10 AAV.ULK1.DN exerts neuroprotective and pro-regenerative effects by enhancing translation via an mTOR-S6-dependent mechanism......................................................71

3.11 AAV.ULK1.DN fosters axon growth and regeneration via increased ERK1 activity..76

3.12 AAV.ULK1.DN counteracts inhibitory environmental signaling after lesion via

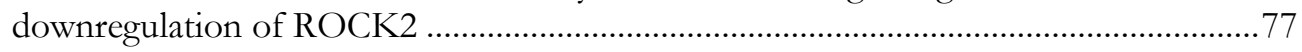

3.13 AAV.ULK1.DN does not enhance JAK-STAT3 signaling ............................................80

4 Discussion

4.1 Establishing an experimental paradigm to model autophagy induction after axonal

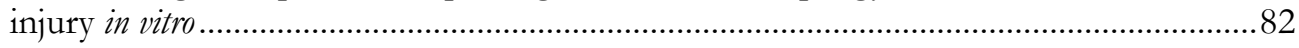

4.2 The effect of AAV.ULK1.DN on neuronal autophagy …………………….................. 84

4.3 Cell survival is not affected by AAV.ULK1.DN in vitro................................................. 84

4.4 AAV.ULK1.DN attenuates axonal degeneration in vitro................................................8

4.5 AAV.ULK1.DN exerts pro-regenerative effects in vitro..................................................86 
4.6 Understanding the molecular mechanisms of axon protection and axonal regeneration by AAV.ULK1.DN

4.6.1 Are the effects of AAV.ULK1.DN explained by autophagy inhibition? .......................87

4.6.2 AAV.ULK1.DN mediates the differential splicing of axon degeneration and regeneration-associated genes.

4.6.3 Differential splicing of Kif1 $b$ might mediate the beneficial effects of AAV.ULK1.DN via a non-canonical modulation of axonal transport

4.6.4 AAV.ULK1.DN leads to the differential splicing of Ddit3 and might protect from ER stress after axonal injury

4.6.5 Translation is enhanced by AAV.ULK1.DN via an mTOR-S6-dependent and PTENAKT-GSK3 $\beta$-independent mechanism....

4.6.6 AAV.ULK1.DN stimulates the intrinsic axonal growth capacity via increased ERK1 activation independent of JNK and STAT3 signaling.....

4.6.7 Inhibitory environmental signaling after lesion is counteracted by AAV.ULK1.DN via downregulation of ROCK2

4.7 AAV.ULK1.DN mediates axon protection and regeneration through a molecular switch from autophagy to axon-protective and growth-promoting pathways................95

4.8 Outlook and clinical relevance...................................................................................97

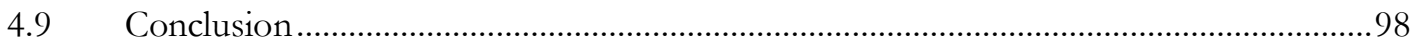

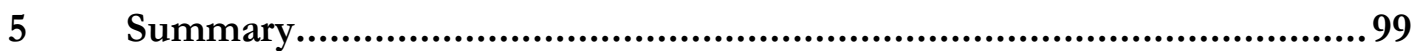

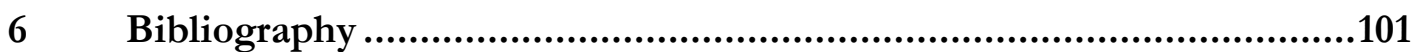




\section{List of Figures}

Figure 1: Overview of the morphological and molecular events during acute axonal

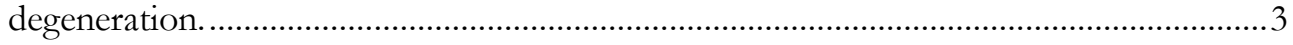

Figure 2: Overview of the signaling pathways involved in axonal growth and regeneration. ......5

Figure 3: Schematic overview of mammalian ULK1-dependent autophagy. ............................ 10

Figure 4: Detailed structure and phosphorylation sites of ULK1 ..........................................11

Figure 5: The involvement of autophagic proteins in axonal degeneration after axonal injury. 13

Figure 6: Overview of the microfluidic culture system........................................................15

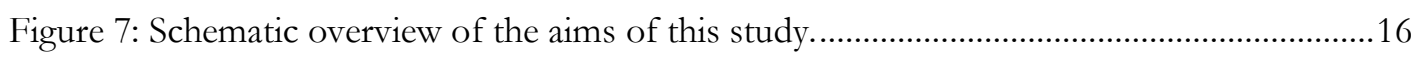

Figure 8: Adeno-associated viral vectors used in this thesis.....................................................26

Figure 9: AAV.ULK1.DN-mediated overexpression of dominant-negative ULK1 in rat cortical neurons in vitro.

Figure 10: ToxiLight ${ }^{\mathrm{TM}}$ assay of cytotoxicity after transduction with different titers of AAV.mCherry and AAV.ULK1.DN.

Figure 11: Autophagy markers after scratch lesion on DIV 3/4 and transduction with AAV.ULK1.DN.

Figure 12: Autophagy markers after scratch lesion on DIV 7 and transduction with AAV.ULK1.DN.

Figure 13: Autophagy markers after addition of calcium ionophore on DIV 4 and transduction with AAV.ULK1.DN.

Figure 14: Autophagy markers after addition of calcium ionophore on DIV 7 and transduction with AAV.ULK1.DN.

Figure 15: Autophagy induction after addition of rapamycin on DIV 4 is mildly inhibited after transduction with AAV.ULK1.DN.

Figure 16: Autophagy induction after addition of rapamycin on DIV 7 is inhibited after transduction with AAV.ULK1.DN.

Figure 17: ULK1, ATG5, and ATG7 levels after rapamycin treatment on DIV 7 and transduction with AAV.ULK1.DN.....

Figure 18: Cleaved caspase 3 levels remain unchanged after rapamycin treatment and transduction with AAV.ULK1.DN.

Figure 19: Evaluation of cell survival after staurosporine-mediated apoptosis induction and transduction with AAV.ULK1.DN.....

Figure 20: AAV.ULK1.DN attenuates AAD after selective axonal lesion in vitro. ....................57

Figure 21: AAV.ULK1.DN fosters axonal regeneration after axotomy in vitro...........................59

Figure 22: AAV.ULK1.DN enhances neurite outgrowth of rat cortical neurons cultured on permissive and non-permissive substrate in vitro.....

Figure 23: Quantitative proteomic profiling reveals 122 significantly regulated proteins after transduction with AAV.ULK1.DN.

Figure 24: Overview of proteins with strongest up- and downregulation in quantitative proteomic profiling after transduction with AAV.ULK1.DN and Western blot validation of two regulated targets.

Figure 25: Overview of interactions between proteins with significant regulation in proteomic profiling after transduction with AAV.ULK1.DN.

Figure 26: Differential exon expression analysis reveals AAV.ULK1.DN modulates differential splicing.

Figure 27: AAV.ULK1.DN mediates differential splicing of genes involved in axonal degeneration and regeneration.

Figure 28: AAV.ULK1.DN enhances mTOR signaling. ............................................................ 72

Figure 29: AAV.ULK1.DN reduces the levels of p-AMPK......................................................73 
Figure 30: AAV.ULK1.DN moderately reduces p-AKT levels...................................................74

Figure 31: PTEN levels are not altered by AAV.ULK1.DN. .....................................................

Figure 32: GSK3 $\beta$ expression is not altered by transduction with AAV.ULK1.DN...................75

Figure 33: AAV.ULK1.DN increases the levels of p-ERK1 ……………................................76

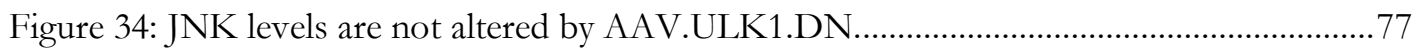

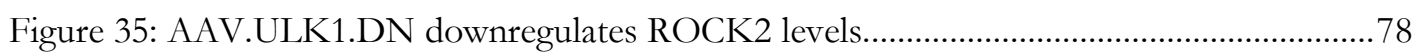

Figure 36: CRMP2 levels are not altered by AAV.ULK1.DN ………………….......................78

Figure 37: AAV.ULK1.DN moderately reduces p-STAT3 levels. ………………….................. 80

Figure 38: Summary of the findings on the role of ULK1 in axonal degeneration and

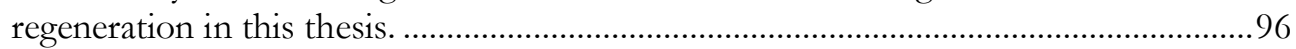




\section{List of Tables}

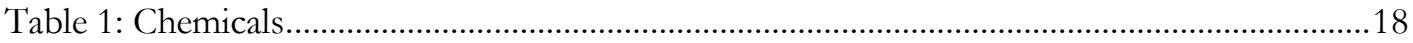

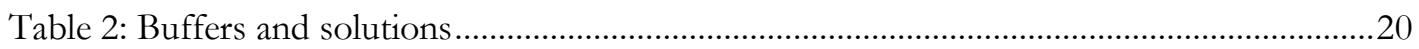

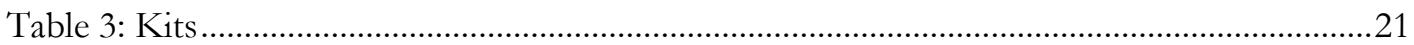

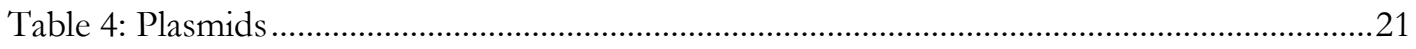

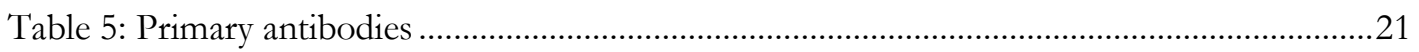

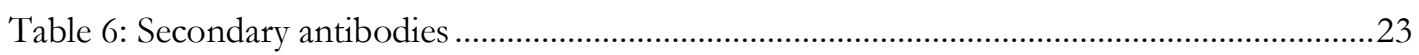

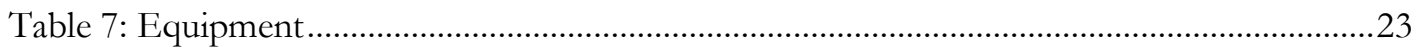

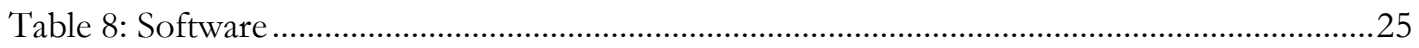

Table 9: Cell numbers in different cell culture experiments ......................................................28

Table 10: Virus titers employed in different experimental paradigms .....................................29

Table 11: Antibodies and dilutions used for Western blotting .....................................................40

Table 12: Biological processes annotated to proteins with significant regulation in quantitative proteomic profiling after transduction with AAV.ULK1.DN ....................................64

Table 13: Cellular components annotated to proteins with significant regulation in quantitative proteomic profiling after transduction with AAV.ULK1.DN.

Table 14: Biological processes annotated to genes with significantly different exon usage after transduction with AAV.ULK1.DN

Table 15: Molecular functions annotated to genes with significantly different exon usage after transduction with AAV.ULK1.DN

Table 16: Cellular components annotated to genes with significantly different exon usage after transduction with AAV.ULK1.DN 


\section{Abbreviations}

3-MA

4E-BP1

a.u.

AAD

AAV

ADP

AK

ALS

AMP

AMPK $\alpha$

ANOVA

APS

ATG

ATP

BAF

BCA

BSA

CC

cDNA

CMF

CNS

CRMP2

CSPG

CTD

CTR

DAVID

Ddit3

Dept.

DIV

DNA

DNase

DOP

DTT

E18

ECL

EDTA

ER

ERK

$\mathrm{fc}$ 3-methyladenine

eukaryotic translation initiation factor 4E-binding protein 1

arbitrary units

acute axonal degeneration

adeno-associated viral

adenosine diphosphate

adenylate kinase

amyotrophic lateral sclerosis

adenosine monophosphate

AMP-activated protein kinase alpha

analysis of variance

ammonium persulfate

autophagy-related protein

adenosine triphosphate

bafilomycin

bicinchoninic acid

bovine serum albumin

Creative Commons

complementary DNA

calcium magnesium-free

central nervous system

collapsin response mediator protein 2

chondroitin sulfate proteoglycan

C-terminal domain

control

Database for Annotation, Visualization and Integrated Discovery

DNA damage inducible transcript 3

Department

day in vitro

deoxyribonucleic acid

deoxyribonuclease

day of preparation

dithiothreitol

embryonic day 18

enhanced chemiluminescence

ethylenediaminetetraacetic acid

endoplasmic reticulum

extracellular signal-regulated kinase

fold change 


\begin{tabular}{|c|c|}
\hline FCS & fetal calf serum \\
\hline FDR & false discovery rate \\
\hline FIP200 & focal adhesion kinase family-interacting protein of $200 \mathrm{kDa}$ \\
\hline GAPDH & glyceraldehyde 3-phosphate dehydrogenase \\
\hline $\mathrm{GO}$ & gene ontology \\
\hline GSK3 $\beta$ & glycogen synthase kinase 3 beta \\
\hline GTP & guanosine triphosphate \\
\hline HBSS & Hank's balanced salt solution \\
\hline HEPES & 4-(2-hydroxyethyl)-1-piperazineethanesulfonic acid \\
\hline HRP & horseradish peroxidase \\
\hline hSyn & human synapsin \\
\hline iPSC & induced pluripotent stem cell \\
\hline JAK & Janus kinase \\
\hline JNK & c-Jun N-terminal kinase \\
\hline Kif1b & kinesin family member $1 \mathrm{~b}$ \\
\hline $\mathrm{LC} 3$ & microtubule-associated protein 1 light chain 3 \\
\hline LIMK1 & LIM domain kinase 1 \\
\hline LIR & LC3-interacting \\
\hline MAPK & mitogen-activated protein kinase \\
\hline Mapkap3 & MAPK activated protein kinase 3 \\
\hline MAPKK & MAPK kinase \\
\hline MC & medium change \\
\hline mTOR & mammalian target of rapamycin \\
\hline mTORC1 & mTOR complex 1 \\
\hline N.S. & no significant difference \\
\hline NAD & nicotinamide adenine dinucleotide \\
\hline nanoLC-MS/MS & nanoscale liquid chromatography coupled to tandem mass spectrometry \\
\hline NMN & nicotinamide mononucleotide \\
\hline NMNAT2 & NMN adenylyltransferase 2 \\
\hline $\mathrm{ON}$ & overnight \\
\hline $\mathrm{ONC}$ & optic nerve crush \\
\hline p- & phospho- \\
\hline PAK2 & p21-activated kinase 2 \\
\hline PBS & phosphate-buffered saline \\
\hline PD & Parkinson's disease \\
\hline PDL & poly-D-lysine \\
\hline $\mathrm{PE}$ & phosphatidylethanolamine \\
\hline PI3K & phosphatidylinositol 3-kinase \\
\hline PI3P & phosphatidylinositol 3-phosphate \\
\hline PLO & poly-L-ornithine \\
\hline PNS & peripheral nervous system \\
\hline
\end{tabular}


PSN penicillin/streptomycin/neomycin

PTEN phosphatase and tensin homolog

Ptprf protein tyrosine phosphatase receptor type $\mathrm{f}$

PVDF polyvinylidene difluoride

RAP rapamycin

RM repeated measurement

RNA ribonucleic acid

ROCK2 Rho-associated kinase 2

RT

$\mathrm{S} / \mathrm{P}$

room temperature

S6

serine- and proline-rich

SCI

S6 ribosomal protein

SDS spinal cord injury

SDS-PAGE sodium dodecyl sulfate

siRNA SDS-polyacrylamide gel electrophoresis

SMN small interfering RNA

SQSTM1

survival motor neuron

STA

STAT3 sequestosome 1

STRING staurosporine

STRING signal transducer and activator of transcription 3

SWATH sequential window acquisition of all theoretical fragment-ion spectra

TAOK1 thousand and one amino acid protein kinase 1

TBS Tris-buffered saline

TBS-T TBS-Tween-20

TDP-43 transactive response DNA-binding protein of $43 \mathrm{kDa}$

TEMED tetramethylethylenediamine

TU transducing unit

UBA ubiquitin-associated

ULK1 uncoordinated 51-like kinase 1

ULK1.DN dominant-negative ULK1

VPS vacuolar protein sorting

Wallerian degeneration 


\section{Introduction}

\subsection{Neurodegeneration in the central nervous system}

In several traumatic and degenerative disorders of the central nervous system (CNS), such as spinal cord injury (SCI) and amyotrophic lateral sclerosis (ALS), neuronal demise is a key pathological feature and major cause of progressive clinical disability. To combat neuronal loss in the course of these deleterious diseases, early therapeutic endeavors aimed to protect the neuronal cell body from degeneration but proved unsuccessful in long-term trials and human patients. In fact, it is now becoming increasingly clear that in both traumatic and neurodegenerative diseases, degeneration of the axon - rather than the soma - represents the early and initial pathological mechanism and often precedes neuronal death (Coleman 2005; Fischer et al. 2004; Lingor et al. 2012). Furthermore, while axonal regeneration is routinely observed in the peripheral nervous system (PNS), the regenerative capacity of axons in the adult CNS is very limited (Liu et al. 2011). A better understanding of the mechanisms of axonal degeneration and regeneration in the CNS is therefore hoped to unravel new therapeutic avenues that either protect axons against degeneration or enhance their ability to regenerate, ultimately making neuroprotective therapies possible (Lingor et al. 2012; Medana and Esiri 2003).

Axonal degeneration can be reproducibly induced using experimental paradigms of traumatic axonal injury; hence, many publications in the field have focused on the mechanisms of degeneration after axonal lesion. As a result, it has now emerged that, while initially considered a rather unspecific process, axonal degeneration should be comprehended as a finely regulated cascade of biochemical events executing a delicate mechanism of selfdestruction that is different from programmed cell death. This increased understanding of the process and the growing body of evidence have opened up the possibility of targeted experimental interference in recent years. It is hoped that the modulation of one or several key molecules will demonstrate sufficient therapeutic potential to counteract lesion-induced axon loss. Additionally, it is likely that promising findings can be translated into the potential treatment of neurodegenerative disorders, as there is considerable overlap in the pathophysiological cascade underlying degenerative and traumatic CNS disorders, albeit diverging etiologies (Lingor et al. 2012; Raff et al. 2002). 


\subsection{Pathophysiology of axonal degeneration and regeneration after axonal injury}

Landmark studies (Kerschensteiner et al. 2005; Knoferle et al. 2010) on traumatic axonal injury in the CNS have elucidated the pathophysiology of axonal degeneration, including its temporal and spatial kinetics. Post-injury axonal degeneration can therefore be subdivided into two morphologically different phases: an acute phase, called acute axonal degeneration (AAD), which rapidly occurs on both axonal sides of the lesion, and a sub-acute process involving slow dieback degeneration in the proximal axon remnant and degeneration of the distal axonal part termed Wallerian degeneration (WD) (Waller 1850). WD might then be followed by or overlap with a third phase of regenerative attempts in the proximal remnant of the axon, influenced and restricted by intrinsic axonal factors and extrinsic modulation through glial cells (Lingor et al. 2012; Wang et al. 2012)

\subsubsection{Acute axonal degeneration}

Experiments on the spinal cord and optic nerve demonstrated that, within several hours after a focal traumatic axonal lesion, the axonal parts on both sides of the injury undergo AAD, which is characterized by progressive fragmentation spanning up to $400 \mu \mathrm{m}$ in length (Kerschensteiner et al. 2005; Knoferle et al. 2010).

During AAD, for ten to thirty minutes after lesion, axons remain morphologically unchanged on a microscopic level. However, on a molecular level, rapid influx of calcium is initiated almost immediately after axonal injury, resulting in increased intra-axonal calcium concentrations within as short as 40 seconds (Figure 1). As a proof of concept, pharmacological inhibition of calcium channels blocks AAD almost completely, while increased calcium influx following application of the calcium ionophore A23187 significantly aggravates AAD (Knoferle et al. 2010; Ribas and Lingor 2016; Ribas et al. 2017). Consequently, calcium-dependent proteases such as calpain are activated (Figure 1). Calpain induces the activation and modulation of a plethora of molecular substrates, including cleavage of the collapsin response mediator protein 2 (CRMP2) (Zhang et al. 2016). Calpain inhibition or CRMP2 overexpression were both shown to attenuate AAD after optic nerve crush (ONC) or SCI (Kerschensteiner et al. 2005; Zhang and Koch 2017; Zhang et al. 2016).

Within 30 to 120 minutes after lesion, this leads to the condensation and misalignment of neurofilaments and subsequent fragmentation of microtubules (Figure 1). This disintegration of the cytoskeleton, likely in combination with dysfunctional axonal transport, results in the formation of local axonal swellings (termed bulbs), which also become visible on a 
microscopic level (Figure 1) (Coleman 2005; Wang et al. 2012). Within these bulbs, organelles, such as mitochondria and autophagosomes, accumulate (Lingor et al. 2012). It is assumed that this accumulation of autophagosomes is a reflection of detrimental autophagy induction, as both pharmacological inhibition of calcium channels and blockage of autophagy using 3-methyladenine (3-MA) lead to a reduction in autophagy and attenuation of AAD (Knoferle et al. 2010), indicating that autophagy activation post-injury represents an important molecular step in the execution of AAD and potential therapeutic target.

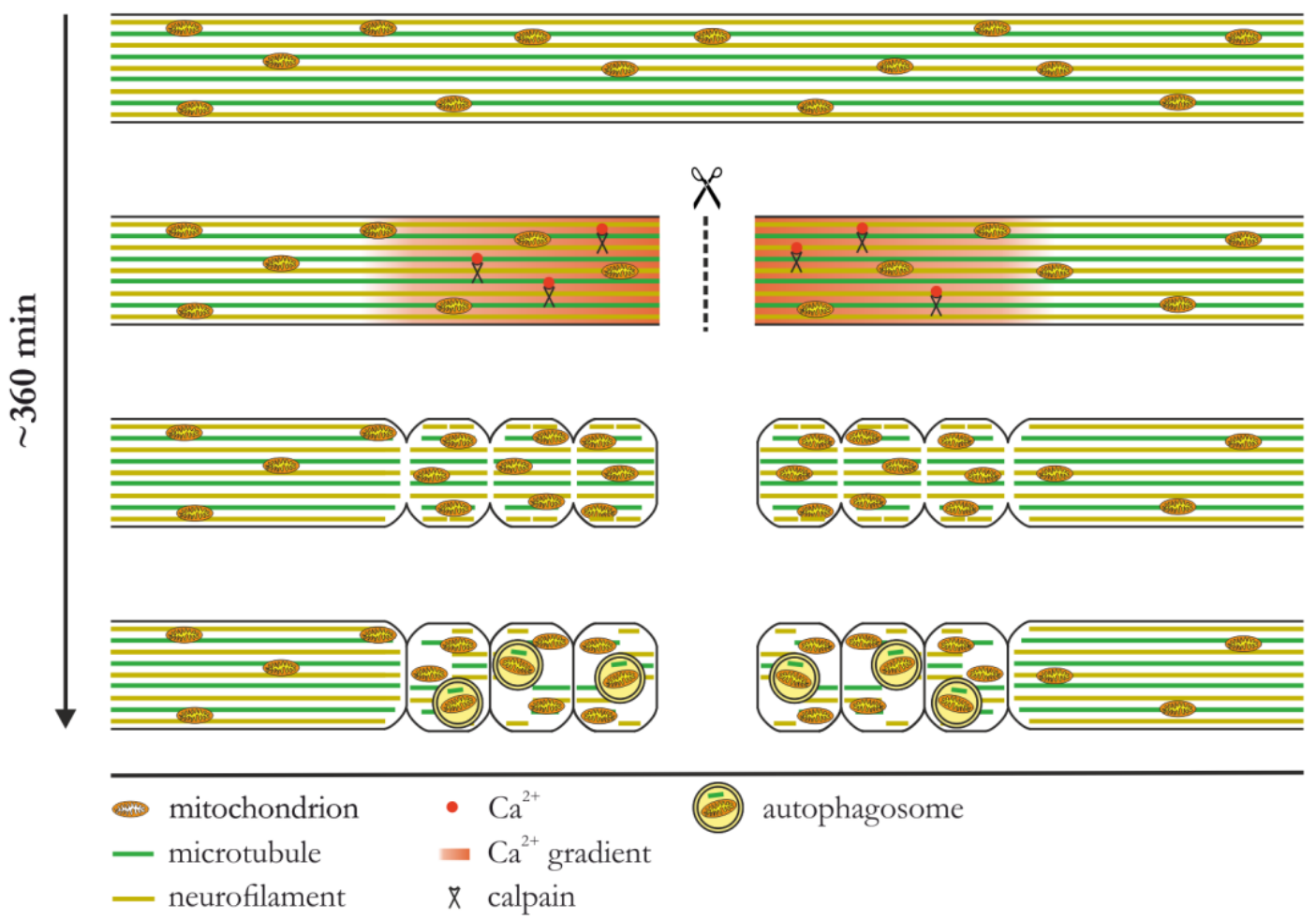

Figure 1: Overview of the morphological and molecular events during acute axonal degeneration. A traumatic lesion to an axon in the CNS results in rapidly increased intracellular calcium levels (within $30-40$ s). Activation of calcium-dependent enzymes, such as calpain, leads to the disruption of microtubules and misalignment of neurofilaments. As a consequence, impaired axonal transport, for instance of mitochondria, results in the local accumulation of organelles and subsequent formation of axonal bulbs (within $30-120 \mathrm{~min}$ ). In the following, autophagosomes are formed in order to clear away cytoskeletal fragments and organelles, which is accompanied by axonal fragmentation on a microscopic level. Figure adapted from Lingor et al. (2012), licensed under a Creative Commons (CC) BY 2.0 license, https://creativecommons.org/licenses/by/2.0/.

\subsubsection{Wallerian degeneration}

For 24 to $72 \mathrm{~h}$ after axonal injury, the distal part of a transected axon remains morphologically unchanged and is still electrically excitable (Wang et al. 2012). Hereafter, progressive fragmentation, termed WD, is initiated and ultimately leads to the complete removal of the distal fragment of the axon (Conforti et al. 2014; Waller 1850). The molecular mechanisms underlying WD are not yet entirely understood. Currently, it is suggested that a 
key mechanism in WD after lesion lies in the impaired delivery of the essential survival factor nicotinamide mononucleotide adenylyltransferase 2 (NMNAT2) from the soma to the distal parts of the axon (Coleman and Hoke 2020). Mechanistically, loss of NMNAT2 reduces the levels of the coenzyme nicotinamide adenine dinucleotide (NAD), while increasing the levels of its precursor nicotinamide mononucleotide (NMN) (Hill et al. 2016). This rise in NMN, NAD depletion or a combination of both is thought to ultimately trigger WD (Di Stefano et al. 2015; Gerdts et al. 2015). The potential downstream effector mechanism of the NMNAT2 pathway is assumed to be the sterile alpha and TIR motif-containing 1 (SARM1) protein, which is a mediator of rapid axonal fragmentation (Gerdts et al. 2015; Gilley et al. 2015; Osterloh et al. 2012). Physical fragmentation of the axon attracts glial cells, mainly astrocytes and macrophages, which then clear axonal debris and modulate regeneration of the proximal axon stump (Wang et al. 2012).

\subsubsection{Axonal regeneration}

Regenerative attempts might be seen as early as 24 hours post-injury in adult CNS axons in vivo (Kerschensteiner et al. 2005). Similar to axon growth during neurodevelopment, a critical requirement for axon regeneration after lesion is the formation of a growth cone, in which cytoskeletal proteins are arranged and connected. For this, expression of growth-associated genes, translation of relevant proteins, and then dynamic formation of actin and microtubules are key requirements (Curcio and Bradke 2018). However, axons in the adult CNS usually do not form new extensions beyond the point of the original injury, as axon regeneration is inhibited by a combination of low intrinsic growth potential and a non-permissive extrinsic environment after lesion consisting of soluble and glia cell-bound inhibitory molecules (Fawcett 2020).

After lesion, inhibitory molecules bound to the membrane of oligodendrocytes, such as Nogo proteins, myelin-associated glycoprotein, or oligodendrocyte-myelin glycoprotein, exert growth-inhibiting properties through activation of the trimeric Nogo receptor $(\mathrm{NgR}) /$ p75 neurotrophin receptor $\left(\mathrm{p} 75^{\mathrm{NTR}}\right) /$ leucine-rich repeat Ig domain-containing Nogointeracting protein 1 (LINGO1) receptor complex (Lingor et al. 2007). Upon stimulation, the receptor complex activates the small guanosine triphosphate (GTP)ase RhoA (Figure 2). RhoA stimulates the Rho-associated kinase 2 (ROCK2), which then triggers LIM domain kinase 1 (LIMK1). LIMK1 leads to the phosphorylation-mediated inactivation of cofilin, resulting in inhibited actin dynamics and thus impaired axonal growth. This effect is further amplified by a direct inhibitory effect of ROCK2 on CRMP2, leading to reduced microtubule assembly (Koch et al. 2014b). Similarly, chondroitin sulphate proteoglycans (CSPGs), 
produced by astrocytes and oligodendrocytes after lesion, stimulate the RhoA-ROCK-LIMK pathway, amplifying the anti-regenerative effects after lesion (Niederost et al. 1999). Both downregulation of RhoA and inhibition of ROCK2 led to increased neurite outgrowth and axon regeneration in different lesion models, enabling at least partial antagonism of the nonpermissive environmental signals after lesion (Challagundla et al. 2015; Koch et al. 2014a; Koch et al. 2014b; Lingor et al. 2007).

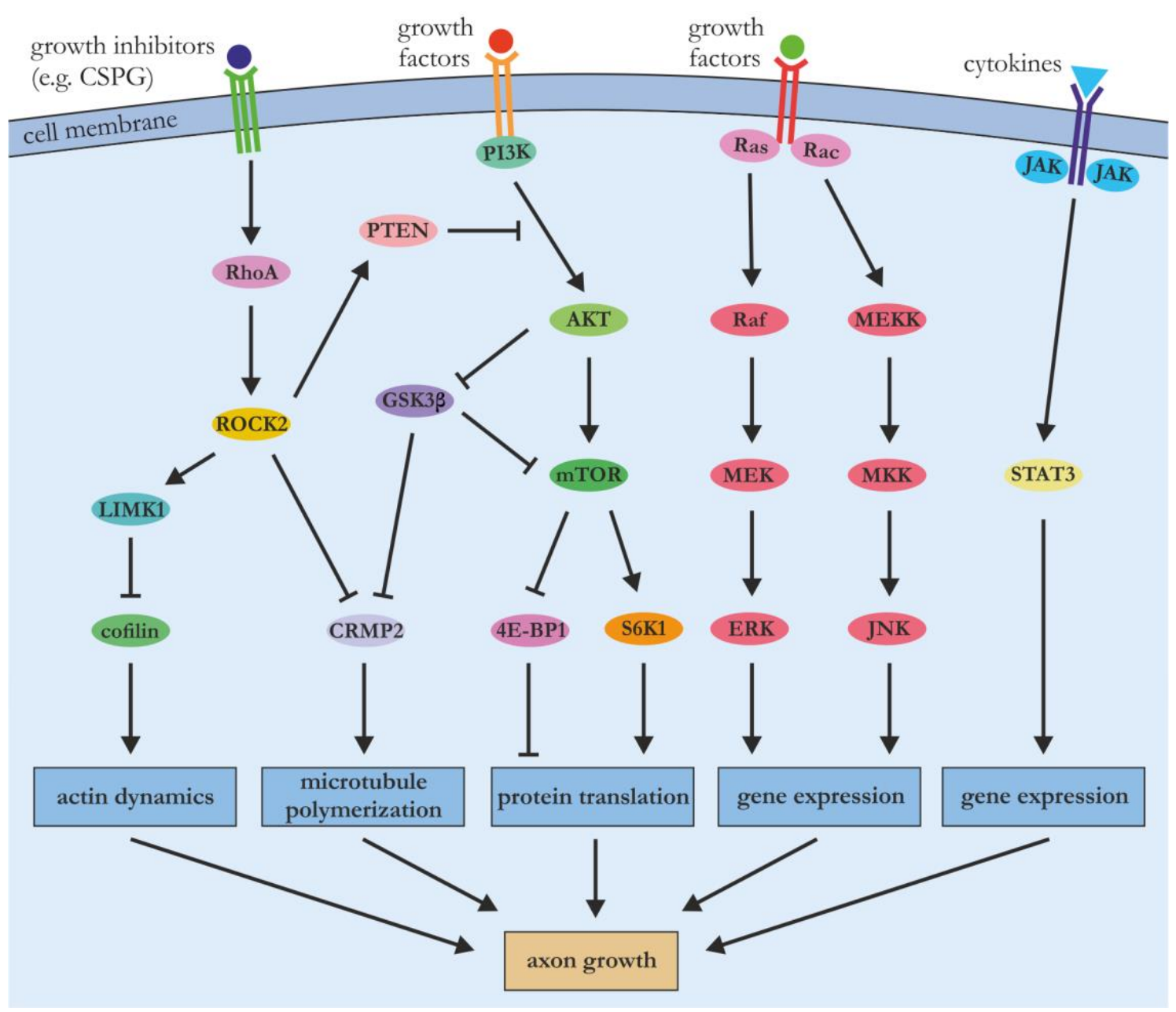

Figure 2: Overview of the signaling pathways involved in axonal growth and regeneration. After axonal lesion, growth-inhibiting molecules such as CSPGs bind to their respective membrane receptors, activating RhoA-ROCK2 signaling. This results in the inhibition of actin dynamics and microtubule polymerization, leading to impaired axon outgrowth. On the other hand, axon growth is endogenously controlled via stimulation of different receptors through various growth factors and cytokines. Activation of these receptors leads to increased protein translation via instigation of the PI3K-AKT-mTOR pathway, or modulation of gene expression through the MAPK pathway (e.g. Ras-Raf-MEK-ERK) or JAK-STAT3 signaling. Many of the proteins involved in these growth-enhancing pathways are upregulated during neurodevelopment but show decreased expression in adult CNS neurons. Potential therapeutic approaches for pro-regenerative therapies therefore aim to either dampen the activation of RhoA-ROCK2 signaling or enhance growth-promoting pathways after axonal injury. Figure adapted from Barros Ribeiro da Silva et al. (2020), with kind permission from American Chemical Society. 
Additionally, axonal regeneration is controlled via various endogenous pathways (Figure 2), the importance of which for axon growth was demonstrated in neurodevelopmental studies and experiments on the regeneration of PNS axons (Fawcett 2020). These pathways regulate gene expression in the soma and cytoskeletal dynamics in the growth cone but are hampered in injured adult neurons. Many of the proteins involved converge on the regulation of the growth master controller mammalian target of rapamycin (mTOR). mTOR is a serine/threonine protein kinase that activates the production of ribosomes and induces translation via activation of its downstream targets S6 kinase 1 (S6K1) as well as S6 ribosomal protein (S6) and inhibition of eukaryotic translation initiation factor 4E-binding protein 1 (4E-BP1), respectively (Curcio and Bradke 2018). A boost in translation is critical for the biosynthesis of new cytoskeletal proteins and other growth-associated factors during axon regeneration. However, mTOR activity declines in the adult cell and following axonal injury, leading to a significant impairment of axon regeneration in the CNS (Liu et al. 2011).

A major regulator of mTOR activity is the phosphatase and tensin homolog (PTEN)phosphatidylinositol 3-kinase (PI3K)-AKT pathway (Figure 2). In this pathway, stimulation of membrane-bound tyrosine kinases by growth factors, for instance nerve growth factor (NGF), results in the activation of PI3K, leading to AKT-mediated mTOR stimulation. However, the growth-inhibitory RhoA-ROCK2 pathway activates PTEN, which inhibits PI3K activity, resulting in reduced mTOR activity and impaired growth. Experimental deletion of PTEN was demonstrated to result in robust axon regeneration via increased mTOR-dependent translation (Park et al. 2008). Similarly, overexpression of active AKT resulted in increased axonal regeneration (Namikawa et al. 2000). This effect is not only explained via directly increased mTOR activity but also through regulation of another downstream target of AKT: glycogen synthase kinase 3 beta (GSK3 $\beta$ ). GSK3 $\beta$ inhibits mTOR but also mediates inhibition of CRMP2 function through phosphorylation, thereby amplifying RhoA-ROCK2 signaling and resulting in impaired growth cone dynamics (Figure 2). In contrast, deletion of GSK3 $\beta$ or overexpression of CRMP2 lead to increased axon regeneration (Curcio and Bradke 2018; Leibinger et al. 2019; Leibinger et al. 2017). Taken together, modulation of the PTEN-PI3K-AKT pathway as well as the interconnected GSK3 $\beta$-CRMP2 axis is therefore a promising tool to foster axonal regeneration.

Another pathway of crucial relevance for axonal growth and regeneration is the mitogenactivated protein kinase (MAPK)/ extracellular signal-regulated kinase (ERK) pathway (Figure 2). Binding of growth factors to receptor-linked tyrosine kinases and other receptors results in a cascade of biochemical phosphorylation events that leads to the activation of 
downstream targets: Receptor activation triggers the activation of a member of the Ras family of small GTP-binding proteins. Ras then activates a series of kinases, firstly MAP kinase kinase kinase (MAPKKK)/ Raf, which activates MAP kinase kinase (MAPKK)/ MEK1/2 leading to the activation of MAPK/ ERK1/2 (Cargnello and Roux 2011). Activated ERK1/2 regulate a vast array of transcription factors involved in cellular growth. Specifically, ERK1/2 were shown to be required for the formation of a new growth cone and axon elongation (Chierzi et al. 2005; Raivich and Makwana 2007). Similarly, another member of the MAPK family, c-Jun N-terminal kinase (JNK), which is activated by a comparable biochemical cascade via Rac-MEKK-MKK (Figure 2), was implicated in axonal growth. For instance, absence of the JNK target c-Jun severely impaired the expression of regeneration-associated genes after axonal injury (Raivich et al. 2004). Modulation of the MAPK pathway is therefore also of great relevance for axonal regeneration.

Finally, cytokines, such as ciliary neurotrophic factor (CNTF), were shown to bind to another class of receptors named Janus kinase (JAK) receptors (Figure 2). Activation of JAK receptors leads to phosphorylation-induced activation of different signal transducers and activators of transcription (STATs), very importantly STAT3. Overexpression or hyperactivation of STAT3 increased axon regeneration via expression of pro-regenerative genes (Barros Ribeiro da Silva et al. 2020; Curcio and Bradke 2018), rendering the JAKSTAT3 pathway another key target to promote regeneration after axonal injury.

Taken together, potential therapeutic approaches for pro-regenerative therapies should therefore aim to inhibit activation of RhoA-ROCK2 signaling or enhance one or several of the growth-promoting pathways after axonal injury (Figure 2). Intriguingly, many of these signaling cascades are closely connected to the cellular homeostatic process autophagy.

\subsection{Autophagy}

The nature of neurons as highly metabolically active, long-lived, and usually postmitotic cells requires efficient systems for the removal of aged or dysfunctional organelles and damaged or aggregated proteins (Stavoe and Holzbaur 2019). Multiple different pathways for degradation in neurons have been described, including the ubiquitin-proteasome pathway and lysosome-autophagy (from Greek, self-eating) systems, such as chaperone-mediated autophagy, microautophagy, and macroautophagy. The ubiquitin-proteasome pathway clears abnormally folded or short-lived proteins via ubiquitylation and subsequent proteasomal breakdown (Kleiger and Mayor 2014). Chaperone-mediated autophagy employs chaperone proteins for the selective delivery of proteins to the lysosome for degradation (Cuervo and 
Wong 2014), while microautophagy enables the engulfment of cytoplasmic cargo via invagination through the lysosomal membrane (Li et al. 2012). Macroautophagy, however, which is considered the major autophagic mechanism, forms double-membraned structures, named autophagosomes, around cargo and delivers it to the lysosome (Menzies et al. 2017). The following parts of this thesis will focus on macroautophagy, which will thus henceforth be referred to as autophagy.

Autophagy is responsible for the turnover of long-lived organelles, proteins and other cytosolic components, enabling the digestion and recycling of nutrients (Menzies et al. 2015). Basal and constitutive levels of autophagy are therefore required to maintain neuronal homeostasis in the CNS (Nikoletopoulou et al. 2015). Mechanistically, the autophagy cascade is characterized by multiple interrelated steps that include initiation, elongation, completion, and degradation (Figure 3) (Rubinsztein et al. 2015). Upon activation of autophagy, a firstly single- then double-membraned precursor of cup-like shape (termed phagophore) is formed, which is capable of engulfing cargo. This phagophore progressively extends to ultimately form a closed autophagosome, which then fuses with endosomes and/or lysosomes to enable degradation of the contents and finally release of the breakdown products (Menzies et al. 2017; Yang and Klionsky 2010).

In response to low energy levels, growth factor deprivation, and nutrient depletion, autophagy is initiated via signaling pathways that lead to the activation of the uncoordinated 51-like kinase 1 (ULK1) complex (Figure 3). The ULK1 complex is comprised of ULK1, focal adhesion kinase family-interacting protein of $200 \mathrm{kDa}$ (FIP200), autophagy-related protein 13 (ATG13), and ATG101 (Menzies et al. 2017). Specifically, the energy status of the cell, as indicated by the ratio of adenosine monophosphate (AMP) over adenosine triphosphate (ATP), is sensed by the AMP-dependent protein kinase (AMPK), while the mTOR complex 1 (mTORC1) detects the availability of nutrients and growth factors. AMPK and mTORC1 additionally integrate input from a plethora of other cellular pathways, for instance the PI3K-AKT pathway (for details, see 1.2.3). Both kinases regulate the ULK1 complex via phosphorylation in an opposing manner (Alers et al. 2012a; Egan D et al. 2011). Low cellular energy levels activate AMPK through phosphorylation (Thr172) or allosteric binding of AMP, leading to the direct activation of ULK1 by phosphorylation at different sites (Ser317, Ser555, Ser777) (Egan D et al. 2011; Kim et al. 2011). In contrast, growth factor signaling and ample amino acid availability trigger mTORC1 activation, resulting in inhibition of ULK1 either by direct binding or ULK1 phosphorylation (Ser757), which prevents AMPK from activating ULK1 and additionally leads to the suppression of ULK1 kinase function 
(Ganley et al. 2009; Hosokawa et al. 2009; Jung et al. 2009; Kim et al. 2011; Laplante and Sabatini 2012; Menzies et al. 2017).

The active ULK1 complex then initiates the PI3K complex comprised of vacuolar protein sorting 34 (VPS34), VPS15, ATG14L, and Beclin 1 (Figure 3) (Menzies et al. 2015). The PI3K complex localizes to pre-autophagosomal initiation sites, the exact origin of which is still debated; the Golgi complex, mitochondria, the plasma membrane, and the endoplasmic reticulum (ER) are discussed as possible membrane sources amongst others (Menzies et al. 2015; Nikoletopoulou et al. 2015; Stavoe and Holzbaur 2019). The PI3K complex is responsible for the generation of phosphatidylinositol 3-phosphate (PI3P). The detailed function of PI3P remains to be elucidated; yet, it is deemed important for the recruitment of downstream autophagic proteins to the growing phagophore membrane (Menzies et al. 2017).

Hereafter, a series of steps is executed to mediate elongation of the phagophore and ultimately form a complete autophagosome by membrane closure (Figure 3). Two interconnected ubiquitin-like conjugating systems, the ATG12-ATG5-ATG16L1 system and the microtubule-associated protein 1 light chain 3 (LC3)/ATG8 system, play major roles in this process (Wang Y et al. 2018). ATG12 is firstly conjugated to ATG5 through enzymatic activity of ATG10 and ATG7 (Stavoe and Holzbaur 2019). The resulting ATG5-ATG12 complex binds to ATG16L1 to form the ATG12-ATG5-ATG16L1 complex, which then associates with pre-autophagosomal membranes and assists with their elongation through recruitment of LC3 (Menzies et al. 2017). LC3 is firstly C-terminally cleaved by ATG4B to form LC3-I. LC3-I is then conjugated to phosphatidylethanolamine (PE) through a mechanism involving ATG7, ATG3, and ATG12-ATG5-ATG16L1, leading to the generation of LC3-II. LC3-II is strongly associated with autophagosomal membranes and mediates membrane elongation (Menzies et al. 2017; Wang Y et al. 2018). Additionally, receptors that label cargo, such as p62/ sequestosome 1 (SQSTM1), contain LC3-interacting (LIR) motifs and ubiquitin-associated domains (UBA), functioning as a bridge to enhance the incorporation of cargo into the growing autophagosome (Bjorkoy et al. 2005; Menzies et al. 2017). This cascade thus concomitantly sustains expansion of the phagophore membranes and engulfment of cargo, finally resulting in the closure of mature autophagosomes. It is worthy to note that the ATG12-ATG5-ATG16L1 complex dissociates after autophagosome completion, while LC3-II remains on the membrane of mature autophagosomes (Nikoletopoulou et al. 2015; Wang Y et al. 2018). LC3-II is thus used as a direct marker for autophagosome biogenesis and autophagic activity (Klionsky et al. 2016). Similarly, the 
incorporation and subsequent degradation of p62 via autophagy makes it possible to use p62 as an indirect marker of autophagy, as its levels inversely correlate with autophagy activation (Klionsky et al. 2016).

The complete autophagosome is then trafficked along microtubules to the perinuclear region in order to enable fusion with lysosomes and/or endosomes, resulting in the formation of autolysosomes or amphisomes, respectively (Figure 3) (Menzies et al. 2015; Nikoletopoulou et al. 2015). Mechanistically, the fusion is mediated via a myriad of non-ATG proteins, including ATPases, Rab proteins, members of the soluble N-ethylmaleimide-sensitive factor attachment protein receptor family (SNAREs), and the endosomal sorting complex (Nikoletopoulou et al. 2015). Finally, the acidic environment after fusion makes it possible for lysosomal enzymes such as hydrolases and cathepsins to degrade and recycle the engulfed cargo (Bar-Yosef et al. 2019).

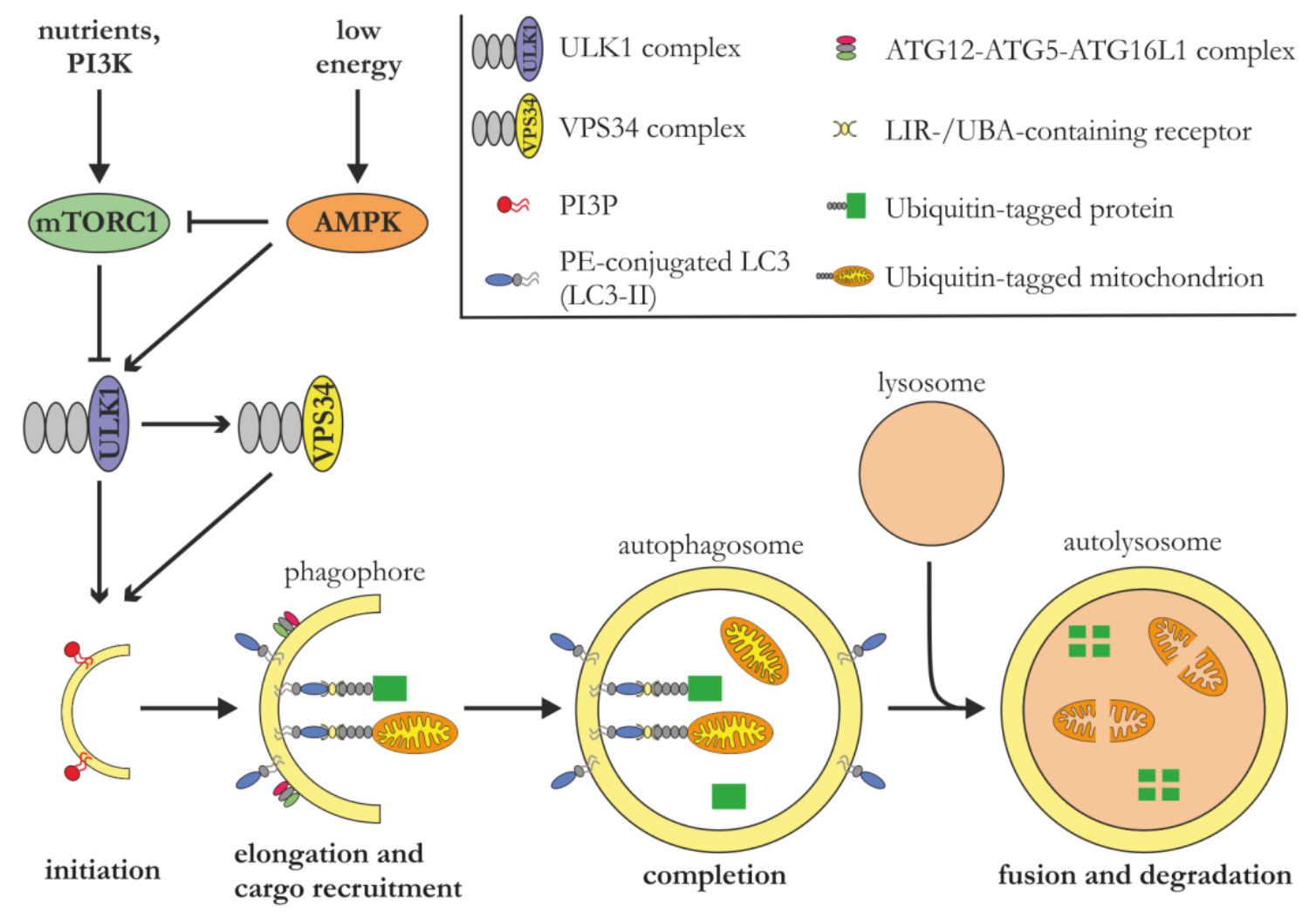

Figure 3: Schematic overview of mammalian ULK1-dependent autophagy. Activation of AMPK or inhibition of mTORC1 via integration of various signals that indicate metabolic status lead to autophagy initiation by formation of the ULK1 complex. The ULK1 complex then triggers the activation of the VPS34 complex. Both complexes together initiate the formation of pre-autophagosomal structures originating from different membrane sources, including the recruitment of PI3P. In a cascade of downstream events, PI3P assists in the recruitment of two different ubiquitin-like conjugating systems, the ATG12-ATG5-ATG16L1 system and the microtubule-associated protein 1 light chain 3 (LC3)/ATG8 system. Both systems mediate progredient elongation of the phagophore membrane and cargo recruitment, for which LIR/UBA-containing receptors act as a molecular bridge to recruit ubiquitin-tagged cargo such as mitochondria. After completion, the 
autophagosome fuses with lysosomes to finally degrade the engulfed cargo. Figure adapted from Rubinsztein et al. (2015), licensed under a CC BY-NC-SA 3.0 license, http://creativecommons.org/licenses/by-nc-sa/3.0/.

\subsection{Uncoordinated 51-like kinase 1 (ULK1)}

ULK1 is a serine/threonine protein kinase with four homologs, namely ULK2, ULK3, ULK4, and serine/threonine kinase 36, of which only ULK1 and ULK2 are thought to be implicated in conventional autophagy (Zachari and Ganley 2017). While there is some functional redundancy, ULK1 is considered the predominant isoform involved in autophagy regulation (Alers et al. 2012b; Chan et al. 2007).

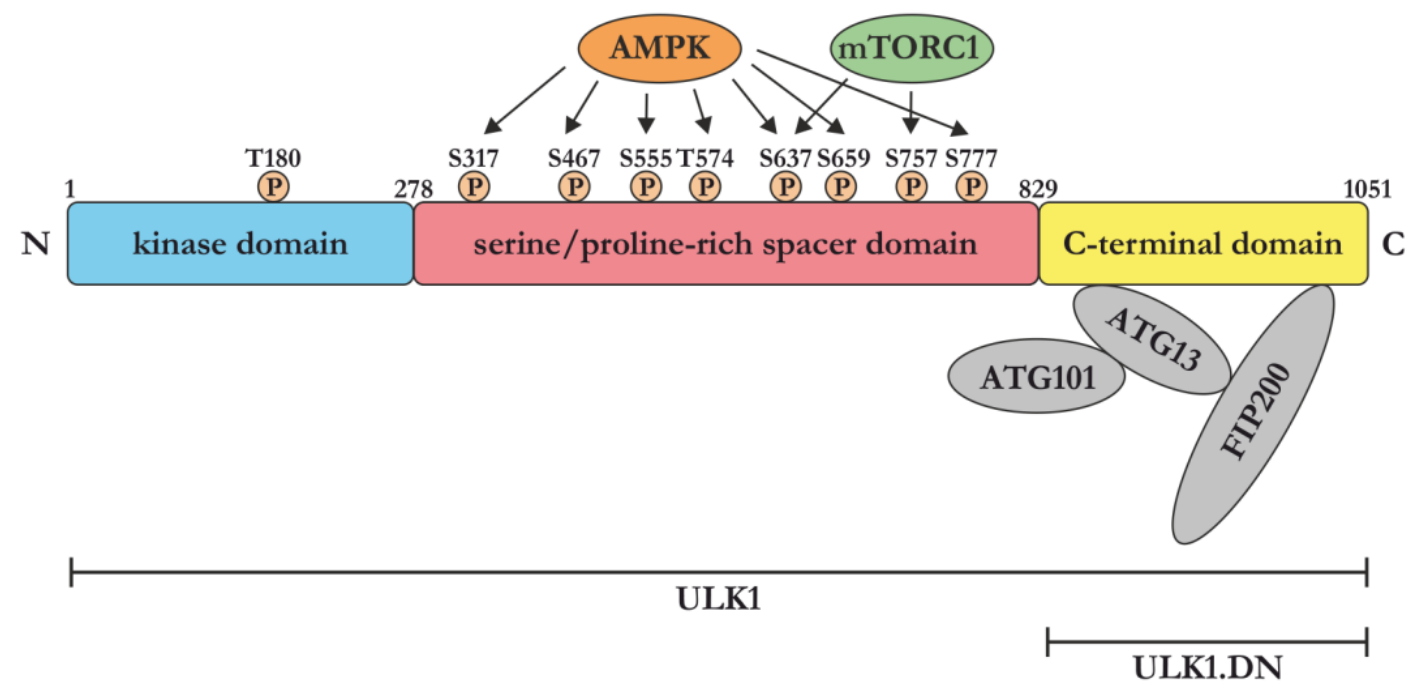

Figure 4: Detailed structure and phosphorylation sites of ULK1. Full-length ULK1 is comprised of an Nterminal kinase domain (amino acids 1 - 278), a serine/proline-rich domain (amino acids 279 - 828), and a Cterminal domain (amino acids 829 - 1051). ULK1 is regulated via autoregulation at its kinase domain, and by AMPK and mTORC1, which regulate multiple phosphorylation sites of the serine/proline-rich spacer domain. The C-terminal domain forms the ULK1 complex together with ATG13, FIP200, and ATG101, which is crucial for autophagy initiation. Overexpression of the C-terminal domain of ULK1 exerts dominant-negative properties (ULK1.DN) on autophagy. Figure adapted from Zhang et al. (2018), with kind permission from American Chemical Society.

In detail, ULK1 is comprised of an N-terminal kinase domain (amino acids 1 -278), an intermediate serine- and proline-rich (S/P) spacer domain (amino acids 279 - 828), and a Cterminal domain (CTD, amino acids 829 - 1051) (Figure 4) (Chan et al. 2007; Chan et al. 2009). The kinase domain is essential for ULK1-dependent autophagy induction, as kinasedead mutants as well as chemical inhibition of ULK1 enzymatic activity lead to a blockage of starvation-induced autophagic flux (Chan et al. 2007; Chan et al. 2009; Zachari and Ganley 2017). A plethora of autophagy-related targets of ULK1 phosphorylation have been described thus far, including ULK1 (autophosphorylation, T180), the ULK1 complex members ATG13, FIP200, and ATG101, and the PI3K complex members Beclin 1, VPS34, 
and ATG14L (Egan et al. 2015; Hosokawa et al. 2009; Jung et al. 2009; Lazarus et al. 2015; Zachari and Ganley 2017). For the regulation of ULK1 function, the S/P spacer domain is of crucial importance, as it is the site of multiple phosphorylation events depending on AMPK and mTORC1 activity (Figure 4) (Alers et al. 2012a; Egan DF et al. 2011; Kim et al. 2011). The CTD, on the other hand, has been found critical for membrane binding and the interaction with other proteins of the ULK1 complex (Chan et al. 2007; Chan et al. 2009).

Interestingly, overexpression of the CTD of ULK1 alone led to an evident dominantnegative effect (ULK1.DN) on starvation-induced autophagy (Chan et al. 2009). This dominant-negative effect was not mediated through altered membrane binding and could not be explained by the lack of the kinase domain alone, as the removal of a 7-residue motif (IERRLSA) in the last 14 amino acids of the overexpressed CTD reversed its dominantnegative properties (Chan et al. 2009). Hence, it was proposed that the CTD exerts dominant-negative properties on autophagy via regulation of an unknown downstream factor (Chan et al. 2009). Overexpression of the CTD of ULK1 therefore represents a useful approach to inhibit ULK1-dependent autophagy without affecting its direct binding partners.

\subsection{Autophagy and ULK1 in axonal degeneration and regeneration}

Autophagy has been widely implicated and extensively studied in neurodegenerative diseases. This is particularly due to the fact that in many neurodegenerative disorders, for example, Alzheimer's disease or Parkinson's disease (PD), intraneuronal aggregates of misfolded proteins, such as tau and $\alpha$-synuclein, are observed, which are degraded by autophagy (Menzies et al. 2015; Menzies et al. 2017). Furthermore, several key autophagic proteins have been shown to be mutated in various neurodegenerative diseases, leading to impaired autophagosome formation, disruption of cargo recognition, disrupted lysosomal function, or inhibited autolysosomal formation (Menzies et al. 2017). For instance, disease-causing mutations in SQSTM1 encoding p62 have been described in ALS (Teyssou et al. 2013), and mutations in the autophagolysosome pathway-associated genes glucorebrosidase $(G B A)$ and leucine-rich repeat kinase 2 ( $L R R K 2$ ) are found in PD (Hou et al. 2020). As a consequence, many experimental paradigms have largely aimed to rescue impaired autophagic flux in degenerative disorders, with autophagy activation being a widely used experimental approach (Rubinsztein et al. 2012; Rubinsztein et al. 2015). Also in models of acute axonal injury, such as optic nerve axotomy and traumatic brain injury, cytoprotective roles of autophagy and autophagy-inducing drugs have been described (Erlich et al. 2007; Rodriguez-Muela et al. 2012). 
Yet, as mentioned above (see 1.2.1), there is also evidence from models of acute axonal lesion that autophagy inhibition protects from lesion-induced AAD (Knoferle et al. 2010). Correspondingly, other studies have reported beneficial effects of autophagy suppression in models of traumatic neurodegeneration; for instance, through $A \operatorname{tg} 7$ downregulation (Yang et al. 2013). While the study by Knoferle et al. (2010) suggested autophagy as an executive mechanism of axonal degeneration, it left the detailed involvement of autophagic proteins in axonal degeneration after lesion unclear. However, a recent study (Ribas et al. 2015) shed some light on their temporal and spatial distribution after SCI. Here, LC3-positive autophagosomes, ATG5, and ULK1 were all found to accumulate in axonal bulbs after lesion, whereas ATG7 expression increased throughout the damaged axon (Figure 5). ATG5, ATG7, and ULK1 all peaked as early as $24 \mathrm{~h}$ after injury, while the maximum of autophagosome accumulation was observed two weeks after lesion. Additionally, an early increase in the calpain-mediated cleavage of spectrin was seen, which was hypothesized to reflect axonal calcium influx. Interestingly, ULK1 expression was most pronounced in close vicinity $(400 \mu \mathrm{m})$ to the lesion site. This corresponds to the axonal area affected by AAD (for details, see 1.2.1), suggesting a crucial and early involvement of ULK1 in the process of axonal degeneration after axonal lesion, possibly in a calcium-dependent manner. Another study confirmed that autophagy initiation after SCI was mediated through an AMPK-ULK1dependent pathway and could additionally show that inhibition of autophagy by 3-MA or AMPK inhibition attenuated remote degeneration after spinal cord hemisection (Bisicchia et al. 2017).

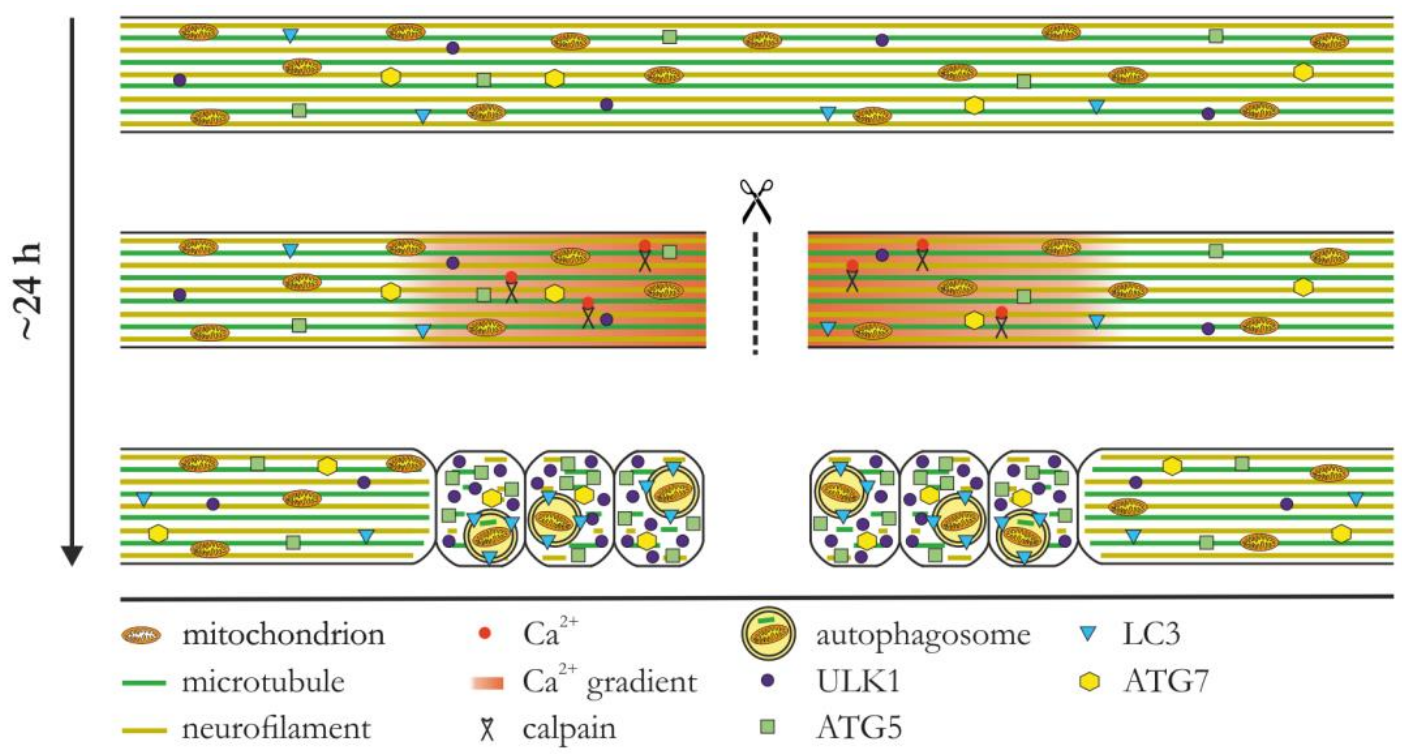

Figure 5: The involvement of autophagic proteins in axonal degeneration after axonal injury. A traumatic lesion to a CNS axon leads to rapidly increased intracellular calcium levels. Activation of calciumdependent enzymes such as calpain results in the disruption of microtubules and misalignment of 
neurofilaments. As a consequence of impaired axonal transport, local accumulation of organelles and subsequent formation of axonal bulbs becomes evident. While the distribution of autophagic proteins is fairly equal along the axon before axotomy, LC3-positive autophagosomes, ATG5, and particularly ULK1 accumulate in axonal bulbs within $24 \mathrm{~h}$ post-injury, while ATG7 shows a less localized intra-axonal increase. Figure adapted from Ribas et al. (2015), with kind permission from John Wiley and Sons.

These findings are of particular interest, as ULK1 was previously found implicated in the regulation of axon guidance in the developing mouse brain (Wang B et al. 2018). ULK1 also localized to growth cones and regulated neurite branching during sensory axon outgrowth (Zhou et al. 2007). In contrast, a high-throughput screen of kinases involved in neurite outgrowth using the human neuroblastoma line SH-SY5Y demonstrated that small interfering ribonucleic acid(siRNA)-mediated knock-down of ULK1 led to increased neurite outgrowth (Loh et al. 2008). Hence, the detailed role of ULK1 in axonal degeneration and regeneration, harmful or protective, is unclear and warrants further investigation. Specifically, the accumulation of ULK1 during axonal degeneration post-injury raises the question of whether ULK1-dependent autophagy is a crucial executor of axonal degeneration, the blockage of which could counteract axonal demise, or if it rather represents an endogenous axon-protective and possibly pro-regenerative mechanism that should be enhanced after axonal lesion.

\subsection{Methods to model axonal degeneration and regeneration after axonal injury in vitro}

Different models can be used to study axonal degeneration and regeneration after axonal injury in vitro. In principle, these models aim to induce axonal degeneration through a direct lesion to axons or by replicating the downstream signaling events known to occur in the axon post-injury. Experimental paradigms for both mechanical and pharmacological induction of axonal degeneration, namely calcium signaling and autophagy, will be briefly explained in the following, all of which are used in different experimental setups in this thesis.

\subsubsection{Mechanical induction of axonal degeneration via scratch lesion assays}

A fairly basic approach to model axonal degeneration in a dish is to perform a mechanical lesion to cultured neurons using a pipette tip (Roser et al. 2018; Tonges et al. 2011). It was previously demonstrated that key events of axonal degeneration observed in vivo, such as calpain-mediated cleavage of spectrin and CRMP2, could be replicated in vitro using this technique (Zhang et al. 2016). Even though the lesion affects both neurons and neurites, making it impossible to distinguish between effects on either cellular compartment, it provides a useful method to assess the gross changes of various proteins after lesion. 


\subsubsection{Modeling axonal degeneration through pharmacological modulation of calcium signaling and autophagy}

Another approach to model axonal degeneration in vitro is to apply drugs onto neurons, which induce pathways that were demonstrated to be upregulated in axonal degeneration after lesion in vivo. Mimicking the increased intra-axonal calcium influx seen post-lesion, the calcium ionophore A23187 can be added to neuronal cultures, which was shown to exacerbate axonal degeneration after rat ONC (Knoferle et al. 2010). In order to replicate the observed autophagy induction after axonal injury, autophagy can be activated in vitro through administration of the mTOR inhibitor rapamycin (Klionsky et al. 2016)

\subsubsection{Selective axonal lesions in microfluidic culture platforms}

The invention of microfluidic chambers (Park et al. 2006; Taylor et al. 2005) has revolutionized the studies on axonal degeneration in vitro. A microfluidic chamber is a culture platform consisting of four wells, two of which are linked by a main channel (Figure 6A). These two main channels are interconnected by small microgrooves $(7 \mu \mathrm{m}$ in width, $3 \mu \mathrm{m}$ in height, and $450 \mu \mathrm{m}$ in length). Neuronal cells are seeded into one main channel. Using a higher volume of medium on the cell side creates a continuous flow of medium from the cell side through the microgrooves to the other channel, allowing to direct axon growth and separate axons from dendrites. After a few days in culture, it is then possible to perform selective axonal lesions to induce and study axonal degeneration and regeneration post-injury in vitro (Figure 6B).

A

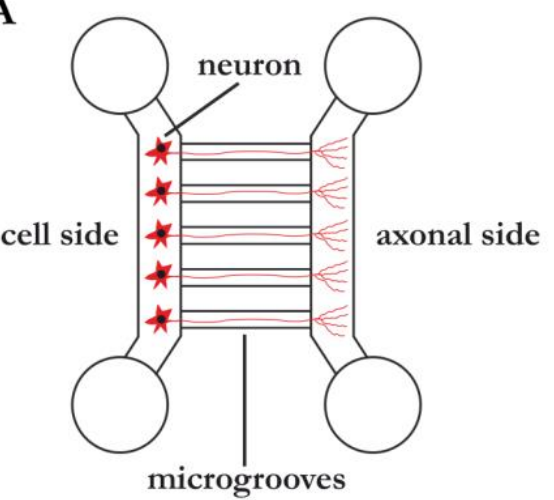

B
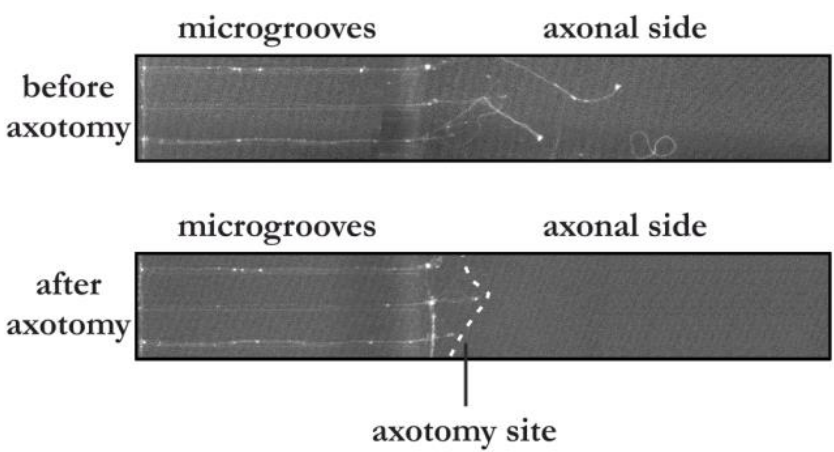

Figure 6: Overview of the microfluidic culture system. (A) Schematic overview of the microfluidic culture platform consisting of four wells, two of which are linked by a main channel. These two main channels are connected by small microgrooves. Neuronal cells are seeded into one main channel (cell side). After a few days in culture, axons grow through the microgrooves to the other main channel (axonal side). It is then possible to perform selective axonal lesions to induce and study lesion-induced axonal degeneration and regeneration in vitro. (B) Exemplary images showing axons of rat cortical neurons growing within the microgrooves and the axonal side before (top) and after axotomy (bottom). The axotomy site is indicated by a white dashed line. 


\subsubsection{Mimicking the inhibitory, anti-regenerative environment after lesion}

While neuronal monocultures provide the advantage of being able to study axonal degeneration and regeneration in detail, they do not allow to model the non-permissive environment produced by glial cells after axonal injury in vivo. To overcome this issue, a widely used approach is to culture neuronal cells on the growth-inhibitory substrate CSPG (Koch et al. 2014a; Koch et al. 2014b; Lingor et al. 2007; Niederost et al. 1999). This way, it is then possible to investigate pro-regenerative effects.

\subsection{Aims of this thesis}

Autophagy has been widely implicated in different models of axonal degeneration; however, its particular role, harmful or protective, seems to widely depend on the context. Intriguingly, the autophagy-initiating kinase ULK1 accumulated strongly and localized around the lesion site after SCI in previous experiments. This finding raised the question of whether ULK1dependent autophagy plays a beneficial or deleterious role after axonal injury. While it was already demonstrated that ULK1 is implicated in neurite outgrowth, the detailed role of ULK1 in axonal degeneration and regeneration has not been evaluated thus far. Earlier studies in the laboratory have demonstrated beneficial effects of unspecific autophagy inhibition on axonal degeneration. Therefore, this study aimed to investigate the effects of ULK1 inhibition on axonal degeneration and regeneration after axonal injury in vitro, hypothesizing that inhibition of ULK1-dependent autophagy could attenuate axonal degeneration and improve axonal regeneration (Figure 7).

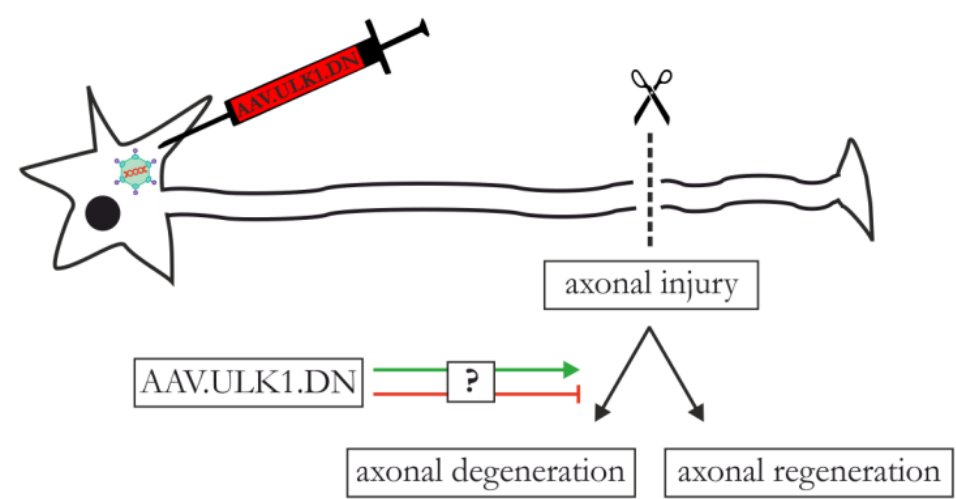

Figure 7: Schematic overview of the aims of this study. In this thesis, cortical neurons were transduced with an adeno-associated viral (AAV) vector expressing a dominant-negative form of the autophagy-initiating kinase ULK1 (ULK1.DN) in order to study the effect of ULK1 inhibition on axonal degeneration and regeneration after axonal injury in vitro.

As a prerequisite for all following experiments, the first aim of this thesis was to establish an in vitro method for ULK1 inhibition. For this, being considerate of the crucial role of ULK1- 
dependent autophagy for cell homeostasis, a moderate approach for the interference with ULK1 function was chosen rather than performing a complete knock-out or knock-down of ULK1. An adeno-associated viral (AAV) vector overexpressing the kinase-dead CTD of ULK1, which exerts dominant-negative properties (ULK1.DN) (Figure 4), was applied onto rat cortical neurons, and its transduction efficacy and toxicity were evaluated.

The second aim was then to establish an in vitro paradigm that mimics autophagy activation after axonal injury and, using this model, to evaluate the effect of ULK1.DN on autophagic proteins. It would be expected that successful treatment with ULK1.DN should attenuate ULK1-dependent autophagy.

Thirdly, if a significant modulation of autophagic proteins by ULK1.DN was observed, the effect of ULK1.DN on neurite outgrowth in both permissive and non-permissive environments, as well as its effect on AAD and axonal regeneration after selective axonal lesions in microfluidic chambers should be studied.

Finally, if significant effects on AAD, axonal regeneration, and/ or neurite outgrowth were found, the molecular mechanisms by which ULK1.DN regulates these processes should be investigated, including a detailed analysis of the several key proteins and pathways potentially involved. 


\section{Materials and Methods}

\subsection{Materials}

\subsubsection{Animals}

Primary cortical neuron cultures were prepared from embryonic day 18 (E18) Wistar rats obtained from the Central Animal Facility, University Medical Center Göttingen, Germany.

\subsubsection{Chemicals}

Table 1: Chemicals

\begin{tabular}{|l|l|}
\hline \multicolumn{1}{|c|}{ Chemicals } & \multicolumn{1}{c|}{ Company } \\
\hline 1-bromo-3-chlor-propane & $\begin{array}{l}\text { Sigma-Aldrich, St. Louis, MO, United } \\
\text { States }\end{array}$ \\
\hline $\begin{array}{l}\text { 4-(2-hydroxyethyl)-1- } \\
\text { piperazineethanesulfonic acid (HEPES) }\end{array}$ & AppliChem, Darmstadt, Germany \\
\hline Acetone & AppliChem, Darmstadt, Germany \\
\hline Acrylamide & AppliChem, Darmstadt, Germany \\
\hline Ammonium persulfate (APS) & $\begin{array}{l}\text { Sigma-Aldrich, St. Louis, MO, United } \\
\text { States }\end{array}$ \\
\hline B-27 supplement & $\begin{array}{l}\text { ThermoFisher Scientific, Waltham, MA, } \\
\text { United States }\end{array}$ \\
\hline Bafilomycin A1 & $\begin{array}{l}\text { Sigma-Aldrich, St. Louis, MO, United } \\
\text { States }\end{array}$ \\
\hline Bovine serum albumin (BSA) & AppliChem, Darmstadt, Germany \\
\hline Bromphenol blue & $\begin{array}{l}\text { Sigma-Aldrich, St. Louis, MO, United } \\
\text { States }\end{array}$ \\
\hline Calcium ionophore A23187 & $\begin{array}{l}\text { Sigma-Aldrich, St. Louis, MO, United } \\
\text { States }\end{array}$ \\
\hline Complete protease inhibitor & Roche, Basel, Switzerland \\
\hline CSPG & Merck, Darmstadt, Germany \\
\hline Deoxyribonuclease (DNase) I & $\begin{array}{l}\text { Sigma-Aldrich, St. Louis, MO, United } \\
\text { States }\end{array}$ \\
\hline Diethyl pyrocarbonate-treated water & $\begin{array}{l}\text { Sigma-Aldrich, St. Louis, MO, United } \\
\text { States }\end{array}$ \\
\hline Dithiothreitol (DTT) & $\begin{array}{l}\text { Sigma-Aldrich, St. Louis, MO, United } \\
\text { States }\end{array}$ \\
\hline Ethanol absolute (molecular biology grade) & AppliChem, Darmstadt, Germany \\
\hline
\end{tabular}




\begin{tabular}{|c|c|}
\hline Chemicals & Company \\
\hline Ethylenediaminetetraacetic acid (EDTA) & $\begin{array}{l}\text { Sigma-Aldrich, St. Louis, MO, United } \\
\text { States }\end{array}$ \\
\hline Fetal calf serum (FCS) & Merck, Darmstadt, Germany \\
\hline Glycerine & Carl Roth, Karlsruhe, Germany \\
\hline Glycine & AppliChem, Darmstadt, Germany \\
\hline Glycoblue $^{\mathrm{TM}}$ Coprecipitant & $\begin{array}{l}\text { ThermoFisher Scientific, Waltham, MA, } \\
\text { United States }\end{array}$ \\
\hline $\mathrm{H}_{2} \mathrm{O}_{2}$ & $\begin{array}{l}\text { Sigma-Aldrich, St. Louis, MO, United } \\
\text { States }\end{array}$ \\
\hline Hank's balanced salt solution (HBSS) & $\begin{array}{l}\text { ThermoFisher Scientific, Waltham, MA, } \\
\text { United States }\end{array}$ \\
\hline $\mathrm{HCl}$ & Carl Roth, Karlsruhe, Germany \\
\hline Isopropanol & AppliChem, Darmstadt, Germany \\
\hline L-glutamine & $\begin{array}{l}\text { ThermoFisher Scientific, Waltham, MA, } \\
\text { United States }\end{array}$ \\
\hline Laminin & $\begin{array}{l}\text { Sigma-Aldrich, St. Louis, MO, United } \\
\text { States }\end{array}$ \\
\hline Luminol & Merck, Darmstadt, Germany \\
\hline Methanol & AppliChem, Darmstadt, Germany \\
\hline $\mathrm{NaCl}$ & AppliChem, Darmstadt, Germany \\
\hline $\mathrm{NaHCO}_{3}$ & Merck, Darmstadt, Germany \\
\hline Neurobasal-A medium & $\begin{array}{l}\text { ThermoFisher Scientific, Waltham, MA, } \\
\text { United States }\end{array}$ \\
\hline Non-fat dried milk & AppliChem, Darmstadt, Germany \\
\hline Nonidet P-40 & AppliChem, Darmstadt, Germany \\
\hline P-coumaric acid & AppliChem, Darmstadt, Germany \\
\hline Penicillin/streptomycin/neomycin (PSN) & $\begin{array}{l}\text { ThermoFisher Scientific, Waltham, MA, } \\
\text { United States }\end{array}$ \\
\hline Phosphatase inhibitor & Roche, Basel, Switzerland \\
\hline Phosphate-buffered saline (PBS) & AppliChem, Darmstadt, Germany \\
\hline Poly-D-lysine (PDL) & $\begin{array}{l}\text { Sigma-Aldrich, St. Louis, MO, United } \\
\text { States }\end{array}$ \\
\hline Poly-L-ornithine (PLO) & $\begin{array}{l}\text { Sigma-Aldrich, St. Louis, MO, United } \\
\text { States }\end{array}$ \\
\hline $\begin{array}{l}\text { Precision Plus Protein Dual Color } \\
\text { Standards }\end{array}$ & Bio-Rad, Hercules, CA, United States \\
\hline Rapamycin & $\begin{array}{l}\text { Sigma-Aldrich, St. Louis, MO, United } \\
\text { States }\end{array}$ \\
\hline
\end{tabular}




\begin{tabular}{|l|l|}
\hline \multicolumn{1}{|c|}{ Chemicals } & \multicolumn{1}{c|}{ Company } \\
\hline Sodium dodecyl sulfate (SDS) & AppliChem, Darmstadt, Germany \\
\hline Staurosporine & $\begin{array}{l}\text { Sigma-Aldrich, St. Louis, MO, United } \\
\text { States }\end{array}$ \\
\hline Tetramethylethylenediamine (TEMED) & Carl Roth, Karlsruhe, Germany \\
\hline Transferrin & AppliChem, Darmstadt, Germany \\
\hline TRI-reagent & $\begin{array}{l}\text { Sigma-Aldrich, St. Louis, MO, United } \\
\text { States }\end{array}$ \\
\hline Tris base & AppliChem, Darmstadt, Germany \\
\hline Tris HCl & AppliChem, Darmstadt, Germany \\
\hline Trypsin & $\begin{array}{l}\text { Sigma-Aldrich, St. Louis, MO, United } \\
\text { States }\end{array}$ \\
\hline Tween-20 & AppliChem, Darmstadt, Germany \\
\hline
\end{tabular}

\subsubsection{Buffers, solutions, and cell culture medium}

Table 2: Buffers and solutions

\begin{tabular}{|c|c|}
\hline Buffer/solution/medium & Constituents \\
\hline Calcium magnesium-free (CMF) & $\begin{array}{l}450 \mathrm{~mL} \text { distilled } \mathrm{H}_{2} \mathrm{O}, 50 \mathrm{~mL} \text { 10x HBSS, } \\
700-800 \mu \mathrm{L} 7.5 \% \mathrm{NaHCO}_{3} \text { solution }\end{array}$ \\
\hline Cortex medium & $\begin{array}{l}\text { Neurobasal-A medium, } 0.5 \% 1 \mathrm{mg} / \mathrm{mL} \\
\text { transferrin, } 1 \% \text { PSN, } 0.25 \% \text { L-glutamine, } \\
2 \% \text { B-27 supplement }\end{array}$ \\
\hline $\begin{array}{l}\text { Enhanced chemiluminescence }(\mathrm{ECL}) \\
\text { reagent } 1\end{array}$ & $\begin{array}{l}10 \mu \mathrm{L} / \mathrm{mL} 250 \mathrm{mM} \text { luminol, } 4.4 \mu \mathrm{L} / \mathrm{mL} \\
90 \mathrm{mM} \text { p-coumaric acid, } 100 \mu \mathrm{L} / \mathrm{mL} 1 \mathrm{M} \\
\text { Tris } \mathrm{pH} 8.5 \text { in distilled } \mathrm{H}_{2} \mathrm{O}\end{array}$ \\
\hline ECL reagent 2 & $\begin{array}{l}0.6 \mu \mathrm{L} / \mathrm{mL} 30 \% \mathrm{H}_{2} \mathrm{O}_{2}, 100 \mu \mathrm{L} / \mathrm{mL} 1 \mathrm{M} \\
\text { Tris } \mathrm{pH} 8.5 \text { in distilled } \mathrm{H}_{2} \mathrm{O}\end{array}$ \\
\hline Gel electrophoresis buffer & $\begin{array}{l}192 \mathrm{mM} \text { glycine, } 0.1 \% \text { SDS, } 25 \mathrm{mM} \text { Tris- } \\
\mathrm{HCl}, \mathrm{pH} 8.3\end{array}$ \\
\hline Laemmli buffer & $\begin{array}{l}312.5 \mathrm{mM} \text { Tris } \mathrm{pH} 6.8,10 \% \text { SDS, } 50 \% \\
\text { glycerine, } 0.005 \% \text { bromphenol blue, } 100 \\
\text { mM DT'T }\end{array}$ \\
\hline Lysis buffer & $\begin{array}{l}0.5 \%(\mathrm{v} / \mathrm{v}) \text { Nonidet P-40, } 20 \mathrm{mM} \text { HEPES, } \\
300 \mathrm{mM} \mathrm{NaCl}, 5 \mathrm{mM} \text { EDTA, } 1 \mathrm{mM} \text { DTT, } \\
\text { including phosphatase inhibitor and } \\
\text { complete protease inhibitor }\end{array}$ \\
\hline PBS & $9.5 \mathrm{mg} / \mathrm{mL}$ PBS in distilled $\mathrm{H}_{2} \mathrm{O}$ \\
\hline Running phase gel $(10 \%)$ & $\begin{array}{l}2.83 \mathrm{~mL} 30 \% \text { acrylamide, } 2.125 \mathrm{~mL} 4 \mathrm{x} \text { Tris } \\
\text { pH 8.8, } 3.54 \mathrm{~mL} \text { distilled } \mathrm{H}_{2} \mathrm{O}, 4.25 \mu \mathrm{L} \\
\text { TEMED, } 42.5 \mu \mathrm{L} 10 \% \text { APS }\end{array}$ \\
\hline
\end{tabular}




\begin{tabular}{|l|l|}
\hline \multicolumn{1}{|c|}{ Buffer/solution/medium } & \multicolumn{1}{c|}{ Constituents } \\
\hline Stacking phase gel & $\begin{array}{l}0.65 \mathrm{~mL} 30 \% \text { acrylamide, } 1.25 \mathrm{~mL} 4 \mathrm{x} \text { Tris } \\
\text { pH } 6.8,3.05 \mathrm{~mL} \text { distilled } \mathrm{H}_{2} \mathrm{O}, 5 \mu \mathrm{L} \\
\text { TEMED, 25 } \mu \mathrm{L} \mathrm{10 \%} \mathrm{APS}\end{array}$ \\
\hline Tris-buffered saline (TBS) & $\begin{array}{l}10 \mathrm{mM} \text { Tris } \mathrm{HCl}, 150 \mathrm{mM} \mathrm{NaCl} \text { in distilled } \\
\mathrm{H}_{2} \mathrm{O}\end{array}$ \\
\hline TBS-Tween-20 (TBS-T) & $0.1 \%$ Tween-20 in TBS, pH 7.6 \\
\hline Transfer buffer & $\begin{array}{l}192 \mathrm{mM} \text { glycine, } 20 \% \text { methanol, } 25 \mathrm{mM} \\
\text { Tris HCl, pH 8.3 }\end{array}$ \\
\hline Tris & $10 \mathrm{mM}$ Tris-buffered saline pH 8.0 \\
\hline
\end{tabular}

\subsubsection{Pre-made kits}

Table 3: Kits

\begin{tabular}{|l|l|}
\hline \multicolumn{1}{|c|}{ Kit } & \multicolumn{1}{c|}{ Company } \\
\hline $\begin{array}{l}\text { ToxiLight } \\
\text { BioAss Noy Kit }\end{array}$ & Lon-Destructive Cytotoxicity, Basel, Switzerland \\
\hline $\begin{array}{l}\text { Pierce } \\
\text { PM } \text { bicinchoninic acid (BCA) }\end{array}$ & $\begin{array}{l}\text { ThermoFisher Scientific, Waltham, MA, Kit } \\
\text { United States }\end{array}$ \\
\hline Sylgard 184 Silicone Elastomer Kit & $\begin{array}{l}\text { DowDuPont, Wilmington, DE, United } \\
\text { States }\end{array}$ \\
\hline
\end{tabular}

\subsubsection{Plasmids}

Table 4: Plasmids

\begin{tabular}{|l|l|}
\hline \multicolumn{1}{|c|}{ Plasmid } & \multicolumn{1}{c|}{ Source } \\
\hline pAAV-hSyn-mCherry \\
[Genbank ID: KT345943] & $\begin{array}{l}\text { provided by Uwe Michel, Dept. of } \\
\text { Neurology, University Medical Center } \\
\text { Göttingen }\end{array}$ \\
\hline pcDNA3.1-ULK1.CTD & $\begin{array}{l}\text { kind gift from Sharon A. Tooze, Francis } \\
\text { Crick Institute, London, United Kingdom }\end{array}$ \\
\hline pAAV-hSyn-ULK1.DN & $\begin{array}{l}\text { provided by Uwe Michel, Dept. of } \\
\text { Neurology, University Medical Center } \\
\text { Göttingen }\end{array}$ \\
\hline
\end{tabular}

\subsubsection{Antibodies}

Table 5: Primary antibodies

\begin{tabular}{|l|l|l|l|}
\hline \multicolumn{1}{|c|}{ Antibody } & \multicolumn{1}{|c|}{ Species } & \multicolumn{1}{c|}{$\begin{array}{c}\text { Catalog } \\
\text { number }\end{array}$} & \multicolumn{1}{c|}{ Company } \\
\hline anti-AKT & rabbit & 9272 & $\begin{array}{l}\text { Cell Signaling Technology, } \\
\text { Cambridge, United Kingdom }\end{array}$ \\
\hline
\end{tabular}




\begin{tabular}{|c|c|c|c|}
\hline anti-AMPK $\alpha$ & rabbit & 2532 & $\begin{array}{l}\text { Cell Signaling Technology, } \\
\text { Cambridge, United Kingdom }\end{array}$ \\
\hline Antibody & Species & $\begin{array}{l}\text { Catalog } \\
\text { number }\end{array}$ & Company \\
\hline anti-ATG5 & rabbit & AP1812b & $\begin{array}{l}\text { Abgent, San Diego, CA, United } \\
\text { States }\end{array}$ \\
\hline anti-ATG7 & rabbit & $2631 S$ & $\begin{array}{l}\text { Cell Signaling Technology, } \\
\text { Cambridge, United Kingdom }\end{array}$ \\
\hline anti-cleaved caspase 3 & rabbit & 9661 & $\begin{array}{l}\text { Cell Signaling Technology, } \\
\text { Cambridge, United Kingdom }\end{array}$ \\
\hline anti-CRMP2 & rabbit & 9393 & $\begin{array}{l}\text { Cell Signaling Technology, } \\
\text { Cambridge, United Kingdom }\end{array}$ \\
\hline anti-GAPDH & mouse & $5 \mathrm{G} 4$ & Hytest Ltd., Turku, Finland \\
\hline anti-GSK3 $\beta$ & mouse & 610201 & $\begin{array}{l}\text { Becton Dickinson, Franklin } \\
\text { Lakes, NJ, United States }\end{array}$ \\
\hline anti-LC3 & mouse & $5 \mathrm{~F} 10$ & nanoTools, Teningen, Germany \\
\hline anti-ERK1/2 & rabbit & 9102 & $\begin{array}{l}\text { Cell Signaling Technology, } \\
\text { Cambridge, United Kingdom }\end{array}$ \\
\hline anti-mTOR & rabbit & 04-385 & Merck, Darmstadt, Germany \\
\hline anti-myc-tag & rabbit & 2272 & $\begin{array}{l}\text { Cell Signaling Technology, } \\
\text { Cambridge, United Kingdom }\end{array}$ \\
\hline anti-p62 & rabbit & P0067 & $\begin{array}{l}\text { Sigma-Aldrich, St. Louis, MO, } \\
\text { United States }\end{array}$ \\
\hline anti-PAK2 & rabbit & $2608 S$ & $\begin{array}{l}\text { Cell Signaling Technology, } \\
\text { Cambridge, United Kingdom }\end{array}$ \\
\hline anti-p-AKT & rabbit & 9271 & $\begin{array}{l}\text { Cell Signaling Technology, } \\
\text { Cambridge, United Kingdom }\end{array}$ \\
\hline anti-p-AMPK $\alpha$ & rabbit & 2535 & $\begin{array}{l}\text { Cell Signaling Technology, } \\
\text { Cambridge, United Kingdom }\end{array}$ \\
\hline anti-p-CRMP2 & rabbit & CP2251 & $\begin{array}{l}\text { ECM Bioscience, Versailles, } \\
\text { KY, United States }\end{array}$ \\
\hline anti-p-GSK3 $\beta$ & rabbit & 5558 & $\begin{array}{l}\text { Cell Signaling Technology, } \\
\text { Cambridge, United Kingdom }\end{array}$ \\
\hline anti-p-JNK & mouse & 9255 & $\begin{array}{l}\text { Cell Signaling Technology, } \\
\text { Cambridge, United Kingdom }\end{array}$ \\
\hline anti-p-ERK1/2 & mouse & 9106 & $\begin{array}{l}\text { Cell Signaling Technology, } \\
\text { Cambridge, United Kingdom }\end{array}$ \\
\hline anti-p-mTOR & rabbit & 5536 & $\begin{array}{l}\text { Cell Signaling Technology, } \\
\text { Cambridge, United Kingdom }\end{array}$ \\
\hline anti-p-PTEN & rabbit & 9554 & $\begin{array}{l}\text { Cell Signaling Technology, } \\
\text { Cambridge, United Kingdom }\end{array}$ \\
\hline
\end{tabular}




\begin{tabular}{|l|l|l|l|}
\hline anti-p-S6 & rabbit & 2211 & $\begin{array}{l}\text { Cell Signaling Technology, } \\
\text { Cambridge, United Kingdom }\end{array}$ \\
\hline \multicolumn{1}{|c|}{ Antibody } & \multicolumn{1}{|c|}{ Species } & $\begin{array}{c}\text { Catalog } \\
\text { number }\end{array}$ & \multicolumn{1}{c|}{ Company } \\
\hline anti-p-STAT3 & rabbit & 9145 & $\begin{array}{l}\text { Cell Signaling Technology, } \\
\text { Cambridge, United Kingdom }\end{array}$ \\
\hline anti-PTEN & rabbit & 9559 & $\begin{array}{l}\text { Cell Signaling Technology, } \\
\text { Cambridge, United Kingdom }\end{array}$ \\
\hline anti-ROCK2 & goat & sc-1851 & $\begin{array}{l}\text { Santa Cruz, Dallas, TX, United } \\
\text { States }\end{array}$ \\
\hline anti-STAT3 & rabbit & sc-482 & $\begin{array}{l}\text { Santa Cruz, Dallas, TX, United } \\
\text { States }\end{array}$ \\
\hline anti-TAOK1 & rabbit & ab52097 & $\begin{array}{l}\text { Abcam, Cambridge, United } \\
\text { Kingdom }\end{array}$ \\
\hline anti-ULK1 & rabbit & A7481 & $\begin{array}{l}\text { Sigma-Aldrich, St. Louis, MO, } \\
\text { United States }\end{array}$ \\
\hline anti- $\beta$-tubulin & mouse & T4026 & $\begin{array}{l}\text { Sigma-Aldrich, St. Louis, MO, } \\
\text { United States }\end{array}$ \\
\hline
\end{tabular}

Table 6: Secondary antibodies

\begin{tabular}{|l|l|l|l|}
\hline \multicolumn{1}{|c|}{ Antibody } & \multicolumn{1}{|c|}{ Species } & \multicolumn{1}{c|}{$\begin{array}{c}\text { Catalog } \\
\text { number }\end{array}$} & \multicolumn{1}{c|}{ Company } \\
\hline $\begin{array}{l}\text { anti-goat horseradish } \\
\text { peroxidase (HRP) }\end{array}$ & donkey & sc-2020 & $\begin{array}{l}\text { Santa Cruz, Dallas, TX, United } \\
\text { States }\end{array}$ \\
\hline anti-mouse HRP & horse & $7076 \mathrm{P} 2$ & $\begin{array}{l}\text { Cell Signaling Technology, } \\
\text { Cambridge, United Kingdom }\end{array}$ \\
\hline anti-rabbit HRP & goat & $7074 \mathrm{P} 2$ & $\begin{array}{l}\text { Cell Signaling Technology, } \\
\text { Cambridge, United Kingdom }\end{array}$ \\
\hline
\end{tabular}

\subsubsection{Equipment}

Table 7: Equipment

\begin{tabular}{|l|l|}
\hline \multicolumn{1}{|c|}{ Equipment } & \multicolumn{1}{c|}{ Company/manufacturer } \\
\hline 24-well culture plate & Sarstedt, Nümbrecht, Germany \\
\hline 3D gyratory rocker Stuart SSM3 & $\begin{array}{l}\text { Cole-Parmer, Vernon Hills, IL, United } \\
\text { States }\end{array}$ \\
\hline 48-well culture plate & $\begin{array}{l}\text { ThermoFisher Scientific, Waltham, MA, } \\
\text { United States }\end{array}$ \\
\hline 96-well microtest plate & Sarstedt, Nümbrecht, Germany \\
\hline Analytical balance BL210S & Sartorius, Göttingen, Germany \\
\hline Balance LE6202S & Sartorius, Göttingen, Germany \\
\hline
\end{tabular}




\begin{tabular}{|c|c|}
\hline Blotting paper Whatman & GE Healthcare, Chicago, IL, United States \\
\hline Centrifuge $5418 \mathrm{R}$ & Eppendorf, Hamburg, Germany \\
\hline Equipment & Company/manufacturer \\
\hline $\mathrm{CO}_{2}$ incubator HERAcell 150i & $\begin{array}{l}\text { ThermoFisher Scientific, Waltham, MA, } \\
\text { United States }\end{array}$ \\
\hline Developing machine Cawomat 2000 IR & $\begin{array}{l}\text { CAWO solutions, Schrobenhausen, } \\
\text { Germany }\end{array}$ \\
\hline Electrophoresis power supply & GE Healthcare, Chicago, IL, United States \\
\hline Film cassette & GE Healthcare, Chicago, IL, United States \\
\hline Film Hyperfilm ECL & GE Healthcare, Chicago, IL, United States \\
\hline Forceps & Fine Science Tools, Heidelberg, Germany \\
\hline Freezer $\left(-20^{\circ} \mathrm{C}\right)$ & Bosch, Stuttgart, Germany \\
\hline Freezer $\left(-80^{\circ} \mathrm{C}\right)$ & $\begin{array}{l}\text { ThermoFisher Scientific, Waltham, MA, } \\
\text { United States }\end{array}$ \\
\hline Fridge $\left(+4^{\circ} \mathrm{C}\right)$ & Liebherr, Bulle, Switzerland \\
\hline Glass coverslips & $\begin{array}{l}\text { ThermoFisher Scientific, Waltham, MA, } \\
\text { United States }\end{array}$ \\
\hline Ice machine AF100 & $\begin{array}{l}\text { Scotsman Ice Systems, Vernon Hills, IL, } \\
\text { United States }\end{array}$ \\
\hline Incubator $\left(37^{\circ} \mathrm{C}, 60^{\circ} \mathrm{C}\right)$ & Heraeus, Hanau, Germany \\
\hline $\begin{array}{l}\text { Inverted fluorescence microscope Axiovert } \\
200 \mathrm{M}\end{array}$ & Carl Zeiss Microscopy, Jena, Germany \\
\hline $\begin{array}{l}\text { Inverted fluorescence microscope } \\
\text { DMI6000 B }\end{array}$ & Leica Microsystems, Wetzlar, Germany \\
\hline Inverted light microscope Axiovert 40C & Carl Zeiss Microscopy, Jena, Germany \\
\hline Laminar flow hood LaminAir HB 2448 & Heraeus, Hanau, Germany \\
\hline Master mold for microfluidic chambers & $\begin{array}{l}\text { fabricated by Sarah Köster, Institute for X- } \\
\text { Ray Physics, University of Göttingen }\end{array}$ \\
\hline Mini-PROTEAN Spacer Plates & Bio-Rad, Hercules, CA, United States \\
\hline Mini-PROTEAN Tetra-Cell system & Bio-Rad, Hercules, CA, United States \\
\hline Multimode microplate reader Spark 10M & Tecan, Männedorf, Switzerland \\
\hline Nitrocellulose transfer membrane & AppliChem, Darmstadt, Germany \\
\hline Parafilm “M” PM996 & Bemis, Neenah, WI, United States \\
\hline Pasteur pipette & Sarstedt, Nümbrecht, Germany \\
\hline Petri dish & Sarstedt, Nümbrecht, Germany \\
\hline pH electrode Blueline 15 & Xylem Inc., Rye Brook, NY, United States \\
\hline $\mathrm{pH}$ meter Basic Meter PB-20 & Sartorius, Göttingen, Germany \\
\hline Pipette controller accu-jet pro & BRAND, Wertheim, Germany \\
\hline
\end{tabular}




\begin{tabular}{|l|l|}
\hline Pipette tips TipOne & STARLAB, Hamburg, Germany \\
\hline Pipettes & Gilson, Middleton, WI, United States \\
\hline $\begin{array}{l}\text { Polyvinylidene difluoride (PVDF) } \\
\text { transfer membrane }\end{array}$ & \multicolumn{1}{c|}{ Company/manufacturer } \\
\hline Razor blade & $\begin{array}{l}\text { Hugo Herkenrath Edelstahlschmiede, } \\
\text { Solingen, Germany }\end{array}$ \\
\hline Safe-lock tubes & Eppendorf, Hamburg, Germany \\
\hline Scalpel & $\begin{array}{l}\text { Aspen Surgical, Caledonia, MI, United } \\
\text { States }\end{array}$ \\
\hline Scanner CanoScan LidE 200 & Canon Solutions \\
\hline Screw cap tubes & Sarstedt, Nümbrecht, Germany \\
\hline Serological pipette & Sarstedt, Nümbrecht, Germany \\
\hline Shaker ST5 & CAT, Staufen-Etzenbach, Germany \\
\hline Thermomixer comfort 1.5 mL & Eppendorf, Hamburg, Germany \\
\hline Ultrasonic bath RK 100 H & BANDELIN electronic, Berlin, Germany \\
\hline Ultrasonic homogenizer HD 2070 & BANDELIN electronic, Berlin, Germany \\
\hline Vortex mixer 7-2020 & neoLAB, Heidelberg, Germany \\
\hline Water bath 1003 & $\begin{array}{l}\text { GFL Gesellschaft für Labortechnik, } \\
\text { Burgwedel, Germany }\end{array}$ \\
\hline Water purification system Arium pro & Sartorius, Göttingen, Germany \\
\hline
\end{tabular}

\subsubsection{Software}

Table 8: Software

\begin{tabular}{|l|l|}
\hline \multicolumn{1}{|c|}{ Software } & \multicolumn{1}{c|}{ Company } \\
\hline AxioVision 4.9 & Carl Zeiss Microscropy, Jena, Germany \\
\hline CorelDRAW 2017 & Corel Corporation, Ottawa, Canada \\
\hline Endnote X9 & $\begin{array}{l}\text { Clarivate Analytics, Philadelphia, PA, } \\
\text { United States }\end{array}$ \\
\hline Excel 2016 & Microsoft, Redmond, WA, United States \\
\hline GraphPad Prism 7 & GraphPad Software, La Jolla, CA, USA \\
\hline ImageJ 1.50i & $\begin{array}{l}\text { National Institutes of Health, Bethesda, } \\
\text { MD, United States }\end{array}$ \\
\hline $\begin{array}{l}\text { Leica Application Suite Advanced } \\
\text { Fluorescence 2.6.3 }\end{array}$ & Leica Microsystems, Wetzlar, Germany \\
\hline Windows 10 Paint & Microsoft, Redmond, WA, United States \\
\hline Word 365 & Microsoft, Redmond, WA, United States \\
\hline
\end{tabular}




\subsection{Methods}

\subsubsection{Cloning of plasmids and production of adeno-associated viral vectors}

Cloning of plasmids and production of AAV vectors was performed by Barbara Müller, Prof Dr Uwe Michel (both Dept. of Neurology, University Medical Center Göttingen), and Prof Dr Vinicius de Toledo Ribas (formerly Dept. of Neurology, University Medical Center Göttingen, now Institute of Biological Sciences, Federal University of Minas Gerais, Belo Horizonte, Brazil) according to a previously published protocol (Balke et al. 2020).

In brief, the previously described pcDNA3.1-ULK1.CTD (Chan et al. 2009) was used to clone ULK1.DN, which corresponds to the CTD of ULK1 (amino acids 829 to 1051) tagged with an N-terminal myc-tag, into the pAAV-hSyn-mCherry plasmid. Both the resulting plasmid from cloning pAAV-hSyn-ULK1.DN and the pAAV-hSyn-mCherry plasmid contain two human synapsin (hSyn) promoters. One hSyn promoter directs the expression of the reporter gene mCherry, while the second hSyn promoter leads to the expression of a non-coding transcript (Michel et al. 2002) in the control pAAV-hSyn-mCherry or of ULK1.DN in pAAV-hSyn-ULK1.DN, respectively. Both plasmids were then used to produce AAV vectors of the AAV pseudotype 1/2, which consists of an AAV2-derived genome packed into hybrid capsids of AAV1 and a mutated AAV2 capsid (Zhong et al. 2008), according to a previously published protocol (Zolotukhin et al. 1999). The resulting AAV vectors (Figure 8) are termed AAV.ULK1.DN and AAV.mCherry (control) in the following parts of this dissertation.

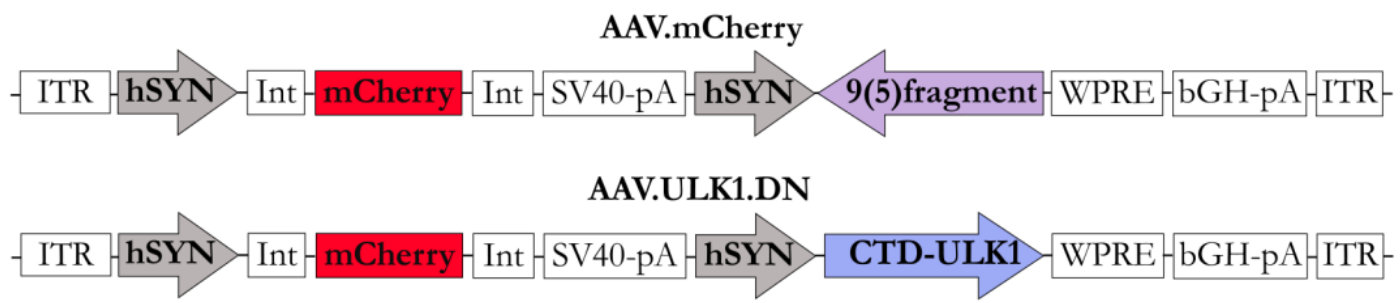

Figure 8: Adeno-associated viral vectors used in this thesis. Vector maps of the AAV vectors used to express the mCherry fluorophore and the CTD of ULK1, which has dominant-negative properties (ULK1.DN), or the non-translated 9(5)fragment (control), both of them expressed under the control of an hSyn promoter. ITR: AAV-2 inverted terminal repeat. Int: intron. SV40-pA: SV40-polyadenylation site. WPRE: woodchuck hepatitis virus posttranscriptional regulatory element. bGH-pA: bovine growth hormonepolyadenylation site. Figure adapted from Vahsen et al. (2020), licensed under a CC BY 4.0 license, https://creativecommons.org/licenses/by/4.0/. 


\subsubsection{Primary rat cortical neuron culture in culture plates}

Primary cortical neuron cultures were prepared with the approval of the governmental authorities and according to the legislation of the local animal research council of the State of Lower Saxony (Braunschweig), Germany.

\subsubsection{Coating with poly-L-ornithine and laminin}

For most experiments, cell culture plates were coated with PLO and laminin under sterile conditions in a laminar flow hood. PLO was diluted to a final concentration of $0.1 \mathrm{mg} / \mathrm{mL}$ in sterile double-distilled water $\left(\mathrm{H}_{2} \mathrm{O}\right) .500 \mu \mathrm{L}$ of this solution were added to each well on 24-well plates, while $300 \mu \mathrm{L}$ per well were used for 48 -well plates. After incubation at room temperature $(\mathrm{RT})$ overnight $(\mathrm{ON})$, the plates were washed two times with sterile doubledistilled $\mathrm{H}_{2} \mathrm{O}$. Afterwards, $500 \mu \mathrm{L}$ of laminin in sterile double-distilled $\mathrm{H}_{2} \mathrm{O}(1 \mu \mathrm{g} / \mathrm{mL})$ were added to each well on 24-well plates, while $300 \mu \mathrm{L}$ were used for 48 -well plates. After incubation at $37^{\circ} \mathrm{C}$ for a minimum of $2 \mathrm{~h}$, the laminin solution was aspirated, and the wells were washed two times with sterile double-distilled $\mathrm{H}_{2} \mathrm{O} .400 \mu \mathrm{L}$ of fresh cortex medium were added to each well and the plates were kept at $37^{\circ} \mathrm{C}$ until seeding of cells.

\subsubsection{Preparation of primary cortical neuron culture}

The preparation of embryos for cell culture was performed with the aid of Elisabeth Barski and Vivian Dambeck (both Dept. of Neurology, University Medical Center Göttingen) according to a previously described protocol (Zhang et al. 2016).

Briefly described, pregnant female E18 Wistar rats were sacrificed by $\mathrm{CO}_{2}$ intoxication and fixed on a preparation table. The front of the rat was disinfected with $70 \%$ ethanol. All embryos were extracted from the uterus and transferred to ice-cold CMF buffer in a laminar flow clean bench, so that all the following steps could be performed under aseptic conditions. After decapitation, the heads of the embryos were placed in a different dish filled with icecold CMF. Under a microscope, the brains were exposed and both hemispheres removed. After removal of the meninges, both cortices were separated from the other parts of the brain, dissected, and collected in a $10 \mathrm{~mL}$ tube with ice-cold CMF. The cortices of several embryos were pooled together and then centrifuged at $800 \mathrm{rpm}$ at RT for $2 \mathrm{~min}$. The supernatant was replaced with $1 \mathrm{~mL}$ trypsin $(25,000 \mathrm{U} / \mathrm{mL})$, and the tube was then incubated at $37^{\circ} \mathrm{C}$ in a water bath for $12 \mathrm{~min}$, after which $50 \mu \mathrm{L}$ of DNase $(5 \mathrm{mg} / \mathrm{mL})$ were added. After centrifugation at $800 \mathrm{rpm}$ and RT for $1 \mathrm{~min}$, the supernatant was immediately removed. The pellet was covered with $1 \mathrm{~mL}$ FCS to stop further trypsination. Using a $1000 \mu \mathrm{L}$ pipette and a handmade fire-polished Pasteur pipette, the pellet was gently triturated. The cell 
suspension was centrifuged at $800 \mathrm{rpm}$ for $4 \mathrm{~min}$. After removal of the supernatant, the resulting pellet was resuspended in $1 \mathrm{~mL}$ of warm culture medium. Neurons were counted using a Neubauer counting chamber. The appropriate number of cells as listed in Table 9 was diluted in $100 \mu \mathrm{L}$ of fresh, warm cortex medium and added to the culture plates, resulting in a final amount of $500 \mu \mathrm{L}$ medium per well. All plates were then kept at $37^{\circ} \mathrm{C}$ with $5 \% \mathrm{CO}_{2}$ and $95 \%$ humidity.

Table 9: Cell numbers in different cell culture experiments

\begin{tabular}{|l|c|c|c|}
\hline \multicolumn{1}{|c|}{ Purpose of experiment } & $\begin{array}{c}\text { Plate format } \\
{[\mathbf{w e l l ]}}\end{array}$ & $\begin{array}{c}\text { Cells per well } \\
{[\mathbf{n}]}\end{array}$ & $\begin{array}{c}\text { Volume of medium } \\
\text { per well }[\boldsymbol{\mu L} \text { ] }\end{array}$ \\
\hline Immunoblotting & 24 & 400,000 & 500 \\
\hline Proteomics/transcriptomics & 24 & 400,000 & 500 \\
\hline Neurite outgrowth & 24 & 20,000 & 500 \\
\hline Optimization of treatments & 48 & 200,000 & 500 \\
\hline
\end{tabular}

\subsubsection{Test of virus toxicity and transduction efficacy}

Both AAV.ULK1.DN and AAV.mCherry were tested on cortical neurons to evaluate virus toxicity and transduction efficacy. A minimum of $3 \mathrm{~h}$ after seeding of 400,000 cortical neurons in 24-well plates, both AAV vectors were diluted at increasing titers $\left(3 * 10^{6}\right.$ transducing units (TU), $1 * 10^{7} \mathrm{TU}, 3 * 10^{7} \mathrm{TU}, 1 * 10^{8} \mathrm{TU}$ ) in $100 \mu \mathrm{L}$ of fresh, pre-warmed cortex medium. For each well, the volume of medium was reduced to $200 \mu \mathrm{L}$, and cells were transduced by adding the viral vectors drop-wise to each well. On day in vitro (DIV) 1, each well was topped up with $200 \mu \mathrm{L}$ of cortex medium. On DIV 2, half of the total medium volume was removed and replaced with fresh cortex medium. Additional changes of half of the total medium volume were performed on DIV 4 and 6. On DIV 8, cells were imaged as described in 2.2.4.1 to quantify the transduction efficacy. Additionally, $200 \mu \mathrm{L}$ of medium from each well were stored at $-80^{\circ} \mathrm{C}$ until further utilization in a cell-toxicity assay as described in 2.2.5. Titers with high transduction efficacy but low toxicity were then chosen for all following experiments.

\subsubsection{Viral transduction and medium changes}

After the optimization of virus titers, in all following experiments, cortical neurons were transduced using the titers as listed in Table 10. Depending on the individual experimental design, viral vectors were added either on the day of preparation (DOP) or DIV 1. For each well, the volume of medium was reduced to $200 \mu \mathrm{L}$ prior to the addition of viral vectors. Neurons were transduced using $100 \mu \mathrm{L}$ of cortex medium containing the viral vectors at the 
indicated titers. On the next day after transduction, $200 \mu \mathrm{L}$ of cortex medium were added to each well. On the following day, half of the total medium volume was removed and replaced with fresh cortex medium. Cortical neurons were then cultured for a total duration of 4 DIV or $7-8$ DIV. For more mature cultures, additional medium changes of half of the total volume were then performed every other day.

Table 10: Virus titers employed in different experimental paradigms

\begin{tabular}{|l|c|c|}
\hline \multicolumn{1}{|c|}{ Experiment } & AAV.mCherry [TU] & AAV.ULK1.DN [TU] \\
\hline Immunoblotting & $5 * 10^{6}$ & $9 * 10^{6}$ \\
\hline Proteomics/transcriptomics & $5 * 10^{6}$ & $9 * 10^{6}$ \\
\hline Neurite outgrowth & $3 * 10^{6}$ & $3 * 10^{6}$ \\
\hline
\end{tabular}

\subsubsection{Scratch lesion model}

For some cultures, a scratch lesion (Zhang et al. 2016) was performed to mechanically induce axonal degeneration. 400,000 transduced cortical neurons per well in 24-well plates were scratched in three vertical and three horizontal lines using a $200 \mu \mathrm{L}$ pipette tip. This lesion paradigm was executed $24 \mathrm{~h}, 6 \mathrm{~h}$ or $3 \mathrm{~h}$ before cell lysis on DIV $3 / 4$ or DIV $7 / 8$ as described in 2.2.9. In selected conditions, $250 \mu \mathrm{L}$ of medium were removed directly before the scratch lesion and replaced by $250 \mu \mathrm{L}$ of fresh, pre-warmed cortex medium containing Bafilomycin A1 (working concentration: $10 \mathrm{nM}$ ). Bafilomycin A1 acts as an inhibitor of vacuolar type $\mathrm{H}^{+}-$ ATPase, blocking the fusion of autophagosomes and lysosomes. This inhibition of the degradation of autophagosomes makes it possible to investigate autophagic flux (Klionsky et al. 2016).

\subsubsection{Optimization of treatment with calcium ionophore}

For some cultures, the calcium ionophore A23187 was used to pharmacologically induce axonal degeneration (Knoferle et al. 2010). First, different concentrations of calcium ionophore were tested using 48-well plates and 200,000 non-transduced cortical neurons per well. Calcium ionophore was prepared at different concentrations $(0.05 \mu \mathrm{M}, 0.1 \mu \mathrm{M}, 0.5 \mu \mathrm{M}$, $1.0 \mu \mathrm{M}, 2.5 \mu \mathrm{M}, 5.0 \mu \mathrm{M})$ in $500 \mu \mathrm{L}$ of cortex medium for each well and applied on DIV 4 . Two different treatment paradigms were tested during optimization: In a first approach, all medium was removed $5.5 \mathrm{~h}$ before cell lysis and entirely replaced with new medium containing calcium ionophore. After $30 \mathrm{~min}$, all medium containing the calcium ionophore was aspirated and again replaced with fresh cortex medium. In a second approach, the medium containing calcium ionophore was added $5 \mathrm{~h}$ before cell lysis and incubated for the entire duration. 


\subsubsection{Treatment with calcium ionophore}

For experiments on cells transduced with viral vectors, two different paradigms were chosen after optimization. 400,000 cortical neurons per well were cultured in 24-well plates. First, on DIV 4, all medium was removed from the wells and $500 \mu \mathrm{L}$ of fresh, pre-warmed cortex medium containing calcium ionophore $(2.5 \mu \mathrm{M})$ were added to each well. After $30 \mathrm{~min}$, all medium was removed and replaced with fresh, pre-warmed cortex medium. $5.5 \mathrm{~h}$ later, cell lysis was performed. In a second approach, on DIV 7, all medium was removed from the wells and calcium ionophore (100 nM) diluted in $500 \mu \mathrm{L}$ of fresh, pre-warmed cortex medium was added to each well. In selected conditions, Bafilomycin A1 (10 nM) was additionally added to the medium at the same time to investigate autophagic flux. Cells were lysed $6 \mathrm{~h}$ later.

\subsubsection{Optimization of treatment with rapamycin}

For some cultures, a treatment with rapamycin was performed. Rapamycin is widely used to induce autophagy by inhibiting the mTOR kinase (Klionsky et al. 2016). First, different titers and incubation times were tested using 24-well plates and 400,000 non-transduced cortical neurons per well. On DIV 7/8, $250 \mu \mathrm{L}$ of medium were removed from each well and replaced by $250 \mu \mathrm{L}$ of fresh, pre-warmed cortex medium containing rapamycin (working concentrations: $100 \mathrm{nM}, 250 \mathrm{nM}, 750 \mathrm{nM}$ ). Cells were lysed after $3 \mathrm{~h}, 6 \mathrm{~h}$, and $24 \mathrm{~h}$.

\subsubsection{Treatment with rapamycin}

For experiments on cells transduced with viral vectors, two different paradigms were chosen after optimization. 400,000 cortical neurons per well were cultured in 24-well plates. In a first approach, on DIV 4, $250 \mu \mathrm{L}$ of medium were removed from each well and replaced by $250 \mu \mathrm{L}$ of fresh, pre-warmed cortex medium containing rapamycin (working concentration: $250 \mathrm{nM})$. Cells were lysed $6 \mathrm{~h}$ later. In a second approach, on DIV 7, $250 \mu \mathrm{L}$ of medium were removed from each well and replaced by $250 \mu \mathrm{L}$ of fresh, pre-warmed cortex medium containing rapamycin (working concentration: $750 \mathrm{nM}$ ). 24 h later, cell lysis was performed.

\subsubsection{Treatment with staurosporine}

To evaluate a possible protective effect against cell death, staurosporine was added to the culture medium in selected conditions. Staurosporine acts as a non-selective kinase inhibitor and has been widely used to induce apoptosis (Koh et al. 1995). On DIV 7, $250 \mu \mathrm{L}$ of medium were removed from each well and replaced by $250 \mu \mathrm{L}$ of fresh, pre-warmed cortex medium containing staurosporine (working concentrations: $30 \mathrm{nM}, 100 \mathrm{nM}, 300 \mathrm{nM}$ ). $24 \mathrm{~h}$ later, $100-200 \mu \mathrm{L}$ of the culture medium were removed. Technical replicates of the same 
conditions were pooled and stored at $-80^{\circ} \mathrm{C}$ until further utilization for a cell-toxicity assay as described in 2.2.5.

\subsubsection{Paradigm for the evaluation of neurite outgrowth}

For the evaluation of neurite outgrowth, cortical neurons were cultured on the permissive substrate laminin as well as the non-permissive substrate CSPG. 24-well plates were coated with $\mathrm{PLO}$ as described in 2.2.2.1. After two washing steps with sterile double-distilled $\mathrm{H}_{2} \mathrm{O}$, twelve wells were coated with $250 \mu \mathrm{L}$ of laminin solution as described above. $250 \mu \mathrm{L}$ of CSPG $(1.25 \mu \mathrm{g} / \mathrm{mL}$ diluted in laminin solution) were added to the remaining twelve wells. All wells were incubated at $37^{\circ} \mathrm{C}$ with $5 \% \mathrm{CO}_{2}$ and $95 \%$ humidity for $2 \mathrm{~h}$. Hereafter, all wells were washed twice with sterile double-distilled $\mathrm{H}_{2} \mathrm{O} .400 \mu \mathrm{L}$ of fresh cortex medium were added to each well and the plates were placed in an incubator until seeding of cells. Cortical neurons were prepared as described in 2.2.2.2 and 20,000 cells were seeded into each well. A minimum of $4 \mathrm{~h}$ later, viral transduction was performed using the titers listed in Table 9 and as described in 2.2.2.4. Changes of half of the total medium volume were performed every other day. To quantify neurite outgrowth, images of the cultured neurons were taken on DIV 7 as described in 2.2.4.4.

\subsubsection{Primary rat cortical neuron culture in microfluidic chambers}

\subsubsection{Preparation and coating of coverslips}

Coverslips were sonicated in double-distilled $\mathrm{H}_{2} \mathrm{O}$ at $60^{\circ} \mathrm{C}$ for $30 \mathrm{~min}$. After washing with double-distilled water, they were cleaned and degreased by manual shaking in acetone for 5 min and washed with 100\% molecular biology ethanol for 5 min. Hereafter, every single coverslip was rinsed ten times with double-distilled $\mathrm{H}_{2} \mathrm{O}$. After drying at $60^{\circ} \mathrm{C}$ for $1 \mathrm{~h}$, the coverslips were transferred to a laminar flow hood, where they were wet with $100 \%$ molecular biology ethanol, flame-sterilized, and left to dry for a minimum of $1 \mathrm{~h}$. Placed in new sterile Petri dishes, each coverslip was coated with PDL diluted in sterile, double-distilled $\mathrm{H}_{2} \mathrm{O}(0.1 \mathrm{mg} / \mathrm{mL})$ and incubated at $37^{\circ} \mathrm{C}$ with $5 \% \mathrm{CO}_{2}$ and $95 \%$ humidity for $30 \mathrm{~min}$. After removal of the PDL solution and two washing steps with sterile double-distilled $\mathrm{H}_{2} \mathrm{O}$, the coverslips were left to dry for $1 \mathrm{~h}$.

\subsubsection{Fabrication and preparation of microfluidic chambers}

Microfluidic chambers were prepared according to the original protocol (Park et al. 2006; Taylor et al. 2005). Using a silicone elastomer kit, poly(dimethylsiloxane) prepolymer and cross-linker were mixed 10:1 and poured onto a master mold. The mold was cured at $60^{\circ} \mathrm{C}$ 
for 90 min until the mixture solidified. The silicone piece was carefully removed and cut with a razor blade, with special care to not damage the imprinted side where microgrooves and main channels are located. The silicon was punctured manually to create wells. Using tape, the chambers were cleaned thoroughly to remove dust particles that would otherwise interfere with the correct assembly of coverslips and chambers. Hereafter, they were placed in Petri dishes and transferred to a laminar flow hood. To clean the chambers, $70 \%$ molecular biology ethanol was poured into the dishes. After 5 min of gentle manual shaking, the ethanol was aspirated, and the chambers were left to dry for a minimum of $3 \mathrm{~h}$.

\subsubsection{Assembling of coverslips and microfluidic chambers}

To construct the whole microfluidic culture system, a dry microfluidic chamber was placed onto a dried coverslip with the microgrooves and channels facing downwards. Using gentle force with a pair of flat tweezers, the chamber was mounted onto the coverslip. The assembled system was pre-warmed at $37^{\circ} \mathrm{C}$ for $5 \mathrm{~min} .200 \mu \mathrm{L}$ of fresh pre-warmed cortex medium were added to one well near the entrance of one main channel and the system was incubated at $37^{\circ} \mathrm{C}$ for $10 \mathrm{~min}$. Using a light microscope, the microgrooves were checked to ensure that they were completely filled with medium, and $150 \mu \mathrm{L}$ of fresh, pre-warmed cortex medium were added to the second main channel. The chambers were then kept at $37^{\circ} \mathrm{C}$ until seeding of cortical neurons.

\subsubsection{Cell seeding, viral transduction and culturing}

Prior to seeding, all medium was aspirated from one main channel of the microfluidic chamber. Immediately afterwards, 210,000 cortical neurons, prepared as described in 2.2.2.2 and diluted in $75 \mu \mathrm{L}$ of cortex medium, were added close to the aperture of the main channel. The chambers were then incubated at $37^{\circ} \mathrm{C}$ with $5 \% \mathrm{CO}_{2}$ and $95 \%$ humidity for a minimum of $3 \mathrm{~h}$. Viral vectors (AAV.mCherry: $1.5 * 10^{6} \mathrm{TU}$; AAV.ULK1.DN: $4 * 10^{6} \mathrm{TU}$ ) were diluted in $100 \mu \mathrm{L}$ of fresh, pre-warmed cortex medium and added drop-wise to the cell side of each microfluidic chamber. On DIV 1, $200 \mu \mathrm{L}$ of fresh, pre-warmed cortex medium were added to the channel containing the neuronal cells, while $180 \mu \mathrm{L}$ were added to the other channel. On DIV 2, 3 and 5, half of the total volume of medium was removed from both main channels and replaced with fresh, pre-warmed cortex medium. $20 \mu \mathrm{L}$ more were added to the channel containing the cortical neurons to maintain a continuous medium flow. For more mature microfluidic chamber cultures, additional changes of half of the total medium were performed on DIV 7, 9 and 11, always maintaining a $20 \mu \mathrm{l}$ volume difference between both main channels. 


\subsubsection{Axotomy}

On DIV 7, axons were long enough to reach the end of the microgrooves and grew in to the second main channel, allowing to perform selective axonal lesions. Using a vacuum pump, axons were axotomized by aspirating all medium from the main channel on the axonal side for $5 \mathrm{~s}$. Passing air bubbles led to the mechanical lesion of axons, resulting in the induction of axonal degeneration. Immediately afterwards, the main channel was refilled with $150 \mu \mathrm{L}$ of fresh, pre-warmed cortex medium. The chambers were then imaged in a microscope incubation system as described in 2.2.4.2 and 2.2.4.3.

\subsubsection{Recycling of microfluidic chambers}

After completion of live-cell imaging experiments, the microfluidic chambers were re-cycled to enable reuse in further experiments. All medium was removed from the chambers and SDS $0.5 \%$ was added to the Petri dishes for $5 \mathrm{~min}$ to decontaminate AAV vectors. The solution was aspirated, and the chambers were washed ten times with $\mathrm{H}_{2} \mathrm{O}$. After five additional washing steps with double-distilled $\mathrm{H}_{2} \mathrm{O}$, the chambers were transferred to new Petri dishes and incubated at $37^{\circ} \mathrm{C}$ in a water bath $\mathrm{ON}$. After drying at $60^{\circ} \mathrm{C}$ for $1 \mathrm{~h}$, the chambers were stored in Petri dishes sealed with Parafilm until further utilization.

\subsubsection{Live-cell microscopy and quantification}

\subsubsection{Imaging and quantification of transduction efficacy}

To evaluate the efficacy of transduction with AAV.mCherry and AAV.ULK1.DN, cortical neurons seeded in culture plates and transduced with either vector were imaged on a Zeiss inverse microscope equipped with an incubation system $\left(37^{\circ} \mathrm{C}, 5 \% \mathrm{CO}_{2}\right)$. Using AxioVision software, photomicrographs were taken at 40x magnification. For a minimum of two wells per AAV vector, two view fields $(351 \mu \mathrm{m}$ x $278.1 \mu \mathrm{m})$ were imaged (bright-field and mCherry fluorescence). Using ImageJ, the number of cells expressing the viral vectors as determined by mCherry fluorescence was manually counted for each view field and divided by the total number of cells to calculate the transduction rate.

\subsubsection{Live-imaging and quantification of axonal degeneration in microfluidic chambers in} vitro

Cortical neurons seeded in microfluidic chambers and transduced with viral vectors were imaged with a Leica fluorescence microscope equipped with an incubation system $\left(37^{\circ} \mathrm{C}, 5 \%\right.$ $\mathrm{CO}_{2}$ ). Using Leica Application Software, photomicrographs of mCherry-fluorescent axons were taken at 16x magnification directly before and 5 min to $6 \mathrm{~h}$ after axotomy. For all time 
points, the Leica Application Suite was programed to automatically image the entire microgroove area of the chambers using multi-panel image acquisition and the "auto-focus" function.

The number of axonal bulbs was quantified using Leica Application Suite for each time point. Axonal bulbs are hallmarks of axonal fragmentation and thus indicators of axonal degeneration (Coleman 2005; Knoferle et al. 2010; Zhang et al. 2016). For ten randomly chosen microgrooves per chamber with clearly axotomized axons, the number of axonal bulbs was manually counted in the axon area $400 \mu \mathrm{m}$ proximal to the lesion site (sub-divided into $0-100 \mu \mathrm{m}, 100-200 \mu \mathrm{m}, 200-300 \mu \mathrm{m}, 300-400 \mu \mathrm{m})$ in a blinded fashion. To determine the baseline of axonal bulbs before axotomy, the number of axonal bulbs was manually quantified within the same microgrooves (within $400 \mu \mathrm{m}$ proximal to the distal aperture) before axotomy. Only bulbs with a minimum diameter of $2 \mu \mathrm{m}$ were counted in order to not take rarely occurring, small mCherry aggregates into account for the evaluation. The number of bulbs before axotomy was subtracted from the number of bulbs for each time point to determine the number of newly formed bulbs after axonal lesion.

\subsubsection{Live-imaging and quantification of axonal regeneration in microfluidic chambers in} vitro

In additional cultures, cortical neurons seeded in microfluidic chambers and transduced with viral vectors were imaged at longer time points after axotomy to evaluate axonal regeneration. As described in 2.2.4.2, photomicrographs of mCherry-fluorescent axons were taken at 16x magnification directly before and $24 \mathrm{~h}$ to $96 \mathrm{~h}$ after axotomy.

Using Leica Application Software, ten adjacent microgrooves were chosen in the middle of each chamber, where axons were readily distinguishable and had clearly been axotomized. The number of axons growing out of these microgrooves before axotomy was counted manually. For the time points after axotomy, lines were manually drawn at different distances $(100 \mu \mathrm{m}, 200 \mu \mathrm{m}, 400 \mu \mathrm{m}, 600 \mu \mathrm{m}, 800 \mu \mathrm{m}, 1000 \mu \mathrm{m})$ from the microgroove aperture. The number of regenerating axons was determined by manually counting the number of axons crossing these lines for each distance and time point. To normalize for slightly varying axon numbers in each culture, a ratio between the number of regenerating axons after axotomy and the axon number before axotomy was calculated for each time point.

\subsubsection{Imaging and quantification of neurite outgrowth in vitro}

Cortical neurons seeded onto laminin- and CSPG-coated culture plates and transduced with viral vectors were imaged on a Leica fluorescence microscope equipped with an incubation 
system $\left(37^{\circ} \mathrm{C}, 5 \% \mathrm{CO}_{2}\right)$. Using Leica Application Software, 5 x 5 view fields $(800 \mu \mathrm{m} \times$ $600 \mu \mathrm{m})$ in the center of each well were imaged (bright-field and mCherry fluorescence) at 16x magnification with multi-panel image acquisition and the "auto-focus" function.

Using NeuronJ (Meijering et al. 2004), a neurite tracing plugin for ImageJ, the total neurite length was quantified manually on 2 view fields and then divided by the manually counted number of cells to determine the total neurite length per cell. Two to three technical replicates per condition were analyzed for each experiment. To account for variability in the absolute neurite length in each culture, the relative neurite length was determined by normalizing the absolute neurite length per cell in each condition to the absolute neurite length per cell of the laminin-coated control condition transduced with AAV.mCherry.

\subsubsection{Cell-toxicity assay}

To evaluate virus toxicity and to study cell survival, samples of medium were analyzed using a bioluminescence-based ToxiLight ${ }^{\mathrm{TM}}$ toxicity assay kit following the manufacturer's instructions. The cytotoxicity assay measures adenylate kinase (AK) activity in culture medium, which is release from damaged cells. AK converts adenosine diphosphate (ADP) to adenosine triphosphate (ATP). ATP-dependent light emission catalyzed by luciferase can then be measured using a luminometer. The level of light emission shows a linear correlation to the AK concentrations and thus the manifestation of cell death (Crouch et al. 1993).

Frozen medium samples were thawed to reach RT. $30 \mu \mathrm{L}$ of each sample were added in triplicates to the kit's 96-well test plate. The kit's reagents were thawed to reach RT and $30 \mu \mathrm{L}$ were added to each well to start the reaction. Exactly 5 min after addition of the reagent to the first well, light emission was measured using a microplate reader equipped with a luminometer. Blank-corrected values for each condition were then used for statistical analysis.

\subsubsection{RNA extraction}

Cortical neurons were transduced with AAV.ULK1.DN and AAV.mCherry and cultured for 8 DIV. RNA extraction for transcriptomic analysis was performed by Dr Lucas Caldi Gomes (formerly Dept. of Neurology, University Medical Center Göttingen, now Dept. of Neurology, Rechts der Isar Hospital of the Technical University Munich). Briefly described, cell lysis was performed using TRI-reagent. Hereafter, organic/aqueous phase-separation was achieved with 1-bromo-3-chlor-propane. After centrifugation at $4^{\circ} \mathrm{C}$ and $12,000 \mathrm{xg}$ for $15 \mathrm{~min}$, the aqueous phase was transferred to a new tube, and RNA precipitation was 
performed by addition of isopropanol and GlycoBlue ${ }^{\mathrm{TM}}$ Coprecipitant ON. After centrifugation at $4^{\circ} \mathrm{C}$ and $12,000 \mathrm{x}$ for $30 \mathrm{~min}$, the RNA pellet was washed twice with $75 \%$ ice-cold ethanol and reconstituted in diethyl pyrocarbonate-treated water.

\subsubsection{Differential gene expression and exon usage analyses}

Transcriptomic analyses allow for the large-scale experimental quantification of the transcriptome in an unbiased manner. In detail, differential gene expression analysis captures differences in the expression of entire genes, while differential exon usage analysis enables the assessment of individual exons and thus evaluation of splicing. Both analyses were performed in this thesis to determine the relative changes in gene expression and differential splicing between AAV.ULK1.DN and AAV.mCherry. Cortical neurons were transduced with both AAV vectors and cultured for 8 DIV (for details, see 2.2.2). After extraction of RNA as described in 2.2.6, the further experimental steps were performed by Dr Gabriela Salinas and Dr Orr Shomroni (both Next Generation Sequencing - Integrative Genomics Core Unit, Institute of Human Genetics, University Medical Center Göttingen).

In brief, the extracted RNA was first controlled for quality and integrity, and then fragmented, reverse-transcribed into complementary deoxyribonucleic acid (cDNA), and ligated to adapters for sequencing. The resulting libraries were amplified, quantified, pooled, and finally sequenced. Samples underwent quality control and were aligned to the reference genome Rattus Norvegicus (rn6 version 93) for mapping and normalization purposes. For differential exon usage analysis, reads were counted for individual exons and the exon usage was calculated as the expression of each exon with respect to the expression of other exons in the same gene. Candidate genes were filtered using a false discovery rate(FDR)-corrected P-value $<0.05$. To assess the differential expression of entire genes, the number of fragment counts were analyzed, and genes were filtered using an absolute $\log _{2}$ fold change (fc) $>1$ and FDR-corrected P-value $<0.05$.

\subsubsection{Bioinformatic analysis of transcriptomic data}

Further analysis of the transcriptomic data was performed with bioinformatical tools. Circos version 0.69 (Krzywinski et al. 2009) was used to visualize differentially expressed exons. To study the functions of genes with differential exon expression, the Database for Annotation, Visualization and Integrated Discovery (DAVID) version 6.8 (Huang da et al. 2009) was employed by performing functional annotation to gene ontology (GO) terms (Ashburner et al. 2000; Mi et al. 2017; The Gene Ontology Consortium 2017). Using an EASE score of 0.1 
and a minimum number of 2 counts, the gene enrichment and functional annotation module was applied for GO biological process, molecular function, and cellular component terms.

\subsubsection{Protein extraction}

Cortical neurons grown in culture plates were lysed to extract proteins for Western blot analysis and proteomics. During the lysis procedure, the culture plates were placed on ice to dampen metabolic processes in response to medium withdrawal. All medium was removed from the wells, and the cells were gently rinsed with $700 \mu \mathrm{L}$ of ice-cold PBS. $25-30 \mu \mathrm{L}$ of ice-cold lysis buffer were added to each well. Using a small cell scraper, the cells were detached from the plate and mixed with lysis buffer. The mixture was transferred to an Eppendorf cup and snap-frozen at $-80^{\circ} \mathrm{C}$. The cups were then slowly thawed on ice, vortexed thoroughly, and re-frozen at $-80^{\circ} \mathrm{C}$ in order to disrupt cells and release proteins through the formation of ice crystals. After an additional thaw-freeze-thaw cycle, the cups were sonicated at $4^{\circ} \mathrm{C}$ and 2 cycles $(40 \%)$ for $30 \mathrm{~s}$. The cups were then centrifuged at $4^{\circ} \mathrm{C}$ and $14.0 \mathrm{rpm}$ for $30 \mathrm{~min}$. Hereafter, the supernatants were transferred to new Eppendorf cups and stored at $-80^{\circ} \mathrm{C}$ until further utilization.

\subsubsection{Measurement of protein concentration}

For each sample, the protein concentration was determined to load specific and equal amounts of protein when performing Western blots. A BCA protein assay kit was used to analyze the protein concentrations. This method makes use of the fact that cuprous ions $\left(\mathrm{Cu}^{1+}\right)$ are produced in the reaction of proteins with alkaline $\mathrm{Cu}^{2+}$ (so-called biuret reaction). BCA is then capable of forming a complex with $\mathrm{Cu}^{1+}$ resulting in an intense purple color. This color is stable and increases proportionally over a broad working range of protein concentrations (Smith et al. 1985).

First, the BCA working reagents were diluted 1:50 and mixed well. BSA was diluted at standard protein concentrations $(0.15625-20 \mu \mathrm{g})$ in $200 \mu \mathrm{L}$ of working reagent and added to 96 -well microtest plates in duplicates. $1-2 \mu \mathrm{L}$ of protein samples were diluted in $200 \mu \mathrm{L}$ of working reagent and added to the plate in triplicates. The solutions were mixed manually in all wells, the plate was covered with acetate foil and incubated at $37^{\circ} \mathrm{C}$ for $30 \mathrm{~min}$. The absorbance at $562 \mathrm{~nm}$ was then measured for each sample using a microplate reader equipped with a photometer. The blank-corrected absorbance measured for the different BSA standard protein samples was averaged and plotted against their known concentrations using Excel in order to calculate a linear regression curve. Using the equation of this curve, the 
protein concentration of each sample was determined from their averaged blank-corrected absorbances.

\subsubsection{Quantitative proteomic profiling}

Quantitative proteomic profiling allows for the large-scale experimental identification and quantification of proteins in an unbiased manner. A proteomic analysis was therefore performed to determine the relative changes in the proteome between both AAV vectors used in this thesis. Cortical neurons were transduced with AAV.ULK1.DN and AAV.mCherry and cultured for 8 DIV (for details, see 2.2.2). Proteins were extracted from cell lysates, and the protein content was quantified as described in 2.2.9 and 2.2.10. In order to remove components of the lysis buffer that might interfere with protein identification, a precipitation with acetone was performed. For this, $100 \mu \mathrm{g}$ of protein lysates were mixed with pre-cooled acetone (v:v 1:3) and precipitated at $-20^{\circ} \mathrm{C} \mathrm{ON}$. After centrifugation at $4{ }^{\circ} \mathrm{C}$ and $16,000 \mathrm{x}$ g for $30 \mathrm{~min}$, the supernatants were discarded. The pellets were left to dry on ice and stored at $-20^{\circ} \mathrm{C}$ until further utilization.

The further experimental steps were performed by Prof Dr Henning Urlaub and Dr Christof Lenz (both Max Planck Institute for Biophysical Chemistry Göttingen and Dept. of Clinical Chemistry, University Medical Center Göttingen) using an established protocol (Atanassov and Urlaub 2013). First, $50 \mu \mathrm{g}$ of protein per sample were separated by gel electrophoresis. The protein areas were cut out, sliced, reduced, and alkylated. After ON digestion with trypsin, the resulting tryptic peptides were extracted from the gel, dried and kept at $-20^{\circ} \mathrm{C}$ until further analysis.

Protein digests were dissolved, separated by nanoscale liquid chromatography, and identified and quantified using nanoscale liquid chromatography coupled to tandem mass spectrometry (nanoLC-MS/MS). For protein identification, qualitative LC-MS/MS analysis was performed using a Top30 data-dependent acquisition method on two biological replicates per sample in order to construct a spectral library. These spectra were searched against the UniProtKB reference proteome for Rattus norvegicus (31,606 entries, revision 04-2016) augmented with a set of 51 known contaminants in the laboratory of Dr Christof Lenz and Prof Dr Henning Urlaub. Hereby, 2,440 proteins could be identified at an FDR of 1\%. To quantify protein expression, quantitative sequential window acquisition of all theoretical fragment-ion spectra (SWATH) analysis was performed with three technical replicates per biological replicate. 1988 proteins could be quantified at an FDR of 1\%, 122 of which were 
significantly regulated according to a two-sided t-test with permutation-based FDR assessment $(\mathrm{FDR}=0.1, \mathrm{~s} 0=0.05)$.

\subsubsection{Bioinformatical analysis of proteomic data}

Further data analysis of the proteomic data was performed using bioinformatical tools. Similar to the analysis of the transcriptomic data (see 2.2.8), DAVID version 6.8 was employed for the functional annotation of differentially regulated proteins to GO terms to study their functional relevance. Using an EASE score of 0.1 and a minimum number of 2 counts, the gene enrichment and functional annotation module was applied for GO biological process and cellular component terms. To analyze protein-protein interaction between the differentially regulated proteins, the Search Tool for the Retrieval of Interacting Genes/Proteins (STRING) version 10.5 (Szklarczyk et al. 2017) was employed with a minimum required interaction score of 0.4 .

\subsubsection{Sodium dodecyl sulfate-polyacrylamide gel electrophoresis}

Equal amounts of protein $(10-40 \mu \mathrm{g})$ from each sample were diluted in gel electrophoresis buffer and Laemmli buffer containing 10\% DTT to final volumes of $25 \mu \mathrm{L}$ per sample. The mixtures were denatured and reduced by 5 min incubation at $95^{\circ} \mathrm{C}$. Sodium dodecyl sulfatepolyacrylamide gel electrophoresis (SDS-PAGE) (Laemmli 1970) was then performed to separate proteins according to their molecular weight. 10-well and 15-well gels, consisting of a stacking gel to collect proteins of different sizes and a running gel to separate the proteins, were prepared manually. Depending on the weight of the proteins to be analyzed, different acrylamide concentrations were used for the running gel. For example, small proteins (e.g. $16 \mathrm{kDa}$ ) were separated using $14-15 \%$ acrylamide, while $12 \%$ gels were used for intermediate size proteins (e.g. $54 \mathrm{kDa}$ ) and 10\% for large proteins (e.g. $160 \mathrm{kDa}$ ). The gels were placed in an electrophoresis chamber filled with electrophoresis buffer, and the samples and protein ladders were loaded onto the different lanes of the gel. Electrophoresis was performed at $40 \mathrm{~V}$ for $30 \mathrm{~min}$, until the samples left the stacking gel. Hereafter, the voltage was increased to $100-130 \mathrm{~V}$. The electrophoresis was stopped, when the smallest band of the protein ladder reached the end of the gel.

\subsubsection{Western blot analysis and quantification}

After the separation of proteins by gel electrophoresis, Western blot analysis (Burnette 1981; Towbin et al. 1979) was performed to quantify the relative protein expression level. The gels were removed from the electrophoresis boxes, soaked with transfer buffer, and the stacking 
gels were detached. The gel was then carefully placed onto a transfer membrane. After covering with two sheets of filter paper and two sponge pads, the sandwich was inserted into a blotting cassette. PVDF or Nitrocellulose membranes were used for protein transfer following the manufacturer's instructions for each antibody. Prior to transfer, PVDF membranes were activated in 100\% methanol for 30 s. Nitrocellulose membranes were shortly rinsed with transfer buffer. The cassettes were inserted into a blotting chamber filled with ice-cold transfer buffer. The chamber was placed on ice and the transfer of proteins from the electrophoresis gel to the membrane was performed at $4^{\circ} \mathrm{C}$, either at $100 \mathrm{~V}$ for $90-120 \mathrm{~min}$ or at $20 \mathrm{~V} \mathrm{ON}$. Hereafter, the membranes were removed from the cassettes and cut according to the molecular weight of the protein to be analyzed. Following the manufacturer's instructions for each antibody, blocking was performed with 5\% non-fat dried milk in TBS-T or 5\% BSA in TBS-T for $1 \mathrm{~h}$ at RT. The blotting procedure was optimized for each antibody to reduce background signal. The membranes were incubated with primary antibodies at $4{ }^{\circ} \mathrm{C} \mathrm{ON}$ using the optimized concentrations as outlined in Table 11. After three 5 min-washing steps with TBS-T, the membranes were incubated with corresponding HRP-coupled secondary antibodies (anti-mouse, anti-rabbit, anti-goat; concentrations as outlined in Table 11, diluted in the same solution as their respective primary antibodies) at RT for $1 \mathrm{~h}$. After three 5 min-washing steps with TBS-T, ECL reagents were diluted 1:1, added to the membranes, and incubated for $1 \mathrm{~min}$. The membranes were positioned in an autoradiography cassette, transferred to a dark room, and exposed to X-ray films for different times until optimal visualization of protein bands was achieved. The films were manually developed using a developing machine and scanned using Paint. ImageJ software was used to quantify band intensities. Target protein band intensities were divided by the intensities of their respective loading control (glyceraldehyde 3-phosphate dehydrogenase (GAPDH) or $\beta$-tubulin). The intensities of all samples were then normalized to one respective control condition on the same blot.

Table 11: Antibodies and dilutions used for Western blotting

\begin{tabular}{|l|c|c|c|}
\hline Target protein & $\begin{array}{c}\text { Molecular } \\
\text { weight [kDa] }\end{array}$ & $\begin{array}{c}\text { Dilution primary } \\
\text { antibody }\end{array}$ & $\begin{array}{c}\text { Dilution secondary } \\
\text { antibody }\end{array}$ \\
\hline AKT & 60 & $1: 1,000$ & $1: 1,000$ \\
\hline AMPK $\alpha$ & 62 & $1: 1,000$ & $1: 1,000$ \\
\hline ATG5 & 30,56 & $1: 500$ & $1: 1,000$ \\
\hline ATG7 & 78 & $1: 400$ & $1: 1,000$ \\
\hline $\begin{array}{l}\text { cleaved } \\
\text { caspase 3 }\end{array}$ & 17 & $1: 500$ & $1: 1,000$ \\
\hline
\end{tabular}




\begin{tabular}{|c|c|c|c|}
\hline CRMP2 & $60-80$ & $1: 1,000$ & $1: 2,000$ \\
\hline ERK1/2 & 42,44 & $1: 2,000$ & $1: 1,000$ \\
\hline Target protein & $\begin{array}{c}\text { Molecular } \\
\text { weight [kDa] }\end{array}$ & $\begin{array}{c}\text { Dilution primary } \\
\text { antibody }\end{array}$ & $\begin{array}{c}\text { Dilution secondary } \\
\text { antibody }\end{array}$ \\
\hline GAPDH & 36 & $1: 50,000$ & $1: 4,000$ \\
\hline GSK3 $\beta$ & 46 & $1: 2,000$ & $1: 2,000$ \\
\hline LC3-I/II & 16,18 & $1: 1,000$ & $1: 1,000$ \\
\hline mTOR & 289 & $1: 1,000$ & $1: 1,000$ \\
\hline $\begin{array}{l}\text { myc-tag } \\
\text { (N-terminal) }\end{array}$ & 30 & $1: 1,000$ & $1: 1,000$ \\
\hline p62 & 62 & $1: 3,000$ & $1: 3,000$ \\
\hline PAK2 & 61 & $1: 1,000$ & $1: 1,000$ \\
\hline $\mathrm{p}-\mathrm{AKT}$ & 60 & $1: 1,000$ & $1: 1,000$ \\
\hline p-AMPK $\alpha$ & 62 & $1: 1,000$ & $1: 1,000$ \\
\hline p-CRMP2 & 70 & $1: 1,000$ & $1: 2,000$ \\
\hline p-ERK1/2 & 42,44 & $1: 2,000$ & $1: 1,000$ \\
\hline $\mathrm{p}-\mathrm{GSK} 3 \beta$ & 46 & $1: 1,000$ & $1: 2,000$ \\
\hline p-JNK & 46,54 & $1: 500$ & $1: 1,000$ \\
\hline p-mTOR & 289 & $1: 1,000$ & $1: 1,000$ \\
\hline p-PTEN & 54 & $1: 1,000$ & $1: 1,000$ \\
\hline p-S6 & 32 & $1: 1,000$ & $1: 1,000$ \\
\hline p-STAT3 & 86 & $1: 2,000$ & $1: 1,000$ \\
\hline PTEN & 54 & $1: 1,000$ & $1: 1,000$ \\
\hline ROCK2 & 160 & $1: 200$ & $1: 2,000$ \\
\hline STAT3 & 86,91 & $1: 2,000$ & $1: 1,000$ \\
\hline TAOK1 & 116 & $1: 1,000$ & $1: 1,000$ \\
\hline ULK1 & 150 & $1: 1,000$ & $1: 1,000$ \\
\hline$\beta$-tubulin & 55 & $1: 80,000$ & $1: 3,000$ \\
\hline
\end{tabular}

\subsubsection{Statistical analyses}

Statistical analyses were conducted using GraphPad Prism 7 software. To test single groups, one-sample t-tests were performed. Two groups were compared using two-tailed unpaired t-tests, and multiple group comparisons were done by one-way analysis of variance (ANOVA) with Dunnett's or Sidak's post-hoc test or two-way repeated measurement (RM) ANOVA with Sidak's post-hoc test. The statistical test and number of in vitro experiments used for each analysis are indicated in each figure legend. Data are presented as means \pm standard 
error of the mean (SEM). Differences were considered significant when $\mathrm{P}<0.05(* \mathrm{P}<0.05$; $* * \mathrm{P}<0.01 ; * * * \mathrm{P}<0.001 ;$ N.S.: not significant). 


\section{Results}

\subsection{Adeno-associated viral vector-mediated overexpression of a dominant-negative form of ULK1 in vitro}

In order to inhibit ULK1 activity, an AAV vector expressing a dominant-negative form of ULK1 and the fluorescent reporter mCherry (AAV.ULK1.DN) was used in this thesis (Figure 8). As a control, an AAV vector leading to the expression of a non-translated transcript (instead of ULK1.DN) and mCherry (AAV.mCherry) was employed (Figure 8). Both AAV vectors were used to transduce primary rat cortical neurons on DIV 1. Transduction with both vectors resulted in clearly detectable mCherry fluorescence on DIV 8 (Figure 9A). Quantification of the transduction rates (number of cells with mCherry fluorescence divided by the total cell number) showed similar values after transduction with both AAV.ULK1.DN $(78.0 \pm 6.4 \%)$ and AAV.mCherry $(77.7 \pm 5.6 \%)$ (Figure 9B). To validate the expression of ULK1.DN by AAV.ULK1.DN, Western blot analysis of its myctag, which is coupled to the N-terminus of ULK1.DN, was performed. Transduction with AAV.ULK1.DN resulted in clear myc-tag expression on DIV 8 (Figure 9C). These results therefore confirm the successful transduction with both AAV vectors in vitro.

A

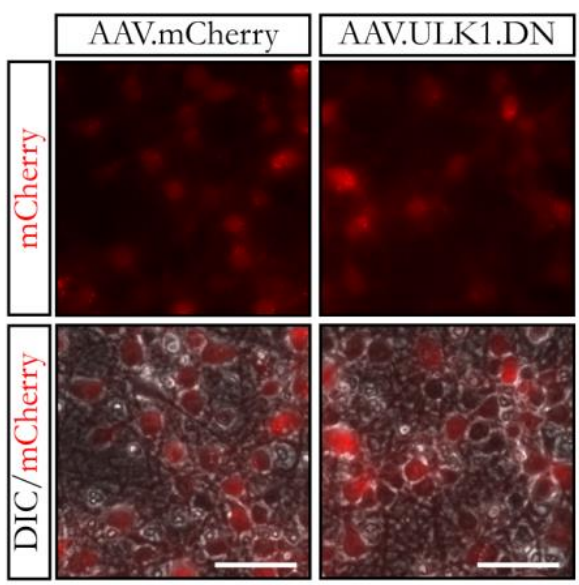

B

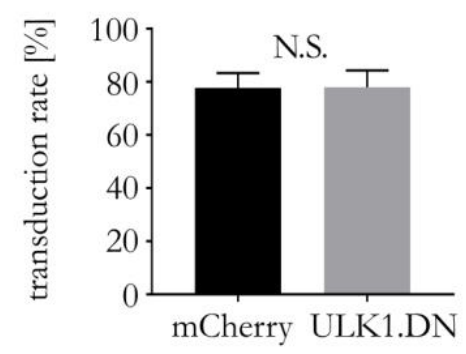

C

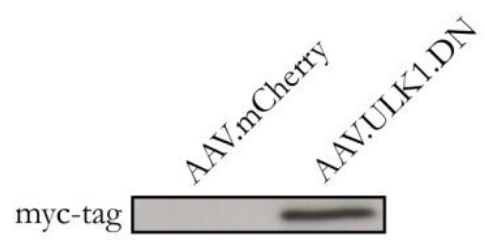

Figure 9: AAV.ULK1.DN-mediated overexpression of dominant-negative ULK1 in rat cortical neurons in vitro. Rat cortical neurons were transduced with AAV.mCherry and AAV.ULK1.DN on DIV 1, and imaged or lysed on DIV 8. (A) Exemplary images of rat cortical neurons transduced with given AAV vectors. Top: mCherry fluorescence, bottom: overlay of mCherry fluorescence with differential interference contrast (DIC) images. Scale bar: $50 \mu \mathrm{m}$. (B) Quantification of transduction rates (number of cells with mCherry fluorescence divided by total cell number) after transduction with given AAV vectors ( $\mathrm{n}=4$ independent cultures). Bars represent means \pm SEM. N.S.: No significant difference according to two-tailed unpaired t-test. (C) Representative Western blot after transduction with given AAV vectors showing myc-tag expression by AAV.ULK1.DN. Figure adapted from Vahsen et al. (2020), licensed under a CC BY 4.0 license, https://creativecommons.org/licenses/by/4.0/. 


\subsection{Evaluation of AAV vector toxicity}

To assess potential AAV vector toxicity in cell culture, cortical neurons were transduced with increasing titers of AAV.mCherry and AAV.ULK1.DN $\left(3 * 10^{6}\right.$ up to $1 * 10^{8}$ TU per well). On DIV 8, samples of the cell culture supernatant were used for a bioluminescence-based cytotoxicity assay for adenylate kinase (ToxiLight ${ }^{\mathrm{TM}}$ ). No significant differences were found between AAV.ULK1.DN and AAV.mCherry or by application of different titers (Figure 10), indicating equal cytotoxicity exerted by both AAV vectors.

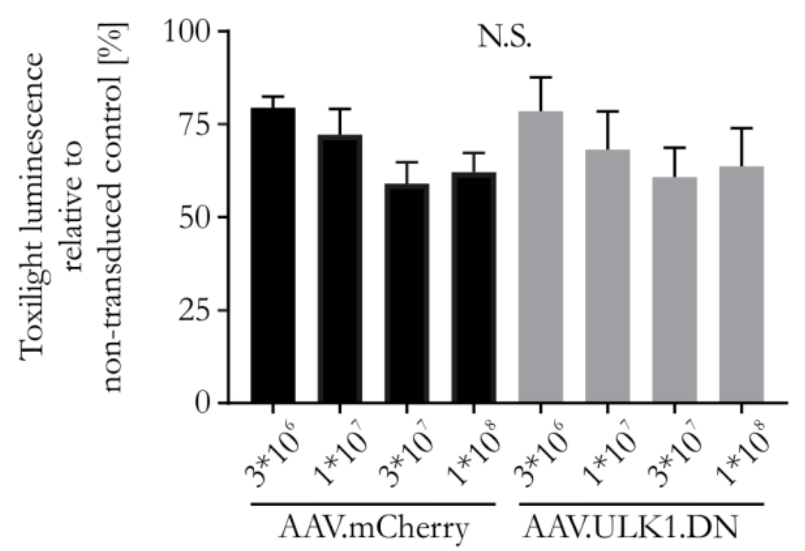

Figure 10: ToxiLight ${ }^{\mathrm{TM}}$ assay of cytotoxicity after transduction with different titers of AAV.mCherry and AAV.ULK1.DN. Rat cortical neurons were transduced with different titers of AAV vectors $\left(3 * 10^{6}\right.$ up to $1 * 10^{8} \mathrm{TU}$ per well). On DIV 8, the cell culture medium was used for a bioluminescence-based ToxiLight ${ }^{\mathrm{TM}}$ assay. The luminescence relative to non-transduced control shows no differences in cytotoxicity ( $\mathrm{n}=3$ independent cultures). Bars represent means \pm SEM. N.S.: No significant difference according to one-way ANOVA and Sidak's multiple comparisons test. Figure adapted from Vahsen et al. (2020), licensed under a CC BY 4.0 license, https://creativecommons.org/licenses/by/4.0/.

\subsection{Establishment of an in vitro model of autophagy induction and evaluation of the effect of AAV.ULK1.DN in this paradigm}

Having demonstrated the successful transduction with both AAV vectors in vitro, the next aim was to establish a reliable in vitro model of axonal degeneration mimicking the autophagy induction observed after SCI in a previous study in the laboratory (Ribas et al. 2015). In a second step, the effect of AAV.ULK1.DN on autophagic proteins should then be investigated using this model. For this, different methods and time points were evaluated: In a first approach, a mechanical scratch lesion was performed on cortical neurons to test lesioninduced autophagy induction. Secondly, cortical neurons were treated with the calcium ionophore A23187 to test calcium-induced autophagy activation. Lastly, a treatment with rapamycin was performed to pharmacologically induce autophagy. 


\subsubsection{Assessment of scratch lesion-mediated autophagy induction}

3.3.1.1 Analysis of autophagic markers after scratch lesion on DIV 3/4 and transduction with AAV.ULK1.DN

In a first approach, a scratch lesion was performed to induce autophagy through mechanical axonal lesion and study the effect of ULK1.DN on lesion-induced autophagy. Rat cortical neurons were transduced with AAV.ULK1.DN and AAV.mCherry, and scratch lesions were executed $24 \mathrm{~h}, 6 \mathrm{~h}$, and $3 \mathrm{~h}$ before lysis on DIV 4 (Figure 11A). Western blot analysis of LC3-II, used as a direct marker for autophagic activity, was performed to study the activation of autophagy after lesion (Figure 11B). No differences in LC3-II levels could be observed at any time point after scratch lesion in neurons transduced with AAV.mCherry (3 h: $0.84 \pm 0.08$ a.u.; 6 h: $0.92 \pm 0.09$ a.u.; 24 h: $0.95 \pm 0.19$ a.u.), indicating no effect of mechanical neuron transection on autophagy. Similarly, no difference could be detected after transduction with AAV.ULK1.DN (non-lesioned: $0.96 \pm 0.20$ a.u.; 3 h: $0.93 \pm 0.13$ a.u.; 6 h: $0.76 \pm 0.05$ a.u.; 24 h: $0.68 \pm 0.08$ a.u.).

A

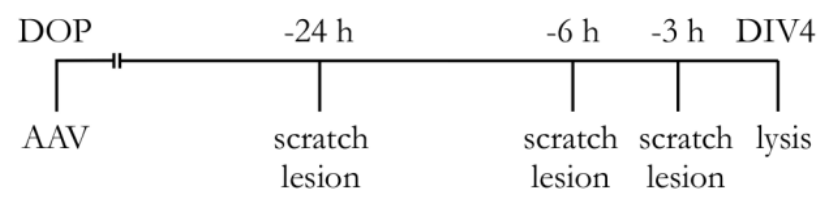

B $\frac{\text { AAV.mCherry }}{\text { CTR 3h 6h 24h }} \frac{\text { AAV.ULK1.DN }}{\text { CTR 3h 6h 24h }}$
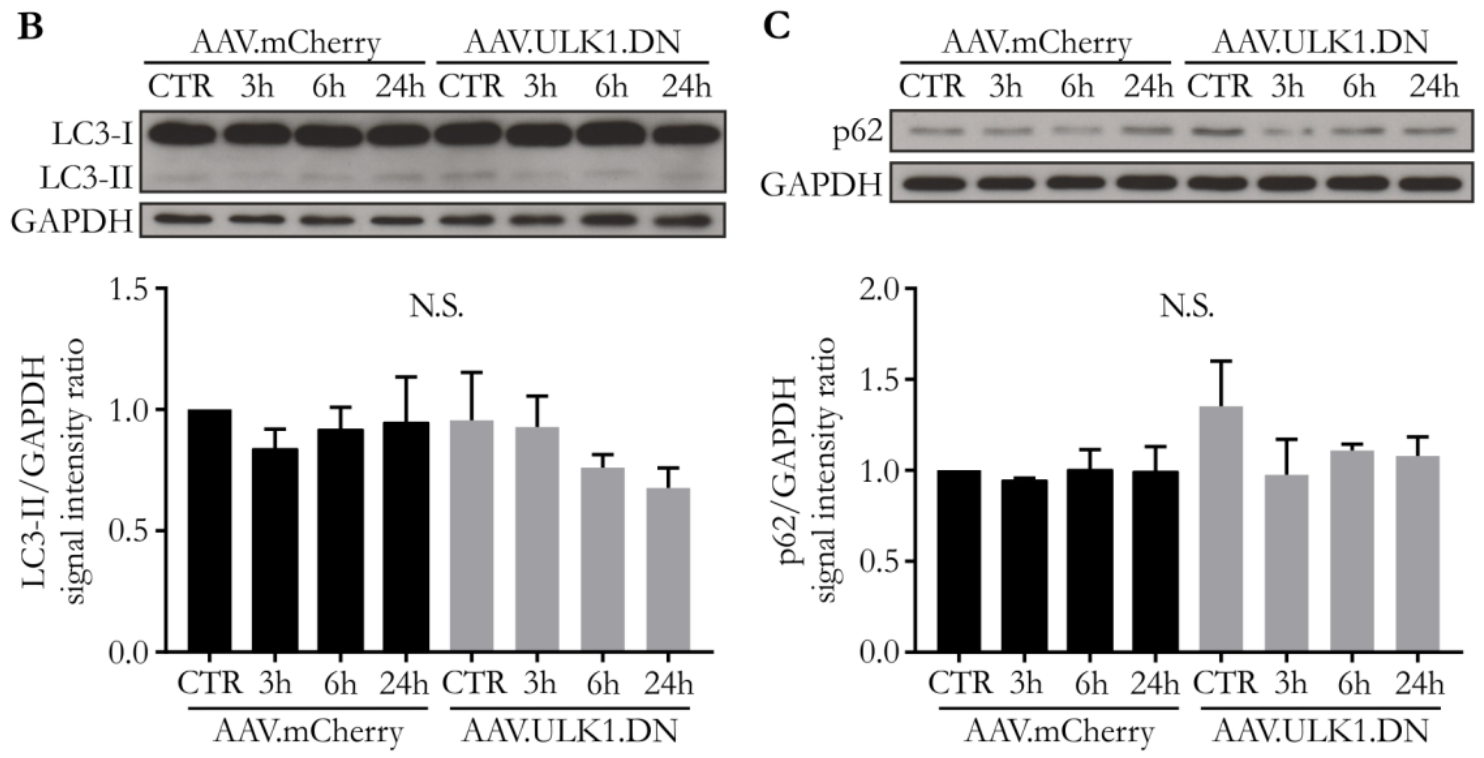

Figure 11: Autophagy markers after scratch lesion on DIV 3/4 and transduction with AAV.ULK1.DN. (A) Scheme of experimental setup. DOP: day of preparation of E18 rat cortical neurons. DIV: day in vitro. AAV: transduction with adeno-associated viral vectors. (B) Representative Western blots of LC3-I/II and GAPDH (as loading control) are shown at the top. CTR: non-lesioned control. Below, quantification of band intensities normalized to GAPDH ( $\mathrm{n}=5$ independent cultures). (C) Representative Western blots of p62 and GAPDH (as loading control) are shown at the top. Below, quantification of band intensities normalized to GAPDH ( $\mathrm{n}=3$ independent cultures). Bars represent means \pm SEM. N.S.: no significant difference, according to one-way ANOVA and Sidak's multiple comparisons test. 
Additionally, the expression of p62, which shows an inverse relation to autophagic activity, was assessed by Western blotting (Figure 11C). Cells transduced with AAV.mCherry showed no differences in p62 levels at any time point after scratch lesion ( 3 h: $0.94 \pm 0.02$ a.u.; 6 h: $1.00 \pm 0.12$ a.u.; 24 h: $0.99 \pm 0.14$ a.u.), corroborating the absence of any effect of mechanical neuron transection on autophagy. Similarly, no changes in p62 expression were detectable in neurons transduced with AAV.ULK1.DN (non-lesioned: $1.35 \pm 0.25$ a.u.; 3 h: $0.98 \pm 0.19$ a.u.; 6 h: $1.11 \pm 0.04$ a.u.; 24 h: $1.08 \pm 0.10$ a.u.).

Taken together, scratch lesions on DIV 3/4 did not result in a detectable induction of autophagy. Furthermore, no difference in the levels of autophagic proteins could be detected between cells transduced with AAV.mCherry and AAV.ULK1.DN.

3.3.1.2 Analysis of autophagic markers after scratch lesion on DIV 7 and transduction with AAV.ULK1.DN

As mechanical lesion at different time points on DIV $3 / 4$ did not lead to autophagy induction (see 3.3.1.1), it was tested next whether the execution of scratch lesions in more mature cultures (7 DIV) might result in autophagy activation. In this paradigm, cells were lysed only at $6 \mathrm{~h}$ after scratch lesion, as this time point showed evident upregulation of proteins involved in degeneration in previous experiments in the laboratory (Zhang et al. 2016). Additionally, cells were treated with bafilomycin, which blocks the fusion of autophagosomes and lysosomes, and thus allows for the more refined analysis of autophagic flux (Figure 12A).

Western blot analysis showed no effect of scratch lesions on LC3-II levels, neither in cells transduced with AAV.ULK1.DN nor AAV.mCherry (Figure 12B), indicating no effect of mechanical neuron transection on autophagy on DIV 7, either. The addition of bafilomycin resulted in a clear tendency to LC3-II accumulation in all conditions (AAV.mCherry: nonlesioned with bafilomycin: $5.27 \pm 1.53$ a.u.; lesioned with bafilomycin: $4.93 \pm 1.85$ a.u.; AAV.ULK1.DN: non-lesioned with bafilomycin: $6.28 \pm 2.71$ a.u.; lesioned with bafilomycin: $3.13 \pm 0.75$ a.u.), indicating the blockage of autophagic flux. However, all relevant comparisons were far from statistical significance and no difference was detectable between AAV.ULK1.DN and AAV.mCherry.

Similarly, the analysis of p62 levels showed no effect after scratch lesion in cells transduced with AAV.mCherry or AAV.ULK1.DN, neither in control conditions nor after bafilomycin treatment (Figure 12C), corroborating that mechanical neuron transection on DIV 7 had no detectable effect on autophagy. Transduction with AAV.ULK1.DN led to a tendency to increased p62 levels (non-lesioned: $1.41 \pm 0.38$ a.u.; non-lesioned with bafilomycin: 
$1.56 \pm 0.26$ a.u.; lesioned: $1.26 \pm 0.08$ a.u.; lesioned with bafilomycin: $1.15 \pm 0.12$ a.u.) compared to cells transduced with AAV.mCherry (non-lesioned with bafilomycin: $1.01 \pm 0.10$ a.u.; lesioned: $0.77 \pm 0.15$ a.u.; lesioned with bafilomycin: $0.80 \pm 0.05$ a.u.), but all comparisons were far from statistical significance.

Taken together and corresponding to the results on DIV 4, scratch lesions on DIV 7 did not lead to a reproducible induction of autophagy. Furthermore, no difference in the expression of autophagic markers could be detected between AAV.mCherry and AAV.ULK1.DN.

A

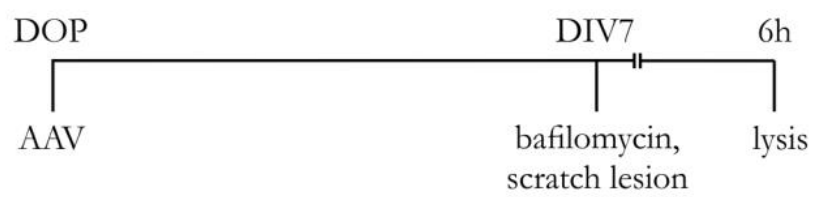

B
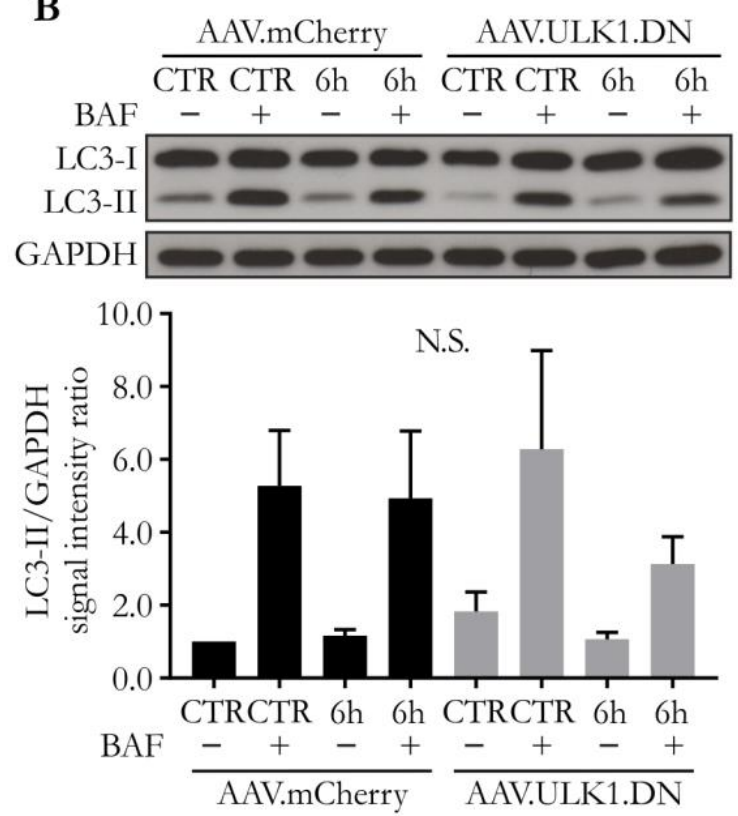

C
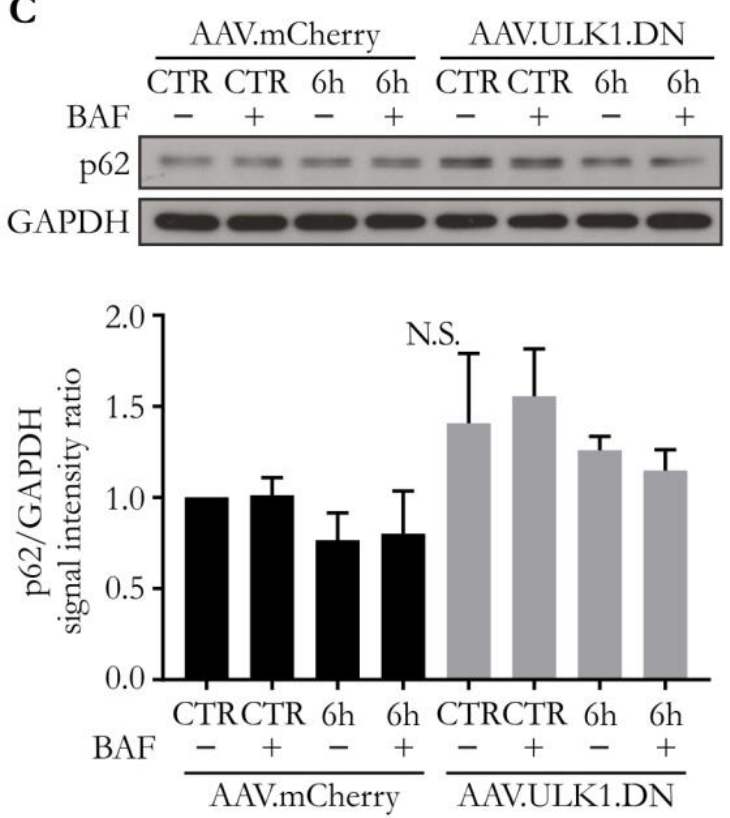

Figure 12: Autophagy markers after scratch lesion on DIV 7 and transduction with AAV.ULK1.DN. (A) Scheme of experimental setup. DOP: day of preparation of E18 rat cortical neurons. DIV: day in vitro. AAV: transduction with adeno-associated viral vectors. Bafilomycin (BAF, $10 \mathrm{nM}$ ) was added in selected conditions. (B) Representative Western blots of LC3-I/II and GAPDH (as loading control) are shown at the top. CTR: non-lesioned control. Below, quantification of band intensities normalized to GAPDH ( $\mathrm{n}=4$ independent cultures). (C) Representative Western blots of p62 and GAPDH (as loading control) are shown at the top. Below, quantification of band intensities normalized to GAPDH ( $\mathrm{n}=4$ independent cultures). Bars represent means \pm SEM. N.S.: no significant difference, according to one-way ANOVA and Sidak's multiple comparisons test.

\subsubsection{Analysis of calcium-mediated autophagy induction}

Mechanical lesions executed at several time points and after different culture durations did not result in the reproducible induction of autophagy (see 3.3.1.1, 3.3.1.2). Therefore, a different paradigm was required to mimic autophagy induction after axonal injury in vitro and 
investigate the effects of AAV.ULK1.DN on autophagic proteins. As calcium influx is a major executing step during axonal degeneration and concurred with increased autophagic levels in degenerating axons after SCI (Ribas et al. 2015), the application of the calcium ionophore A23187, leading to the pharmacological increase of intracellular calcium concentrations, was tested next.

3.3.2.1 Analysis of autophagic markers after calcium ionophore treatment on DIV 4 and transduction with AAV.ULK1.DN

After a preliminary test with different concentrations and incubation times using nontransduced rat cortical neurons (data not shown), cells were transduced with AAV.ULK1.DN and AAV.mCherry, treated with calcium ionophore $(2.5 \mu \mathrm{M})$ for $30 \mathrm{~min}$ on DIV 4 and lysed 5 h later (Figure 13A). Western blot analysis of LC3-II (Figure 13B) showed a tendency to increased levels after addition of calcium ionophore for both AAV.mCherry $(1.32 \pm 0.24$ a.u.) and AAV.ULK1.DN (1.46 \pm 0.28 a.u.), but this was far from statistical significance $(\mathrm{p}>0.43$ for both AAV vectors compared to respective controls), indicating no effect of calcium ionophore treatment on autophagy and no differences between both AAV vectors.

A

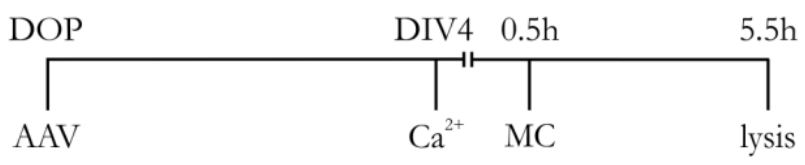

B

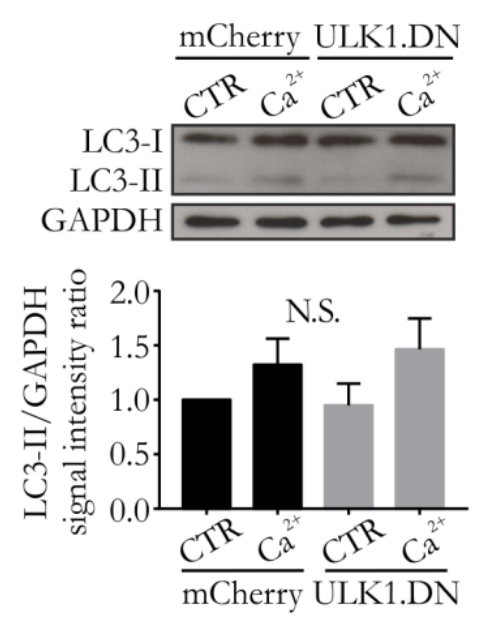

C

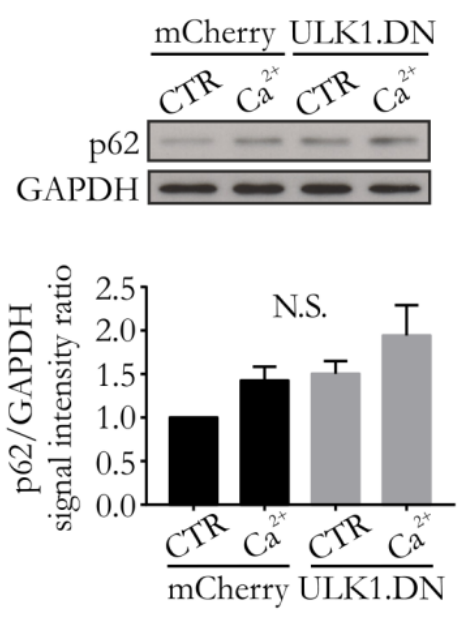

Figure 13: Autophagy markers after addition of calcium ionophore on DIV 4 and transduction with AAV.ULK1.DN. (A) Scheme of experimental setup. DOP: day of preparation of E18 rat cortical neurons. DIV: day in vitro. AAV: transduction with adeno-associated viral vectors. $\mathrm{Ca}^{2+}$ : treatment with calcium ionophore $(2.5 \mu \mathrm{M})$. MC: medium change. (B) Representative Western blots of LC3-I/II and GAPDH (as loading control) are shown at the top. CTR: untreated control. Below, quantification of band intensities normalized to GAPDH ( $\mathrm{n}=3-4$ independent cultures). (C) Representative Western blots of p62 and GAPDH (as loading control) are shown at the top. Below, quantification of band intensities normalized to 
GAPDH ( $\mathrm{n}=4$ independent cultures). Bars represent means \pm SEM. N.S.: no significant difference, according to one-way ANOVA and Sidak's multiple comparisons test.

Correspondingly, the assessment of p62 (Figure 13C) showed a tendency to increased levels after addition of calcium ionophore for cells transduced with AAV.mCherry (1.43 \pm 0.16 a.u.) and AAV.ULK1.DN (1.94 \pm 0.35 a.u.). Transduction with AAV.ULK1.DN also led to a tendency to increased p62 levels in untreated conditions $(1.50 \pm 0.15$ a.u.). Statistical analysis, however, showed that this was far from significance ( $p>0.34$ for all comparisons), corroborating that calcium ionophore treatment had no effect on autophagy.

Taken together, addition of calcium ionophore on DIV 4 did not result in a reproducible induction of autophagy. Furthermore, no difference in the levels of autophagic proteins could be detected between cells transduced with AAV.mCherry and AAV.ULK1.DN.

\subsubsection{Analysis of autophagic markers after calcium ionophore treatment on DIV 7 and transduction with AAV.ULK1.DN}

As the addition of calcium ionophore for $30 \mathrm{~min}$ on DIV 4 did not lead to autophagy induction (see 3.3.2.1), a different paradigm with longer duration of calcium ionophore administration and more mature cultures was tested next. Cortical neurons were transduced with AAV.ULK1.DN and AAV.mCherry, cultured for 7 DIV, and treated with calcium ionophore $(100 \mathrm{nM})$ for $6 \mathrm{~h}$. Additionally, cells were treated with bafilomycin to block autophagic flux in selected conditions (Figure 14A).

Western blot analysis of LC3-II (Figure 14B) showed no significant effect of calcium ionophore treatment in cells transduced with AAV.mCherry (treated: $1.48 \pm 0.19$ a.u.) or AAV.ULK1.DN (untreated: $1.61 \pm 0.09$ a.u.; treated: $1.38 \pm 0.30$ a.u.), indicating no effect of calcium ionophore treatment on autophagy. Addition of bafilomycin significantly increased LC3-II levels in cells transduced with AAV.mCherry $(5.73 \pm 1.42$ a.u.) and AAV.ULK1.DN (4.78 \pm 1.03 a.u.), but no effect of treatment with calcium ionophore was observed. Furthermore, no difference between both AAV vectors was detectable. The assessment of p62 levels (Figure 14C) showed no significant effect of treatment with calcium ionophore or bafilomycin in cells transduced with AAV.ULK1.DN and AAV.mCherry.

In summary and corresponding to the results on DIV 4, addition of calcium ionophore on DIV 7 did not result in a reproducible induction of autophagy. Furthermore, no difference in the expression of autophagic markers could be detected between cells transduced with AAV.mCherry and AAV.ULK1.DN. 
A

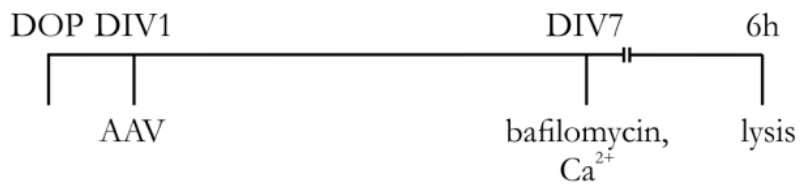

B mCherry ULK1.DN mCherry ULK1.DN

C mCherry ULK1.DN mCherry ULK1.DN

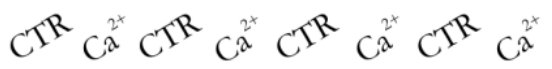
$\mathrm{BAF}-\quad-\quad-\quad+\quad+\quad+\quad+$
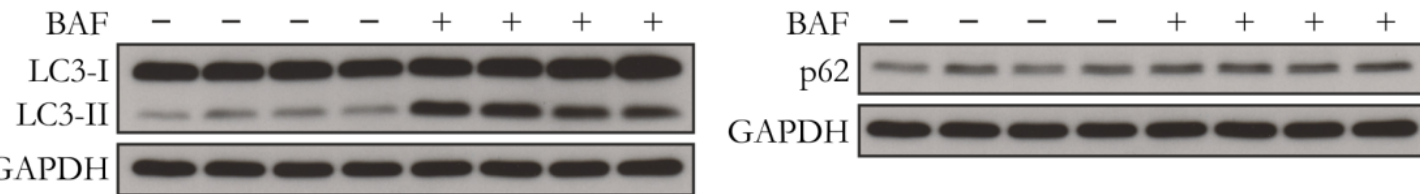
GAPDH
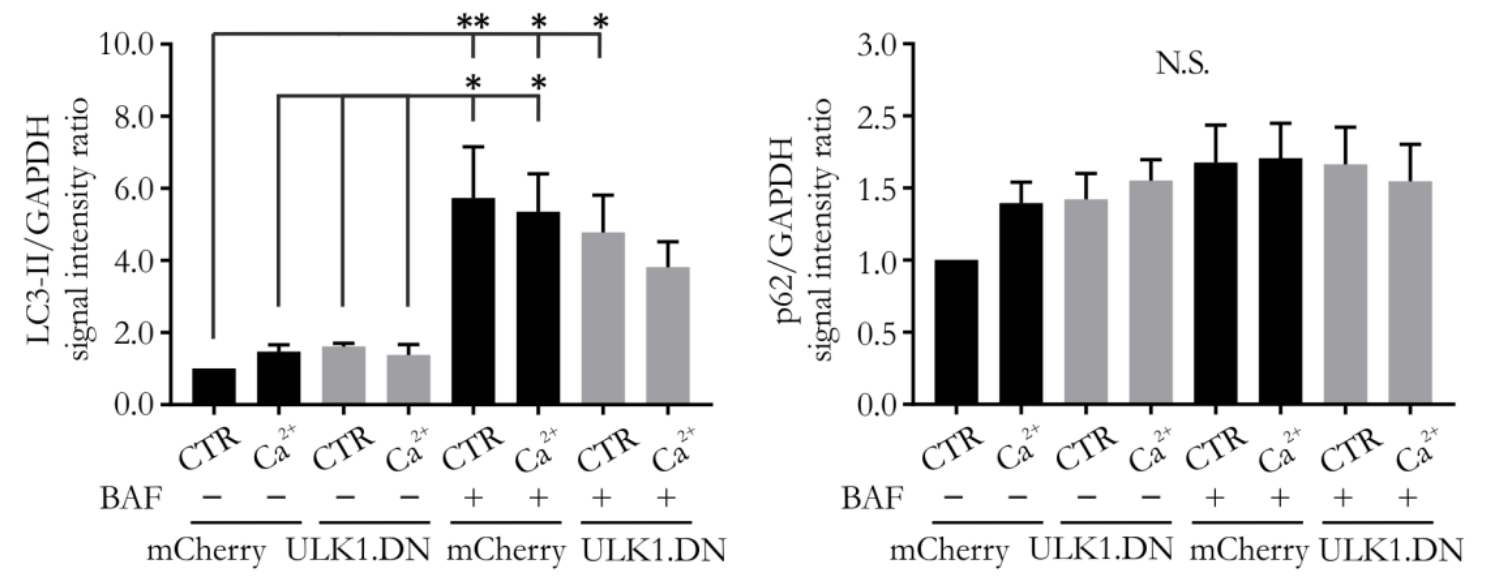

Figure 14: Autophagy markers after addition of calcium ionophore on DIV 7 and transduction with AAV.ULK1.DN. (A) Scheme of experimental setup. DOP: day of preparation of E18 rat cortical neurons. DIV: day in vitro. AAV: transduction with adeno-associated viral vectors. $\mathrm{Ca}^{2+}$ : Treatment with calcium ionophore (100 nM). Bafilomycin (BAF, $10 \mathrm{nM}$ ) was added in selected conditions. (B) Representative Western blots of LC3-I/II and GAPDH (as loading control) are shown at the top. CTR: untreated control. Below, quantification of band intensities normalized to GAPDH ( $\mathrm{n}=5$ independent cultures). (C) Representative Western blots of p62 and GAPDH (as loading control) are shown at the top. Below, quantification of band intensities normalized to GAPDH ( $\mathrm{n}=5$ independent cultures). Bars represent means \pm SEM. $* \mathrm{P}<0.05, * * \mathrm{P}$ $<0.01$, N.S.: no significant difference, according to one-way ANOVA and Sidak's multiple comparisons test.

\subsubsection{Evaluation of rapamycin-mediated autophagy induction}

Neither mechanical lesions nor treatment with calcium ionophore resulted in a detectable induction of autophagy (see 3.3.1.1, 3.3.1.2, 3.3.2.1, 3.3.2.2). As a third strategy to mimic autophagy induction after axonal injury, cells were treated with the mTOR inhibitor rapamycin in order to test pharmacological autophagy activation.

\subsubsection{Analysis of autophagic markers after rapamycin treatment on DIV 4 and transduction with AAV.ULK1.DN}

After a preliminary test with different concentrations and incubation times using nontransduced rat cortical neurons (data not shown), cortical neurons were transduced with AAV.ULK1.DN and AAV.mCherry, cultured for 4 DIV and treated with rapamycin 
$(250 \mathrm{nM})$ for $6 \mathrm{~h}$ (Figure 15A). Western blot analysis of LC3-II (Figure 15B) showed significantly higher protein expression in non-transduced cells (1.79 \pm 0.26 a.u.) and neurons transduced with AAV.mCherry (1.78 \pm 0.20 a.u.) after rapamycin administration, indicating the successful induction of autophagy. Compared to AAV.mCherry, transduction with AAV.ULK1.DN significantly reduced LC3-II levels (1.07 \pm 0.09 a.u.), suggesting ULK1.DN successfully inhibited autophagy. However, the analysis of p62 levels (Figure 15C) showed no significant differences after addition of rapamycin, neither in non-transduced cells $(0.94 \pm 0.10$ a.u. $)$ nor after transduction with AAV.mCherry $(0.86 \pm 0.13$ a.u.) and AAV.ULK1.DN (0.89 \pm 0.17 a.u.), indicating that this experimental paradigm only moderately induced autophagy.

A

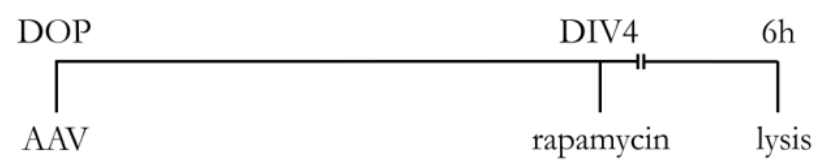

B

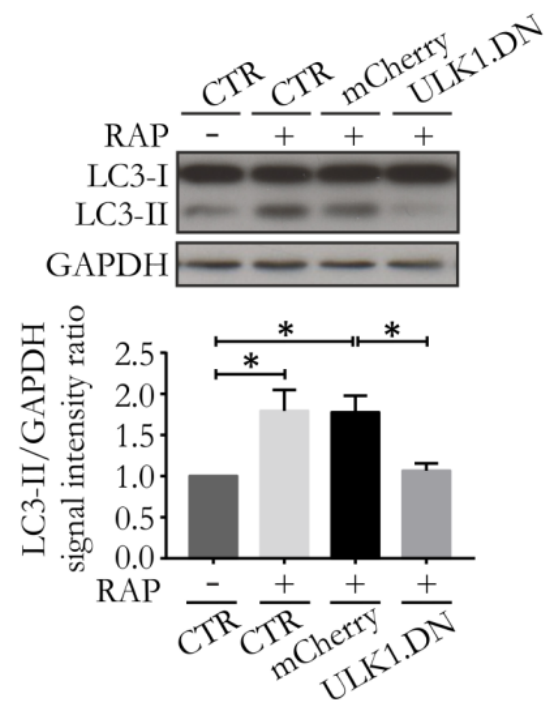

C

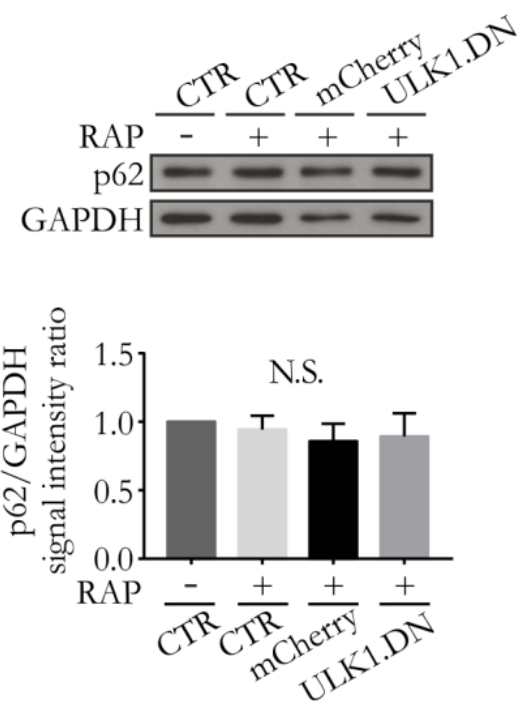

Figure 15: Autophagy induction after addition of rapamycin on DIV 4 is mildly inhibited after transduction with AAV.ULK1.DN. (A) Scheme of experimental setup. DOP: day of preparation of E18 rat cortical neurons. DIV: day in vitro. AAV: transduction with adeno-associated viral vectors. Rapamycin: addition of rapamycin (RAP, $250 \mathrm{nM}$ ) $6 \mathrm{~h}$ before lysis. (B) Representative Western blots of LC3-I/II and GAPDH (as loading control) are shown at the top. CTR: non-transduced control. Below, quantification of band intensities normalized to GAPDH ( $\mathrm{n}=4$ independent cultures). (C) Representative Western blots of p62 and GAPDH (as loading control) are shown at the top. Below, quantification of band intensities normalized to GAPDH ( $\mathrm{n}=4$ independent cultures). Bars represent means \pm SEM. $*$ P $<0.05$, N.S.: no significant difference, according to one-way ANOVA and Sidak's multiple comparisons test.

\subsubsection{Analysis of autophagic markers after rapamycin treatment on DIV 7 and} transduction with AAV.ULK1.DN

Since 6 h-treatment with rapamycin on DIV 4 only moderately induced autophagy, indicated by elevated LC3-II expression but unchanged levels of p62 (see 3.3.3.1), a different paradigm 
of rapamycin administration with higher dosage, longer incubation time, and more mature cultures was tested. Specifically, cortical neurons were transduced with AAV.ULK1.DN and AAV.mCherry, cultured for $7 \mathrm{DIV}$, and treated with rapamycin $(750 \mathrm{nM})$ for $24 \mathrm{~h}$ (Figure $16 \mathrm{~A})$.

After rapamycin administration, Western blot analysis of LC3-II levels (Figure 16B) showed significantly increased values in cells transduced with AAV.mCherry $(1.64 \pm 0.09$ a.u.) and AAV.ULK1.DN $(1.37 \pm 0.05$ a.u.) compared to their respective controls, indicating the successful induction of autophagy. In comparison with AAV.mCherry, transduction with AAV.ULK1.DN significantly decreased LC3-II levels by $~ 30 \%$ after autophagy induction with rapamycin, indicating an inhibitory effect of ULK1.DN on the autophagy cascade. The untreated control conditions showed no difference between both AAV vectors.

A

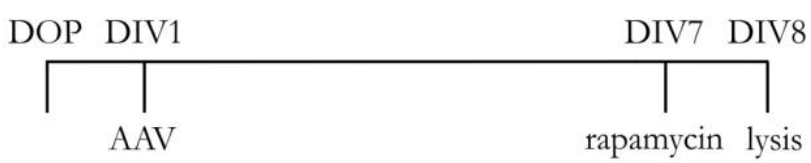

B

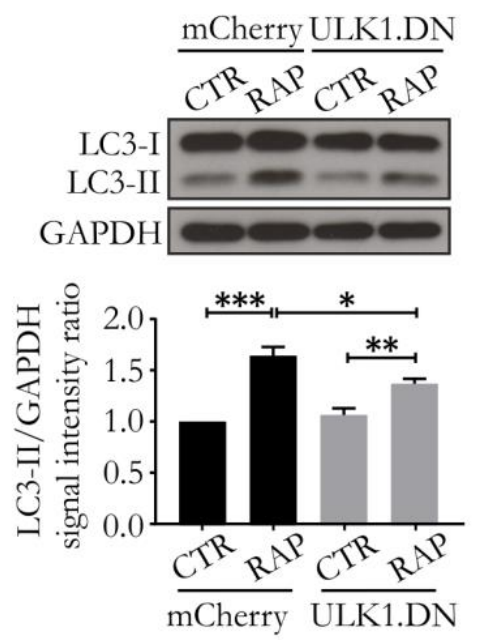

C
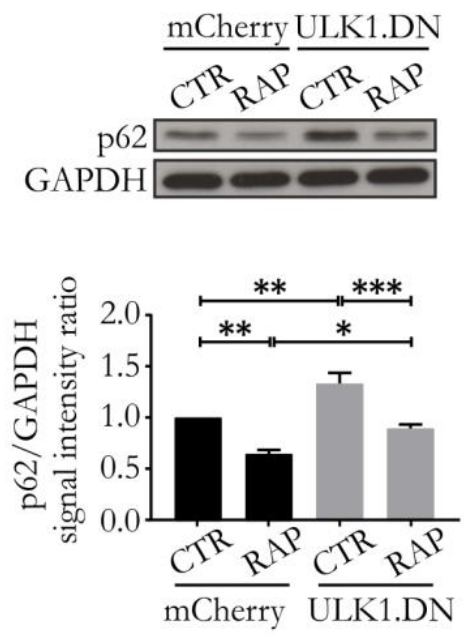

Figure 16: Autophagy induction after addition of rapamycin on DIV 7 is inhibited after transduction with AAV.ULK1.DN. (A) Scheme of experimental setup. DOP: day of preparation of E18 rat cortical neurons. DIV: day in vitro. AAV: transduction with adeno-associated viral vectors. Rapamycin: addition of rapamycin (RAP, $750 \mathrm{nM}$ ) 24 hours before lysis. (B) Representative Western blots of LC3-I/II and GAPDH (as loading control) are shown at the top. CTR: untreated control. Below, quantification of band intensities normalized to GAPDH ( $\mathrm{n}=6$ independent cultures). (C) Representative Western blots of $\mathrm{p} 62$ and GAPDH (as loading control) are shown at the top. Below, quantification of band intensities normalized to GAPDH ( $\mathrm{n}=6$ independent cultures). Bars represent means \pm SEM. $* \mathrm{P}<0.05, * * \mathrm{P}<0.01, * * * \mathrm{P}<0.001$, according to one-way ANOVA and Sidak's multiple comparisons test. Figure adapted from Vahsen et al. (2020), licensed under a CC BY 4.0 license, https://creativecommons.org/licenses/by/4.0/.

The assessment of p62 (Figure 16C) showed significantly reduced levels after treatment with rapamycin in cells transduced with AAV.mCherry $(0.65 \pm 0.04$ a.u. $)$ and AAV.ULK1.DN $(0.89 \pm 0.04$ a.u.), confirming the successful induction of autophagy in this paradigm. In 
comparison with AAV.mCherry, transduction with AAV.ULK1.DN significantly increased p62 levels by $\sim 25 \%$ in rapamycin-treated conditions, corroborating an inhibitory effect of ULK1.DN on the autophagy cascade. Furthermore, transduction with AAV.ULK1.DN led to significantly increased p62 levels in untreated cells (1.33 \pm 0.11 a.u.), as compared with AAV.mCherry.

In summary, these results demonstrate that 24 h-treatment with rapamycin $(750 \mathrm{nM})$ on DIV 7 is an effective paradigm to induce autophagy in cortical neurons in vitro. Furthermore, AAV.ULK1.DN exerts a significant inhibitory effect on autophagy induction in this model.

3.3.3.3 Analysis of additional autophagic proteins after rapamycin treatment on DIV 7 and transduction with AAV.ULK1.DN

Using the established model of rapamycin-mediated autophagy induction, by which significant inhibitory effects of AAV.ULK1.DN were demonstrated in this thesis (see 3.3.3.2), the influence of AAV.ULK1.DN on additional autophagic proteins was investigated next (Figure 17A). First, the levels of endogenous ULK1 were evaluated (Figure 17B). Western blot analysis showed no effect of treatment with rapamycin in neurons transduced with AAV.mCherry or AAV.ULK1.DN. However, in comparison with AAV.mCherry, transduction with AAV.ULK1.DN led to a significant reduction in ULK1 expression in both untreated neurons $(0.47 \pm 0.04$ a.u.) and cells treated with rapamycin (0.56 \pm 0.06 a.u.), demonstrating that ULK1.DN induces the downregulation of endogenous ULK1.

Hereafter, the expression levels of the essential autophagy mediators ATG7 and ATG5 were evaluated (Figure 17C - E). No changes in the expression of ATG7 was detectable in naïve and rapamycin-treated cells transduced with AAV.ULK1.DN and AAV.mCherry. Similarly, Western blot analysis of free ATG5 (ATG5-30) and ATG12-conjugated ATG5 (ATG5-56) showed no significant differences, suggesting that the inhibitory effect of AAV.ULK1.DN on autophagy is independent of ATG5 and ATG7.

In summary, these results demonstrate that transduction with AAV.ULK1.DN in vitro leads to the downregulation of ULK1, resulting in the inhibition of rapamycin-induced autophagy via an ATG5-ATG7-independent mechanism. 
A

DIV7 DIV8

AAV

rapamycin lysis

B

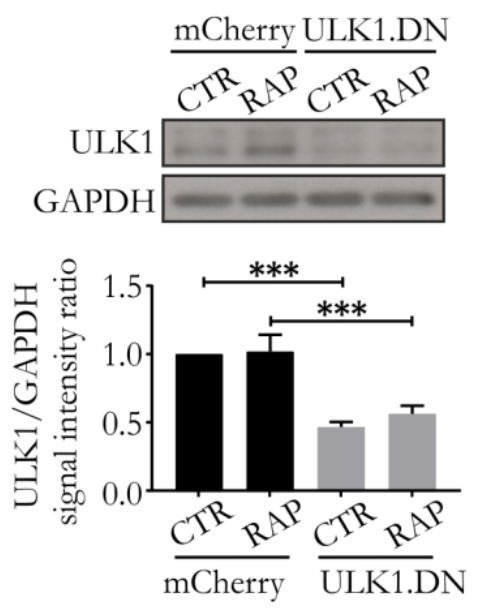

D

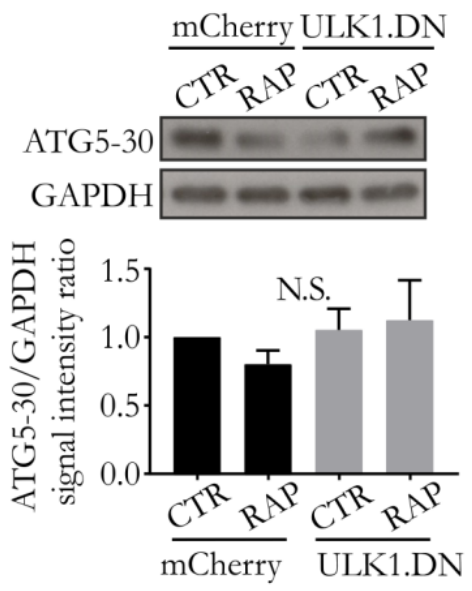

C
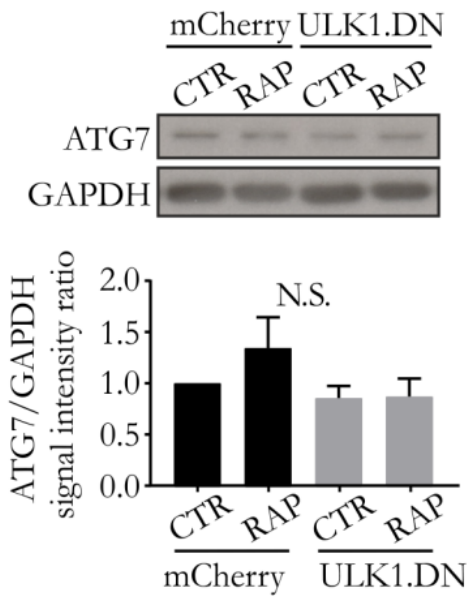

$\mathbf{E}$
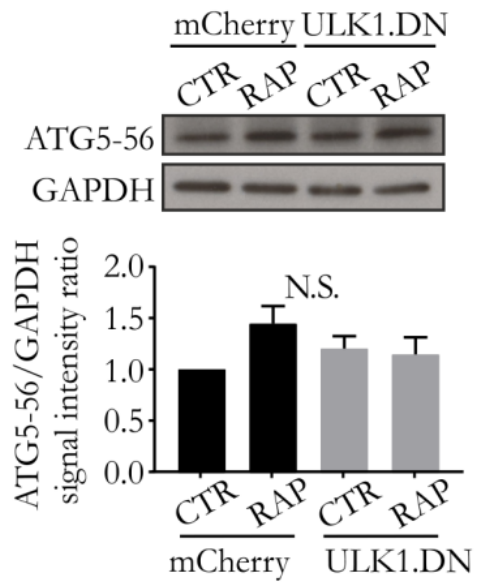

Figure 17: ULK1, ATG5, and ATG7 levels after rapamycin treatment on DIV 7 and transduction with AAV.ULK1.DN. (A) Scheme of experimental setup. DOP: day of preparation of E18 rat cortical neurons. DIV: day in vitro. AAV: transduction with adeno-associated viral vectors. Rapamycin: addition of rapamycin (RAP, $750 \mathrm{nM}) 24$ hours before lysis. (B) Representative Western blots of ULK1 and GAPDH (as loading control) are shown at the top. CTR: untreated control. Below, quantification of band intensities normalized to GAPDH ( $\mathrm{n}=6$ independent cultures). (C) Representative Western blots of ATG7 and GAPDH (as loading control) are shown at the top. Below, quantification of band intensities normalized to GAPDH ( $\mathrm{n}=6$ independent cultures). (D) Representative Western blots of free ATG5 (30 kDa) and GAPDH (as loading control) are shown at the top. Below, quantification of band intensities normalized to GAPDH ( $\mathrm{n}=4-5$ independent cultures). (E) Representative Western blots of ATG12-conjugated ATG5 (56 kDa) and GAPDH (as loading control) are shown at the top. Below, quantification of band intensities normalized to GAPDH ( $\mathrm{n}=4-5$ independent cultures). Bars represent means \pm SEM. $* * * \mathrm{P}<0.001$, N.S.: no significant difference, according to one-way ANOVA and Sidak's multiple comparisons test. Figure adapted from Vahsen et al. (2020), licensed under a CC BY 4.0 license, https://creativecommons.org/licenses/by/4.0/. 


\subsection{Transduction with AAV.ULK1.DN does not affect cell survival}

Due to its crucial role in cell homeostasis, the autophagy cascade is tightly connected to cell survival pathways. Having demonstrated an inhibitory effect of transduction with AAV.ULK1.DN on rapamycin-induced autophagy (see 3.3.3.2), one could speculate that this inhibition of autophagy might have an influence on cell survival. To assess this, cortical neurons were transduced with AAV.ULK1.DN and AAV.mCherry, cultured for 7 DIV, and treated with rapamycin for $24 \mathrm{~h}$ (Figure 18A).

Western blot analysis of cleaved caspase 3, a widely used marker of apoptotic cell death, was then performed to assess cell survival. No statistically significant difference in cleaved caspase 3 levels could be observed between AAV.ULK1.DN and AAV.mCherry, neither in untreated conditions nor after rapamycin treatment (Figure 18B), indicating no effect of AAV.ULK1.DN on apoptosis in naïve and rapamycin-treated conditions.

A

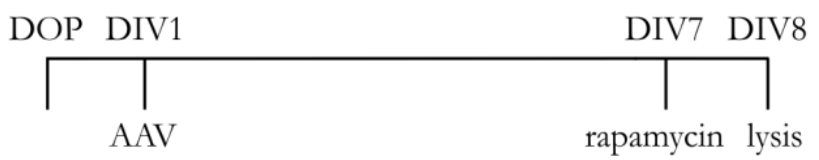

\section{B}
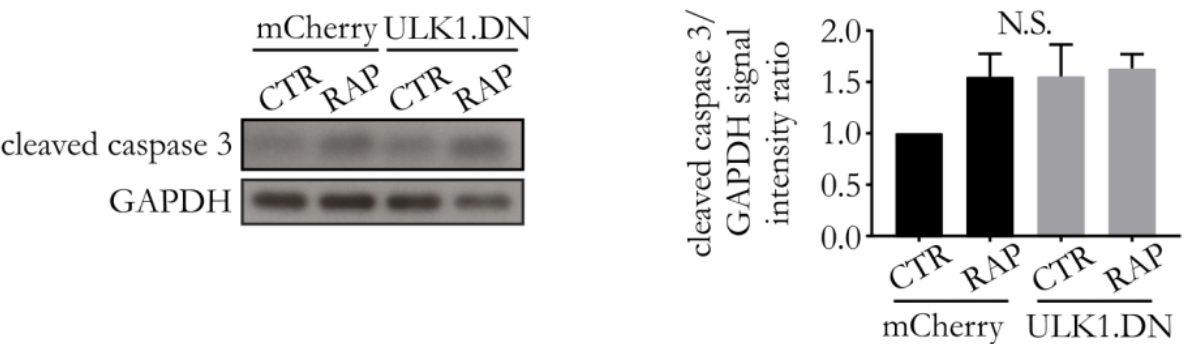

Figure 18: Cleaved caspase 3 levels remain unchanged after rapamycin treatment and transduction with AAV.ULK1.DN. (A) Scheme of experimental setup for the evaluation of cell survival. DOP: day of preparation of E18 rat cortical neurons. DIV: day in vitro. AAV: transduction with adeno-associated viral vectors. Rapamycin: addition of rapamycin (RAP, $750 \mathrm{nM}$ ) 24 hours before lysis. (B) A representative Western blot of cleaved caspase 3 is shown on the left, the quantification of band intensities normalized to GAPDH as loading control is depicted on the right ( $\mathrm{n}=6$ independent cultures). CTR: untreated control. Bars represent means \pm SEM. N.S.: no significant difference, according to one-way ANOVA and Sidak's multiple comparisons test. Figure adapted from Vahsen et al. (2020), licensed under a CC BY 4.0 license, https://creativecommons.org/licenses/by/4.0/.

Furthermore, in an additional experimental paradigm, apoptosis was actively induced in transduced neurons to evaluate whether a discrete effect of AAV.ULK1.DN on cell survival might be unmasked under stress conditions. Specifically, cortical neurons transduced with AAV.mCherry and AAV.ULK1.DN were treated on DIV 7 with increasing dosages of staurosporine, a widely employed inductor of cell death. After a $24 \mathrm{~h}$-incubation, samples of the cell culture medium were used for the analysis of cytotoxicity with the ToxiLight ${ }^{\mathrm{TM}}$ assay (Figure 19A). 
A linear trend to increasing toxicity with rising staurosporine dosages could be detected in cells transduced with AAV.mCherry $(30 \mathrm{nM}: 1.4 \pm 0.0$ a.u.; $100 \mathrm{nM:} 1.9 \pm 0.4$ a.u.; 300 nM: $2.7 \pm 0.5$ a.u.) and AAV.ULK1.DN (untreated: $1.0 \pm 0.0$ a.u.; 30 nM: $1.4 \pm 0.1$ a.u.; $100 \mathrm{nM}: 1.7 \pm 0.1$ a.u.; $300 \mathrm{nM}: 3.0 \pm 0.6$ a.u.), which reached statistical significance for $300 \mathrm{nM}$ staurosporine, compared to the respective untreated controls (Figure 19B). However, no significant differences between both AAV vectors were detectable, confirming the absence of an effect of AAV.ULK1.DN on cell survival also after apoptosis induction.

A

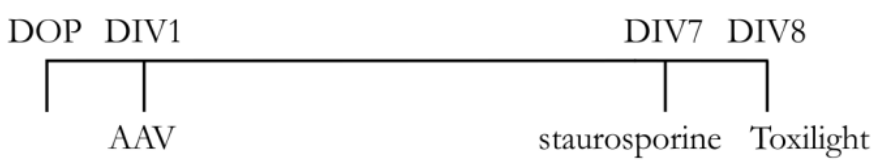

B

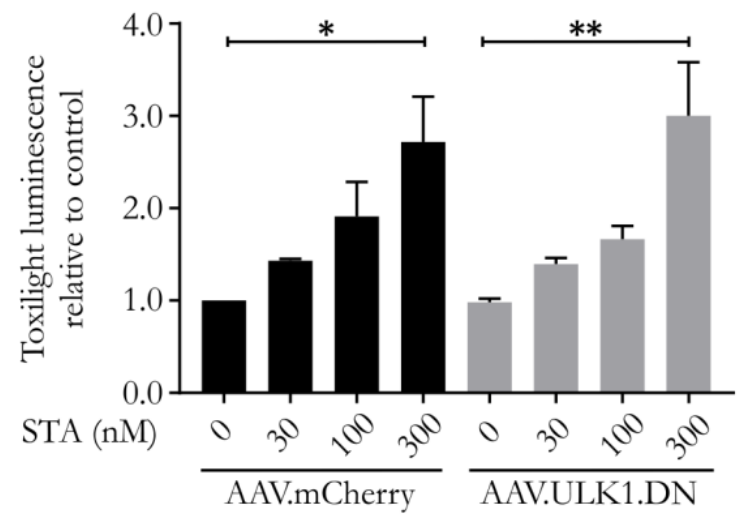

Figure 19: Evaluation of cell survival after staurosporine-mediated apoptosis induction and transduction with AAV.ULK1.DN. (A) Scheme of experimental setup for the evaluation of cell survival. DOP: day of preparation of E18 rat cortical neurons. DIV: day in vitro. AAV: transduction with adenoassociated viral vectors. Staurosporine (STA): addition of staurosporine (30 nM, $100 \mathrm{nM}, 300 \mathrm{nM}) 24$ hours before ToxiLight ${ }^{\mathrm{TM}}$ cytotoxicity assay. (B) Luminescence after addition of ToxiLight ${ }^{\mathrm{TM}}$ reagents quantifies cell death after treatment with different concentrations $(30 \mathrm{nM}, 100 \mathrm{nM}, 300 \mathrm{nM})$ of the apoptosis-inducing agent staurosporine ( $\mathrm{n}=3$ independent cultures). Bars represent means \pm SEM. $* \mathrm{P}<0.05, * * \mathrm{P}<0.01$, according to one-way ANOVA and Sidak's multiple comparisons test. Figure adapted from Vahsen et al. (2020), licensed under a CC BY 4.0 license, https://creativecommons.org/licenses/by/4.0/.

\subsection{AAV.ULK1.DN protects against acute axonal degeneration after axotomy in vitro}

A previous study in the laboratory (Ribas et al. 2015) had demonstrated an early and strong post-SCI upregulation of autophagic proteins, most prominently ULK1, raising the question of whether ULK1-dependent autophagy induction might represent an important executing mechanism in axonal degeneration. Having demonstrated a significant inhibition of rapamycin-mediated autophagy induction by AAV.ULK1.DN (see 3.3.3.2), it was hypothesized that AAV.ULK1.DN might have beneficial effects on AAD. To assess this, 
cortical neurons were seeded into microfluidic culture platforms, which allow for the performance of selective axonal lesions in vitro, and transduced with AAV.ULK1.DN or control. Seven days later, axons were axotomized, and live-imaging was performed over $6 \mathrm{~h}$ to investigate AAD (Figure 20A).

A

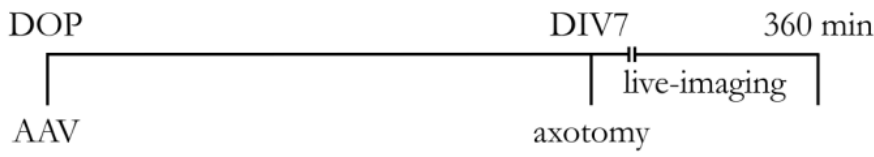

B

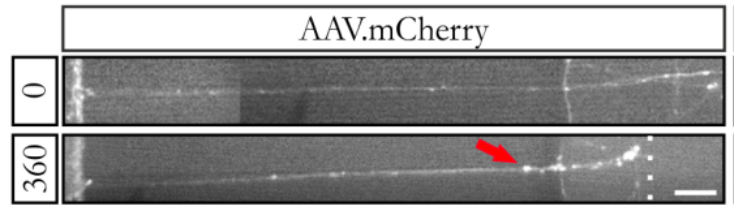

C

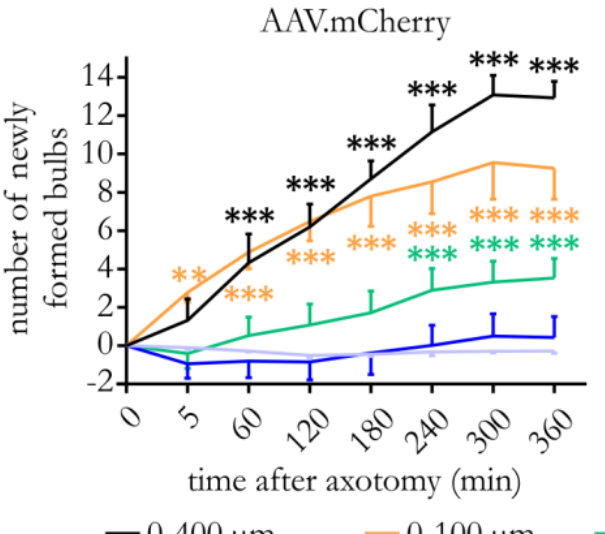

\begin{tabular}{|c|}
\hline AAV.ULK1.DN \\
\hline \hline$\ldots$ \\
\hline
\end{tabular}

D
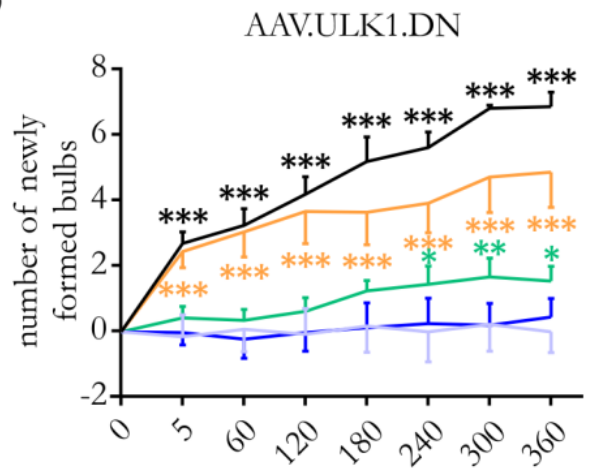

time after axotomy ( $\mathrm{min})$

$$
-0-400 \mu \mathrm{m} \quad-0-100 \mu \mathrm{m} \quad-100-200 \mu \mathrm{m} \quad-200-300 \mu \mathrm{m} \quad-300-400 \mu \mathrm{m}
$$

E

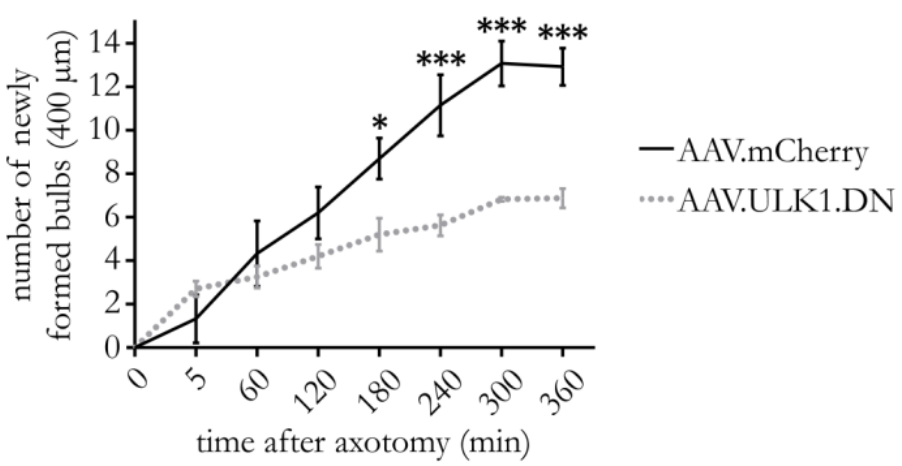

Figure 20: AAV.ULK1.DN attenuates AAD after selective axonal lesion in vitro. (A) Scheme of experimental setup. DOP: day of preparation of E18 rat cortical neurons and seeding into microfluidic culture platforms. DIV: day in vitro. AAV: transduction with adeno-associated viral vectors. (B) Representative images of axons growing in microfluidic culture platforms transduced with given AAV vectors. Exemplary photomicrographs were taken directly before and 360 min after axotomy. Red arrow: example of axonal bulb used for quantification. Dotted line: lesion site. Scale bar: $50 \mu \mathrm{m}$. (C, D) Detailed quantifications of the number of newly formed bulbs within different distances (indicated by colors) proximal to the area of lesion at the indicated time points after axotomy and transduction with AAV.mCherry $(\mathbf{C}, \mathrm{n}=4$ independent cultures) or AAV.ULK1.DN (D, n $=4$ independent cultures). (E) Number of newly formed bulbs within $400 \mu \mathrm{m}$ distance proximal to the lesion at the indicated time points after axotomy in cells transduced with AAV.mCherry and AAV.ULK1.DN ( $\mathrm{n}=4$ independent cultures). Bars represent means \pm SEM. $* \mathrm{P}<0.05,{ }^{* * \mathrm{P}}<0.01,{ }^{* * *} \mathrm{P}<$ 0.001, according to two-way RM ANOVA and Dunnett's $(\mathbf{C}, \mathbf{D})$ or Sidak's multiple comparison's test $(\mathbf{E})$. 
Figure adapted from Vahsen et al. (2020), licensed under a CC BY 4.0 license, https://creativecommons.org/licenses/by/4.0/.

Over the course of the $6 \mathrm{~h}$ after lesion, axonal bulbs, an early hallmark of AAD, became visible in lesioned axons transduced with both AAV vectors (Figure 20B). Quantification of the number of newly formed axonal bulbs within the $400 \mu \mathrm{m}$ section proximal to the lesion site, which corresponds to the axonal area affected by AAD in vivo, showed a significant timedependent increase after transduction with both AAV.mCherry and AAV.ULK1.DN (Figure 20C, D). Interestingly, these bulbs mainly accumulated in the areas $0-100 \mu \mathrm{m}$ and $100-200 \mu \mathrm{m}$ proximal to the lesion. Compared with AAV.mCherry, transduction with AAV.ULK1.DN significantly delayed the formation of new bulbs within the $400 \mu \mathrm{m}$ section proximal to the lesion site starting at 180 min after axotomy (Figure 20E). 360 min after axotomy, the number of newly formed bulbs was $\sim 50 \%$ lower in cells transduced with AAV.ULK1.DN (6.88 \pm 0.45 a.u.) when compared to AAV.mCherry (12.93 \pm 0.86 a.u.).

Taken together, these results therefore indicate that AAV.ULK1.DN attenuates AAD up to $6 \mathrm{~h}$ after axonal injury in vitro.

\subsection{Axonal regeneration after axotomy in vitro is enhanced by AAV.ULK1.DN}

The attenuation of AAD observed in neurons transduced with AAV.ULK1.DN in vitro for up to $6 \mathrm{~h}$ after axotomy (see 3.5) raised the question of whether AAV.ULK1.DN might also exert beneficial effects at longer time points after injury and potentially also enhance axonal regeneration. To assess this, cortical neurons were again cultured in microfluidic chambers and transduced with AAV.mCherry and AAV.ULK1.DN. After selective axonal lesions, axonal re-growth was evaluated by live-imaging over the $96 \mathrm{~h}$ after the axotomy (Figure 21A).

Regenerative outgrowth of axons after lesion could be seen in neurons transduced with both AAV.mCherry and AAV.ULK1.DN (Figure 21B). To analyze this quantitatively, the relative number of regenerating axons (the number of re-growing axons at a given time point divided by the number of axons before axotomy) was counted at defined distances $(100-1000 \mu \mathrm{m})$ from the exit of the microgrooves for each time point $(24-96 \mathrm{~h}) .24 \mathrm{~h}$ after axotomy, transduction with AAV.ULK1.DN significantly increased the relative number of regenerating axons at $100 \mu \mathrm{m}(0.47 \pm 0.08$ a.u. $)$ and $200 \mu \mathrm{m}$ distance $(0.24 \pm 0.06$ a.u. $)$ when compared with AAV.mCherry $(100 \mu \mathrm{m}: 0.20 \pm 0.05$ a.u.; $200 \mu \mathrm{m}: 0.04 \pm 0.03$ a.u.) (Figure 21C). $48 \mathrm{~h}$ post-injury, significantly higher values could be quantified after transduction with AAV.ULK1.DN at $100 \mu \mathrm{m}$ (1.05 \pm 0.16 a.u.), $200 \mu \mathrm{m}$ (0.74 \pm 0.12 a.u.) and $400 \mu \mathrm{m}$ distance 
(0.26 \pm 0.08 a.u. $)$ when compared to AAV.mCherry $(100 \mu \mathrm{m}: \quad 0.39 \pm 0.12$ a.u.; $200 \mu \mathrm{m}: 0.10 \pm 0.07$ a.u.; $400 \mu \mathrm{m}: 0.00 \pm 0.00$ a.u.) (Figure 21D).

A

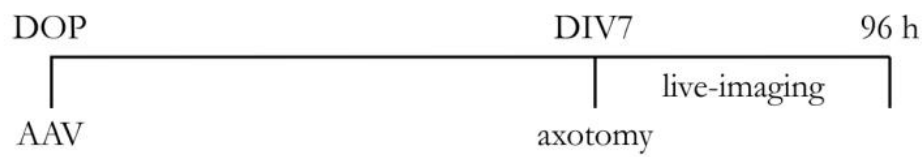

B

B AAV.mCherry $\quad$ AAV.ULK1.DN

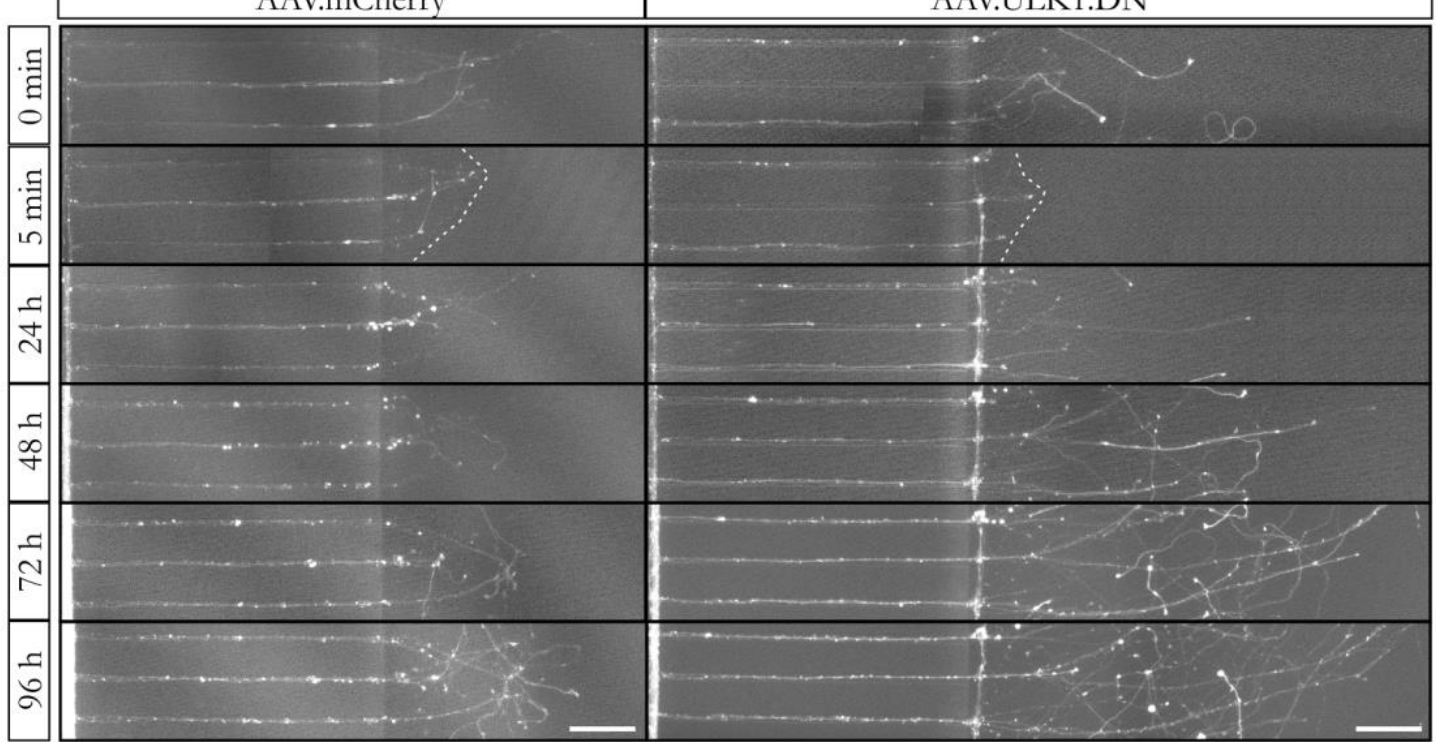

C

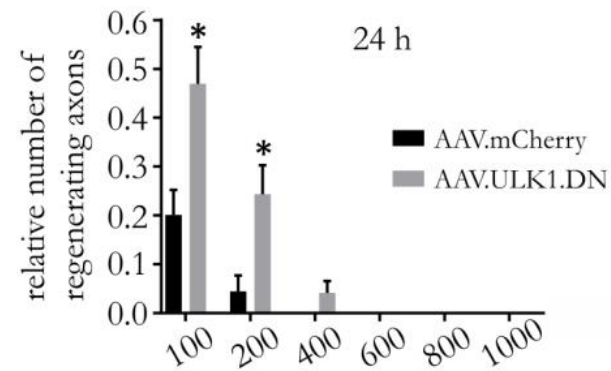

distance from exit $[\mu \mathrm{m}]$

E

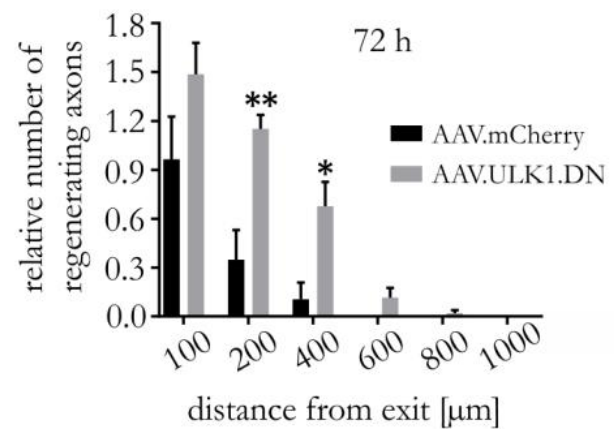

D

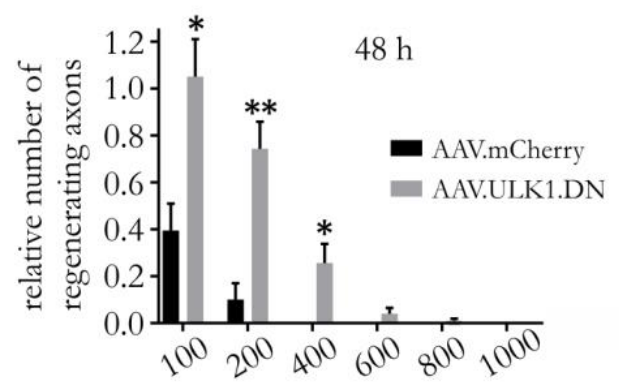

distance from exit $[\mu \mathrm{m}]$

F

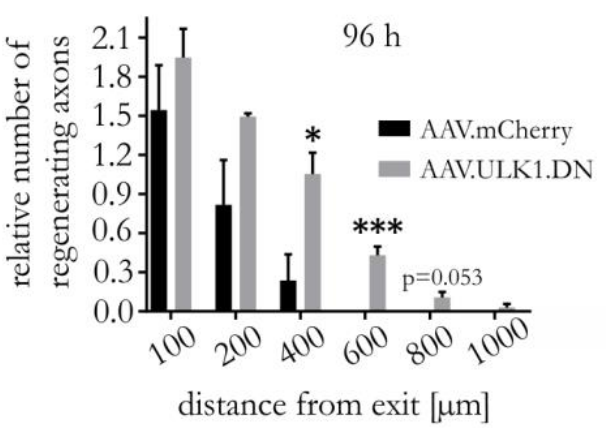

Figure 21: AAV.ULK1.DN fosters axonal regeneration after axotomy in vitro. (A) Scheme of experimental setup. DOP: day of preparation of E18 rat cortical neurons and seeding into microfluidic culture platforms. DIV: day in vitro. AAV: transduction with adeno-associated viral vectors. (B) Representative images of axons growing in microfluidic culture platforms after transduction with viral vectors. Photos were taken directly before $(0 \mathrm{~min})$ and after axotomy $(5 \mathrm{~min})$, and then in $24 \mathrm{~h}$-intervals. For the $5 \mathrm{~min}$ and $24 \mathrm{~h}$ time points of AAV.ULK1.DN, the blank area distal to the longest axon was filled with grey color for visualization purposes. White dashed line: area of lesion. Scale bar: $100 \mu \mathrm{m}$. (C - F) Quantifications of the relative number of regenerating axons after transduction with AAV.mCherry and AAV.ULK1.DN at the indicated distances 
from the microgroove exit at $24-96 \mathrm{~h}$ after axotomy ( $\mathrm{n}=4$ independent cultures). Bars represent means \pm $\mathrm{SEM} ; * \mathrm{P}<0.05,{ }^{* *} \mathrm{P}<0.01,{ }^{* * *} \mathrm{P}<0.001$, according to two-tailed unpaired t-test for each distance. Figure

adapted from Ribas et al. (2021), licensed under a CC BY 4.0 license, https://creativecommons.org/licenses/by/4.0/.

$72 \mathrm{~h}$ after axotomy, a significant difference in the relative number of regenerating axons was detectable in cells transduced with AAV.ULK1.DN at $200 \mu \mathrm{m}(1.15 \pm 0.09$ a.u.) and $400 \mu \mathrm{m}$ distance $(0.68 \pm 0.15$ a.u. $), \quad$ compared to AAV.mCherry $(200 \mu \mathrm{m}: 0.35 \pm 0.18$ a.u.; $400 \mu \mathrm{m}: 0.10 \pm 0.10$ a.u.) (Figure $21 \mathrm{E}) .96 \mathrm{~h}$ post-injury, significantly higher values were still visible after transduction with AAV.ULK1.DN at $400 \mu \mathrm{m}(1.05 \pm 0.17$ a.u.) and $600 \mu \mathrm{m}$ distance $(0.43 \pm 0.07$ a.u. $)$ as compared to AAV.mCherry $(400 \mu \mathrm{m}: 0.24 \pm 0.20$ a.u., $600 \mu \mathrm{m}: 0.00 \pm 0.00$ a.u.) (Figure 21F). Moreover, AAV.mCherry-transduced axons did not grow beyond $400 \mu \mathrm{m}$, whereas axons transduced with AAV.ULK1.DN reached up to $1000 \mu \mathrm{m}$ in length.

In summary, these data demonstrate a pro-regenerative effect of transduction with AAV.ULK1.DN up to $96 \mathrm{~h}$ post-injury in vitro.

\subsection{AAV.ULK1.DN promotes neurite outgrowth on permissive and growth-inhibitory substrate in vitro}

Thus far, this thesis has demonstrated that AAV.ULK1.DN enhances the intrinsic capacity of axons to regenerate after axonal injury in neuronal mono-cultures (see 3.6). After lesion in the adult CNS, however, axons are not only hampered by low intrinsic growth potential but are also exposed to extrinsic inhibitory signals presented by glial cells and the extracellular matrix. To assess whether transduction with AAV.ULK1.DN might also counteract these inhibitory environmental cues, cortical neurons were transduced with AAV.mCherry and AAV.ULK1.DN and grown on the permissive substrate laminin as well as the growthinhibiting matrix CSPG (Figure 22A). After 7 days in culture, live-imaging was performed to analyze neurite outgrowth (Figure 22B).

Cortical neurons transduced with AAV.mCherry had a mean absolute neurite length per cell of $564.6 \pm 85.9 \mu \mathrm{m}$ on the permissive substrate laminin, while transduction with AAV.ULK1.DN led to an increase to $729.3 \pm 103.7 \mu \mathrm{m}$ (data not shown). To account for variability in the absolute neurite length between independent cultures, the relative neurite length per cell (the absolute neurite length per cell for each condition normalized to the absolute neurite length of the control condition, i.e. AAV.mCherry-transduced neurons on laminin) was calculated. Compared to neurons transduced with AAV.mCherry and grown on laminin, plating on the non-permissive substrate CSPG led to a significant reduction in 
the mean relative neurite length per cell $(74.01 \pm 2.78 \%$ ) (Figure $22 \mathrm{C})$. Compared with AAV.mCherry, transduction with AAV.ULK1.DN significantly increased the mean relative neurite length on laminin $(129.60 \pm 1.46 \%)$. Furthermore, cortical neurons transduced with AAV.ULK1.DN showed significant 1.5-fold longer neurites on CSPG $(110.90 \pm 9.00 \%)$ as compared to AAV.mCherry.

Taken together, these data show significantly enhanced neurite outgrowth on both permissive and non-permissive substrate in vitro, demonstrating that AAV.ULK1.DN also counteracts inhibitory environmental signaling.
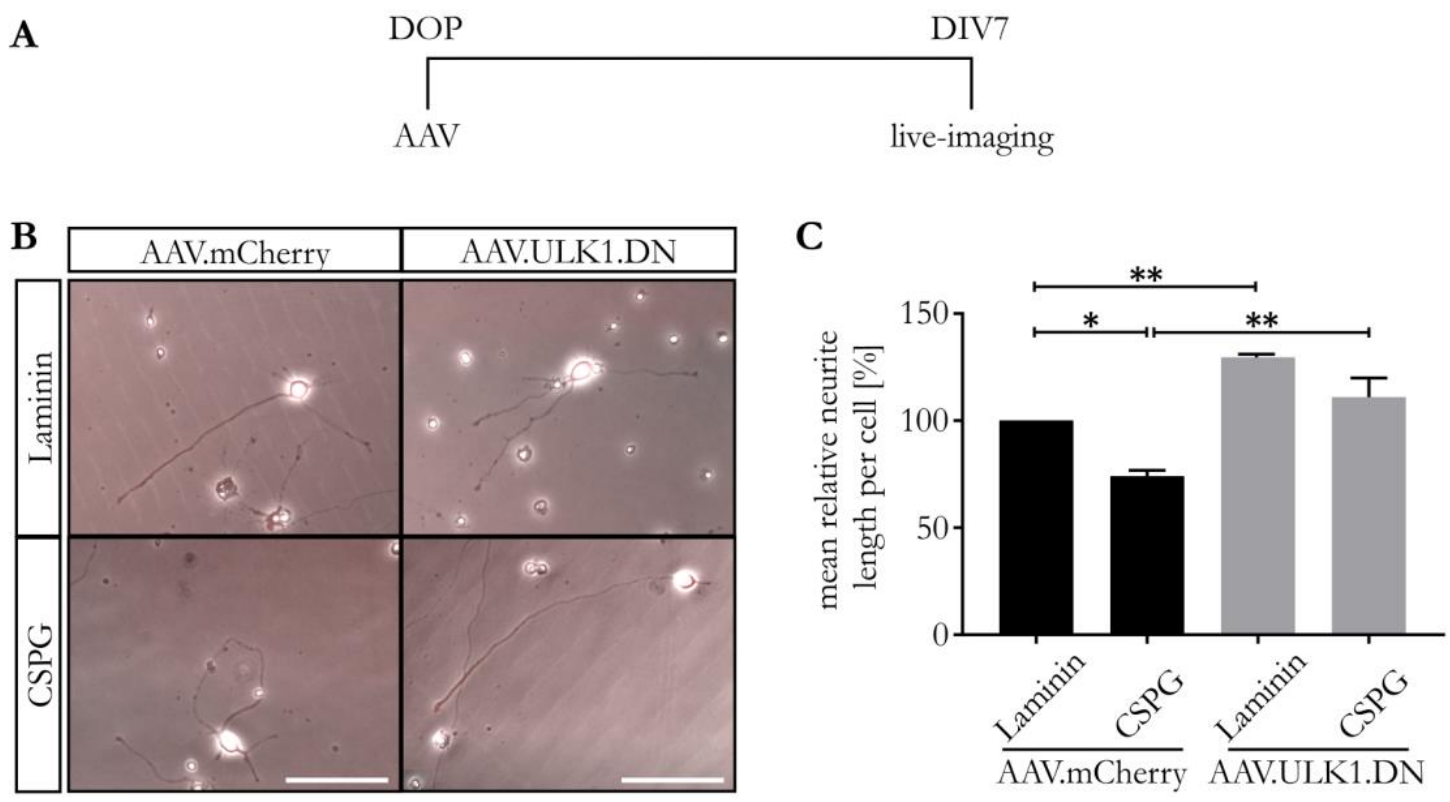

Figure 22: AAV.ULK1.DN enhances neurite outgrowth of rat cortical neurons cultured on permissive and non-permissive substrate in vitro. (A) Scheme of experimental setup. DOP: day of preparation of E18 rat cortical neurons. DIV: day in vitro. AAV: transduction with adeno-associated viral vectors. (B) Representative images of cortical neurons cultured on permissive (laminin) and non-permissive substrate (CSPG) and transduced with viral vectors. Scale bar: $100 \mu \mathrm{m}$. (C) Quantification of the mean relative neurite length per cell (the absolute neurite length per cell for each condition normalized to the absolute neurite length of the control condition, i.e. AAV.mCherry-transduced neurons on laminin) after transduction with AAV.mCherry and AAV.ULK1.DN ( $\mathrm{n}=3$ independent cultures). Bars represent means $\pm \mathrm{SEM} ; * \mathrm{P}<0.05$, $* * \mathrm{P}<0.01$, according to one-way ANOVA and Sidak's multiple comparisons test. Figure adapted from Ribas et al. (2021), licensed under a CC BY 4.0 license, https://creativecommons.org/licenses/by/4.0/. 


\subsection{Molecular mechanisms of axon protection and regeneration - proteomic analysis of cortical neurons transduced with AAV.ULK1.DN}

\subsubsection{Proteomic changes induced by AAV.ULK1.DN}

The beneficial effects observed after the transduction of cultured cortical neurons with AAV.ULK1.DN, namely an attenuation of AAD, enhanced axonal regeneration, and increased neurite outgrowth on permissive and non-permissive substrate, sparked interest in a detailed analysis of the underlying molecular mechanisms. In this thesis, a significant inhibition of the autophagy cascade by AAV.ULK1.DN was already demonstrated (see 3.3.3.2). Autophagy inhibition was shown to be protective from lesion-induced axonal degeneration in vivo in previous studies in the laboratory (see 1.2.1) and thus seemed likely to be one molecular means mediating the positive effects of AAV.ULK1.DN. However, considering the numerous interactions between ULK1 and various other proteins (see 1.4), it appeared possible that AAV.ULK1.DN might potentially exert its beneficial effects via the regulation of further molecular mechanisms in addition to autophagy. Therefore, to investigate the regulation of target proteins by AAV.ULK1.DN in an unbiased approach, a proteomic analysis was performed. Rat cortical neurons transduced with AAV.mCherry and AAV.ULK1.DN were cultured for $8 \mathrm{DIV}$ and then subjected to quantitative proteomic profiling. Out of 1988 quantifiable proteins, 122 proteins were significantly regulated by AAV.ULK1.DN when compared with AAV.mCherry (Figure 23).

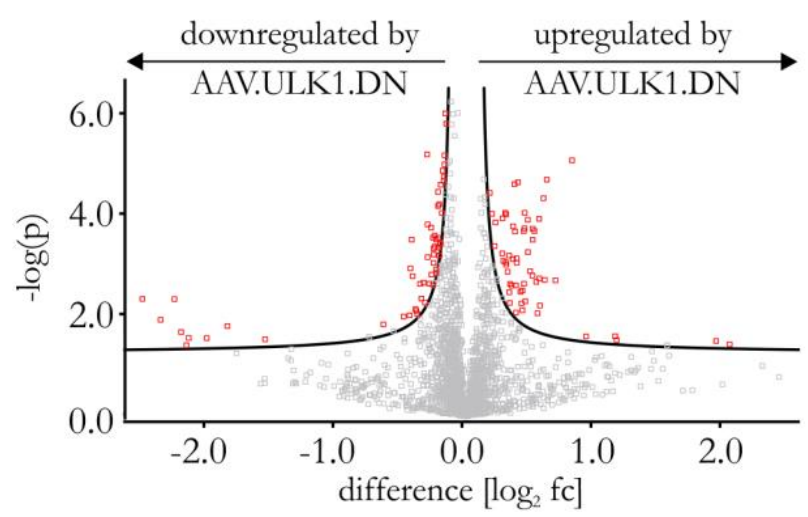

Figure 23: Quantitative proteomic profiling reveals 122 significantly regulated proteins after transduction with AAV.ULK1.DN. Lysates obtained from E18 rat cortical neurons on DIV 8 after transduction with AAV.mCherry or AAV.ULK1.DN were subjected to quantitative proteomic profiling. The volcano plot shows all 1988 quantifiable proteins in SWATH-mass spectrometry, 122 significantly regulated proteins according to two-sided t-test with permutation-based FDR assessment (FDR $=0.1, \mathrm{~s} 0=0.05$ ) appear in red ( $\mathrm{n}=2$ independent cultures, three technical replicates for each condition for each experiment). Figure adapted from Vahsen et al. (2020), licensed under a CC BY 4.0 license, https://creativecommons.org/licenses/by/4.0/. 
For validation purposes, Western blots against selected proteins with significant regulation in the proteomic analysis were performed using additional independent cultures. The list of the ten proteins with strongest up- or downregulation in proteomics (Figure 24A) showed two particularly interesting candidates because of their roles in processes implicated in axonal degeneration or neurite outgrowth: p21-activated kinase 2 (PAK2) and thousand and one amino acid protein kinase 1 (TAOK1). Corresponding to the result of the proteomic analysis $\left(\log _{2}\right.$ fc: -2.17), Western blot analysis of PAK2 showed a significant decrease in neurons transduced with AAV.ULK1.DN (0.78 \pm 0.06 a.u.) when compared with AAV.mCherry (Figure 24B). Similarly, a Western blot against TAOK1 showed significantly lower levels after transduction with AAV.ULK1.DN (0.85 \pm 0.03 a.u.) (Figure 24C), confirming the result found in the proteomic analysis $\left(\log _{2}\right.$ fc: -1.85$)$.
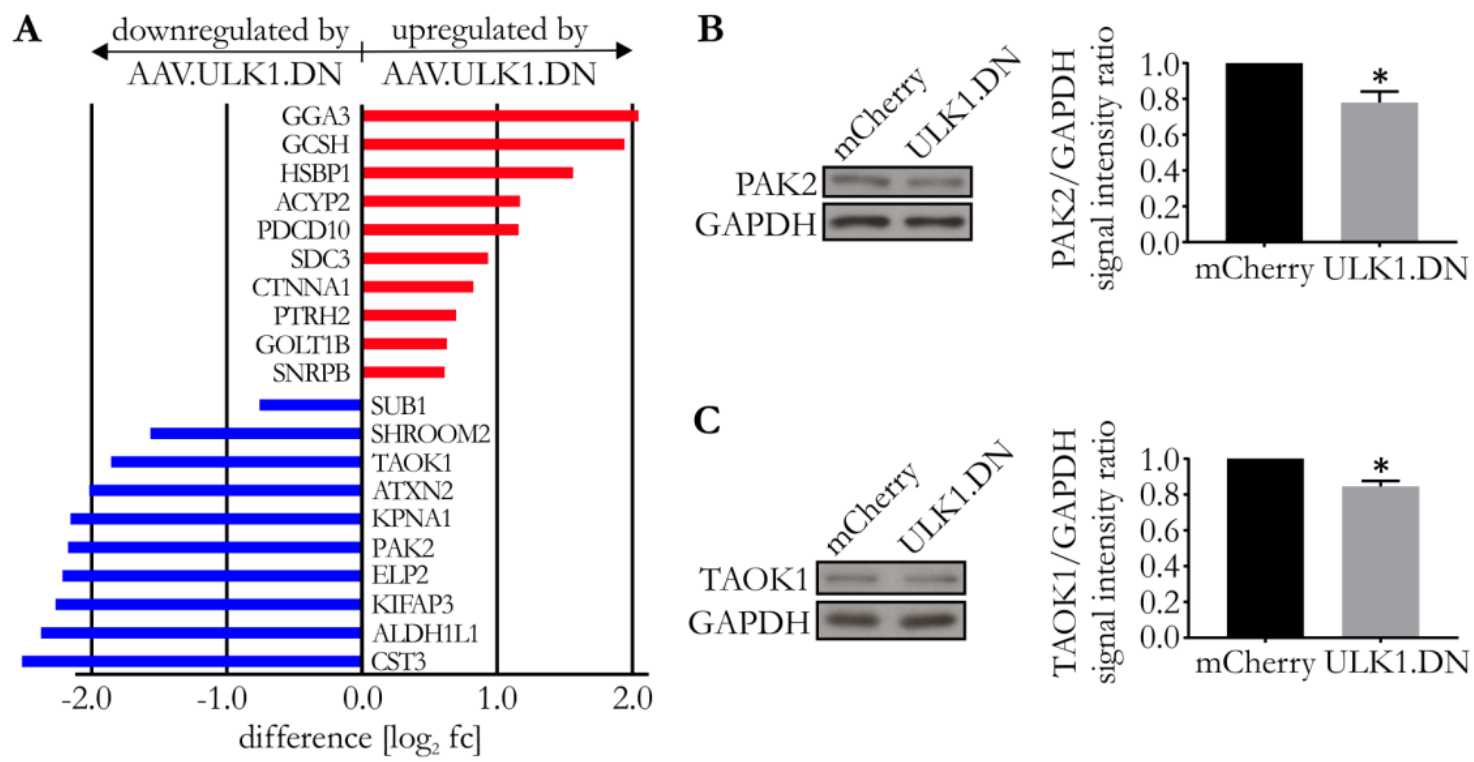

C

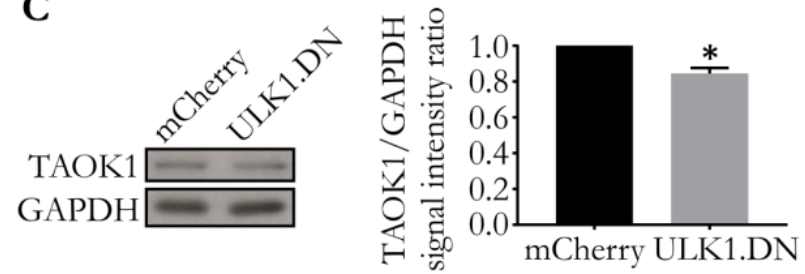

Figure 24: Overview of proteins with strongest up- and downregulation in quantitative proteomic profiling after transduction with AAV.ULK1.DN and Western blot validation of two regulated targets. Lysates obtained from E18 rat cortical neurons on DIV 8 after transduction with AAV.mCherry or AAV.ULK1.DN were subjected to proteomic analysis. (A) Overview of the ten proteins with strongest upand downregulation. (B, C) Western blot analysis against PAK2 and TAOK1 was performed to validate the results. Representative Western blots of PAK2 and TAOK1 are shown on the left, the quantifications of band intensities normalized to GAPDH (as loading control) are depicted on the right ( $\mathrm{n}=4-5$ independent cultures different to those used for the proteomic analysis). Bars represent means \pm SEM. $* \mathrm{P}<0.05$ according to onesample t-test. Figure adapted from Vahsen et al. (2020), licensed under a CC BY 4.0 license, https://creativecommons.org/licenses/by/4.0/.

\subsubsection{A prominent regulation of proteins associated with splicing and translation is mediated by AAV.ULK1.DN}

To investigate the biological relevance and function of the proteins regulated by AAV.ULK1.DN in quantitative proteomic profiling (see 3.8.1), functional annotations to 
GO terms and an enrichment analysis were performed using DAVID. A significant enrichment of 14 biological processes could be detected (Table 12). Surprisingly, the analysis revealed a prominent regulation of spliceosome-associated processes including "mRNA splicing, via spliceosome," "spliceosomal snRNP assembly," “mRNA 5'-splice site recognition," and "histone exchange." The additional evaluation of the cellular components annotated to the differentially regulated proteins showed a significant enrichment of 37 GO terms (Table 13). Corresponding to the annotated biological processes, multiple spliceosome-associated components showed significant enrichment.

Table 12: Biological processes annotated to proteins with significant regulation in quantitative proteomic profiling after transduction with AAV.ULK1.DN

\begin{tabular}{|c|c|c|c|}
\hline GO term (biological process) & $\%$ & P-value & $\begin{array}{c}\text { fold } \\
\text { enrichment }\end{array}$ \\
\hline GO:0000387 spliceosomal snRNP assembly & 3.39 & 7.59E-04 & 21.97 \\
\hline GO:0043486 histone exchange & 2.54 & 0.001438709 & 51.27 \\
\hline GO:0098609 cell-cell adhesion & 5.08 & 0.011842779 & 4.37 \\
\hline GO:0044210 'de novo' CTP biosynthetic process & 1.69 & 0.012847345 & 153.82 \\
\hline GO:0042493 response to drug & 7.63 & 0.020893135 & 2.62 \\
\hline GO:0000398 mRNA splicing, via spliceosome & 3.39 & 0.025472706 & 6.28 \\
\hline GO:0090168 Golgi reassembly & 1.69 & 0.025531079 & 76.91 \\
\hline $\begin{array}{l}\text { GO:0009052 pentose-phosphate shunt, non-oxidative } \\
\text { branch }\end{array}$ & 1.69 & 0.031812237 & 61.53 \\
\hline GO:0000395 mRNA 5'-splice site recognition & 1.69 & 0.038053265 & 51.27 \\
\hline GO:0007049 cell cycle & 3.39 & 0.042533911 & 5.13 \\
\hline GO:0051683 establishment of Golgi localization & 1.69 & 0.044254416 & 43.95 \\
\hline GO:0007420 brain development & 5.08 & 0.046383606 & 3.05 \\
\hline GO:0016192 vesicle-mediated transport & 3.39 & 0.047969873 & 4.88 \\
\hline GO:0043066 negative regulation of apoptotic process & 6.78 & 0.04981385 & 2.38 \\
\hline GO:0045454 cell redox homeostasis & 2.54 & 0.064098477 & 7.21 \\
\hline GO:0006979 response to oxidative stress & 3.39 & 0.068389859 & 4.21 \\
\hline GO:0006098 pentose-phosphate shunt & 1.69 & 0.080637949 & 23.66 \\
\hline $\begin{array}{l}\text { GO:0046628 positive regulation of insulin receptor } \\
\text { signaling pathway }\end{array}$ & 1.69 & 0.080637949 & 23.66 \\
\hline GO:0033120 positive regulation of RNA splicing & 1.69 & 0.086566947 & 21.97 \\
\hline GO:0042542 response to hydrogen peroxide & 2.54 & 0.090175449 & 5.92 \\
\hline GO:0006754 ATP biosynthetic process & 1.69 & 0.092458046 & 20.51 \\
\hline GO:2000811 negative regulation of anoikis & 1.69 & 0.092458046 & 20.51 \\
\hline GO:0043149 stress fiber assembly & 1.69 & 0.098311484 & 19.23 \\
\hline GO:0009987 cellular process & 1.69 & 0.098311484 & 19.23 \\
\hline GO:0014070 response to organic cyclic compound & 4.24 & 0.099094499 & 2.83 \\
\hline
\end{tabular}

Lysates obtained from E18 rat cortical neurons on DIV 8 after transduction with AAV.mCherry or AAV.ULK1.DN were subjected to proteomic analysis. An enrichment analysis of functional annotations in Gene Ontology (GO) was performed for all significantly regulated proteins. The percentage of annotated proteins (\%), P-value, and fold enrichment value are given for each GO biological process term. Significantly regulated processes (Modified Fisher Exact P-value < 0.05) are outlined in orange. Table adapted from Vahsen et al. (2020), licensed under a CC BY 4.0 license, https://creativecommons.org/licenses/by/4.0/. 
Table 13: Cellular components annotated to proteins with significant regulation in quantitative proteomic profiling after transduction with AAV.ULK1.DN

\begin{tabular}{|c|c|c|c|}
\hline GO term (cellular component) & $\%$ & P-value & $\begin{array}{c}\text { fold } \\
\text { enrichment }\end{array}$ \\
\hline GO:0070062 extracellular exosome & 43.22 & $2.87 \mathrm{E}-14$ & 3.08 \\
\hline GO:0005737 cytoplasm & 47.46 & $6.11 \mathrm{E}-06$ & 1.71 \\
\hline GO:0005685 U1 snRNP & 4.24 & $7.78 \mathrm{E}-06$ & 38.01 \\
\hline GO:0005634 nucleus & 44.07 & $1.76 \mathrm{E}-05$ & 1.71 \\
\hline GO:0005683 U7 snRNP & 3.39 & $1.91 \mathrm{E}-05$ & 70.96 \\
\hline GO:0005913 cell-cell adherens junction & 8.47 & $3.45 \mathrm{E}-05$ & 6.16 \\
\hline GO:0005687 U4 snRNP & 3.39 & $6.37 \mathrm{E}-05$ & 49.12 \\
\hline GO:0034709 methylosome & 3.39 & $8.08 \mathrm{E}-05$ & 45.62 \\
\hline GO:0005739 mitochondrion & 20.34 & $1.15 \mathrm{E}-04$ & 2.39 \\
\hline GO:0034719 SMN-Sm protein complex & 3.39 & $1.78 \mathrm{E}-04$ & 35.48 \\
\hline GO:0005682 U5 snRNP & 3.39 & $2.10 \mathrm{E}-04$ & 33.61 \\
\hline GO:0005686 U2 snRNP & 3.39 & $3.77 \mathrm{E}-04$ & 27.77 \\
\hline GO:0005689 U12-type spliceosomal complex & 3.39 & $6.82 \mathrm{E}-04$ & 22.81 \\
\hline GO:0043209 myelin sheath & 5.93 & 0.00123469 & 5.85 \\
\hline GO:0005829 cytosol & 17.80 & 0.001785607 & 2.11 \\
\hline GO:0005654 nucleoplasm & 18.64 & 0.002315532 & 2.01 \\
\hline GO:0071013 catalytic step 2 spliceosome & 4.24 & 0.002909345 & 8.40 \\
\hline GO:0005925 focal adhesion & 7.63 & 0.003299353 & 3.63 \\
\hline GO:0016020 membrane & 21.19 & 0.004046228 & 1.81 \\
\hline GO:0071004 U2-type prespliceosome & 2.54 & 0.004891202 & 28.17 \\
\hline GO:0005794 Golgi apparatus & 11.02 & 0.005343389 & 2.51 \\
\hline GO:0030529 intracellular ribonucleoprotein complex & 4.24 & 0.006670896 & 6.65 \\
\hline GO:0048471 perinuclear region of cytoplasm & 9.32 & 0.006671261 & 2.74 \\
\hline GO:0005697 telomerase holoenzyme complex & 2.54 & 0.008141574 & 21.77 \\
\hline GO:0016607 nuclear speck & 4.24 & 0.012172845 & 5.58 \\
\hline GO:0005681 spliceosomal complex & 3.39 & 0.012660827 & 8.19 \\
\hline GO:0005743 mitochondrial inner membrane & 5.93 & 0.01345952 & 3.57 \\
\hline GO:0043234 protein complex & 8.47 & 0.01390254 & 2.61 \\
\hline GO:0071011 precatalytic spliceosome & 2.54 & 0.016791188 & 14.97 \\
\hline GO:0042995 cell projection & 3.39 & 0.021951496 & 6.65 \\
\hline GO:0044297 cell body & 3.39 & 0.022551973 & 6.58 \\
\hline GO:0030864 cortical actin cytoskeleton & 2.54 & 0.030521683 & 10.89 \\
\hline GO:0043005 neuron projection & 5.93 & 0.035947002 & 2.84 \\
\hline GO:0097526 spliceosomal tri-snRNP complex & 1.69 & 0.042671918 & 45.62 \\
\hline GO:0034715 pICln-Sm protein complex & 1.69 & 0.042671918 & 45.62 \\
\hline GO:0000243 commitment complex & 1.69 & 0.042671918 & 45.62 \\
\hline GO:0000790 nuclear chromatin & 4.24 & 0.045119862 & 3.71 \\
\hline GO:0016342 catenin complex & 1.69 & 0.054528856 & 35.48 \\
\hline GO:0005789 endoplasmic reticulum membrane & 6.78 & 0.058216972 & 2.30 \\
\hline GO:0005802 trans-Golgi network & 3.39 & 0.072181287 & 4.12 \\
\hline GO:0022625 cytosolic large ribosomal subunit & 3.39 & 0.091801362 & 3.71 \\
\hline
\end{tabular}

Lysates obtained from E18 rat cortical neurons on DIV 8 after transduction with AAV.mCherry or AAV.ULK1.DN were subjected to proteomic analysis. An enrichment analysis of functional annotations in Gene Ontology (GO) was performed for all significantly regulated proteins. The percentage of annotated proteins (\%), P-value, and fold enrichment value are given for each GO cellular components term. Significantly regulated processes (Modified Fisher Exact P-value < 0.05) are outlined in orange. Table adapted from Vahsen et al. (2020), licensed under a CC BY 4.0 license, https://creativecommons.org/licenses/by/4.0/. 
To more closely investigate the interactions between the significantly regulated proteins, a STRING database search was performed, which revealed a significant number of proteinprotein interactions (Figure 25A). More detailed analysis uncovered four protein clusters according to functions in "translation, ribosomal function," "spliceosome, RNA recognition," "protein kinase activity, intracellular signal transduction," and "ATP biosynthesis, mitochondrial function." Most of the proteins connected with ATP biosynthesis/mitochondrial function and translation displayed higher levels after transduction with AAV.ULK1.DN (Figure 25B). In contrast, half of the proteins associated with protein kinase activity and the spliceosome showed upregulation, while the other half was downregulated by AAV.ULK1.DN.

In summary, quantitative proteomic profiling therefore revealed a prominent regulation of proteins connected to splicing and translation by AAV.ULK1.DN.

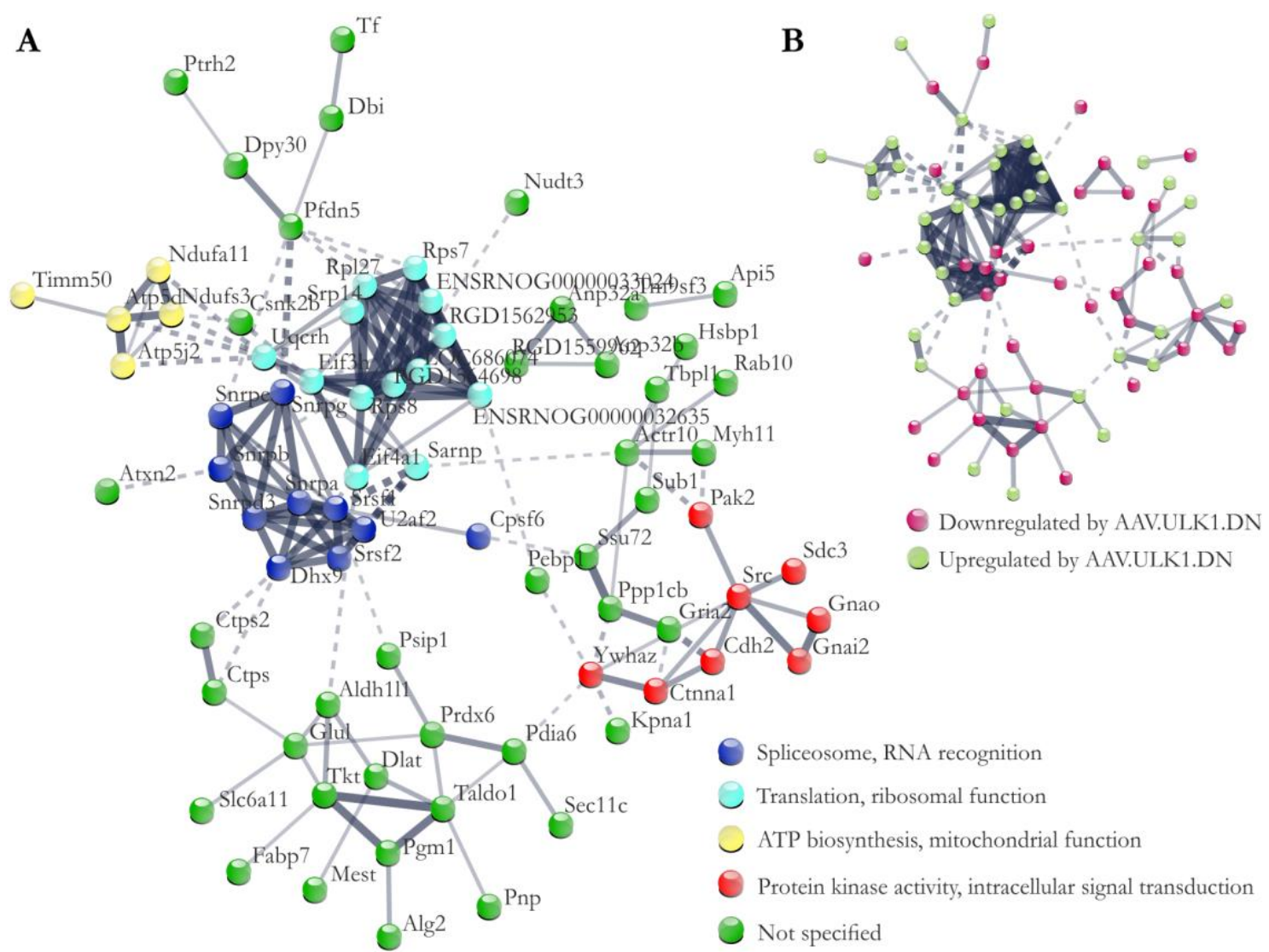

Figure 25: Overview of interactions between proteins with significant regulation in proteomic profiling after transduction with AAV.ULK1.DN. Lysates obtained from E18 rat cortical neurons on DIV 8 after transduction with AAV.mCherry or AAV.ULK1.DN were subjected to proteomic analysis. (A) Protein-protein network map of all significantly regulated proteins after transduction with AAV.ULK1.DN showing a significant number of interactions (STRING database enrichment P-value $=6.93 \mathrm{E}-07$ ) in comparison with AAV.mCherry. 4 clusters according to k-Means clustering are highlighted by different colors. (B) The same protein-protein network map as in A highlighting down- and upregulated proteins. Figure adapted from Vahsen et al. (2020), licensed under a CC BY 4.0 license, https://creativecommons.org/licenses/by/4.0/. 


\subsection{Investigation of splicing - transcriptomic analysis of cortical neurons transduced with AAV.ULK1.DN}

\subsubsection{AAV.ULK1.DN results in differential splicing}

The proteomic data surprisingly unraveled a distinct regulation of splicing-associated proteins by AAV.ULK1.DN. This is of particular interest, as dysregulated splicing is a wellknown pathophysiological feature of numerous neurodegenerative diseases. Therefore, one could speculate that AAV.ULK1.DN might elicit some of its neuroprotective and proregenerative effects via differential splicing of relevant degeneration or regenerationassociated genes. To investigate this hypothesis more closely, rat cortical neurons were transduced with AAV.mCherry and AAV.ULK1.DN and cultured for 8 DIV, after which a transcriptomic analysis was performed to assess differential exon expression.

Indeed, 36 out of 16551 sequenced genes showed differential exon usage after transduction with AAV.ULK1.DN compared with AAV.mCherry (Figure 26A). Unsurprisingly, the highest number of regulated exons per gene was found for Ulk1 (Figure 26B), with 24 out of 28 exons down- and 4 exons upregulated (Figure 27). Ulk1 was also upregulated on whole gene level (data not shown), confirming the overexpression of the CTD of ULK1 by AAV.ULK1.DN.

A

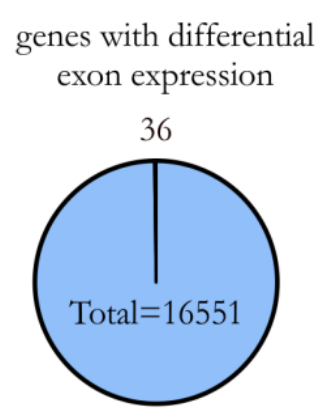

B

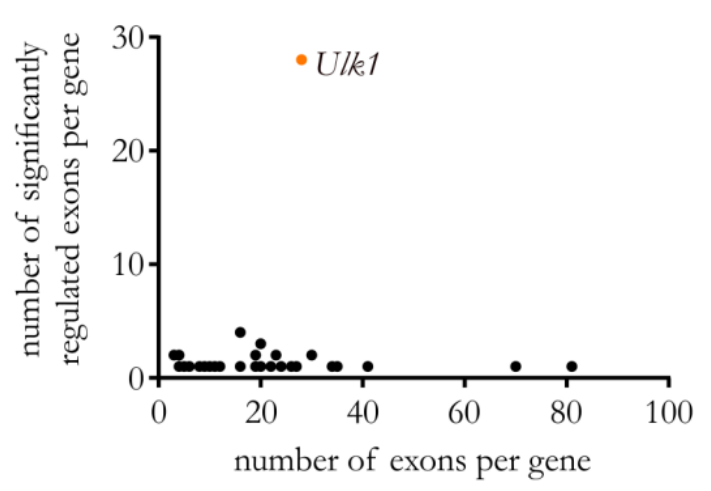

Figure 26: Differential exon expression analysis reveals AAV.ULK1.DN modulates differential splicing. Lysates obtained from E18 rat cortical neurons on DIV 8 after transduction with AAV.mCherry or AAV.ULK1.DN were subjected to differential exon expression analysis. (A) Pie chart showing the number of genes out of all sequenced genes with significantly (FDR-corrected P-value $<0.05$ ) differential exon expression after transduction with AAV.ULK1.DN ( $\mathrm{n}=3$ independent cultures). (B) Graph showing the number of significantly regulated exons per significantly regulated gene after transduction with AAV.ULK1.DN compared with the total number of exons per gene. All 28 exons of Ulk1 (orange) show significantly differential exon usage by AAV.ULK1.DN. Figure adapted from Vahsen et al. (2020), licensed under a CC BY 4.0 license, https://creativecommons.org/licenses/by/4.0/. 


\subsubsection{AAV.ULK1.DN modulates the splicing of axonal degeneration and regeneration-associated genes}

To investigate the biological relevance of the genes differentially spliced by AAV.ULK1.DN (see 3.9.1) more closely, an enrichment analysis of functional annotation to GO terms was performed using DAVID. A significant enrichment of twelve biological processes (Table 14), five molecular functions (Table 15), and seven cellular components (Table 16) could be detected. Strikingly, the analysis revealed a significant regulation of the neurite outgrowth and regeneration-associated processes "neuron projection regeneration," "axonogenesis," and "axon extension" (Table 14), which was also reflected by the enriched cellular components "growth cone" and "neuronal projection" (Table 16). Closer evaluation demonstrated reduced exon usage for specific genes with evident implications in axon regeneration, such as the MAPK pathway-associated gene MAPK activated protein kinase 3 (Mapkap3) and the CSPG signaling-related gene protein tyrosine phosphatase receptor type $\mathrm{f}$ (Ptprf) (Figure 27).

Table 14: Biological processes annotated to genes with significantly different exon usage after transduction with AAV.ULK1.DN

\begin{tabular}{|l|l|l|l|}
\hline \multicolumn{1}{|c|}{ GO term (biological process) } & \multicolumn{1}{c|}{$\%$} & \multicolumn{1}{c|}{ P-value } & \multicolumn{1}{c|}{$\begin{array}{c}\text { fold } \\
\text { enrichment }\end{array}$} \\
\hline $\begin{array}{l}\text { GO:0043525 positive regulation of neuron apoptotic } \\
\text { process }\end{array}$ & 8.33 & 0.007266673 & 22.63 \\
\hline $\begin{array}{l}\text { GO:0047497 mitochondrion transport along } \\
\text { microtubule }\end{array}$ & 5.56 & 0.010222829 & 188.55 \\
\hline GO:0085029 extracellular matrix assembly & 5.56 & 0.011916783 & 161.61 \\
\hline $\begin{array}{l}\text { GO:0050731 positive regulation of peptidyl-tyrosine } \\
\text { phosphorylation }\end{array}$ & 8.33 & 0.013109104 & 16.64 \\
\hline GO:0031102 neuron projection regeneration & 5.56 & 0.015296287 & 125.70 \\
\hline GO:0007409 axonogenesis & 8.33 & 0.016999073 & 14.50 \\
\hline GO:0001764 neuron migration & 8.33 & 0.018962936 & 13.68 \\
\hline GO:0008285 negative regulation of cell proliferation & 11.11 & 0.025528533 & 6.05 \\
\hline GO:0030182 neuron differentiation & 8.33 & 0.029412689 & 10.81 \\
\hline GO:0043547 positive regulation of GTPase activity & 11.11 & 0.029950269 & 5.68 \\
\hline GO:0046777 protein autophosphorylation & 8.33 & 0.040086141 & 9.12 \\
\hline GO:0048675 axon extension & 5.56 & 0.043574474 & 43.51 \\
\hline GO:0007274 neuromuscular synaptic transmission & 5.56 & 0.05011318 & 22.63 \\
\hline GO:1901216 positive regulation of neuron death & 5.56 & 0.05011318 & 188.55 \\
\hline GO:0007528 neuromuscular junction development & 5.56 & 0.059840284 & 161.61 \\
\hline GO:0048709 oligodendrocyte differentiation & 5.56 & 0.069471067 & 16.64 \\
\hline GO:0010506 regulation of autophagy & 5.56 & 0.074250626 & 125.70 \\
\hline GO:0042594 response to starvation & 5.56 & 0.086880387 & 14.50 \\
\hline GO:0007265 Ras protein signal transduction & 5.56 & 0.094689232 & 13.68 \\
\hline
\end{tabular}

E18 rat cortical neurons on DIV 8 after transduction with AAV.mCherry or AAV.ULK1.DN were analyzed for differential exon expression. An enrichment analysis of functional annotations to GO terms was performed for all genes with significantly differential exon usage. The percentage of annotated genes (\%), P-value, and fold enrichment value are given for each GO biological process term. Significantly regulated processes 
(Modified Fisher Exact P-value < 0.05) are outlined in orange. Table adapted from Vahsen et al. (2020), licensed under a CC BY 4.0 license, https://creativecommons.org/licenses/by/4.0/.

Table 15: Molecular functions annotated to genes with significantly different exon usage after transduction with AAV.ULK1.DN

\begin{tabular}{|l|l|l|l|}
\hline \multicolumn{1}{|c|}{ GO term (molecular function) } & \multicolumn{1}{c|}{$\%$} & \multicolumn{1}{c|}{ P-value } & $\begin{array}{c}\text { fold } \\
\text { enrichment }\end{array}$ \\
\hline GO:0008017 microtubule binding & 13.89 & $3.44 \mathrm{E}-04$ & 14.16 \\
\hline GO:0005515 protein binding & 25.00 & 0.002866453 & 3.37 \\
\hline GO:0005509 calcium ion binding & 13.89 & 0.024724913 & 4.27 \\
\hline GO:0016887 ATPase activity & 8.33 & 0.03348841 & 10.02 \\
\hline GO:0031593 polyubiquitin binding & 5.56 & 0.042905934 & 44.05 \\
\hline GO:0051879 Hsp90 protein binding & 5.56 & 0.053736955 & 34.98 \\
\hline GO:0048365 Rac GTPase binding & 5.56 & 0.059873015 & 31.30 \\
\hline GO:0005524 ATP binding & 16.67 & 0.071890511 & 2.54 \\
\hline GO:0030971 receptor tyrosine kinase binding & 5.56 & 0.079551717 & 23.32 \\
\hline
\end{tabular}

E18 rat cortical neurons on DIV 8 after transduction with AAV.mCherry or AAV.ULK1.DN were analyzed for differential exon expression. An enrichment analysis of functional annotations to GO terms was performed for all genes with significantly differential exon usage. The percentage of annotated genes (\%), P-value, and fold enrichment value are given for each GO molecular function term. Significantly regulated processes (Modified Fisher Exact P-value < 0.05) are outlined in orange. Table adapted from Vahsen et al. (2020), licensed under a CC BY 4.0 license, https://creativecommons.org/licenses/by/4.0/.

Table 16: Cellular components annotated to genes with significantly different exon usage after transduction with AAV.ULK1.DN

\begin{tabular}{|l|l|l|l|}
\hline \multicolumn{1}{|c|}{ GO term (cellular component) } & \multicolumn{1}{c|}{$\%$} & \multicolumn{1}{c|}{ P-value } & \multicolumn{1}{c|}{$\begin{array}{c}\text { fold } \\
\text { enrichment }\end{array}$} \\
\hline GO:0005737 cytoplasm & 52.78 & 0.002045852 & 1.92 \\
\hline GO:0030426 growth cone & 11.11 & 0.002495712 & 14.30 \\
\hline GO:0005874 microtubule & 11.11 & 0.009137822 & 8.97 \\
\hline GO:0030122 AP-2 adaptor complex & 5.56 & 0.01278247 & 151.18 \\
\hline GO:0043025 neuronal cell body & 13.89 & 0.016587416 & 4.90 \\
\hline GO:0043005 neuron projection & 11.11 & 0.034920727 & 5.39 \\
\hline GO:0005875 microtubule associated complex & 5.56 & 0.04492779 & 42.33 \\
\hline GO:0044295 axonal growth cone & 5.56 & 0.055415359 & 34.14 \\
\hline GO:0060076 excitatory synapse & 5.56 & 0.064069539 & 29.40 \\
\hline GO:0014069 postsynaptic density & 8.33 & 0.070532359 & 6.67 \\
\hline GO:0005768 endosome & 8.33 & 0.076799024 & 6.35 \\
\hline GO:0005905 clathrin-coated pit & 5.56 & 0.079453526 & 23.52 \\
\hline GO:0030136 clathrin-coated vesicle & 5.56 & 0.089572865 & 20.75 \\
\hline GO:0005871 kinesin complex & 5.56 & 0.091248891 & 20.35 \\
\hline
\end{tabular}

E18 rat cortical neurons on DIV 8 after transduction with AAV.mCherry or AAV.ULK1.DN were analyzed for differential exon expression. An enrichment analysis of functional annotations to GO terms was performed for all genes with significantly differential exon usage. The percentage of annotated genes (\%), P-value, and fold enrichment value are given for each GO cellular component term. Significantly regulated processes (Modified Fisher Exact P-value < 0.05) are outlined in orange. Table adapted from Vahsen et al. (2020), licensed under a CC BY 4.0 license, https://creativecommons.org/licenses/by/4.0/.

Additionally, various genes associated with axonal degeneration were found to be differentially spliced by AAV.ULK1.DN. This included genes with involvement in calcium homeostasis, the crucial initiating mechanism of AAD (see 1.2.1), such as TLC domain 
containing 1 (Tlcd1), agrin (Agrn), and fibulin 7 (Fbln 7 ) (Figure 27), which was also reflected by the enrichment of the GO molecular function term "calcium ion binding" (Table 15). Furthermore, a consistent enrichment of terms associated with microtubules and axonal transport, another mechanism with a critical role in both AAD (see 1.2.1) and growth cone formation during axon regeneration, was found, namely "mitochondrion transport along microtubule" (Table 14), "microtubule binding" (Table 15), "microtubule," and "microtubule associated complex" (both Table 16). This included genes such as kinesin family member 1b (Kif1b), kinesin family member 20a (Kif20a), Rho/Rac guanine nucleotide exchange factor 2 (Arhgef2), and microtubule-associated protein tau (Mapt) (Figure 27). Finally, the ER stress mediator DNA damage inducible transcript 3 (Ddit3) also showed decreased exon usage after transduction with AAV.ULK1.DN (Figure 27).

Taken together, the differential expression analysis revealed that AAV.ULK1.DN modulates the differential splicing of genes with key roles in axonal degeneration and regeneration, uncovering a previously unknown function of ULK1 in splicing.

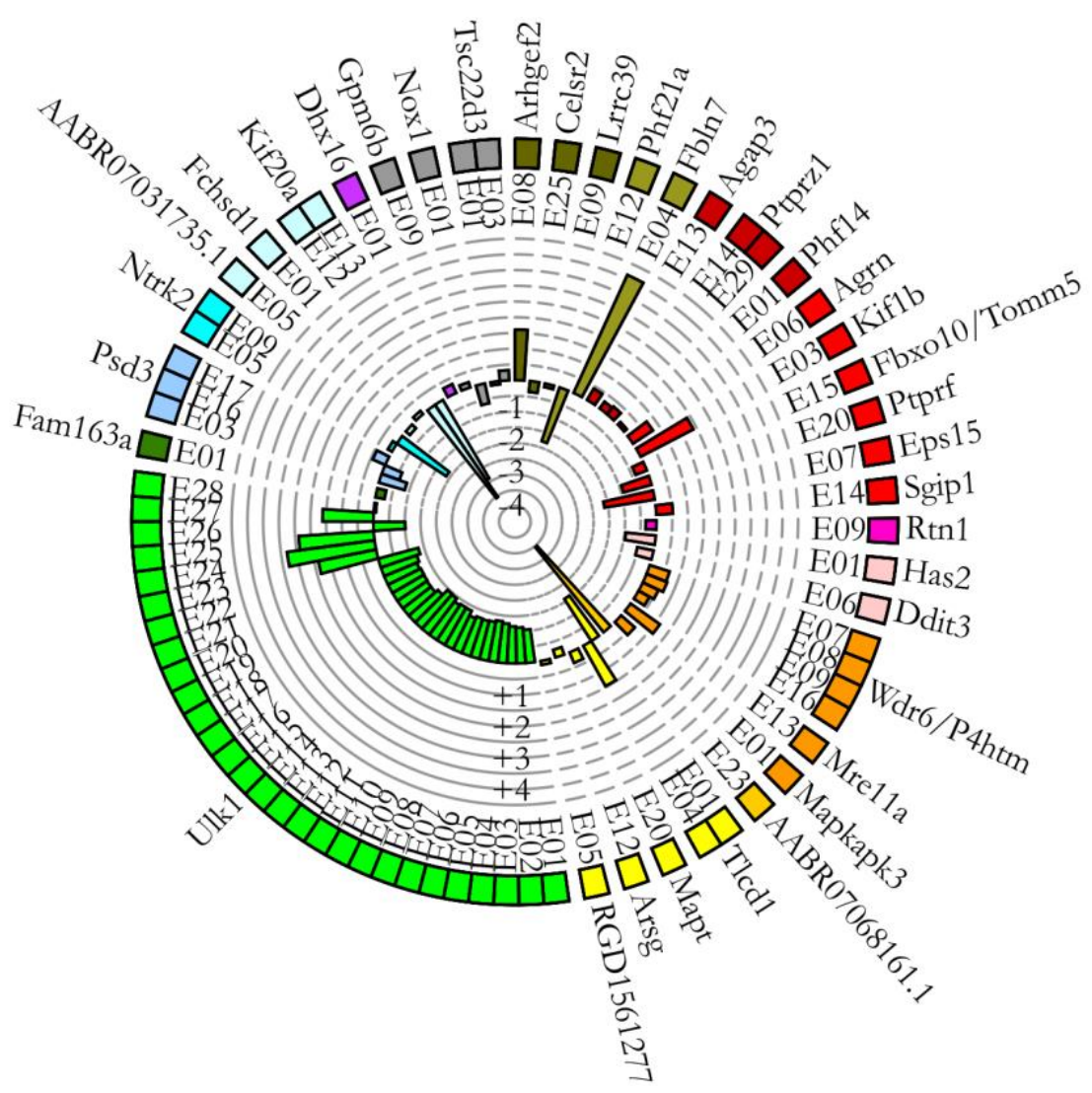

Figure 27: AAV.ULK1.DN mediates differential splicing of genes involved in axonal degeneration and regeneration. E18 rat cortical neurons on DIV 8 after transduction with AAV.mCherry or AAV.ULK1.DN were analyzed for differential exon expression. Circos graph showing the names of genes with differential exon usage after transduction with AAV.ULK1.DN. The differentially expressed exons are outlined for each gene (e.g. E01: Exon 1). Bars indicate the $\log _{2} \mathrm{fc}$ in expression for each exon by AAV.ULK1.DN. Circular grey lines 
indicate $\log _{2}$ fc from -4.5 to 4.5. Figure adapted from Vahsen et al. (2020), licensed under a CC BY 4.0 license, https://creativecommons.org/licenses/by/4.0/.

\subsection{AAV.ULK1.DN exerts neuroprotective and pro-regenerative effects by enhancing translation via an mTOR-S6-dependent mechanism}

In addition to a prominent regulation of splicing, the proteomic analysis identified a distinct

upregulation of proteins connected to translation (see 3.8). This finding raised interest in a more detailed characterization, considering that translation is widely implicated in axonal homeostasis and thus involved in axon degeneration and regeneration, as well as neurite outgrowth. Furthermore, a close interaction between ULK1 and mTOR, the master regulator of translation and autophagy, is well characterized (for details, see 1.3 and 1.4). Therefore, Western blot analyses of proteins belonging to the mTOR pathway were performed. To complement the results of the proteomic analysis, which technically cannot detect differences in phosphorylation, especially the levels of phospho-proteins were examined. Using the paradigm to study the effects of AAV.ULK1.DN in vitro established above (see 3.3.3.2), cortical neurons were transduced with AAV.ULK1.DN and AAV.mCherry, treated with rapamycin on DIV 7, and lysed on DIV 8 (Figure 28A).

Western blot analysis of the total mTOR levels showed no significant differences between both AAV vectors and after rapamycin treatment (Figure 28B). However, mTOR is finely regulated by phosphorylation, with phosphorylation at Ser2448 leading to mTOR activation. Indeed, the analysis of $\mathrm{p}-\mathrm{mTOR}$ revealed significantly higher expression after transduction with AAV.ULK1.DN (1.99 \pm 0.36 a.u.) when compared with AAV.mCherry, indicating higher levels of activated mTOR (Figure 28C). Administration of rapamycin significantly reduced $\mathrm{p}-\mathrm{m}$ TOR levels for cells transduced with both AAV vectors, even though a tendency to increased p-mTOR expression was also observed in rapamycin-treated conditions after transduction with AAV.ULK1.DN (0.44 \pm 0.05 a.u.), as compared with AAV.mCherry $(0.22 \pm 0.05$ a.u.).

To corroborate higher mTOR activation, the phosphorylation status of S6 (p-S6) was assessed, which is a downstream target of active mTOR and triggers the biosynthesis of ribosomal proteins and elongation factors required for translation. Indeed, transduction with AAV.ULK1.DN significantly increased p-S6 levels (1.46 \pm 0.12 a.u.) compared to AAV.mCherry (Figure 28D), confirming enhanced mTOR activity as the mechanism responsible for the upregulation of translational proteins by AAV.ULK1.DN. Treatment 
with rapamycin led to a very strong reduction in $\mathrm{p}-\mathrm{S} 6$ levels for both AAV vectors (AAV.mCherry: $0.06 \pm 0.02$ a.u., AAV.ULK1.DN: $0.05 \pm 0.01$ a.u.).

A

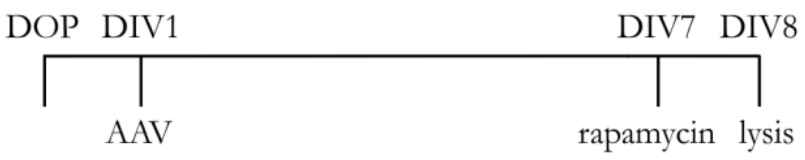

B
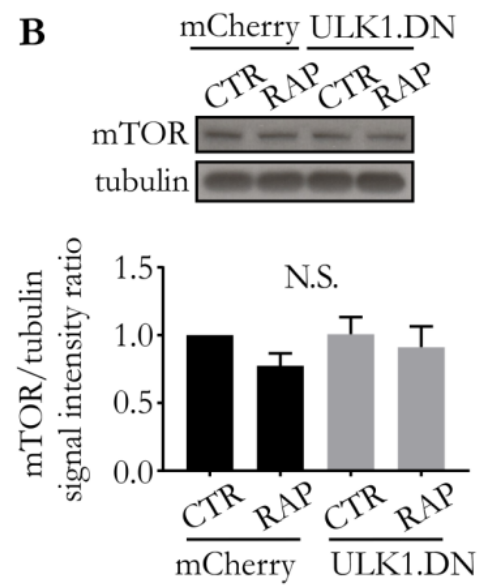

C
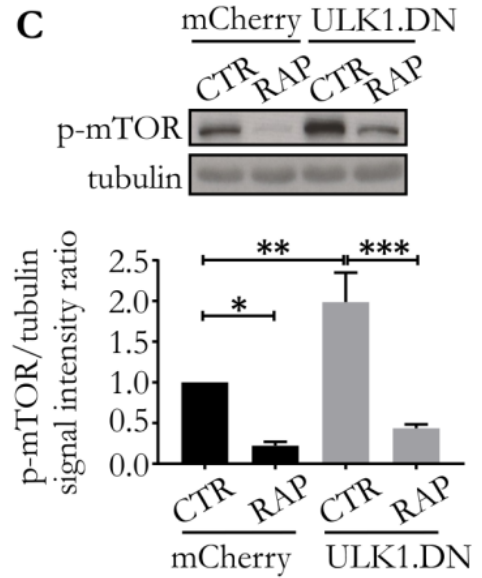

D
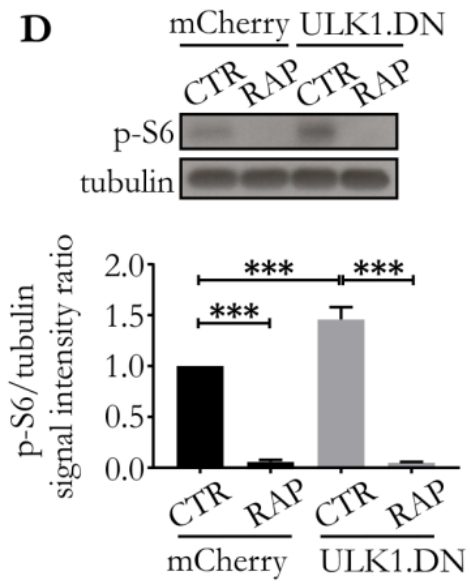

Figure 28: AAV.ULK1.DN enhances mTOR signaling. (A) Scheme of experimental setup. DOP: day of preparation of E18 rat cortical neurons. DIV: day in vitro. AAV: transduction with adeno-associated viral vectors. Rapamycin: addition of rapamycin (RAP, $750 \mathrm{nM}) 24$ hours before lysis. (B - D) Top: Representative Western blots of mTOR, p-mTOR and p-S6, and the corresponding bands of the loading control tubulin are shown. Bottom: Quantifications of the band intensities of mTOR, p-mTOR, and p-S6 (all $n=6$ independent cultures) normalized to tubulin as loading control. CTR: untreated control. Data are presented as means \pm SEM. $* \mathrm{P}<0.05,{ }^{*} \mathrm{P}<0.01, * * * \mathrm{P}<0.001$, N.S.: no significant difference, according to one-way ANOVA and Sidak's multiple comparisons test. Figure adapted from Vahsen et al. (2020), licensed under a CC BY 4.0 license, https://creativecommons.org/licenses/by/4.0/.

To understand the molecular mechanisms by which AAV.ULK1.DN enhances mTOR signaling in more detail, the levels of upstream regulators of mTOR (for details, see 1.2.3) were additionally assessed by Western blotting. Specifically, AMPK signaling and the PI3KAKT pathway, including the associated kinase GSK3 $\beta$, were evaluated.

Firstly, the expression of AMPK $\alpha$, the main subunit of AMPK that exerts an inhibitory effect on mTOR and additionally interacts with ULK1 directly (see 1.3), was analyzed. Western blot analysis of AMPK $\alpha$ showed no significant difference between AAV.ULK1.DN and AAV.mCherry in naïve and rapamycin-treated conditions (Figure 29A). However, the assessment of the phosphorylated (Thr172), thus active form of AMPK $\alpha$ (p-AMPK $\alpha)$ revealed significantly lower levels in naïve conditions after transduction with AAV.ULK1.DN (0.64 \pm 0.08 a.u.) as compared with AAV.mCherry (Figure 29B), suggesting that AAV.ULK1.DN enhances mTOR signaling and translation through a reduction in AMPK-mediated negative feedback. In line with this result, a trend to reduced p-AMPK 
expression was visible after rapamycin administration in AAV.ULK1.DN-transduced neurons (0.70 \pm 0.14 a.u.) when compared with AAV.mCherry (1.00 \pm 0.11 a.u.), which, however, did not reach statistical significance $(\mathrm{p}=0.107)$.

A
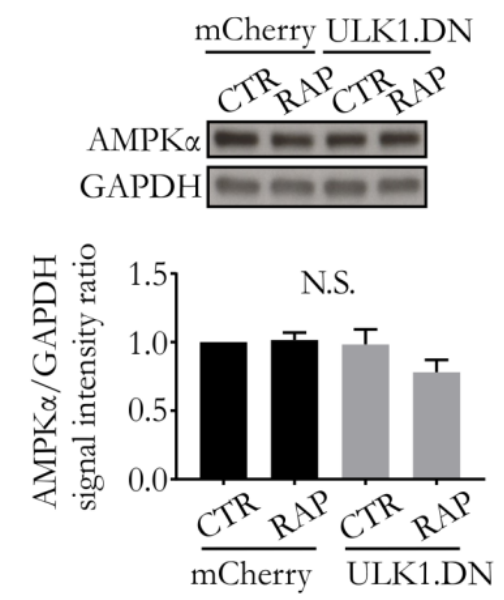

B
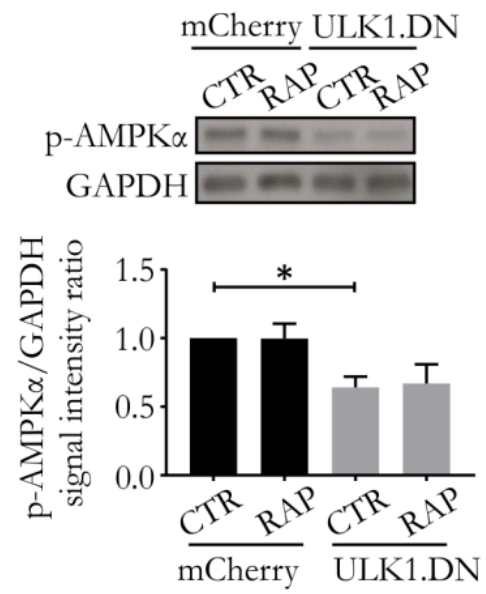

Figure 29: AAV.ULK1.DN reduces the levels of p-AMPK. (A, B) Top: Representative Western blots of AMPK $\alpha$ and $\mathrm{p}$-AMPK $\alpha$, and the corresponding bands of the loading control GAPDH are shown. Bottom: Quantifications of the band intensities of AMPK $\alpha$ ( $n=6$ independent cultures) and p-AMPK $\alpha(n=4-5$ independent cultures) normalized to GAPDH as loading control. CTR: untreated control. RAP: treated with rapamycin. Data are presented as means \pm SEM. $* \mathrm{P}<0.05$, N.S.: no significant difference, according to oneway ANOVA and Sidak's multiple comparisons test. Figure adapted from Vahsen et al. (2020), licensed under a CC BY 4.0 license, https://creativecommons.org/licenses/by/4.0/.

Lastly, the PI3K-AKT pathway, which mediates mTOR activation and is implicated in cell survival and growth, was analyzed. Western blot analysis of AKT showed no significant differences (Figure 30A). Surprisingly, the assessment of active AKT (p-AKT, Ser473) revealed significantly lower levels after transduction with AAV.ULK1.DN (0.82 \pm 0.06 a.u.) when compared with AAV.mCherry (Figure 30B), indicating a potential negative feedback loop between AAV.ULK1.DN-induced mTOR signaling and AKT activation. Treatment with rapamycin resulted in significantly lower levels after transduction with both AAV vectors. 
A

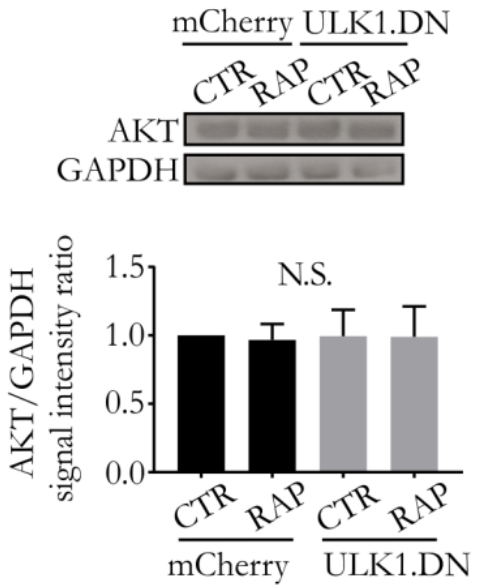

B
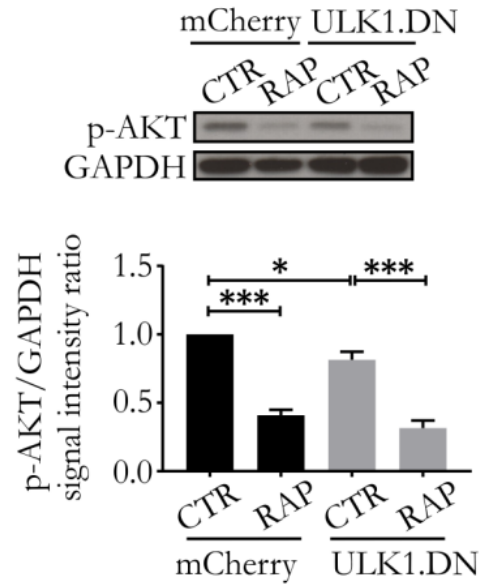

Figure 30: AAV.ULK1.DN moderately reduces p-AKT levels. (A, B) Top: Representative Western blots of AKT and p-AKT, and the loading control GAPDH. Bottom: Quantifications of the band intensities of AKT ( $\mathrm{n}=4$ independent cultures) and $\mathrm{p}-\mathrm{AKT}$ ( $\mathrm{n}=6$ independent cultures) normalized to GAPDH. CTR: untreated control. RAP: treated with rapamycin. Data are presented as means \pm SEM. $* \mathrm{P}<0.05,{ }^{*} * \mathrm{P}<0.001$, N.S.: no significant difference, according to one-way ANOVA and Sidak's multiple comparisons test. Figure adapted from Ribas et al. (2021), licensed under a CC BY 4.0 license, https://creativecommons.org/licenses/by/4.0/.

To study whether the reduction in $\mathrm{p}$-AKT levels after transduction with AAV.ULK1.DN was mediated by an effect on an upstream regulator of AKT, the levels of PTEN were analyzed, which is known to negatively regulate the AKT cascade and has additionally been implicated in axon regeneration. However, neither PTEN nor its phosphorylated form (p-PTEN) showed a significant regulation in Western blot analysis (Figure 31A, B), indicating that downregulation of $\mathrm{p}-\mathrm{AKT}$ by AAV.ULK1.DN is independent of PTEN.

A
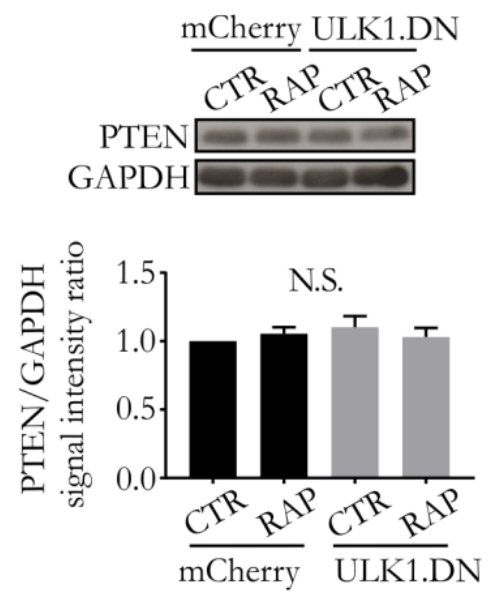

B
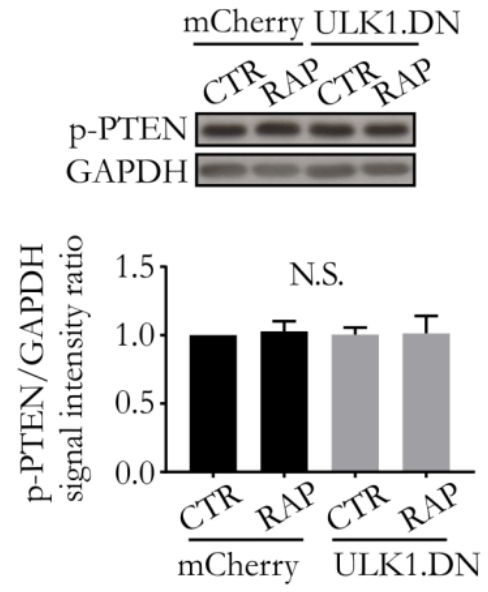

Figure 31: PTEN levels are not altered by AAV.ULK1.DN. (A, B) Top: Representative Western blots of PTEN and p-PTEN, and the loading control GAPDH. Bottom: Quantifications of the band intensities of PTEN ( $\mathrm{n}=4$ independent cultures) and p-PTEN ( $\mathrm{n}=6$ independent cultures) normalized to GAPDH. CTR: untreated control. RAP: treated with rapamycin. Data are presented as means \pm SEM. N.S.: no significant 
difference, according to one-way ANOVA and Sidak's multiple comparisons test. Figure adapted from Ribas et al. (2021), licensed under a CC BY 4.0 license, https://creativecommons.org/licenses/by/4.0/.

A
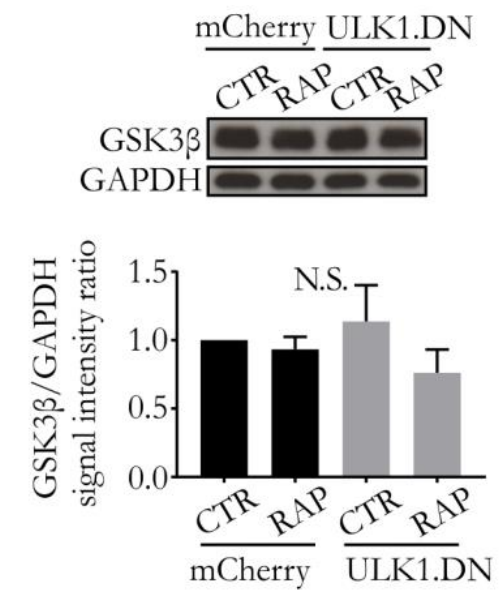

B
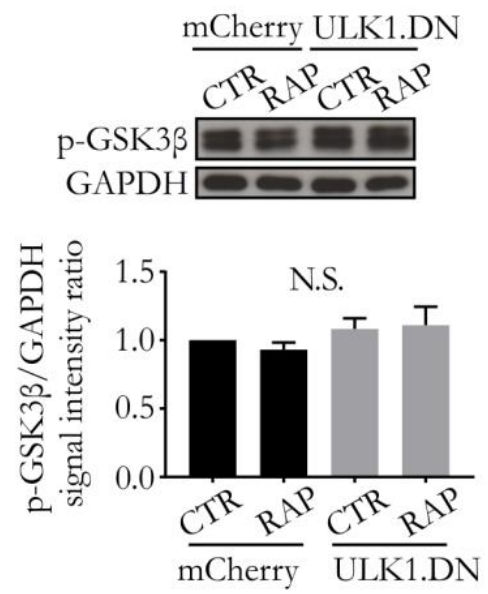

Figure 32: GSK3 $\beta$ expression is not altered by transduction with AAV.ULK1.DN. (A, B) Top: Representative Western blots of GSK3 $\beta$ and $\mathrm{p}-\mathrm{GSK} 3 \beta$, and the loading control GAPDH. Bottom: Quantifications of the band intensities of GSK3 $\beta$ ( $\mathrm{n}=6$ independent cultures) and p-GSK3 $\beta$ ( $\mathrm{n}=6$ independent cultures) normalized to GAPDH. CTR: untreated control. RAP: treated with rapamycin. Data are presented as means \pm SEM. N.S.: no significant difference, according to one-way ANOVA and Sidak's multiple comparisons test. Figure adapted from Ribas et al. (2021), licensed under a CC BY 4.0 license, https://creativecommons.org/licenses/by/4.0/. 
Having observed a regulation of several proteins involved in the PI3K-AKT-mTOR network, one could speculate that the levels of GSK3 $\beta$ might be altered as well, given the numerous interactions. However, Western blot analysis of GSK3 $\beta$ and its phosphorylated form (p-GSK3 $\beta$ ) showed no significant differences (Figure 32A, B), indicating that AAV.ULK1.DN regulates AKT and mTOR independent of GSK3 $\beta$.

Taken together, AAV.ULK1.DN therefore exerts neuroprotective and pro-regenerative effects also by enhancing translation via an mTOR-S6-dependent mechanism.

\subsection{AAV.ULK1.DN fosters axon growth and regeneration via increased ERK1 activity}

In addition to mTOR-dependent translation, the MAPK pathway is of great importance for cell growth and neuronal survival. Since differential splicing of Mapkap3 was observed after transduction with AAV.ULK1.DN (see 3.9.2), it seemed interesting to assess MAPK signaling in more detail. Specifically, ERK and JNK were analyzed, owing to their relevance in axon growth and regeneration.

A

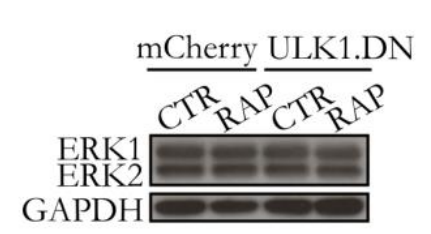

D

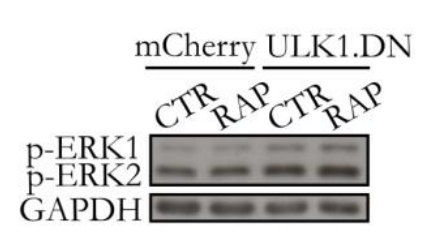

B

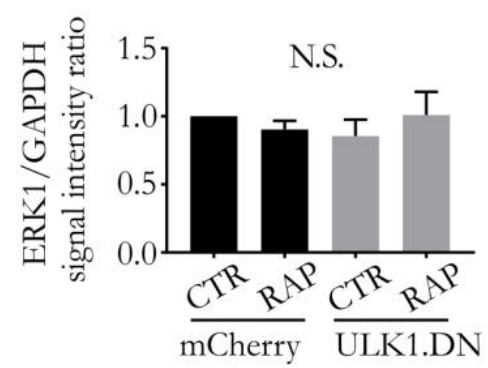

$\mathbf{E}$

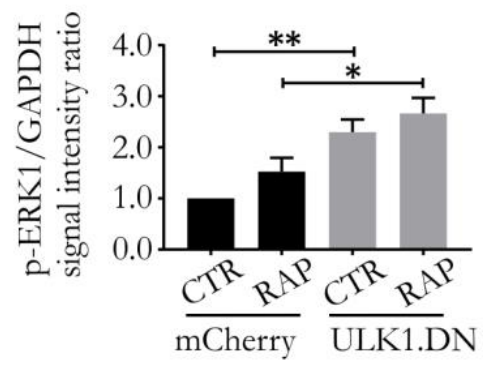

C

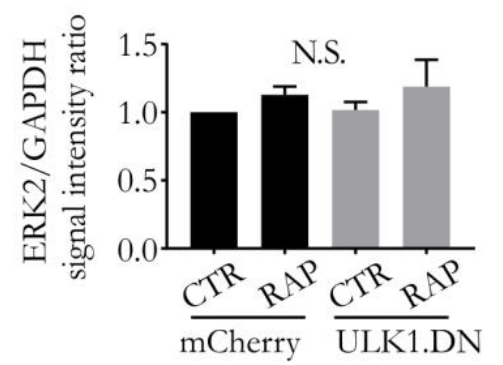

F

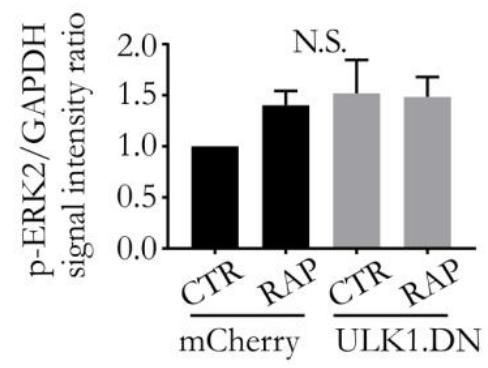

Figure 33: AAV.ULK1.DN increases the levels of p-ERK1. (A, D) Representative Western blots of ERK1/2, p-ERK1/2, and the loading control GAPDH. (B, C, E, F) Quantifications of the band intensities of ERK1/2 ( $n=6$ independent cultures) and p-ERK1/2 ( $n=6$ independent cultures) normalized to GAPDH. CTR: untreated control. RAP: treated with rapamycin. Data are presented as means \pm SEM. $* \mathrm{P}<0.05$, $* * \mathrm{P}<0.01$, N.S.: no significant difference, according to one-way ANOVA and Sidak's multiple comparisons test. Figure adapted from Ribas et al. (2021), licensed under a CC BY 4.0 license, https://creativecommons.org/licenses/by/4.0/. 
Assessment of the two isoforms ERK1/2 by Western blotting showed no significant differences (Figure 33A-C). However, the analysis of phosphorylated and thus active ERK1/2 (p-ERK1/2, Thr202/Tyr204) revealed significantly higher levels after transduction with AAV.ULK1.DN (untreated: $2.30 \pm 0.25$ a.u.; treated: $2.67 \pm 0.30$ a.u.), as compared with AAV.mCherry (treated: $1.52 \pm 0.27$ a.u.) in both naïve and rapamycin-treated conditions (Figure 33D, E), suggesting that increased ERK1 activation represents another mechanism by which AAV.ULK1.DN enhances axon growth-promoting effects. The analysis of p-ERK2 did not show any significant differences (Figure 33D, F).

Additionally, the levels of JNK, another MAPK with implications in neurite growth and regeneration, were analyzed after transduction with AAV.ULK1.DN. However, Western blot analysis of its two active phosphorylated isoforms (p-54/46-JNK) showed no significant regulation after transduction with AAV.ULK1.DN or rapamycin treatment (Figure 34A - C), indicating that AAV.ULK1.DN elicits its beneficial effects independent of JNK signaling.

In summary, these data suggest that AAV.ULK1.DN additionally exerts growth-promoting effects by enhanced activation of ERK1.

A

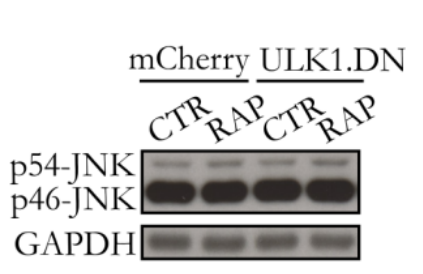

B

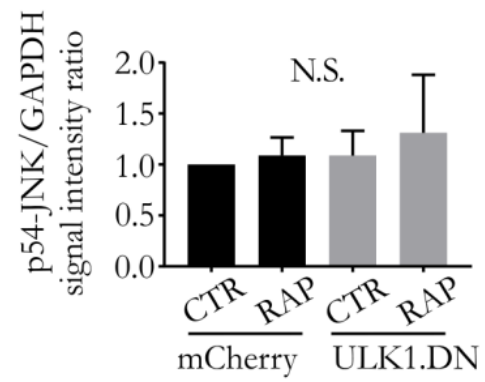

C

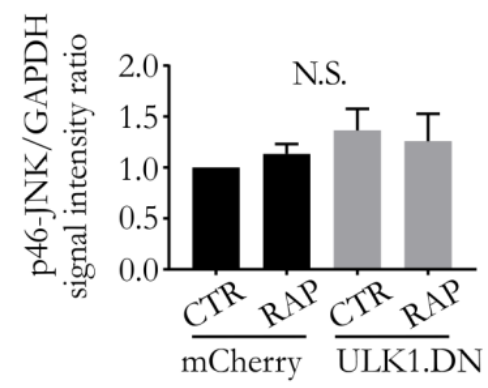

Figure 34: JNK levels are not altered by AAV.ULK1.DN. (A) Representative Western blot of p-JNK and the loading control GAPDH. (B, C) Quantifications of the band intensities of the two isoforms p54-JNK and p46-JNK (both $n=5-6$ independent cultures) normalized to GAPDH. CTR: untreated control. RAP: treated with rapamycin. Data are presented as means \pm SEM. N.S.: no significant difference, according to one-way ANOVA and Sidak's multiple comparisons test. Figure adapted from Ribas et al. (2021), licensed under a CC BY 4.0 license, https://creativecommons.org/licenses/by/4.0/.

\subsection{AAV.ULK1.DN counteracts inhibitory environmental signaling after lesion via downregulation of ROCK2}

The RhoA-ROCK2 pathway is a key regulator of the actin cytoskeleton and mediates the intracellular cascade underlying inhibitory environmental signaling after lesion, by which extrinsic molecules such as CSPG induce axonal degeneration and inhibit axonal 
regeneration and neurite outgrowth. The positive result of rescued neurite outgrowth on CSPG by AAV.ULK1.DN (see 3.7), together with differential splicing of the CSPG-related gene Ptprf (see 3.9.2), sparked interest in the molecular mechanisms underlying this effect. Western blot analysis of ROCK2 revealed significantly lower levels by AAV.ULK1.DN in both untreated $(0.57 \pm 0.08$ a.u.) and treated conditions ( $0.31 \pm 0.06$ a.u.), as compared with AAV.mCherry (treated: $0.82 \pm 0.10$ a.u.) (Figure 35), indicating that AAV.ULK.DN counteracts inhibitory environmental signaling through downregulation of ROCK2.

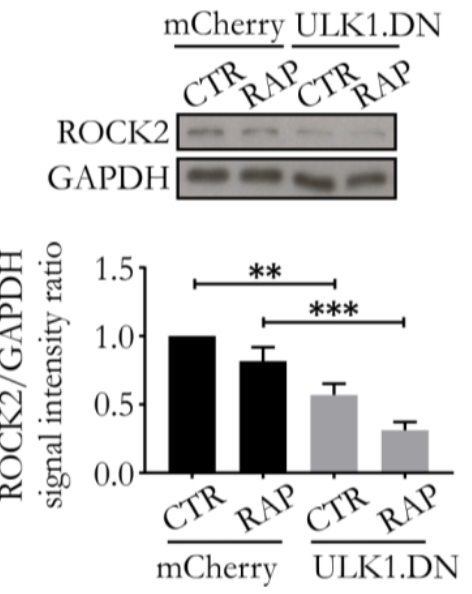

Figure 35: AAV.ULK1.DN downregulates ROCK2 levels. Top: Representative Western blot of ROCK2 and the loading control GAPDH. Bottom: Quantification of the band intensities of ROCK2 ( $\mathrm{n}=5$ independent cultures) normalized to GAPDH. CTR: untreated control. RAP: treated with rapamycin. Data are presented as means \pm SEM. ${ }^{* * P}<0.01,{ }^{* * * P}<0.001$, according to one-way ANOVA and Sidak's multiple comparisons test. Figure adapted from Ribas et al. (2021), licensed under a CC BY 4.0 license, https://creativecommons.org/licenses/by/4.0/.

A
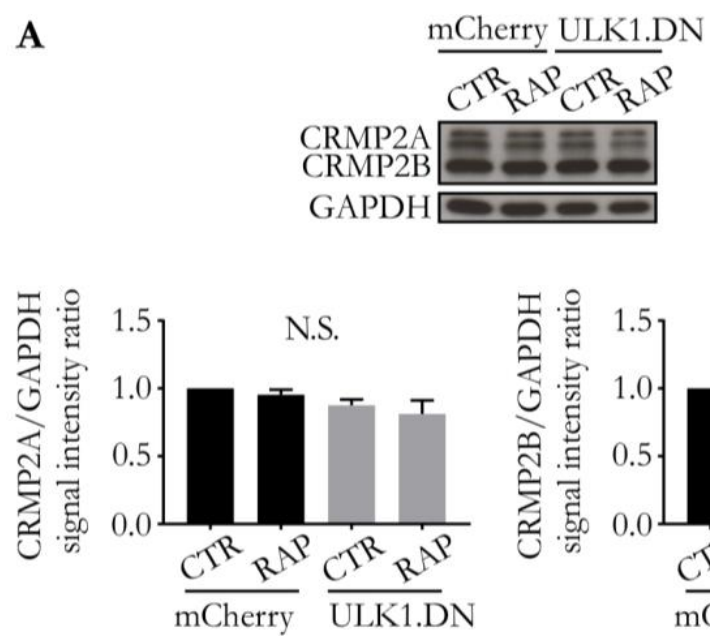

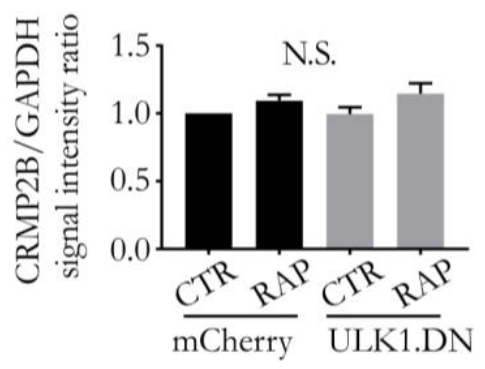

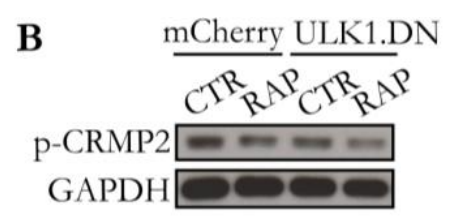

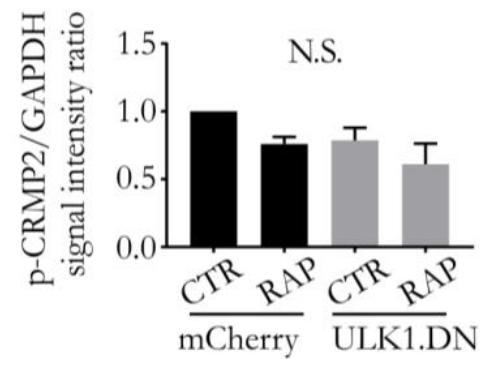

Figure 36: CRMP2 levels are not altered by AAV.ULK1.DN. (A, B) Top: Representative Western blots of CRMP2 and p-CRMP2, and the loading control GAPDH. Bottom: Quantifications of the band intensities of CRMP2A/B ( $\mathrm{n}=6$ independent cultures) and $\mathrm{p}-\mathrm{CRMP} 2$ ( $\mathrm{n}=5$ independent cultures) normalized to GAPDH. CTR: untreated control. RAP: treated with rapamycin. Data are presented as means \pm SEM. N.S.: no significant 
difference, according to one-way ANOVA and Sidak's multiple comparisons test. Figure adapted from Ribas et al. (2021), licensed under a CC BY 4.0 license, https://creativecommons.org/licenses/by/4.0/. 
ROCK2 activation leads to inhibition of CRMP2, resulting in microtubule disassembly and thus increased axonal degeneration and reduced axon outgrowth. To assess whether the observed downregulation of ROCK2 might alter CRMP2 expression, Western blot analyses of CRMP2 and its phosphorylated form (p-CRMP2) were performed (Figure 36A, B). However, no significant regulation of the two isoforms CRMP2A/B or p-CRMP2 was detectable, indicating that AAV.ULK1.DN exerts its beneficial effects independent of CRMP2.

\subsection{AAV.ULK1.DN does not enhance JAK-STAT3 signaling}

Another pathway of major importance for the control of cell growth and apoptosis is the JAK-STAT3 pathway. To assess whether AAV.ULK1.DN might also modulate JAK-STAT3 signaling, Western blots of STAT3 and its active phosphorylated form (p-STAT3) were performed. The assessment of the two isoforms STAT3 $\alpha$ and STAT3 $\beta$ revealed no significant regulation (Figure 37A). Interestingly, Western blot analysis of p-STAT3 showed significantly lower levels by AAV.ULK1.DN (untreated: $0.69 \pm 0.12$ a.u.), as compared with AAV.mCherry (Figure 37B), indicating a potential negative feedback mechanism between STAT3 signaling and the various other molecular targets regulated by AAV.ULK1.DN. Furthermore, the addition of rapamycin significantly reduced p-STAT3 levels in cells transduced with both AAV.ULK1.DN and AAV.mCherry. Taken together, these data suggest that AAV.ULK1.DN elicits its pro-regenerative effects independent of STAT3 signaling.

A
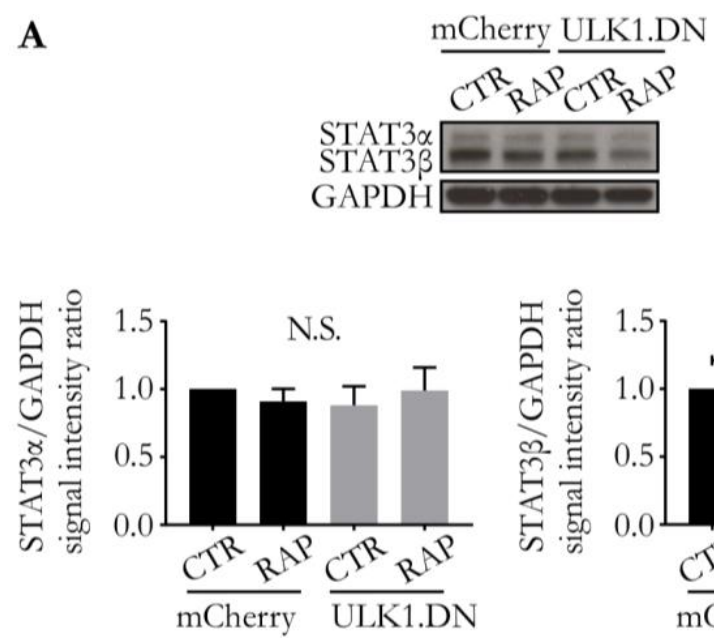

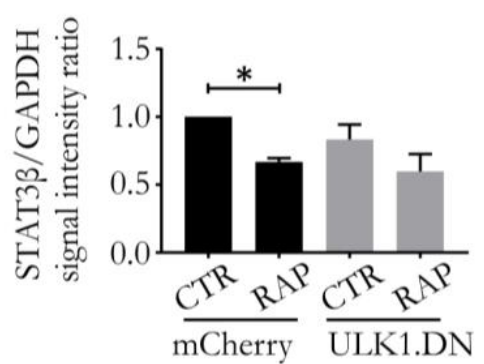

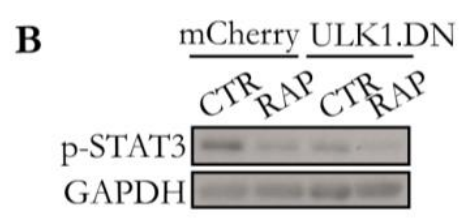

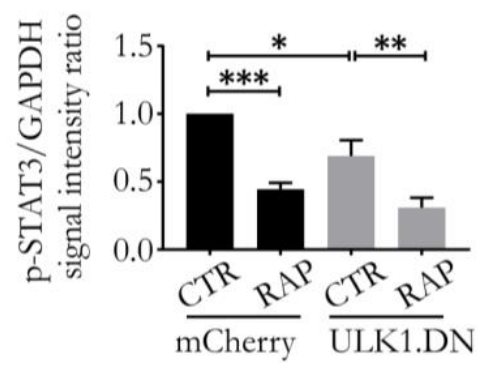

Figure 37: AAV.ULK1.DN moderately reduces p-STAT3 levels. (A, B) Top: Representative Western blots of STAT3 $\alpha / \beta$, p-STAT3, and the loading control GAPDH. Bottom: Quantifications of the band intensities of STAT $3 \alpha / \beta$ ( $\mathrm{n}=6$ independent cultures) and p-STAT3 ( $\mathrm{n}=5$ independent cultures) normalized to GAPDH. CTR: untreated control. RAP: treated with rapamycin. Data are presented as means $\pm \mathrm{SEM}$. $* \mathrm{P}<0.05$, $* * \mathrm{P}<0.01,{ }^{* * * \mathrm{P}}<0.001$, N.S.: no significant difference, according to one-way ANOVA and Sidak's multiple 
comparisons test. Figure adapted from Ribas et al. (2021), licensed under a CC BY 4.0 license, https://creativecommons.org/licenses/by/4.0/. 


\section{Discussion}

Neuron loss is a key feature of traumatic and degenerative CNS disorders leading to progressive clinical disability. Causal therapies, however, are currently not available. In the pathophysiological cascade of these disorders, axons degenerate comparably early, whereas the neuronal soma is affected at later stages of the disease course. The regeneration of degenerating axons is additionally severely restricted in the CNS, owing to a low intra-axonal regenerative capacity and extrinsic growth-inhibiting factors presented by glial cells after axonal injury. Therefore, a better understanding of the mechanisms of axonal degeneration and regeneration in the CNS is fundamental to develop new therapeutic avenues against neurodegeneration.

As a catabolic cellular pathway of crucial importance for neuronal and axonal homeostasis, autophagy is tightly connected to axonal degenerative and regenerative processes. However, both harmful and protective properties of autophagy have been shown in this context, rendering it unclear whether autophagy activation or inhibition should be pursued as a therapeutic strategy. Interestingly, a previous study (Ribas et al. 2015) demonstrated the strong accumulation of autophagic proteins, most prominently for the autophagy-initiating kinase ULK1, in degenerating axons after SCI. This finding raised the question of whether ULK1-dependent autophagy is a mediator of axonal degeneration after lesion or, conversely, if it represents an axon-protective mechanism. Based on a previous study in the laboratory showing axonal protection through unspecific autophagy inhibition (Knoferle et al. 2010), it was speculated that ULK1-dependent autophagy is a key executing process during axon degeneration. To test this hypothesis, the present study investigated the effects of AAV.ULK1.DN-mediated ULK1 inhibition on axonal degeneration and regeneration in vitro and delineated the underlying molecular mechanisms.

\subsection{Establishing an experimental paradigm to model autophagy induction after axonal injury in vitro}

Firstly, a reliable in vitro paradigm was sought to mimic the autophagy induction previously observed after SCI and to evaluate the effect of ULK1 inhibition in this model. In a first approach, a scratch lesion was performed on cultured rat cortical neurons to mechanically induce axonal degeneration. However, no reproducible induction of autophagic markers was observed, even though different culture durations and multiple time points were tested. These results are not in line with a previous publication that reported on the induction of autophagy after mechanical lesion in vitro, as demonstrated by increased LC3-II levels and 
reduced p62 expression (Han et al. 2014). It needs to be pointed out, however, that even though the scratch assay performed by Han et al. (2014) per se seems to have been similar to the method used here, the results were obtained $48 \mathrm{~h}$ after mechanical lesion to HT-22 cells. Therefore, it remains to be determined if the absence of an effect in this thesis needs to be attributed to a principally different biological response of cultured rat cortical neurons and the HT-22 cell line, or whether longer time points (e.g. $48 \mathrm{~h}$ ) after injury would be required to induce an effect in primary neurons. However, this thesis aimed to mimic the events observed after SCI, in which most autophagic proteins including ULK1 peaked within $24 \mathrm{~h}$ after injury, and thus required a more acute paradigm.

Hence, a second experimental approach was tested by applying the calcium ionophore A23187 onto cultured primary cortical neurons, aiming to replicate the increased calcium influx observed directly after axonal lesion that precedes autophagy activation in vivo. However, neither different incubation times nor different culture durations resulted in a reproducible induction of autophagy. In line with this result, there is no clear consensus in the literature regarding the effects of A23187 treatment on cultured cells. While an induction of autophagy was observed after A23187 administration to HCT116 cells for $24 \mathrm{~h}$ (Ding et al. 2007), treatment of neuroretina organotypic cultures with A23187 for $24 \mathrm{~h}$ led to the blockage of autophagy (Rodriguez-Muela et al. 2015). Effects of calcium ionophore treatment on autophagy therefore seem to widely depend on the experimental paradigm, rendering A23187 administration unsuitable to mimic autophagy activation in primary cortical neurons in vitro.

As both mechanical lesion and application of the calcium ionophore did not result in the reliable induction of autophagy, a third approach was tested by performing pharmacological activation of autophagy using the mTOR inhibitor rapamycin. While a moderate induction of autophagy was already observed after the application of $250 \mathrm{nM}$ rapamycin onto primary neurons for $6 \mathrm{~h}$, indicated by increased LC3-II levels, treatment with $750 \mathrm{nM}$ for $24 \mathrm{~h}$ additionally led to reduced p62 levels, demonstrating the successful induction of autophagy. Rapamycin is well known to induce autophagy (Klionsky et al. 2016) and the effect demonstrated here corresponds well to the peak of the majority of autophagic proteins previously observed $24 \mathrm{~h}$ after SCI (Ribas et al. 2015); therefore, this paradigm was used for the following investigation of the effects of ULK1 inhibition on autophagy. 


\subsection{The effect of AAV.ULK1.DN on neuronal autophagy}

To inhibit ULK1 in vitro, primary cortical neurons were transduced with an AAV vector expressing a dominant-negative form of ULK1 (AAV.ULK1.DN). Firstly, initial experiments were performed to demonstrate that both AAV.ULK1.DN and the corresponding control vector AAV.mCherry were successfully expressed, exerted only minor toxicity, and led to equal transduction rates. Hereafter, the effect of AAV.ULK1.DN-mediated ULK1 inhibition on autophagic proteins was evaluated in naïve and rapamycin-treated conditions using the paradigm of autophagy induction established in this thesis.

Expectedly, AAV.ULK1.DN led to a moderate reduction of autophagy, as demonstrated by significantly lower LC3-II expression and higher p62 levels in rapamycin-treated conditions. This finding confirms the results from previous experiments with siRNA-mediated knockdown of ULK1 (Chan et al. 2007; Chan et al. 2009) and ULK1 knockout (Wei et al. 2018), in which a moderate inhibition of autophagy, rather than a complete blockage, was reported. Interestingly, in this study, untreated control conditions also showed significantly increased levels of p62 after transduction with AAV.ULK1.DN, while no alterations in the expression of LC3-II, ATG5, or ATG7 were observed. LC3 levels also remained unchanged in naïve cells in previous experiments using ULK1.DN or siRNA against ULK1 (Chan et al. 2007; Chan et al. 2009), but p62, ATG5, or ATG7 expression were not analyzed in those studies. Interestingly, ULK1-dependent LC3-independent autophagy has previously been described in mammalian cells (Honda et al. 2014; Nishida et al. 2009). These data could therefore indicate that, in basal conditions, AAV.ULK1.DN only affects LC3-independent autophagy, while an effect on conventional LC3-mediated autophagy is unmasked under autophagy-induced conditions after rapamycin administration.

\subsection{Cell survival is not affected by AAV.ULK1.DN in vitro}

Considering the crucial homeostatic role of autophagy in neuron survival, the effects of AAV.ULK1.DN on cell survival were evaluated. No significant differences in the expression of the apoptosis marker cleaved caspase 3 were observed in neurons transduced with AAV.ULK1.DN in both unstimulated conditions and after rapamycin treatment, as compared with control. Similarly, transduction with AAV.ULK1.DN did not affect cytotoxicity after induction of apoptosis using staurosporine. In contrast to these results, injection of AAV.ULK1.DN into the midbrain of mice improved neuronal survival of dopaminergic nigral neurons in a model of PD (Balke et al. 2020). Similarly, transduction of retinal ganglion cells with AAV.ULK1.DN increased survival after optic nerve transection in 
vivo (Ribas et al. 2021). There is also conflicting evidence in the wider literature regarding the role of ULK1 in cell survival. For instance, one study demonstrated increased cell death following treatment of the cancer cell line MDA-MB-231 with the ULK1 agonist LYN-1604, which was reversed by simultaneous transfection with an siRNA against ULK1 (Zhang et al. 2017). In disagreement, application of the small-molecule ULK1 inhibitor SBI-0206965 onto A549 cells induced cell death, particularly when rapamycin was administered concurrently (Egan et al. 2015). Therefore, modulation of ULK1-dependent autophagy seems to exert different effects on cell survival depending on the context and model, warranting further studies to provide clarification.

\subsection{AAV.ULK1.DN attenuates axonal degeneration in vitro}

Having demonstrated a significant reduction of rapamycin-induced autophagy, it was speculated that AAV.ULK1.DN might also lead to a beneficial effect on autophagy induction after axonal injury and potentially counteract AAD. Indeed, the formation of degeneration bulbs was significantly attenuated for up to six hours after selective axonal lesion to primary cortical neurons cultured in microfluidic chambers, indicating that AAV.ULK1.DN attenuates AAD. Yet, it needs to be pointed out that, even though degeneration bulbs represent a hallmark of AAD (Coleman 2005), their formation is mostly an indirect measure for $\mathrm{AAD}$, as $\mathrm{AAD}$ is classically assessed by the grade of axon fragmentation quantified using the axonal integrity ratio (Knoferle et al. 2010). Axonal fragmentation, however, is not observed after injury to axons cultured in microfluidic chambers (Zhang et al. 2016), which is likely due to the fact that the surrounding microgrooves provide structural support, thereby preventing axons from disintegrating. The finding of beneficial effects of AAV.ULK1.DN on $\mathrm{AAD}$ in vitro demonstrated in this thesis thus needed further confirmation by in vivo experiments. Indeed, ONC experiments, which allow for the live-imaging of AAD in vivo (Koch et al. 2011), demonstrated attenuated AAD after both transduction of retinal ganglion cells with AAV.ULK1.DN and treatment with the small-molecule ULK1 inhibitor SBI0206965 (Vahsen et al. 2020). These results are also in line with the previously reported attenuation of AAD after ONC by unspecific autophagy inhibition using 3-MA (Knoferle et al. 2010). In addition, these findings were extended by the evaluation of long-term degeneration of axons of rubrospinal neurons one week after SCI, which also showed attenuated axonal degeneration rostral to the lesion in AAV.ULK1.DN-injected animals (Vahsen et al. 2020). Moreover, an axon-stabilizing effect of transduction with 
AAV.ULK1.DN was visible in axons of the corticospinal tract even five weeks after SCI (Ribas et al. 2021).

To date, a beneficial role of ULK1 inhibition on axon degeneration has not yet been described. The literature available is scarce, but there is some conflicting evidence from animal models describing that full Ulk1 knock-out leads to perinatal death and conditional Ulk1 knock-out induces axon guidance defects (Joo et al. 2016; Wang B et al. 2018). However, given that complete ULK1 depletion will severely disrupt neuronal homeostasis and is thus a highly different approach from the moderate inhibition of ULK1 performed in this thesis, these findings are only seemingly contradictory. Instead, they emphasize the importance of a moderate ULK1 inhibition for the generation of therapeutically beneficial effects (Vahsen and Lingor 2021).

To complete the analysis of axonal degeneration in vitro, the evaluation of WD would have been required after transduction with AAV.ULK1.DN. However, this is technically impossible in the microfluidic chambers employed in this thesis, as the distal part of the axon is aspirated during the lesion process. Therefore, the effect of AAV.ULK1.DN on WD was analyzed in the SCI model (Vahsen et al. 2020). The results showed no relevant axonprotective effect of AAV.ULK1.DN on WD, indicating that the modulation of ULK1dependent autophagy only elicits beneficial effects on axonal degeneration proximal to a lesion site. This observation could point to a beneficial role of AAV.ULK1.DN in axonprotective processes that require an intact connection between the axonal lesion area and the neuronal cell body, such as axonal transport or protein biosynthesis, which is in line with the molecular mechanisms of AAV.ULK1.DN delineated in this thesis (for details, see 4.6). Nonetheless, the protection of the axon part proximal to the injury might be sufficient to enable the formation of new axonal connections post-lesion. For instance, the inhibition of AAD using calcium channel inhibitors led to axon regeneration beyond the lesion site after ONC (Ribas and Lingor 2016; Ribas et al. 2017). To assess if AAV.ULK1.DN-mediated neuroprotection might indeed affect axonal regeneration positively, the effect of AAV.ULK1.DN on neurite outgrowth and the regeneration of lesioned axons of cortical neurons was investigated next in this thesis.

\subsection{AAV.ULK1.DN exerts pro-regenerative effects in vitro}

Primary cortical neurons transduced with AAV.ULK1.DN showed significantly enhanced neurite outgrowth on the permissive substrate laminin, indicating that ULK1 inhibition stimulates the intrinsic growth capacity of neuronal extensions. This result is in keeping with 
a previous study (Loh et al. 2008), in which siRNA-mediated knockdown of ULK1 increased neurite outgrowth of primary cerebellar granule neurons. Furthermore, Loh et al. (2008) demonstrated a strong increase in the regenerative response of mechanically lesioned neurites of primary dopaminergic neurons. In line with this, AAV.ULK1.DN significantly enhanced the axon regeneration of lesioned cortical neurons up to $96 \mathrm{~h}$ after axotomy in vitro, extending the aforementioned finding by demonstrating a specific effect on axons. To the author's best knowledge, a pro-regenerative effect of ULK1 inhibition on axon regeneration has not yet been shown. Furthermore, in addition to an enhanced intrinsic axonal growth capacity, AAV.ULK1.DN also significantly increased neurite outgrowth on the non-permissive substrate CSPG. This finding is of particular relevance, as regeneration after axonal lesion in vivo is severely hampered by both low intrinsic axon growth and inhibitory environmental signaling, both of which can be overcome by transduction of primary cortical neurons with AAV.ULK1.DN. Nonetheless, these in vitro findings called for validation experiments in vivo, as the complex pathophysiological events after axonal injury can only be fully replicated in animal models. Confirming the results of the in vitro experiments in this thesis, increased regenerative growth of retinal ganglion cell axons was observed four weeks after ONC in AAV.ULK1.DN-injected animals (Ribas et al. 2021). However, no pro-regenerative effects of AAV.ULK1.DN could be found five weeks after SCI in axons of the corticospinal tract (Ribas et al. 2021). Therefore, further experiments with additional animal models of axonal injury will be required to provide clarification regarding the pro-regenerative effect of ULK1 inhibition post-lesion in vivo. It might be possible that a sustained regenerative response in vivo requires the concomitant modulation of other key pathways of axonal regeneration, for example, the PTEN-PI3K-mTOR axis (for details, see 1.2.3).

\subsection{Understanding the molecular mechanisms of axon protection and axonal regeneration by AAV.ULK1.DN}

\subsubsection{Are the effects of AAV.ULK1.DN explained by autophagy inhibition?}

This entire project was initially based on the hypothesis that ULK1-mediated autophagy induction might represent a deleterious mediator of axonal degeneration post-injury. As a proof of concept, it was demonstrated in this thesis that AAV.ULK1.DN significantly inhibits autophagy in vitro, but it was not directly shown that the post-axotomy attenuation of AAD observed by AAV.ULK1.DN was mediated by an inhibition of autophagy. To establish a causal link would have been very technically challenging in the microfluidic chambers employed and was beyond the scope of this thesis. However, complementary in 
vivo-experiments demonstrated that autophagy levels after injury were significantly decreased in the rubrospinal tract of AAV.ULK1.DN-injected animals after SCI and, similarly, a reduction in autophagy was seen in lesioned retinal ganglion cells after ONC by treatment with the small-molecule ULK1 inhibitor SBI-0206965 (Vahsen et al. 2020). These findings are in line with the previously described attenuation of AAD after ONC by administration of the unspecific autophagy blocker 3-MA (Knoferle et al. 2010). Furthermore, extended axon elongation was observed after autophagy inhibition by siRNA against $\operatorname{Atg} 7$ (Ban et al. 2013). However, there is a plethora of evidence showing beneficial and detrimental consequences of both autophagy inhibition and activation in models of axonal degeneration and regeneration. For instance, in contrast to the findings in this thesis, cytoprotective roles of autophagy and autophagy-inducing drugs have been described in models of acute axonal injury, such as optic nerve transection and traumatic brain injury (Erlich et al. 2007; Rodriguez-Muela et al. 2012). Similarly, rapamycin-mediated autophagy induction was found to promote axon regeneration after axonal injury in C. elegans by limiting Notch signaling (Ko et al. 2020), and this was also seen with the administration of the autophagy-inducing peptide Tat-beclin1, which promoted the regeneration of descending spinal cord axons after mouse SCI (He et al. 2016). It therefore seemed likely that reduced autophagy by AAV.ULK1.DN might be one molecular mechanism responsible for its beneficial effects, but that the regulation of additional processes might also be involved. Hence, a comprehensive assessment of the underlying molecular mechanisms was performed by transcriptomic and proteomic analyses in order to better understand the demonstrated axon-protective and proregenerative properties of AAV.ULK1.DN in an unbiased approach. Supporting a key involvement of further processes in addition to autophagy, neither transcriptomics nor proteomics outlined any regulation of genes or proteins with direct involvement in the autophagy cascade. Instead, a distinct regulation of proteins involved in translation and splicing was uncovered in primary neurons transduced with AAV.ULK1.DN.

\subsubsection{AAV.ULK1.DN mediates the differential splicing of axon degeneration and regeneration-associated genes}

The effect of AAV.ULK1.DN on splicing-associated proteins demonstrated in this study was a very surprising result and unraveled a previously unknown and novel function of ULK1 in splicing. This finding was further extended by the transcriptomic analysis of differential exon usage, demonstrating that AAV.ULK1.DN modulates the alternative splicing of 36 genes. Alternative splicing is a highly abundant process in the brain characterized by the different combination of exons from one gene to various mRNA transcripts, allowing for a 
single gene to encode multiple different protein isoforms with potentially different or even antagonistic functions (Licatalosi and Darnell 2006). The discovery of differential splicing after ULK1 inhibition is especially intriguing, as axonal splicing is of importance for axon growth (Zhang et al. 2019; Zheng 2020), and the dysregulation of alternative splicing is a well-known feature of many neurodegenerative disorders, particularly ALS and spinal muscular atrophy (SMA) (Eggert et al. 2006; Nik and Bowman 2019; Orozco and Edbauer 2013). In SMA patients, the levels of the survival motor neuron (SMN) protein are significantly reduced due to loss-of-function mutations in the SMN1 gene, and the second copy of the gene, $S M N 2$, cannot compensate for this, as it mostly generates an unstable protein variant owing to a naturally occurring exon-skipping mutation in exon 7 . In turn, low SMN levels result in extensive splicing alterations due to its function as an RNA-binding protein, for instance, of critical mediators of axon guidance and homeostasis, which ultimately leads to motor neuron death (Nik and Bowman 2019; Pellizzoni et al. 1998). Antisense oligonucleotide therapy is able to repress exon 7 splicing in SMN2, allowing for the sufficient production of the full-length SMN protein (Rigo et al. 2012), and has proven successful in infants with SMA (Finkel et al. 2017), emphasizing the importance of alternative splicing in neurodegeneration. Similarly, multiple ALS-related genes encode RNA-binding proteins. For example, TARDP encoding for transactive response DNA-binding protein of $43 \mathrm{kDa}$ (TDP-43) is widely implicated in the regulation of alternative splicing, and TARDP mutations and cytoplasmic mislocalization of TDP-43 are thought to cause motor neuron degeneration through splicing misregulation (Butti and Patten 2018; Nik and Bowman 2019; Prasad et al. 2019).

Supporting the biological relevance of the splicing events by AAV.ULK1.DN, an enrichment analysis of functional annotations to the differentially spliced genes outlined a clear role in axon degeneration and regeneration-associated mechanisms. Particularly, microtubule and axonal transport-associated genes showed alternative splicing by AAV.ULK1.DN.

\subsubsection{Differential splicing of Kiflb might mediate the beneficial effects of AAV.ULK1.DN via a non-canonical modulation of axonal transport}

One of the genes that especially stood out in the differential exon expression analysis was Kif1b, which encodes for an axonal transport motor protein. Kif1b is of particular interest, because its reduced retinal expression was connected to retinal ganglion cell demise in a mouse model of chronic glaucoma, and it was also described to be mutated in the neuropathy Charcot-Marie-Tooth disease type 2A (Breen et al. 2016; Zhao et al. 2001). Very interestingly, in a Drosophila model, ULK1 has already been connected to kinesin 1-dependent axonal 
transport, and alternative splice forms of Kiflb were shown to induce higher activity and affinity for microtubules in vitro (Matsushita et al. 2009; Toda et al. 2008). The results in this thesis therefore establish a functional link between ULK1 and differential splicing of Kif1b, demonstrating increased exon usage of Kif1 $b$ after transduction with AAV.ULK1.DN. These data suggest that AAV.ULK1.DN might additionally exert its axon-protective through enhanced or modulated axonal transport via regulation of Kif1b. The experimental confirmation that ULK1 inhibition directly affects axonal transport after injury was beyond the scope of this work, but it should be performed in future experiments. For example, this assessment could be done by treating transduced neurons cultured in microfluidic chambers with the mitochondrial stain MitoTracker, followed by the analysis of axonal transport using a live-imaging microscope. Provided a modulation of axonal transport would be observed by AAV.ULK1.DN, it should be validated in a next step that it is indeed differential splicing of Kif1b that is responsible for this effect. To do so, one could transduce neurons with AAV.ULK1.DN and perform an additional knock-down or knock-out of Kiftb. Alternatively, one could rescue the differential expression of the Kif1b exons using the clustered regularly interspaced short palindromic repeats(CRISPR)-Cas9 technique to investigate whether this abolishes the neuroprotective properties of ULK1 inhibition or alters its putative effect on axonal transport.

\subsubsection{AAV.ULK1.DN leads to the differential splicing of Ddit3 and might protect from ER stress after axonal injury}

In addition, differential exon expression of Ddit 3 was demonstrated after transduction with AAV.ULK1.DN in this thesis. This finding is of special interest, because Ddit3 is a mediator of ER stress, which is increasingly being implicated in neurodegeneration (Hu 2016; Li et al. 2013). Furthermore, it was recently demonstrated that ULK1 mediates the autophagyinduced inhibition of ER-to-Golgi traffic (Gan et al. 2017). Ddit3 was shown to be upregulated in neurons after mouse SCI, and its knock-out improved functional recovery after lesion (Ohri et al. 2011). Similarly, Ddit3 levels were increased after optic nerve injury, while its deletion improved retinal ganglion cell survival (Hu et al. 2012). Interestingly, AAV.ULK1.DN reduced the differential exon expression of Ddit3, suggesting that attenuated ER stress might be another means by which ULK1 inhibition protects from degeneration. However, it is important to note that this finding is preliminary and warrants further investigation. In order to test this hypothesis, transduced neurons could be treated with the widely employed ER stress inducer thapsigargin to assess whether AAV.ULK1.DN protects from ER stress. If this were the case, experimental rescue of the modulated splicing 
of $D$ dit 3 should be performed in transduced neurons to confirm that it indeed represents a molecular mediator of the effects of AAV.ULK1.DN.

In addition to its role in ER stress, Ddit3 also acts as an pro-apoptotic transcription factor (Yang et al. 2017). While the absence of an effect on cell survival by AAV.ULK1.DN in primary neuron cultures renders it unlikely that the differential exon expression of Ddit3 elicits axon-protective effects through a modulation of apoptosis in vitro, differential splicing of $D$ dit 3 could potentially explain some of the pro-survival effects of AAV.ULK1.DN seen in vivo (for details, see 4.3). Future studies should therefore assess the role of Ddit3 in cell survival in AAV.ULK1.DN-transduced animals in more detail.

\subsubsection{Translation is enhanced by AAV.ULK1.DN via an mTOR-S6-dependent and PTEN-AKT-GSK3 $\beta$-independent mechanism}

In addition to a regulation of proteins involved in splicing, a second major hit in the proteomic analysis was a distinct upregulation of translation-associated proteins after transduction with AAV.ULK1.DN. This finding was characterized in more detail by multiple Western blots against the translational master regulator mTOR and its associated pathway, demonstrating that AAV.ULK1.DN stimulates translation by upregulating the expression of active (phosphorylated) mTOR. Increased levels of p-S6, a downstream target of mTOR that is of crucial importance for the initiation of translation (Dufner and Thomas 1999), confirmed this result. The complementary assessment of the translational repressor 4E-BP1 showed no differences after transduction with AAV.ULK1 (Vahsen et al. 2020), demonstrating that increased translation is mediated via an 4E-BP1-independent mechanism. To assess the modulation of the mTOR pathway more widely after transduction with AAV.ULK1.DN, the upstream regulators of mTOR AMPK, PTEN, AKT, and GSK3 $\beta$ were additionally analyzed.

Reduced levels of active (phosphorylated) AMPK $\alpha$, an inhibitor of mTOR (Alers et al. 2012a), were demonstrated after transduction with AAV.ULK1.DN. While reduced AMPK $\alpha$ activity corresponds well with the finding of increased mTOR activation, it is in contrast to a previously described mechanism of ULK1-mediated AMPK inhibition (Loffler et al. 2011). Furthermore, application of the ULK1 inhibitor SBI-0206965 led to the opposing results of increased p-AMPK $\alpha$ expression, albeit in a much more acute paradigm (Vahsen et al. 2020). Therefore, the detailed effect of ULK1 inhibition on AMPK currently remains uncertain and should be assessed in future experiments in more detail, including the analysis of the additional AMPK subunits AMPK $\beta$ and AMPK $\gamma$. 
The analysis of total and phosphorylated PTEN and GSK3 $\beta$ showed no differential expression after transduction with AAV.ULK1.DN. Similarly, AKT levels remained unchanged, but AAV.ULK1.DN led to significantly lower levels of active (phosphorylated) AKT. Reduced p-AKT levels were also reported after Ulk1 knock-out by Jung et al. (2011), and they are likely due to a negative feedback loop between S6 and AKT signaling (Tremblay et al. 2007; Tzatsos and Kandror 2006; Zhang et al. 2008).

In combination, these findings suggest that AAV.ULK1.DN-mediated ULK1 inhibition stimulates mTOR in a direct mechanism that is independent of upstream regulators, with some uncertainty towards the role of AMPK. This observation is in keeping with a detailed study on the effect of Ulk1 knock-out on mTOR signaling in mouse embryonic fibroblasts and 293T cells, in which a direct effect of ULK1 on mTORC1 was postulated independent of upstream kinases (Jung et al. 2011); however, it should be noted that AMPK levels were not addressed in this study. The results in this thesis therefore corroborate the existence of a direct autophagy-translation switch governed by mutual inhibition between ULK1 and mTORC1. This interaction has been proposed by computational models (Szymanska et al. 2015) and has been confirmed in detail by experimental evidence (Alers et al. 2012a; Alers et al. 2012b; Jung et al. 2011; Jung et al. 2009). The increased mTOR activity observed in this study is therefore not a surprising finding after AAV.ULK1.DN-mediated ULK1 inhibition. Elevated levels of p-mTOR were also observed in mouse dopaminergic neurons after injection of AAV.ULK1.DN into the midbrain (Balke et al. 2020) and after treatment of primary cortical neurons with the ULK1 inhibitor SBI-0206965 (Vahsen et al. 2020), confirming the results demonstrated in this thesis.

Regarding the functional implication of the increased translation by AAV.ULK1.DN described here, it is important to consider that translation is not only key for neuronal homeostasis in general, but it is also particularly relevant for axonal maintenance (Shigeoka et al. 2016; Spaulding and Burgess 2017). Furthermore, recent evidence has underlined the importance of local axonal translation by pointing out the implication of dysregulated translation in axonal degeneration and regeneration processes (Costa and Willis 2018). Amongst others, specific deficits in local translation were observed in the neurodegenerative disorders SMA and ALS, connected to cytoskeletal defects and dysfunctional axonal transport (Costa and Willis 2018; Sahoo et al. 2018; Spaulding and Burgess 2017). A potential means by which AAV.ULK1.DN protects from axon degeneration and supports axonal regeneration might therefore be an increased translation of traffic molecules, which transport molecules that support axonal health or a direct local synthesis of axon-protective and pro- 
regenerative factors. This remains speculative at this stage, as the proteomics and Western blot analyses were performed using whole-cell lysates from cultured cortical neurons. Even though the relative maturity of the cultures analyzed (8 DIV) ensures that a significant proportion of the proteome will originate from the axons, further studies will be required to provide clarification that it is indeed local translation in the axon that is modulated by AAV.ULK1.DN. A useful technique to employ would be the recently developed translating ribosome affinity purification (TRAP) (Heiman et al. 2008; Shigeoka et al. 2016), which could allow for the detailed analysis of the axonal translatome in response to ULK1 inhibition and thus shed more light on the underlying mechanisms.

\subsubsection{AAV.ULK1.DN stimulates the intrinsic axonal growth capacity via increased ERK1 activation independent of JNK and STAT3 signaling}

An additional gene found to be differentially spliced by AAV.ULK1.DN here was Mapkap3. MAPK signaling is well known to be implicated in cell growth and axon regeneration (Raivich and Makwana 2007) and was therefore investigated in more detail. Increased levels of active (phosphorylated) ERK1 were seen after transduction with AAV.ULK1.DN, indicating that enhanced MAPK signaling contributes to its pro-regenerative effects. The levels of the other isoform, p-ERK2, were not significantly altered, and neither was the expression of another MAPK termed JNK, arguing for a more specific interaction between AAV.ULK1.DN and ERK1.

Very little has been reported in the literature about an interaction between ULK1 and ERK. One study (Egan et al. 2015) showed a dose-dependent elevation of both p-ERK1/2 isoforms after application of increasing doses of the ULK1 inhibitor SBI-0206965 by Western blotting, but it failed to demonstrate a quantification of these results. Yet, there also seemed to be a preferential effect on p-ERK1 expression (Egan et al. 2015). On the other hand, a direct interaction between ATG proteins and ERK has been described, with ERK proteins localizing to the extraluminal membrane of autophagosomes and their phosphorylation being dependent on ATG5, ATG7, and LC3 (Martinez-Lopez et al. 2013). However, the levels of ATG5 and ATG7 were not altered by AAV.ULK1.DN in this study, and there was no difference in p-ERK1 levels between naïve and autophagy-inducing conditions, suggesting that the increase in p-ERK1 expression might rather be a direct effect of ULK1 inhibition than due to a regulation of autophagy.

Increased ERK1 activation corresponds well to the activation of the mTOR pathway by AAV.ULK1.DN, as there is considerable cross-talk with MAPK signaling leading to mTOR 
activation (Mendoza et al. 2011). Furthermore, it is an interesting finding that there appears to be a specific effect of AAV.ULK1.DN only on the ERK1 isoform. Both ERK isoforms were found to be ubiquitously expressed and share functional redundancy (Busca et al. 2016). However, very intriguingly, ERK2 was described to mediate secondary injury mechanisms and neurological dysfunction, while ERK1 is deemed to antagonize ERK2 signaling and provide neuroprotection in the CNS (Yu 2012). Activated ERK1 supports the formation of a new growth cone and axon elongation via the regulation of a vast array of transcription factors involved in cellular growth (Chierzi et al. 2005; Raivich and Makwana 2007). To complement this finding, the levels of STAT3, another key regulator of genes required for axonal regeneration, were also analyzed in this study. However, STAT3 levels remained unchanged after transduction with AAV.ULK1.DN, while the expression of its active (phosphorylated) form decreased. This finding is in line with a previous report on the effect of siRNA-mediated knockdown of ULK1 (Cho et al. 2017), indicating that only ERK1mediated activation of growth-associated transcription factors represents a molecular mechanism by which AAV.ULK1.DN supports axonal regeneration after injury. Further studies will be required to understand the mechanism of how exactly AAV.ULK1.DN leads to the activation of ERK1 in detail.

\subsubsection{Inhibitory environmental signaling after lesion is counteracted by AAV.ULK1.DN via downregulation of ROCK2}

In addition to an increased intrinsic growth capacity, transduction with AAV.ULK1.DN counteracted the growth-inhibiting effects of CSPG. Mechanistically, CSPG activates RhoAROCK2 signaling, resulting in impaired growth and the promotion of axon degeneration (Koch et al. 2014a; Koch et al. 2014b). AAV.ULK1.DN led to reduced exon usage of the CSPG-signaling related gene Ptprf and significantly lower ROCK2 expression, explaining the observed rescue of CSPG-induced growth impairment on a molecular level. In keeping, AAV.ULK1.DN also reduced the levels of RhoA (Ribas et al. 2021). Several studies in the laboratory previously demonstrated beneficial effects of ROCK2 downregulation and inhibition, attenuating axonal degeneration, and fostering axon regeneration and growth (Challagundla et al. 2015; Koch et al. 2014a; Koch et al. 2014b; Lingor et al. 2007). However, to the author's best knowledge, an interaction between ULK1 and ROCK2 has not yet been demonstrated. Intriguingly, it was previously shown that siRNA-mediated inhibition of $\operatorname{Atg} 7$ led to increased axon elongation via reduced RhoA expression (Ban et al. 2013). Ban et al. (2013) suggested that the heterogeneous nuclear ribonucleoprotein Q1 (hnRNP-Q1) might be an autophagy substrate accumulating after Atg7 knock-down, thereby inhibiting RhoA- 
ROCK2 signaling. A further characterization of this interaction after transduction with AAV.ULK1.DN was beyond the scope of this study but should be performed in future experiments. However, it also seems plausible that decreased ROCK2 expression might be a direct effect of ULK1 inhibition, as lower ROCK2 levels by AAV.ULK1.DN were also observed in naïve culture conditions, where conventional autophagy was not significantly regulated.

In an attempt to understand the downstream signaling events of ROCK2, the levels of CRMP2 were studied after transduction with AAV.ULK1.DN, but CRMP2 levels were not significantly regulated. This is a slightly surprising finding, given that multiple microtubuleassociated genes were regulated by AAV.ULK1.DN in the differential expression analysis. However, CRMP2 only represents one of a vast array of downstream targets of ROCK2 (Koch et al. 2018), the expression of which remains to be characterized after transduction with AAV.ULK1.DN. Other relevant targets that could be interesting to analyze would be LIMK and cofilin (Koch et al. 2018).

\subsection{AAV.ULK1.DN mediates axon protection and regeneration through a molecular switch from autophagy to axon-protective and growth-promoting pathways}

Originally, this project was based on the previous finding of ULK1 and other autophagic proteins accumulating after axonal lesion (Ribas et al. 2015), leading to the hypothesis that ULK1-dependent autophagy might be a deleterious executing mechanism during axonal degeneration (Figure 38A). Indeed, this thesis has demonstrated that inhibition of ULK1 function using AAV.ULK1.DN protects from axonal degeneration and enhances axonal regeneration after axonal injury in vitro (Figure 38B), confirming a detrimental role of ULK1 in the biochemical cascade after axonal injury. However, contrary to initial speculation, the combined evidence presented in this thesis has uncovered that the role of ULK1 in neuron biology goes far beyond its autophagy-initiating function. Comprehensive analyses have elucidated that AAV.ULK1.DN-mediated ULK1 inhibition leads to a molecular switch from the catabolic autophagy pathway to the advantageous activation of axon-protective and axon growth-promoting processes (Figure 38B). These beneficial processes comprise mTORdependent translation, altered ROCK2 and ERK1 signaling, which are known to foster actin dynamics and growth-associated gene expression, and the differential splicing of genes such as Kif1 $b$ and Ddit3, which could mediate axon protection through increased axonal transport and reduced ER stress, respectively. 
A

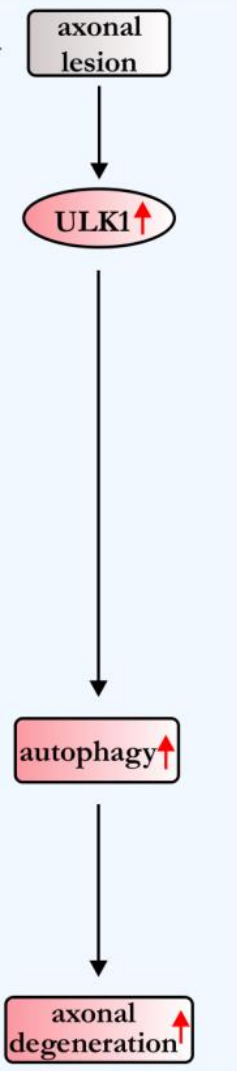

B

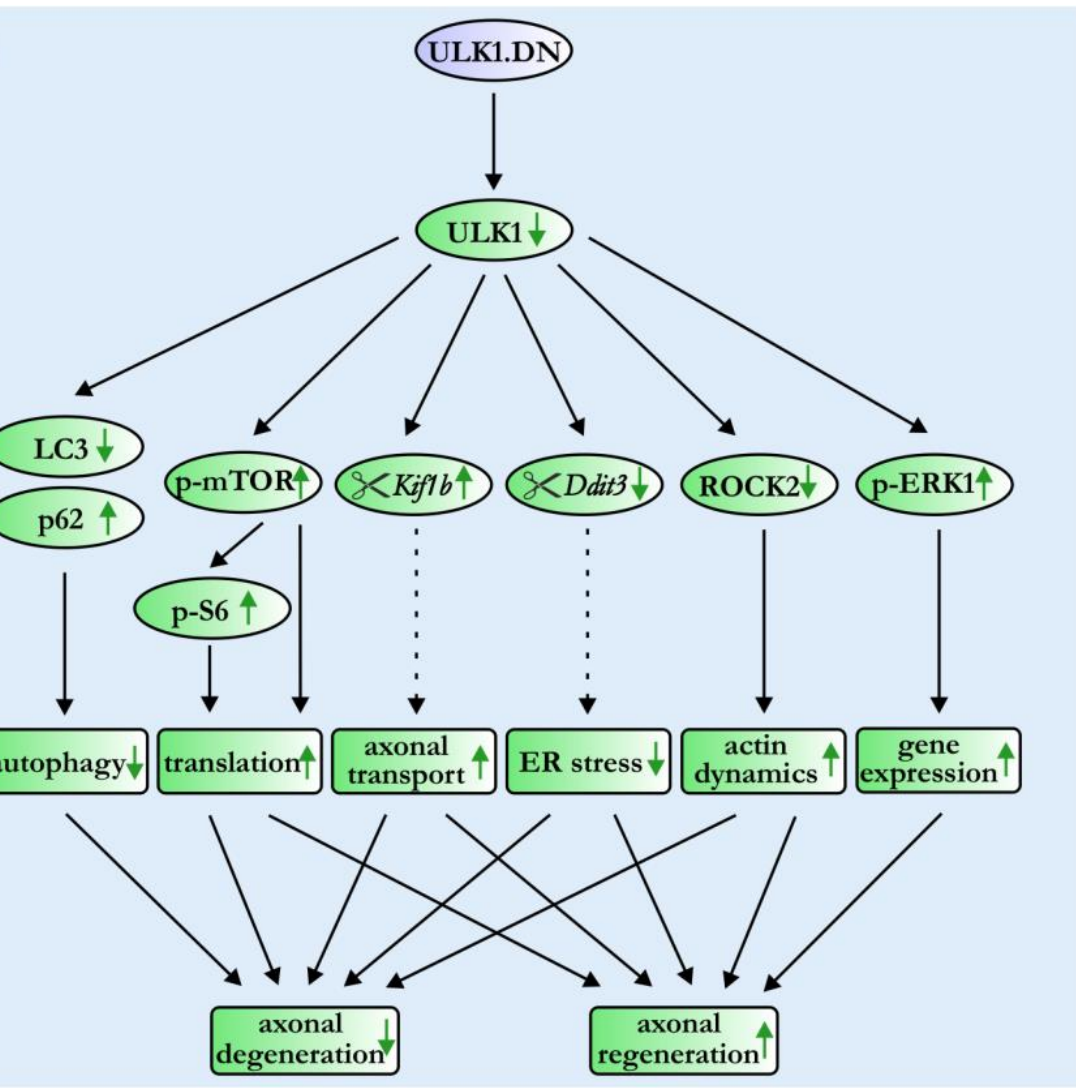

Figure 38: Summary of the findings on the role of ULK1 in axonal degeneration and regeneration in this thesis. (A) It was previously demonstrated that ULK1-mediated autophagy induction is evident during axonal degeneration after axonal injury, leading to the hypothesis that ULK1-dependent autophagy is a detrimental mechanism executing axonal degeneration post-injury. (B) In this thesis, AAV.ULK1.DNmediated inhibition of ULK1 function protected axons from degeneration and promoted axonal regeneration in vitro. These findings were connected to reduced autophagy, increased mTOR-dependent translation, differential splicing of the genes Kifl b and Ddit3, reduced expression of ROCK2, and increased levels of active ERK1. While altered ROCK2 and ERK1 levels are known to improve actin dynamics and induce growthassociated gene expression, the differential splicing of Kifl $b$ and Ddit3 might lead to enhanced axonal transport and reduced ER stress. Together, AAV.ULK1.DN-mediated ULK1 inhibition therefore mediates a molecular switch from autophagy to axon-protective and growth-promoting pathways. Figure adapted from Vahsen et al. (2020) and Ribas et al. (2021), both licensed under a CC BY 4.0 license, https://creativecommons.org/licenses/by/4.0/.

These findings therefore lead to a model in which ULK1 is a master regulator of neuron biology, governing not only autophagy, but also translation, splicing, and growth-associated pathways. While a fine-tuned balance between these diverse functions is probably crucial to maintain neuronal homeostasis, its dysregulation after axonal injury appears to promote selfdigestion of the axon and inhibit its perseverance and re-growth. Targeted inhibition of ULK1 by AAV.ULK1.DN rescues this dysregulation, unraveling ULK1 as a novel target to overcome degeneration of CNS axons after lesion and promote their regeneration (Figure 38B). 


\subsection{Outlook and clinical relevance}

The beneficial effects of ULK1 inhibition in vitro demonstrated in this thesis pave the way for a wider and more thorough experimental investigation of ULK1 inhibition in axonal degeneration and regeneration. It is important to consider that the findings in this thesis were obtained using primary rat neurons and thus their relevance for human disease will need to be further validated.

To do so, induced pluripotent stem cell (iPSC) models (Takahashi and Yamanaka 2006) represent a promising method to confirm the relevance of ULK1 inhibition in in vitro paradigms with human cells. Differentiation protocols are currently available for a plethora of different cell types, including multiple subtypes of neurons, and allow for large-scale and high-throughput experiments (Ebert et al. 2012). Furthermore, the possibility to generate iPSCs from patients with neurodegenerative diseases facilitates future studies on the therapeutic effects of ULK1 inhibition in the wider context of these disorders, as the differentiated cells maintain their genetic background (Ebert et al. 2012). Intriguingly, iPSC co-culture models of neurons with additional cell types, such as oligodendrocytes (Clark et al. 2017), astrocytes (di Domenico et al. 2019), and microglia (Haenseler et al. 2017), have recently been developed. These models are of particular importance, as neuronal monocultures cannot reflect and model the involvement of glia cells in degenerative processes. Therefore, it would be important to investigate the effect of ULK1 inhibition during axon degeneration and regeneration in cultures with multiple cell types. Given the homeostatic importance of autophagy, it should also be carefully evaluated if ULK1 inhibition results in relevant toxicity in non-neuronal cells.

Additionally, further animal models of disease should be harnessed that allow for a more accurate replication of the pathophysiological processes of neurodegeneration than in vitro paradigms. Protective effects of AAV.ULK1.DN-mediated ULK1 inhibition have already been shown in rat models of ONC and SCI (Vahsen et al. 2020; Ribas et al. 2021), as well as in a mouse model of PD (Balke et al. 2020). Hence, additional models of disease should be employed to investigate whether the demonstrated findings are transferable to other neurological disorders such as ALS. A good candidate would be the ALS mouse model expressing mutant superoxide dismutase 1 (SOD1 ${ }^{\mathrm{G} 93 \mathrm{~A}}$ ), in which a relevant involvement of autophagy in the pathophysiological cascade was already demonstrated (Rudnick et al. 2017). With regards to translational applicability, it will be key to substitute AAV-mediated ULK1 inhibition with a therapeutically more feasible approach. Some of the key findings of AAVmediated ULK1 inhibition were already validated using the small-molecule ULK1 inhibitor 
SBI-0206965 in vitro and in vivo (Vahsen et al. 2020). Future experiments should therefore aim to evaluate the beneficial effects of SBI-0206965-mediated ULK1 inhibition in additional animal models and also to assess its dose-response curves and potential side effects (Vahsen and Lingor 2021). Connected with this, it should be investigated whether the combination of ULK1 inhibition together with additional targeted strategies could potentiate therapeutic effects, bearing in mind that this might also lead to increased toxicity. For example, a potential combinatorial approach might be the concomitant inhibition of ULK1 and ROCK2, as the ROCK inhibitor Fasudil elicited beneficial effects in various experimental models of degeneration and regeneration (Koch et al. 2018), and is currently in a phase IIa clinical trial for the treatment of ALS (Koch et al. 2020; Lingor et al. 2019).

In summary, the findings in this thesis raise profound interest in further and detailed evaluation of ULK1 inhibition in traumatic and degenerative disorders of the CNS. Additional positive results and beneficial effects of ULK1 inhibition in pre-clinical models could lay the foundation for the clinical testing of ULK1 inhibitors and, ultimately, the therapeutic modulation of ULK1 in humans.

\subsection{Conclusion}

In conclusion, this study has demonstrated that AAV.ULK1.DN-mediated ULK1 inhibition enhances neurite outgrowth, attenuates axonal degeneration, and fosters axonal regeneration after traumatic injury to primary cortical neurons in vitro. Mechanistically, ULK1 inhibition leads to a protective molecular switch from autophagy to enhanced mTOR-dependent translation, activated growth-promoting signaling via ERK1 activation and ROCK2 downregulation, and the differential splicing of degeneration and regeneration-associated genes such as Kif1b and Ddit3. These findings uncover ULK1 as a key mediator of axonal degeneration and outline a much more multifaceted role in neuronal biology than previously known, including a novel function of ULK1 in splicing. ULK1 therefore represents a putative therapeutic target in traumatic and neurodegenerative disorders of the CNS. 


\section{Summary}

In many traumatic and neurodegenerative disorders of the central nervous system, axonal degeneration is a central and early pathophysiological feature. Since the regenerative response of injured neurons is severely hampered by an intrinsically reduced growth capacity and external growth-inhibiting factors such as CSPG, axonal degeneration often results in progressive clinical disability. An improved understanding of the molecular mechanisms is hoped to unravel potential therapeutic targets, as there are currently no therapeutic options available. During axonal degeneration, macroautophagy (here: autophagy), a cellular homeostatic process responsible for the degradation of long-lived organelles and proteins, was previously shown to be activated, but its detailed role in this context, harmful or beneficial, is incompletely understood. Particularly, the autophagy-initiating kinase ULK1 accumulated in degenerating axons, leading to the speculation that ULK1-dependent autophagy might represent a deleterious executing process during axonal degeneration.

To assess this hypothesis in the present study, primary cortical neurons were transduced with an adeno-associated viral vector expressing kinase-dead dominant-negative ULK1, leading to the inhibition of ULK1 function. After selective axonal lesion to transduced neurons cultured in microfluidic chambers, dominant-negative ULK1 attenuated acute axonal degeneration for up to six hours and fostered axonal regeneration up to 96 hours post-injury, as compared with control. Correspondingly, increased neurite outgrowth was observed after transduction with dominant-negative ULK1 in neurons cultured on both the permissive substrate laminin and the growth-inhibiting matrix CSPG. Mechanistically, dominantnegative ULK1 reduced autophagy activation, indicating that a decrease in autophagy is one mechanism underlying its axon-protective effects. The additional proteomic analysis of neurons transduced with dominant-negative ULK1 surprisingly outlined a strong regulation of splicing and translation-associated proteins. This finding was corroborated by a transcriptomic analysis uncovering dominant-negative ULK1-dependent differential splicing of axonal degeneration and regeneration-associated genes such as Kiflb and Ddit3. Dominant-negative ULK1 might therefore additionally elicit axon-protective and proregenerative properties via a modulation of Kif1b-dependent axonal transport and Ddit3mediated endoplasmic reticulum stress. Furthermore, the beneficial effects of dominantnegative ULK1 on axonal degeneration and regeneration were connected to an activation of mTOR-S6-mediated translation, elevated expression of the growth-promoting molecule pERK1, and reduced levels of the degeneration-mediator and growth-inhibitor ROCK2. 
Together, the data obtained in this thesis reveal a key involvement of ULK1 in axonal degeneration and regeneration in vitro, and demonstrate a more complex role of ULK1 in neuronal biology than previously known, including a novel function in splicing. ULK1 inhibition attenuates the degeneration of axons and promotes their regeneration in vitro by a proposed switch from the catabolic autophagy process to the activation of axon-protective and axon growth-promoting processes. ULK1 inhibition thus represents a putative therapeutic approach in traumatic and neurodegenerative disorders of the central nervous system. 


\section{Bibliography}

Alers S, Loffler AS, Wesselborg S, Stork B (2012a): Role of AMPK-mTOR-Ulk1/2 in the regulation of autophagy: cross talk, shortcuts, and feedbacks. Mol Cell Biol $\underline{32}, 2-11$

Alers S, Loffler AS, Wesselborg S, Stork B (2012b): The incredible ULKs. Cell Commun Signal $\underline{10}, 7$

Ashburner M, Ball CA, Blake JA, Botstein D, Butler H, Cherry JM, Davis AP, Dolinski K, Dwight SS, Eppig JT, et al. (2000): Gene ontology: tool for the unification of biology. The Gene Ontology Consortium. Nat Genet 25, 25-29

Atanassov I, Urlaub H (2013): Increased proteome coverage by combining PAGE and peptide isoelectric focusing: comparative study of gel-based separation approaches. Proteomics $\underline{13}$, 2947-2955

Balke D, Tatenhorst L, Dambeck V, Ribas VT, Vahsen BF, Michel U, Bahr M, Lingor P (2020): AAV-mediated expression of dominant-negative ULK1 increases neuronal survival and enhances motor performance in the MPTP mouse model of Parkinson's disease. Mol Neurobiol $\underline{57}, 685-697$

Ban BK, Jun MH, Ryu HH, Jang DJ, Ahmad ST, Lee JA (2013): Autophagy negatively regulates early axon growth in cortical neurons. Mol Cell Biol $\underline{33}$, 3907-3919

Bar-Yosef T, Damri O, Agam G (2019): Dual role of autophagy in diseases of the central nervous system. Front Cell Neurosci 13, 196

Barros Ribeiro da Silva V, Porcionatto M, Toledo Ribas V (2020): The rise of molecules able to regenerate the central nervous system. J Med Chem $\underline{63}$, 490-511

Bisicchia E, Latini L, Cavallucci V, Sasso V, Nicolin V, Molinari M, D'Amelio M, Viscomi MT (2017): Autophagy inhibition favors survival of rubrospinal neurons after spinal cord hemisection. Mol Neurobiol $\underline{54}$, 4896-4907

Bjorkoy G, Lamark T, Brech A, Outzen H, Perander M, Overvatn A, Stenmark H, Johansen T (2005): p62/SQSTM1 forms protein aggregates degraded by autophagy and has a protective effect on huntingtin-induced cell death. J Cell Biol 171, 603-614

Breen KT, Anderson SR, Steele MR, Calkins DJ, Bosco A, Vetter ML (2016): Loss of fractalkine signaling exacerbates axon transport dysfunction in a chronic model of glaucoma. Front Neurosci 10, 526 
Burnette WN (1981): Western blotting - electrophoretic transfer of proteins from sodium dodecyl sulfate-polyacrylamide gels to unmodified nitrocellulose and radiographic detection with antibody and radioiodinated protein A. Anal Biochem 112, 195-203

Busca R, Pouyssegur J, Lenormand P (2016): ERK1 and ERK2 map kinases: specific roles or functional redundancy? Front Cell Dev Biol 4,53

Butti Z, Patten SA (2018): RNA dysregulation in amyotrophic lateral sclerosis. Front Genet $\underline{9}, 712$

Cargnello M, Roux PP (2011): Activation and function of the MAPKs and their substrates, the MAPK-activated protein kinases. Microbiol Mol Biol Rev $\underline{75}$, 50-83

Challagundla M, Koch JC, Ribas VT, Michel U, Kugler S, Ostendorf T, Bradke F, Muller HW, Bahr M, Lingor P (2015): AAV-mediated expression of BAG1 and ROCK2-shRNA promote neuronal survival and axonal sprouting in a rat model of rubrospinal tract injury. $\mathrm{J}$ Neurochem 134, 261-275

Chan EY, Kir S, Tooze SA (2007): siRNA screening of the kinome identifies ULK1 as a multidomain modulator of autophagy. J Biol Chem 282, 25464-25474

Chan EY, Longatti A, McKnight NC, Tooze SA (2009): Kinase-inactivated ULK proteins inhibit autophagy via their conserved C-terminal domains using an Atg13-independent mechanism. Mol Cell Biol 29, 157-171

Chierzi S, Ratto GM, Verma P, Fawcett JW (2005): The ability of axons to regenerate their growth cones depends on axonal type and age, and is regulated by calcium, cAMP and ERK. Eur J Neurosci 21, 2051-2062

Cho SW, Na W, Choi M, Kang SJ, Lee SG, Choi CY (2017): Autophagy inhibits cell death induced by the anti-cancer drug morusin. Am J Cancer Res $\underline{7}, 518-530$

Clark AJ, Kaller MS, Galino J, Willison HJ, Rinaldi S, Bennett DLH (2017): Co-cultures with stem cell-derived human sensory neurons reveal regulators of peripheral myelination. Brain $\underline{140}, 898-913$

Coleman M (2005): Axon degeneration mechanisms: commonality amid diversity. Nat Rev Neurosci $\underline{6}, 889-898$

Coleman MP, Hoke A (2020): Programmed axon degeneration: from mouse to mechanism to medicine. Nat Rev Neurosci 21, 183-196 
Conforti L, Gilley J, Coleman MP (2014): Wallerian degeneration: an emerging axon death pathway linking injury and disease. Nat Rev Neurosci 15, 394-409

Costa CJ, Willis DE (2018): To the end of the line: Axonal mRNA transport and local translation in health and neurodegenerative disease. Dev Neurobiol $\underline{78}$, 209-220

Crouch SPM, Kozlowski R, Slater KJ, Fletcher J (1993): The use of ATP bioluminescence as a measure of cell-proliferation and cytotoxicity. J Immunol Methods $\underline{160}, 81-88$

Cuervo AM, Wong E (2014): Chaperone-mediated autophagy: roles in disease and aging. Cell Res 24, 92-104

Curcio M, Bradke F (2018): Axon regeneration in the central nervous system: facing the challenges from the inside. Annu Rev Cell Dev Biol $\underline{34}$, 495-521

di Domenico A, Carola G, Calatayud C, Pons-Espinal M, Munoz JP, Richaud-Patin Y, Fernandez-Carasa I, Gut M, Faella A, Parameswaran J, et al. (2019): Patient-specific iPSCderived astrocytes contribute to non-cell-autonomous neurodegeneration in Parkinson's disease. Stem Cell Reports 12, 213-229

Di Stefano M, Nascimento-Ferreira I, Orsomando G, Mori V, Gilley J, Brown R, Janeckova L, Vargas ME, Worrell LA, Loreto A, et al. (2015): A rise in NAD precursor nicotinamide mononucleotide (NMN) after injury promotes axon degeneration. Cell Death Differ 22, 731 742

Ding WX, Ni HM, Gao W, Hou YF, Melan MA, Chen X, Stolz DB, Shao ZM, Yin XM (2007): Differential effects of endoplasmic reticulum stress-induced autophagy on cell survival. J Biol Chem 282, 4702-4710

Dufner A, Thomas G (1999): Ribosomal S6 kinase signaling and the control of translation. Exp Cell Res 253, 100-109

Ebert AD, Liang P, Wu JC (2012): Induced pluripotent stem cells as a disease modeling and drug screening platform. J Cardiovasc Pharmacol $\underline{60}$, 408-416

Egan D, Kim J, Shaw RJ, Guan KL (2011): The autophagy initiating kinase ULK1 is regulated via opposing phosphorylation by AMPK and mTOR. Autophagy $\underline{7}$, 643-644

Egan DF, Chun MG, Vamos M, Zou H, Rong J, Miller CJ, Lou HJ, Raveendra-Panickar D, Yang CC, Sheffler DJ, et al. (2015): Small molecule inhibition of the autophagy kinase ULK1 and identification of ULK1 substrates. Mol Cell 푸, 285-297 
Egan DF, Shackelford DB, Mihaylova MM, Gelino S, Kohnz RA, Mair W, Vasquez DS, Joshi A, Gwinn DM, Taylor R, et al. (2011): Phosphorylation of ULK1 (hATG1) by AMPactivated protein kinase connects energy sensing to mitophagy. Science $\underline{331}$, 456-461

Eggert C, Chari A, Laggerbauer B, Fischer U (2006): Spinal muscular atrophy: the RNP connection. Trends Mol Med $\underline{12}, 113-121$

Erlich S, Alexandrovich A, Shohami E, Pinkas-Kramarski R (2007): Rapamycin is a neuroprotective treatment for traumatic brain injury. Neurobiol Dis $\underline{26}$, 86-93

Fawcett JW (2020): The struggle to make CNS axons regenerate: why has it been so difficult? Neurochem Res $\underline{45}, 144-158$

Finkel RS, Mercuri E, Darras BT, Connolly AM, Kuntz NL, Kirschner J, Chiriboga CA, Saito K, Servais L, Tizzano E, et al. (2017): Nusinersen versus sham control in infantile-onset spinal muscular atrophy. N Engl J Med $\underline{377}$, 1723-1732

Fischer LR, Culver DG, Tennant P, Davis AA, Wang M, Castellano-Sanchez A, Khan J, Polak MA, Glass JD (2004): Amyotrophic lateral sclerosis is a distal axonopathy: evidence in mice and man. Exp Neurol 185, 232-240

Gan W, Zhang C, Siu KY, Satoh A, Tanner JA, Yu S (2017): ULK1 phosphorylates Sec23A and mediates autophagy-induced inhibition of ER-to-Golgi traffic. BMC Cell Biol 18, 22

Ganley IG, Lam du H, Wang J, Ding X, Chen S, Jiang X (2009): ULK1.ATG13.FIP200 complex mediates mTOR signaling and is essential for autophagy. J Biol Chem 284, 12297 12305

Gerdts J, Brace EJ, Sasaki Y, DiAntonio A, Milbrandt J (2015): SARM1 activation triggers axon degeneration locally via $\mathrm{NAD}(+)$ destruction. Science $\underline{348}, 453-457$

Gilley J, Orsomando G, Nascimento-Ferreira I, Coleman MP (2015): Absence of SARM1 rescues development and survival of NMNAT2-deficient axons. Cell Rep $\underline{10}$, 1974-1981

Haenseler W, Sansom SN, Buchrieser J, Newey SE, Moore CS, Nicholls FJ, Chintawar S, Schnell C, Antel JP, Allen ND, et al. (2017): A highly efficient human pluripotent stem cell microglia model displays a neuronal-co-culture-specific expression profile and inflammatory response. Stem Cell Reports $\underline{8}, 1727-1742$

Han Z, Chen F, Ge X, Tan J, Lei P, Zhang J (2014): miR-21 alleviated apoptosis of cortical neurons through promoting PTEN-Akt signaling pathway in vitro after experimental traumatic brain injury. Brain Res 1582, 12-20 
He M, Ding Y, Chu C, Tang J, Xiao Q, Luo ZG (2016): Autophagy induction stabilizes microtubules and promotes axon regeneration after spinal cord injury. Proc Natl Acad Sci U S A $\underline{113}, 11324-11329$

Heiman M, Schaefer A, Gong S, Peterson JD, Day M, Ramsey KE, Suarez-Farinas M, Schwarz C, Stephan DA, Surmeier DJ, et al. (2008): A translational profiling approach for the molecular characterization of CNS cell types. Cell 135, 738-748

Hill CS, Coleman MP, Menon DK (2016): Traumatic axonal injury: mechanisms and translational opportunities. Trends Neurosci $\underline{39}, 311-324$

Honda S, Arakawa S, Nishida Y, Yamaguchi H, Ishii E, Shimizu S (2014): Ulk1-mediated Atg5-independent macroautophagy mediates elimination of mitochondria from embryonic reticulocytes. Nat Commun $\underline{5}, 4004$

Hosokawa N, Hara T, Kaizuka T, Kishi C, Takamura A, Miura Y, Iemura S, Natsume T, Takehana K, Yamada N, et al. (2009): Nutrient-dependent mTORC1 association with the ULK1-Atg13-FIP200 complex required for autophagy. Mol Biol Cell 20, 1981-1991

Hou X, Watzlawik JO, Fiesel FC, Springer W (2020): Autophagy in Parkinson's disease. J Mol Biol 432, 2651-2672

Hu Y (2016): Axon injury induced endoplasmic reticulum stress and neurodegeneration. Neural Regen Res 11, 1557-1559

Hu Y, Park KK, Yang L, Wei X, Yang Q, Cho KS, Thielen P, Lee AH, Cartoni R, Glimcher LH, et al. (2012): Differential effects of unfolded protein response pathways on axon injuryinduced death of retinal ganglion cells. Neuron $\underline{73}, 445-452$

Huang DW, Sherman BT, Lempicki RA (2009): Systematic and integrative analysis of large gene lists using DAVID bioinformatics resources. Nat Protoc $\underline{4}, 44-57$

Joo JH, Wang B, Frankel E, Ge L, Xu L, Iyengar R, Li-Harms X, Wright C, Shaw TI, Lindsten T, et al. (2016): The noncanonical role of ULK/ATG1 in ER-to-Golgi trafficking is essential for cellular homeostasis. Mol Cell $\underline{62}$, 491-506

Jung CH, Seo M, Otto NM, Kim DH (2011): ULK1 inhibits the kinase activity of mTORC1 and cell proliferation. Autophagy $\underline{7}, 1212-1221$

Jung CH, Jun CB, Ro SH, Kim YM, Otto NM, Cao J, Kundu M, Kim DH (2009): ULKAtg13-FIP200 complexes mediate mTOR signaling to the autophagy machinery. Mol Biol Cell 20, 1992-2003 
Kerschensteiner M, Schwab ME, Lichtman JW, Misgeld T (2005): In vivo imaging of axonal degeneration and regeneration in the injured spinal cord. Nat Med 11, 572-577

Kim J, Kundu M, Viollet B, Guan KL (2011): AMPK and mTOR regulate autophagy through direct phosphorylation of Ulk1. Nat Cell Biol $\underline{13}, 132-141$

Kleiger G, Mayor T (2014): Perilous journey: a tour of the ubiquitin-proteasome system. Trends Cell Biol 24, 352-359

Klionsky DJ, Abdelmohsen K, Abe A, Abedin MJ, Abeliovich H, Acevedo Arozena A, Adachi H, Adams CM, Adams PD, Adeli K, et al. (2016): Guidelines for the use and interpretation of assays for monitoring autophagy (3rd edition). Autophagy 12, 1-222

Knoferle J, Koch JC, Ostendorf T, Michel U, Planchamp V, Vutova P, Tonges L, Stadelmann C, Bruck W, Bahr M, et al. (2010): Mechanisms of acute axonal degeneration in the optic nerve in vivo. Proc Natl Acad Sci U S A 107, 6064-6069

Ko SH, Apple EC, Liu Z, Chen L (2020): Age-dependent autophagy induction after injury promotes axon regeneration by limiting NOTCH. Autophagy 16, 2052-2068

Koch JC, Tonges L, Michel U, Bahr M, Lingor P (2014a): Viral vector-mediated downregulation of RhoA increases survival and axonal regeneration of retinal ganglion cells. Front Cell Neurosci $\underline{8}, 273$

Koch JC, Knoferle J, Tonges L, Michel U, Bahr M, Lingor P (2011): Imaging of rat optic nerve axons in vivo. Nat Protoc $\underline{6}, 1887-1896$

Koch JC, Tonges L, Barski E, Michel U, Bahr M, Lingor P (2014b): ROCK2 is a major regulator of axonal degeneration, neuronal death and axonal regeneration in the CNS. Cell Death Dis $\underline{5}$, e1225

Koch JC, Tatenhorst L, Roser AE, Saal KA, Tonges L, Lingor P (2018): ROCK inhibition in models of neurodegeneration and its potential for clinical translation. Pharmacol Ther $\underline{189}$, $1-21$

Koch JC, Kuttler J, Maass F, Lengenfeld T, Zielke E, Bahr M, Lingor P (2020): Compassionate use of the ROCK inhibitor fasudil in three patients with amyotrophic lateral sclerosis. Front Neurol 11, 173

Koh JY, Wie MB, Gwag BJ, Sensi SL, Canzoniero LM, Demaro J, Csernansky C, Choi DW (1995): Staurosporine-induced neuronal apoptosis. Exp Neurol 135, 153-159 
Krzywinski M, Schein J, Birol I, Connors J, Gascoyne R, Horsman D, Jones SJ, Marra MA (2009): Circos: an information aesthetic for comparative genomics. Genome Res 19, 16391645

Laemmli UK (1970): Cleavage of structural proteins during the assembly of the head of bacteriophage T4. Nature 227, 680-685

Laplante M, Sabatini DM (2012): mTOR signaling in growth control and disease. Cell $\underline{149}$, 274-293

Lazarus MB, Novotny CJ, Shokat KM (2015): Structure of the human autophagy initiating kinase ULK1 in complex with potent inhibitors. ACS Chem Biol 10, 257-261

Leibinger M, Hilla AM, Andreadaki A, Fischer D (2019): GSK3-CRMP2 signaling mediates axonal regeneration induced by Pten knockout. Commun Biol 2, 318

Leibinger M, Andreadaki A, Golla R, Levin E, Hilla AM, Diekmann H, Fischer D (2017): Boosting CNS axon regeneration by harnessing antagonistic effects of GSK3 activity. Proc Natl Acad Sci U S A 114, E5454-E5463

Li S, Yang L, Selzer ME, Hu Y (2013): Neuronal endoplasmic reticulum stress in axon injury and neurodegeneration. Ann Neurol 74, 768-777

Li WW, Li J, Bao JK (2012): Microautophagy: lesser-known self-eating. Cell Mol Life Sci $\underline{69}$, $1125-1136$

Licatalosi DD, Darnell RB (2006): Splicing regulation in neurologic disease. Neuron $\underline{52}$, $93-$ 101

Lingor P, Koch JC, Tonges L, Bahr M (2012): Axonal degeneration as a therapeutic target in the CNS. Cell Tissue Res 349, 289-311

Lingor P, Teusch N, Schwarz K, Mueller R, Mack H, Bahr M, Mueller BK (2007): Inhibition of Rho kinase (ROCK) increases neurite outgrowth on chondroitin sulphate proteoglycan in vitro and axonal regeneration in the adult optic nerve in vivo. J Neurochem $\underline{103}, 181-189$

Lingor P, Weber M, Camu W, Friede T, Hilgers R, Leha A, Neuwirth C, Gunther R, Benatar M, Kuzma-Kozakiewicz M, et al. (2019): ROCK-ALS: protocol for a randomized, placebocontrolled, double-blind phase IIa trial of safety, tolerability and efficacy of the Rho kinase (ROCK) inhibitor fasudil in amyotrophic lateral sclerosis. Front Neurol $\underline{10}, 293$ 
Liu K, Tedeschi A, Park KK, He Z (2011): Neuronal intrinsic mechanisms of axon regeneration. Annu Rev Neurosci 34, 131-152

Loffler AS, Alers S, Dieterle AM, Keppeler H, Franz-Wachtel M, Kundu M, Campbell DG, Wesselborg S, Alessi DR, Stork B (2011): Ulk1-mediated phosphorylation of AMPK constitutes a negative regulatory feedback loop. Autophagy $\underline{7}$, 696-706

Loh SH, Francescut L, Lingor P, Bahr M, Nicotera P (2008): Identification of new kinase clusters required for neurite outgrowth and retraction by a loss-of-function RNA interference screen. Cell Death Differ 15, 283-298

Martinez-Lopez N, Athonvarangkul D, Mishall P, Sahu S, Singh R (2013): Autophagy proteins regulate ERK phosphorylation. Nat Commun $\underline{4}, 2799$

Matsushita M, Yamamoto R, Mitsui K, Kanazawa H (2009): Altered motor activity of alternative splice variants of the mammalian kinesin-3 protein KIF1B. Traffic 10, 1647-1654

Medana IM, Esiri MM (2003): Axonal damage: a key predictor of outcome in human CNS diseases. Brain 126, 515-530

Meijering E, Jacob M, Sarria JC, Steiner P, Hirling H, Unser M (2004): Design and validation of a tool for neurite tracing and analysis in fluorescence microscopy images. Cytometry A $\underline{58}, 167-176$

Mendoza MC, Er EE, Blenis J (2011): The Ras-ERK and PI3K-mTOR pathways: cross-talk and compensation. Trends Biochem Sci $\underline{36}, 320-328$

Menzies FM, Fleming A, Rubinsztein DC (2015): Compromised autophagy and neurodegenerative diseases. Nat Rev Neurosci 16, 345-357

Menzies FM, Fleming A, Caricasole A, Bento CF, Andrews SP, Ashkenazi A, Fullgrabe J, Jackson A, Jimenez Sanchez M, Karabiyik C, et al. (2017): Autophagy and neurodegeneration: pathogenic mechanisms and therapeutic opportunities. Neuron 93, 1015-1034

Mi H, Huang X, Muruganujan A, Tang H, Mills C, Kang D, Thomas PD (2017): PANTHER version 11: expanded annotation data from Gene Ontology and Reactome pathways, and data analysis tool enhancements. Nucleic Acids Res $\underline{45}$, D183-D189

Michel U, Kallmann B, Rieckmann P, Isbrandt D (2002): UM 9(5)h and UM 9(5)p, human and porcine noncoding transcripts with preferential expression in the cerebellum. RNA $\underline{8}$, $1538-1547$ 
Namikawa K, Honma M, Abe K, Takeda M, Mansur K, Obata T, Miwa A, Okado H, Kiyama $\mathrm{H}$ (2000): Akt/protein kinase B prevents injury-induced motoneuron death and accelerates axonal regeneration. J Neurosci $\underline{20}, 2875-2886$

Niederost BP, Zimmermann DR, Schwab ME, Bandtlow CE (1999): Bovine CNS myelin contains neurite growth-inhibitory activity associated with chondroitin sulfate proteoglycans. J Neurosci 19, 8979-8989

Nik S, Bowman TV (2019): Splicing and neurodegeneration: insights and mechanisms. Wiley Interdiscip Rev RNA 10, e1532

Nikoletopoulou V, Papandreou ME, Tavernarakis N (2015): Autophagy in the physiology and pathology of the central nervous system. Cell Death Differ 22, 398-407

Nishida Y, Arakawa S, Fujitani K, Yamaguchi H, Mizuta T, Kanaseki T, Komatsu M, Otsu K, Tsujimoto Y, Shimizu S (2009): Discovery of Atg5/Atg7-independent alternative macroautophagy. Nature $\underline{461}, 654-658$

Ohri SS, Maddie MA, Zhao Y, Qiu MS, Hetman M, Whittemore SR (2011): Attenuating the endoplasmic reticulum stress response improves functional recovery after spinal cord injury. Glia $\underline{59}, 1489-1502$

Orozco D, Edbauer D (2013): FUS-mediated alternative splicing in the nervous system: consequences for ALS and FTLD. J Mol Med (Berl) 91, 1343-1354

Osterloh JM, Yang J, Rooney TM, Fox AN, Adalbert R, Powell EH, Sheehan AE, Avery MA, Hackett R, Logan MA, et al. (2012): dSarm/Sarm1 is required for activation of an injuryinduced axon death pathway. Science $\underline{337}, 481-484$

Park JW, Vahidi B, Taylor AM, Rhee SW, Jeon NL (2006): Microfluidic culture platform for neuroscience research. Nat Protoc 1 , 2128-2136

Park KK, Liu K, Hu Y, Smith PD, Wang C, Cai B, Xu B, Connolly L, Kramvis I, Sahin M, et al. (2008): Promoting axon regeneration in the adult CNS by modulation of the PTEN/mTOR pathway. Science 322, 963-966

Pellizzoni L, Kataoka N, Charroux B, Dreyfuss G (1998): A novel function for SMN, the spinal muscular atrophy disease gene product, in pre-mRNA splicing. Cell $\underline{95}$, 615-624

Prasad A, Bharathi V, Sivalingam V, Girdhar A, Patel BK (2019): Molecular mechanisms of TDP-43 misfolding and pathology in amyotrophic lateral sclerosis. Front Mol Neurosci 12, 25 
Raff MC, Whitmore AV, Finn JT (2002): Axonal self-destruction and neurodegeneration. Science 296, 868-871

Raivich G, Makwana M (2007): The making of successful axonal regeneration: genes, molecules and signal transduction pathways. Brain Res Rev $\underline{53}$, 287-311

Raivich G, Bohatschek M, Da Costa C, Iwata O, Galiano M, Hristova M, Nateri AS, Makwana M, Riera-Sans L, Wolfer DP, et al. (2004): The AP-1 transcription factor c-Jun is required for efficient axonal regeneration. Neuron $\underline{43}, 57-67$

Ribas VT, Lingor P (2016): Calcium channel inhibition-mediated axonal stabilization improves axonal regeneration after optic nerve crush. Neural Regen Res 11, 1245-1246

Ribas VT, Koch JC, Michel U, Bahr M, Lingor P (2017): Attenuation of axonal degeneration by calcium channel inhibitors improves retinal ganglion cell survival and regeneration after optic nerve crush. Mol Neurobiol 54, 72-86

Ribas VT, Schnepf B, Challagundla M, Koch JC, Bahr M, Lingor P (2015): Early and sustained activation of autophagy in degenerating axons after spinal cord injury. Brain Pathol $\underline{25}, 157-170$

Ribas VT, Vahsen BF, Tatenhorst L, Estrada V, Dambeck V, Almeida RA, Bahr M, Michel U, Koch JC, Muller HW, et al. (2021): AAV-mediated inhibition of ULK1 promotes axonal regeneration in the central nervous system in vitro and in vivo. Cell Death Dis $\underline{12}, 213$

Rigo F, Hua Y, Krainer AR, Bennett CF (2012): Antisense-based therapy for the treatment of spinal muscular atrophy. J Cell Biol 199, 21-25

Rodriguez-Muela N, Germain F, Marino G, Fitze PS, Boya P (2012): Autophagy promotes survival of retinal ganglion cells after optic nerve axotomy in mice. Cell Death Differ 19 , 162-169

Rodriguez-Muela N, Hernandez-Pinto AM, Serrano-Puebla A, Garcia-Ledo L, Latorre SH, de la Rosa EJ, Boya P (2015): Lysosomal membrane permeabilization and autophagy blockade contribute to photoreceptor cell death in a mouse model of retinitis pigmentosa. Cell Death Differ 22, 476-487

Roser AE, Caldi Gomes L, Halder R, Jain G, Maass F, Tonges L, Tatenhorst L, Bahr M, Fischer A, Lingor P (2018): miR-182-5p and miR-183-5p act as GDNF mimics in dopaminergic midbrain neurons. Mol Ther Nucleic Acids 11, 9-22 
Rubinsztein DC, Codogno P, Levine B (2012): Autophagy modulation as a potential therapeutic target for diverse diseases. Nat Rev Drug Discov 11, 709-730

Rubinsztein DC, Bento CF, Deretic V (2015): Therapeutic targeting of autophagy in neurodegenerative and infectious diseases. J Exp Med 212, 979-990

Rudnick ND, Griffey CJ, Guarnieri P, Gerbino V, Wang X, Piersaint JA, Tapia JC, Rich MM, Maniatis T (2017): Distinct roles for motor neuron autophagy early and late in the SOD1(G93A) mouse model of ALS. Proc Natl Acad Sci U S A 114, E8294-E8303

Sahoo PK, Smith DS, Perrone-Bizzozero N, Twiss JL (2018): Axonal mRNA transport and translation at a glance. J Cell Sci 131, jcs196808

Shigeoka T, Jung H, Jung J, Turner-Bridger B, Ohk J, Lin JQ, Amieux PS, Holt CE (2016): Dynamic axonal translation in developing and mature visual circuits. Cell $\underline{166}, 181-192$

Smith PK, Krohn RI, Hermanson GT, Mallia AK, Gartner FH, Provenzano MD, Fujimoto EK, Goeke NM, Olson BJ, Klenk DC (1985): Measurement of protein using bicinchoninic acid. Anal Biochem 150, 76-85

Spaulding EL, Burgess RW (2017): Accumulating evidence for axonal translation in neuronal homeostasis. Front Neurosci 11, 312

Stavoe AKH, Holzbaur ELF (2019): Autophagy in neurons. Annu Rev Cell Dev Biol $\underline{35}$, $477-500$

Szklarczyk D, Morris JH, Cook H, Kuhn M, Wyder S, Simonovic M, Santos A, Doncheva NT, Roth A, Bork P, et al. (2017): The STRING database in 2017: quality-controlled proteinprotein association networks, made broadly accessible. Nucleic Acids Res 4드, D362-D368

Szymanska P, Martin KR, MacKeigan JP, Hlavacek WS, Lipniacki T (2015): Computational analysis of an autophagy/translation switch based on mutual inhibition of MTORC1 and ULK1. PLoS One 10, e0116550

Takahashi K, Yamanaka S (2006): Induction of pluripotent stem cells from mouse embryonic and adult fibroblast cultures by defined factors. Cell $\underline{126}$, 663-676

Taylor AM, Blurton-Jones M, Rhee SW, Cribbs DH, Cotman CW, Jeon NL (2005): A microfluidic culture platform for CNS axonal injury, regeneration and transport. Nat Methods 2, 599-605 
Teyssou E, Takeda T, Lebon V, Boillee S, Doukoure B, Bataillon G, Sazdovitch V, Cazeneuve C, Meininger V, LeGuern E, et al. (2013): Mutations in SQSTM1 encoding p62 in amyotrophic lateral sclerosis: genetics and neuropathology. Acta Neuropathol $\underline{125}, 511-$ 522

The Gene Ontology Consortium (2017): Expansion of the Gene Ontology knowledgebase and resources. Nucleic Acids Res 45, D331-D338

Toda H, Mochizuki H, Flores R, Josowitz R, Krasieva TB, Lamorte VJ, Suzuki E, Gindhart JG, Furukubo-Tokunaga K, Tomoda T (2008): UNC-51/ATG1 kinase regulates axonal transport by mediating motor-cargo assembly. Genes Dev 22, 3292-3307

Tonges L, Planchamp V, Koch JC, Herdegen T, Bahr M, Lingor P (2011): JNK isoforms differentially regulate neurite growth and regeneration in dopaminergic neurons in vitro. $\mathrm{J}$ Mol Neurosci $\underline{45}, 284-293$

Towbin H, Staehelin T, Gordon J (1979): Electrophoretic transfer of proteins from polyacrylamide gels to nitrocellulose sheets - procedure and some applications. Proc Natl Acad Sci U S A $\underline{76}, 4350-4354$

Tremblay F, Brule S, Hee Um S, Li Y, Masuda K, Roden M, Sun XJ, Krebs M, Polakiewicz RD, Thomas G, et al. (2007): Identification of IRS-1 Ser-1101 as a target of S6K1 in nutrientand obesity-induced insulin resistance. Proc Natl Acad Sci U S A 104, 14056-14061

Tzatsos A, Kandror KV (2006): Nutrients suppress phosphatidylinositol 3-kinase/Akt signaling via raptor-dependent mTOR-mediated insulin receptor substrate 1 phosphorylation. Mol Cell Biol 26, 63-76

Vahsen BF, Lingor P (2021): ULK1 as a novel therapeutic target in neurodegeneration. Neural Regen Res 16, 1212-1213

Vahsen BF, Ribas VT, Sundermeyer J, Boecker A, Dambeck V, Lenz C, Shomroni O, Caldi Gomes L, Tatenhorst L, Barski E, et al. (2020): Inhibition of the autophagic protein ULK1 attenuates axonal degeneration in vitro and in vivo, enhances translation, and modulates splicing. Cell Death Differ 27, 2810-2827

Waller A (1850): Experiments on the section of the glossopharyngeal and hypoglossal nerves of the frog, and observations of the alterations produced thereby in the structure of their primitive fibres. Philos Trans R Soc Lond 140, 423-429

Wang B, Iyengar R, Li-Harms X, Joo JH, Wright C, Lavado A, Horner L, Yang M, Guan JL, Frase S, et al. (2018): The autophagy-inducing kinases, ULK1 and ULK2, regulate axon 
guidance in the developing mouse forebrain via a noncanonical pathway. Autophagy $14,796-$ 811

Wang JT, Medress ZA, Barres BA (2012): Axon degeneration: molecular mechanisms of a self-destruction pathway. J Cell Biol 196, 7-18

Wang Y, Song M, Song F (2018): Neuronal autophagy and axon degeneration. Cell Mol Life Sci $\underline{75}, 2389-2406$

Wei HL, Ma SQ, Li CX (2018): Deficiency of unc-51 like kinase 1 (Ulk1) protects against mice traumatic brain injury (TBI) by suppression of p38 and JNK pathway. Biochem Biophys Res Commun $\underline{503}$, 467-473

Yang Y, Coleman M, Zhang L, Zheng X, Yue Z (2013): Autophagy in axonal and dendritic degeneration. Trends Neurosci $\underline{36}, 418-428$

Yang Y, Liu L, Naik I, Braunstein Z, Zhong J, Ren B (2017): Transcription factor C/EBP homologous protein in health and diseases. Front Immunol $\underline{8}, 1612$

Yang Z, Klionsky DJ (2010): Mammalian autophagy: core molecular machinery and signaling regulation. Curr Opin Cell Biol 22, 124-131

Yu CG (2012): Distinct roles for ERK1 and ERK2 in pathophysiology of CNS. Front Biol (Beijing) 7, 267-276

Zachari M, Ganley IG (2017): The mammalian ULK1 complex and autophagy initiation. Essays Biochem $\underline{61}, 585-596$

Zhang J, Gao Z, Yin J, Quon MJ, Ye J (2008): S6K directly phosphorylates IRS-1 on Ser270 to promote insulin resistance in response to TNF-(alpha) signaling through IKK2. J Biol Chem $\underline{283}, 35375-35382$

Zhang JN, Koch JC (2017): Collapsin response mediator protein-2 plays a major protective role in acute axonal degeneration. Neural Regen Res 12, 692-695

Zhang JN, Michel U, Lenz C, Friedel CC, Koster S, d'Hedouville Z, Tonges L, Urlaub H, Bahr M, Lingor P, et al. (2016): Calpain-mediated cleavage of collapsin response mediator protein-2 drives acute axonal degeneration. Sci Rep $\underline{6}, 37050$

Zhang L, Ouyang L, Guo Y, Zhang J, Liu B (2018): UNC-51-like kinase 1: from an autophagic initiator to multifunctional drug target. J Med Chem 61, 6491-6500 
Zhang L, Fu L, Zhang S, Zhang J, Zhao Y, Zheng Y, He G, Yang S, Ouyang L, Liu B (2017): Discovery of a small molecule targeting ULK1-modulated cell death of triple negative breast cancer in vitro and in vivo. Chem Sci $\underline{8}, 2687-2701$

Zhang M, Ergin V, Lin L, Stork C, Chen L, Zheng S (2019): Axonogenesis is coordinated by neuron-specific alternative splicing programming and splicing regulator PTBP2. Neuron 101, 690-706 e610

Zhao C, Takita J, Tanaka Y, Setou M, Nakagawa T, Takeda S, Yang HW, Terada S, Nakata T, Takei Y, et al. (2001): Charcot-Marie-Tooth disease type $2 \mathrm{~A}$ caused by mutation in a microtubule motor KIF1Bbeta. Cell $\underline{105}$, 587-597

Zheng S (2020): Alternative splicing programming of axon formation. Wiley Interdiscip Rev RNA $\underline{11}$, e1585

Zhong L, Li B, Mah CS, Govindasamy L, Agbandje-McKenna M, Cooper M, Herzog RW, Zolotukhin I, Warrington KH, Weigel-Van Aken KA, et al. (2008): Next generation of adeno-associated virus 2 vectors: point mutations in tyrosines lead to high-efficiency transduction at lower doses. Proc Natl Acad Sci U S A $\underline{105}$, 7827-7832

Zhou X, Babu JR, da Silva S, Shu Q, Graef IA, Oliver T, Tomoda T, Tani T, Wooten MW, Wang F (2007): Unc-51-like kinase 1/2-mediated endocytic processes regulate filopodia extension and branching of sensory axons. Proc Natl Acad Sci U S A 104, 5842-5847

Zolotukhin S, Byrne BJ, Mason E, Zolotukhin I, Potter M, Chesnut K, Summerford C, Samulski RJ, Muzyczka N (1999): Recombinant adeno-associated virus purification using novel methods improves infectious titer and yield. Gene Ther $\underline{6}$, 973-985 


\section{Danksagung}

Zuallererst gilt mein besonderer Dank Herrn Prof. Dr. Paul Lingor für den umfangreichen Einblick in die medizinische Forschung, den mir dieses spannende und anspruchsvolle Promotionsprojekt gegeben hat. Herzlichen Dank für die exzellente Betreuung und Unterstützung über die gesamte Länge dieser Doktorarbeit, aber vor allem auch für das Interesse an und die Förderung meiner beruflichen und wissenschaftlichen Zukunft.

Des Weiteren bedanke ich mich bei Herrn Prof. Dr. Mathias Bähr für die Bereitstellung ausgezeichneter Arbeitsbedingungen im Bäbr Lab der Neurologie sowie für die Unterstützung meines Projektes durch ein Promotionsstipendium.

Mein Dank gilt ebenfalls Herrn Prof. Dr. Henning Urlaub und Herrn Dr. Christof Lenz für die freundliche und kompetente Zusammenarbeit im Rahmen der Proteomanalyse sowie Frau Dr. Gabriela Salinas und Herrn Dr. Orr Shomroni für die Durchführung der Transkriptomanalyse. Herzlichen Dank hierbei auch an Herrn Dr. Lucas Caldi Gomes für die geduldige Unterstützung während dieses Teilprojektes.

Furthermore, I am deeply grateful to Prof Dr Vinicius de Toledo Ribas not only for the patient and knowledgeable introduction to lab work and, more broadly, science, but also for the excellent supervision, even from Brazil. Muito obrigado!

Darüber hinaus möchte ich mich bei Frau Elisabeth Barski und Frau Vivian Dambeck für die großartige technische Unterstützung im Labor und die exzellenten Ratschläge bedanken. Besonders herzlichen Dank auch für die unschätzbare Hilfe bei der Revision der CDDPublikation, die ohne Euch und Herrn Dr. Lars Tatenhorst nicht möglich gewesen wäre.

Großer Dank gebührt ebenfalls Frau Dr. Anna-Elisa Roser für die zahlreichen Hilfestellungen und Erklärungen, die ein Medizindoktorand ohne Wissenschaftserfahrung benötigte.

Schließlich möchte ich mich bei allen Mitgliedern der AG Lingor, ganz besonders auch den noch nicht genannten Frau Dr. Eleonora Carboni, Frau Dr. Karina Joppe, Frau Dr. KimAnn-Saal und meinem Mitdoktoranden Herrn Dr. Julius Steenken, für die großartige, konstruktive, aber auch spaßige Arbeitsatmosphäre bedanken. Es war eine tolle Zeit zusammen, auch außerhalb des Labors. 